\title{
COMPENSATION TECHNIQUES IN ACCELERATOR PHYSICS
}

\author{
by \\ Hisham Kamal Sayed \\ B.S. May 2001, Cairo University \\ M.S. December 2007, Old Dominion University
}

A Dissertation Submitted to the Faculty of

Old Dominion University in Partial Fulfillment of the

Requirements for the Degree of

DOCTOR OF PHILOSOPHY

PHYSICS

OLD DOMINION UNIVERSITY

May 2011

Approved by:

Geoffrey Krafft (Director)

Alex Bogacz

Hari Areti

Jean Delayen

Hussein Abdel-Wahab 


\title{
ABSTRACT \\ COMPENSATION TECHNIQUES IN ACCELERATOR PHYSICS
}

\author{
Hisham Kamal Sayed \\ Old Dominion University, 2011 \\ Director: Dr. Geoffrey Krafft
}

Accelerator physics is one of the most diverse multidisciplinary fields of physics, wherein the dynamics of particle beams is studied. It takes more than the understanding of basic electromagnetic interactions to be able to predict the beam dynamics, and to be able to develop new techniques to produce, maintain and deliver high quality beams for different applications. In this work, some basic theory regarding particle beam dynamics in accelerators will be presented. This basic theory, along with applying state of the art techniques in beam dynamics will be used in this dissertation to study and solve accelerator physics problems. Two problems involving compensation are studied in the context of the MEIC (Medium Energy Electron Ion Collider) project at Jefferson Laboratory. Several chromaticity (the energy dependence of the particle tune) compensation methods are evaluated numerically and deployed in a figure eight ring designed for the electrons in the collider. Furthermore, transverse coupling optics have been developed to compensate the coupling introduced by the spin rotators in the MEIC electron ring design. 
(C)Copyright, 2011, by Hisham Kamal Sayed, All Rights Reserved 
I dedicate this thesis to the men and women who lost their lives on January 25, 2011 in Tahrir Square for us to see a free Egypt. 


\section{ACKNOWLEDGMENTS}

I would like to offer my heartily thanks and gratitude to my advisors Alex Bogacz and Geoffrey Krafft, for allowing me the opportunity to work at the forefront of beam physics. They showed continuous patience, encouragement, and support throughout my journey as a graduate student doing my research at Jefferson Laboratory, I am greatly indebted to them. I owe a debt of gratitude to Andrew Hutton for his precious mentoring and invaluable scientific discussions which helped me in formulating my vision for this thesis. I thank all CASA group members including S. Derbenev, P. Chevtsov, Y. Roblin, R. Li, M. Tiefenback, B. Terzic,Y. Zhang, S. Ahmed, and B. Yunn. They introduced me to accelerator physics, they gave advice when needed, and helped me during my research while working on the Electron ion collider at Jefferson laboratory.

During my graduate school I was invited to participate in the commissioning of the ERL photoinjector at Cornell University. I would like to thank Ivan Bazarov for giving me such great opportunity.

I thank all my friends and colleagues at Old Dominion University, especially my all time friend M. Hegazy who always was there for me; with him I enjoyed great discussions on physics, politics, and poems. I am grateful to my friend Islam Bedir who was an inspiration for me. I thank Serkan Golge for helping me getting this dissertation in format, and $\mathrm{R}$. Bodenstein for reading through my dissertation and giving valuable suggestions.

I want to express special debt of gratitude to my parents Kamal Sayed and Safaa Abdulrazzaq; without their love and support this project would not have been possible. Special thanks are due to my wife Nermeen Khalil for her understanding and love during the past few years. Her support and encouragement was invaluable for completion of this dissertation. I also thank my daughter Sumayah and my son Hamzah; they filled my life with joy and love.

Praise and thanks are due to Allah, the Exalted, for his guidance, provisions, mercy, and blessing on my family and myself. I thank Allah for making it possible for me to complete this dissertation. 


\section{TABLE OF CONTENTS}

List of Tables ......................... . . ix

List of Figures . . . . . . . . . . . . . . . . . . . . . . xiii

Chapter

I An electron ion collider for Jefferson Lab . . . . . . . . . . . . . . . . 1

I.1 Introduction . . . . . . . . . . . . . . . . . . . . 1

I.1.1 Luminosity in lepton hadron colliders . . . . . . . . . . . . . . 3

I.1.2 Equations of linear motion and terminology of linear optics . . 5

I.2 CEBAF as the full energy injector for MEIC . . . . . . . . . . . . . . 9

I.3 The lepton hadron collider HERA . . . . . . . . . . . . . . . . . 12

I.3.1 Arc lattice and interaction regions . . . . . . . . . . . . . 12

I.3.2 Polarized lepton beam . . . . . . . . . . . . . . . . . . 12

I.4 Figure-8 ring layout and linear optics design . . . . . . . . . . . . . . . . 16

I.4.1 Arcs . . . . . . . . . . . . . . . . . . . . . 18

I.4.2 Dispersion in the straight sections . . . . . . . . . . . . . 25

I.4.3 Interaction region . . . . . . . . . . . . . . . . . . . . . . 29

I.4.4 Spin rotators ...................... 37

I.5 Summary . . . . . . . . . . . . . . . . . . . . . 37

II Chromaticity and chromaticity correction . . . . . . . . . . . . 38

II.1 Introduction . . . . . . . . . . . . . . . . . . . . . . . . . . . . . . . . . . . . . .

II.2 Hill's equations . . . . . . . . . . . . . . . . . . . . . . . . . . . . . . . . . . . . . . . . .

II.3 Tune chromaticity in collider rings . . . . . . . . . . . . . . . . . . . . 41

II.3.1 First order chromaticity . . . . . . . . . . . . . . . . . . . . 42

II.3.2 Second order tune chromaticity . . . . . . . . . . . . . . . . 43

II.4 Chromaticity in case of a straight beamline . . . . . . . . . . . . . . . 44

II.4.1 Compensating chromaticity in a straight interaction region . . 45

II.5 Beta chromaticity in the final focus . . . . . . . . . . . . . . . . . . . . . . . . 47

II.6 Chromaticity correction schemes . . . . . . . . . . . . . . . . . . . . . 49

II.6.1 Global chromatic correction . . . . . . . . . . . . . . . . 49

II.6.2 Local chromatic correction . . . . . . . . . . . . . . . . . 50

II.6.3 Phase advance adjustment between the two $\beta$-insertions . . . 51

II.7 Touschek lifetime . . . . . . . . . . . . . . . . . . 52

II.7.1 Computing Touschek lifetime . . . . . . . . . . . . . . . 53

II.7.2 Touschek lifetime in case of constant momentum acceptance . 54

II.7.3 Touschek lifetime in case of variable dynamic transverse momentum acceptance . . . . . . . . . . . 54

II.8 Beam-beam tune shift . . . . . . . . . . . . . . . . . . . . . . 58

II.9 Radiation power . . . . . . . . . . . . . . . . . . . . 59

II.10 Summary . . . . . . . . . . . . . . . . . . . . . . . . . . . . . . . . . . . . . . . 60

III Chromaticity correction for MEIC . . . . . . . . . . . . . . . . 61 
III.1 Introduction . . . . . . . . . . . . . . . . . . . . . . . . . . . 61

III.2 Natural chromaticity from the arc sections . . . . . . . . . . . . . . . 61

III.2.1 Arc lattice with $135^{\circ}$ phase advance cells . . . . . . . . . . . . 61

III.2.2 Arc lattice with $90^{\circ}$ phase advance cells . . . . . . . . . . . . . 65

III.3 Analysis of chromaticity of the final focusing quadrupoles . . . . . . . 68

III.4 Correcting chromaticity for the MEIC . . . . . . . . . . . . . . . . . 68

III.4.1 Anti-symmetric dispersion around the interaction point . . . . 69

III.4.2 Symmetric dispersion around the interaction point . . . . . . . 76

III.5 Results and discussion . . . . . . . . . . . . . . . . . . . 84

IV Polarized electron beams in figure-8 rings . . . . . . . . . . . . . . . . . . 85

IV.1 Requirements for polarized beams for MEIC . . . . . . . . . . . . . . . . 85

IV.2 Introduction to polarized beams . . . . . . . . . . . . . . . 85

IV.2.1 Thomas-BMT equation . . . . . . . . . . . . . . . . . 86

IV.2.2 Radiative Polarization (Sokolov-Ternov Effect) . . . . . . . . . 87

IV.3 Coupled motion in a solenoid field . . . . . . . . . . . . . . 87

IV.4 Generalizing the Courant-Snyder parametrization to two dimensional coupled linear motion . . . . . . . . . . . . . . . . . . . 9 90

IV.5 Polarization around the figure-8 ring . . . . . . . . . . . . . . . . . 91

IV.6 Universal spin rotators . . . . . . . . . . . . . . . . . . . . . . . . . . 92

IV.7 Coupling compensation insert . . . . . . . . . . . . . . . . . . . . . . . . . . . . 93

IV.8 Integration of spin rotators to MEIC . . . . . . . . . . . . . . . . . 96

IV.8.1 Stability study . . . . . . . . . . . . . . . . . . . . . 101

IV.9 Results and discussion . . . . . . . . . . . . . . . . . . . . . . . . . . . . . . . . . . . . . . . . . . . .

V Electron Ion Collider ELIC . . . . . . . . . . . . . . . . 102

V.1 Lattice design . . . . . . . . . . . . . . . . . . . . . . . . . . . 102

V.1.1 Arc sections . . . . . . . . . . . . . . . 103

V.1.2 Straight section . . . . . . . . . . . . . . . . . . 104

V.1.3 The interaction region . . . . . . . . . . . . . . 107

V.2 Chromaticity correction . . . . . . . . . . . . . . 110

V.2.1 Local chromaticity correction . . . . . . . . . . . . . . 110

V.2.2 Global chromaticity correction . . . . . . . . . . . . . . . 110

V.2.3 Touschek lifetime . . . . . . . . . . . . . . . . 113

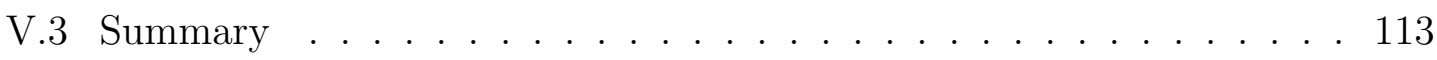

VI Conclusions . . . . . . . . . . . . . . . . . . . 115

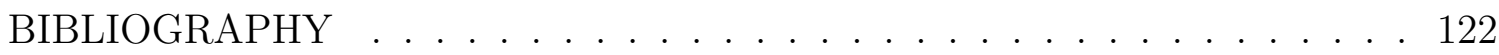

\section{APPENDICES}

A Appendix: Beam dynamics codes ................... 123

A.1 ELEGANT . . . . . . . . . . . . . . . . . . 123

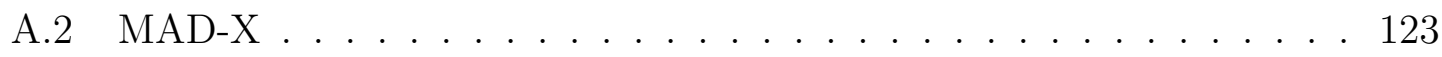

A.3 Touschek lifetime code . . . . . . . . . . . . . . . . . . 125

B Appendix: MEIC lattices in MAD-X format . . . . . . . . . . . . . . . . 129

B.1 MEIC $90^{\circ}$ lattices in MAD-X format . . . . . . . . . . . . . . . 129 
B.2 MEIC $135^{\circ}$ lattices in MAD-X format . . . . . . . . . . . . . . . 136

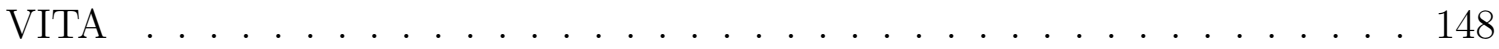




\section{LIST OF TABLES}

Page

1 CEBAF beam parameters currently and in the future $12 \mathrm{GeV}$ upgrade 11

2 HERA design parameters . . . . . . . . . . . . . . . . . . 13

3 Design parameters for MEIC (Medium energy electron ion collider at JLAB) . . . . . . . . . . . . . . . . . . . . . . 16

4 Magnet parameters of a MEIC $135^{\circ}$ cell. $L, \theta$, and $K$ are magnet length, bending angle, and quadrupole focusing strength respectively. 19

5 Parameters of a MEIC $135^{\circ}$ lattice . . . . . . . . . . . . . . . 20

6 Magnet parameters of a MEIC $90^{\circ}$ cell. . . . . . . . . . . . . . . . 22

7 Parameters of a MEIC $90^{\circ}$ lattice. . . . . . . . . . . . . . . . . . 23

8 Magnet lattice parameters of a MEIC interaction region. . . . . . . . 32

9 Magnet lattice parameters of a MEIC IR. . . . . . . . . . . . . . . . . 35

10 Estimates of the first order chromaticity from the MEIC final focus quadrupoles with non-vanishing dispersion derivative at the interaction point. . . . . . . . . . . . . . . . 69

11 Estimates of the first order chromaticity from the MEIC final focus quadrupoles with vanishing dispersion derivative at the interaction point. . . . . . . . . . . . . . . . . 69

12 Magnets of the interaction region matching section. . . . . . . . . . 76

13 Magnets of the chromaticity correction block. . . . . . . . . . . . 78

14 Local correcting sextupole families. . . . . . . . . . . . . . . . . . . . 79

15 Magnet parameters for universal spin rotator (USR) at different beam energies. $\phi_{1,2}$ and DBL are the solenoid spin rotation angle and integral field strength respectively. $\theta_{1,2}$ are the dipole spin rotation angles. 94

16 Magnets of the coupling compensation insert. . . . . . . . . . . . 96

17 Design parameters for the MEIC and the ELIC. . . . . . . . . . . . . 102

18 Magnet parameters of an ELIC arc cell. . . . . . . . . . . . . . . . 103

19 Parameters of an ELIC lattice. . . . . . . . . . . . . . . . . . . 105

20 Magnet parameters of the ELIC interaction region. . . . . . . . . . . 108

21 Local correcting sextupole families. . . . . . . . . . . . . . . . . . 110

22 Global correcting sextupole families. . . . . . . . . . . . . . . . . 112 


\section{LIST OF FIGURES}

Page

1 Schematic view of the MEIC/ELIC layout at Jefferson Lab. . . . . . 2

2 Detailed layout of the MEIC with injection from current Jefferson Lab CEBAF. . . . . . . . . . . . . . . . 3

3 Schematic of CEBAF showing different components of the current 6 $\mathrm{GeV}$ machine plus the $12 \mathrm{GeV}$ upgrade accelerating section and new experimental Hall D. . . . . . . . . . . . . . . . . . . . . . . . . 10

4 The CEBAF $50 \mathrm{~cm}$ effective length 5-cell cavity. It is a $1497 \mathrm{MHz} \mathrm{RF}$ cavity with a $5 \mathrm{MV} / \mathrm{m}$ energy gain. . . . . . . . . . . . . . 11

5 The $12 \mathrm{GeV}$ upgrade CEBAF single 7-cell cavity. It has $70 \mathrm{~cm}$ effective length with $1497 \mathrm{MHz}$ RF with $19 \mathrm{MV} / \mathrm{m}$ energy gain. . . . . . . . . 11

6 Schematic view of the HERA accelerator complex, showing both electron (red) and proton (blue) rings, injection chain, and four experimental halls. . . . . . . . . . . . . . . . . . . . . 14

7 Schematic view of HERA proton accelerator complex. . . . . . . . . . 15

8 Equilibrium spin polarization vector around the HERA electron ring. The required electron polarization is achieved in 35 minutes. Spin rotators are used to rotate vertical polarization in the arc to longitudinal polarization at interaction regions and vice versa. . . . . . . . . . 15

9 Twiss function of MEIC $135^{\circ}$ arc cell . . . . . . . . . . . . . . . . . 20

10 Twiss functions of MEIC $135^{\circ}$ figure-8 ring. . . . . . . . . . . . . . . 21

11 Twiss functions of MEIC $90^{\circ}$ arc cell . . . . . . . . . . . . . . . 23

12 MEIC $90^{\circ}$ Twiss functions . . . . . . . . . . . . . . . . . . . . . . . . . . . . . . . 24

13 Footprint of MEIC $90^{\circ}$ lattice . . . . . . . . . . . . . . . . . 24

14 Dispersion in straight section I (non - vanishing dispersion derivative at IR) . . . . . . . . . . . . . . . . . . . . . . . 26

15 Dispersion in straight section I (dispersion and its derivative vanish at IR) . . . . . . . . . . . . . . . . . . . . 27

16 Schematic of dispersion suppressor; $\left(T, T_{1}, T_{2}\right)$ are dipoles with bending angles $\left(\theta, \theta_{1}, \theta_{2}\right)$ respectively $\ldots \ldots \ldots . \ldots . \ldots 28$

17 Dispersion suppressed in straight section II . . . . . . . . . . . . . . . 28

18 Schematic of MEIC central detector showing two conical sections on each side to track particles that end up in the endcaps, a radial part includes a complete set of detectors for tracking, identification, and calorimetry. . . . . . . . . . . . . . . . . .

19 Schematic of MEIC detector IR layout corresponding to $50 \mathrm{mrad}$ crossing angle ...................... . . . 31

20 Twiss parameters through the interaction region with non vanishing dispersion derivative. . . . . . . . . . . . . . . . . . . 32

21 Twiss parameters through two mirror symmetric IRs at the same straight with non vanishing dispersion derivative. . . . . . . . . 
22 Beam size at the interaction region, the horizontal beam size at IP is $8.3 \times 10^{-5} \mathrm{~m}$ and $1.7 \times 10^{-5} \mathrm{~m}$ in the vertical. The maximum amplitude is $5.38 \mathrm{~mm}$ in horizontal dimension and $2.89 \mathrm{~mm}$ in vertical dimension. 34

23 Twiss parameters through interaction region with vanishing dispersion derivative at the interaction point. . . . . . . . . . . . . . . 35

24 Beam size at the interaction region, the horizontal beam size at interaction point is $2.8 \times 10^{-5} \mathrm{~m}$ and $1.26 \times 10^{-5} \mathrm{~m}$ in the vertical. The maximum amplitude is $3.8 \mathrm{~mm}$ in horizontal dimension and $2.08 \mathrm{~mm}$ in vertical dimension. . . . . . . . . . . . . . . . . . 36

25 Energy-dependent increase in spot size . . . . . . . . . . . . . . . . 49

26 Touschek lifetime for the $9 \mathrm{GeV}$ MEIC $135^{\circ}$ lattice. . . . . . . . . . . 55

27 Touschek lifetime for the $9 \mathrm{GeV}$ MEIC $90^{\circ}$ lattice. . . . . . . . . . . . 55

28 Touschek lifetime for the $9 \mathrm{GeV}$ MEIC $135^{\circ}$ lattice as a function of the minimum size of the beam pipe half width. . . . . . . . . . . . 56

29 Touschek lifetime for the $9 \mathrm{GeV}$ MEIC $90^{\circ}$ lattice as a function of the minimum size of the beam pipe half width. . . . . . . . . . . . . 57

30 Tune space for the electron beam. High order resonant lines are shown in various colors. The line for which the ratio is equal to the golden mean is shown in green. Small red and blue dots represent the tunes of the 400 random orbits from the electron and proton beams, respectively. Large red and blue dots represent design tunes for electron and proton beams. . . . . . . . . . . . . . . . . .

31 Synchrotron radiation power per unit beam current as a function of bending radius at three different electron energies. The strong dependence of radiation power on the electron energy causes substantial limitations on the maximum achievable energy in circular accelerators. $\quad 59$

32 MEIC arc cells with sextupole magnets (smallest boxes). Sextupoles are placed at locations with good aspect ratios between $\beta_{x}$ and $\beta_{y}$. . .

33 Tune variation with momentum deviation for the MEIC figure- 8 ring without the interaction region. Momentum acceptance is $\pm 0.8 \%$. . .

34 MEIC arc cells with sextupole magnets (smallest boxes). Sextupoles are placed at locations with good aspect ratio between $\beta_{x}$ and $\beta_{y}$. . . 66

35 Tune variation with momentum deviation for MEIC figure- 8 ring without the interaction region. The momentum acceptance is $\pm 2.0 \%$. . . 67

36 Twiss function near the IR with a non-vanishing dispersion derivative at the IP. . . . . . . . . . . . . . . . . . . . 70

37 MEIC Montague chromatic functions at the IP after correction with local sextupoles. Sextupoles are placed at locations with a good aspect ratio between $\beta_{x}$ and $\beta_{y}$. . . . . . . . . . . . . . . .

38 MEIC Montague chromatic functions in the two interaction regions after correction with local sextupoles. . . . . . . . . . . . . . . 72

39 MEIC Montague chromatic functions in the figure- 8 ring after sextupole correction. . . . . . . . . . . . . . . . 
MEIC tune variation with momentum deviation before and after correction. Momentum acceptance is $\pm 0.3 \%$. . . . . . . . . . . . . . . 74

41 Twiss functions at the chromaticity correction block. . . . . . . . . . 77

42 MEIC Montague chromatic functions at the IP after correction with the local sextupoles. . . . . . . . . . . . . . . . . . . . . 80

43 MEIC Montague chromatic functions after correction with local and global sextupoles. Sextupoles were placed at locations with good aspect ratios between $\beta_{x}$ and $\beta_{y}$ and reasonable dispersion. . . . . . . . 81

44 MEIC Montague chromatic functions in one arc cell after correction. . 82

45 MEIC tune variation with momentum deviation after correction. Momentum acceptance is $\pm 0.3 \%$. . . . . . . . . . . . . . . . . . . . 82

46 Dynamic aperture study for MEIC. Red stars represent initial amplitudes while blue dots represent surviving particles. . . . . . . . . . 83

47 Spin vector for the electron beam polarization around the figure- 8 ring 92

48 Layout of the Universal Spin Rotator (USR) (solenoids are black, quadrupoles are blue and dipoles are green) . . . . . . . . . . . . 93

$49 \beta$-functions across the symmetric insertion between two identical solenoids. . . . . . . . . . . . . . . . . . 97

50 Dispersion function across the USR. One can see that although the electron ring is a totally horizontal, the horizontal dispersion passing through the solenoid is coupled and vertical dispersion is generated. Vertical dispersion is suppressed by the end of the second half of the solenoid due to rotation introduced by the coupling compensation insert. 97

51 Twiss functions through the MEIC figure- 8 ring with the integrated and matched USR. . . . . . . . . . . . . . . . . . . . . . . . . . . . . 98

52 Twiss functions through the USR. The USR was matched at the end of the arc section and to the start of the straight section. . . . . . . . 99

53 Dispersion through the MEIC figure-8 ring with the integrated and matched USR. Note that the vertical dispersion vanishes at the end of the USR. . . . . . . . . . . . . . . . . . . 100

54 Error analysis for MEIC coupling compensation insert. . . . . . . . . 101

55 Twiss function of ELIC arc cell . . . . . . . . . . . . . . . . . . . . . 104

56 Suppressing dispersion in the beginning of straight section II. . . . . . 105

57 Twiss functions of ELIC figure-8 ring. . . . . . . . . . . . . . 106

58 Twiss functions in the interaction region. The dispersion function vanishes at the IP, while the derivative of dispersion does not vanish at IP. . . . . . . . . . . . . . . . . . . . . . . . . . . . 107

59 Beam size at interaction region, the horizontal beam size at IP is $5.2 \times 10^{-5} \mathrm{~m}$ and $2.3 \times 10^{-6} \mathrm{~m}$ in the vertical, the maximum amplitude is $20 \mathrm{~mm}$ in horizontal and $29 \mathrm{~mm}$ in vertical. . . . . . . . . . . . . . 108

60 Twiss functions of ELIC with two interaction regions on one straight section. . . . . . . . . . . . . . . . 109

61 Montague chromatic functions across ELIC IR after local chromatic correction using a set of 4 sextupole families. . . . . . . . . . . . . . . 111 
62 Tune variation with momentum deviation for ELIC after correction. The momentum acceptance was limited to $3-6 \times 10^{-5}$ due to large initial chromaticity from the interaction region final focusing quadrupoles and very small $\beta^{*}$. . . . . . . . . . . . . . . . . . . . . . . . . . . . . 112

63 Touschek lifetime for ELIC. . . . . . . . . . . . . . . 113 


\section{CHAPTER I}

\section{AN ELECTRON ION COLLIDER FOR JEFFERSON LAB}

\section{I.1 INTRODUCTION}

The proposed Medium Energy Electron Ion Collider (MEIC) [1] at Thomas Jefferson National Accelerator Facility (JLAB) will be primarily composed of two figure-8 rings, intersecting at up to four collision points, with a proton energy of 30-60 GeV (15-30 GeV/A for ions) and an electron (positron) energy of 3-11 GeV. The design luminosity approaches $10^{34} \mathrm{~cm}^{-2} \mathrm{sec}^{-1}$ and MEIC operations are compatible with simultaneous operation of the $12 \mathrm{GeV}$ CEBAF for fixed-target experiments [2]. A requirement driven by nuclear science program is high polarization for both beams, up to $80 \%$. The design is being carried out with an upgrade to higher energies in mind called ELIC (ELectron Ion Collider), where high luminosity and high polarization continue to be the design drivers.

A possible electron ring design will be presented in this thesis, that will be based on a number of conservative MEIC design assumptions [3]. The first assumption is that the maximum peak magnetic field of any superconducting dipole is less than 6 Tesla for the ion ring. This limit puts a constraint on the minimum ring size. Noting that any tunnel built has to fit both electron and ion rings to minimize the cost, the electron ring size is chosen based on this constraint as well. The second assumption is that the maximum synchrotron radiation power density does not exceed $20 \mathrm{~kW} / \mathrm{m}$, consistent with high current ring experience [4]. The final assumption is that the maximum allowed $\beta$-function in the final focus region has to be less than $2.5 \mathrm{~km}$ in order for the chromaticity not to degrade beam stability.

As seen in Fig. (1) [1], the existing CEBAF accelerator with a polarized electron beam will be used as a full energy injector into a 3-11 GeV electron storage ring. A positron source is envisioned as a future addition to the CEBAF injector for generating positrons that can be accelerated in CEBAF, accumulated and polarized in the electron storage ring, and collided with ions with luminosity similar to the electron/ion collisions [5].

The MEIC facility is designed to produce a variety of polarized ion species: $\mathrm{p}, \mathrm{d}$,

This dissertation follows the style of Physical Review Special Topics-Accelerators and Beams as journal model. 


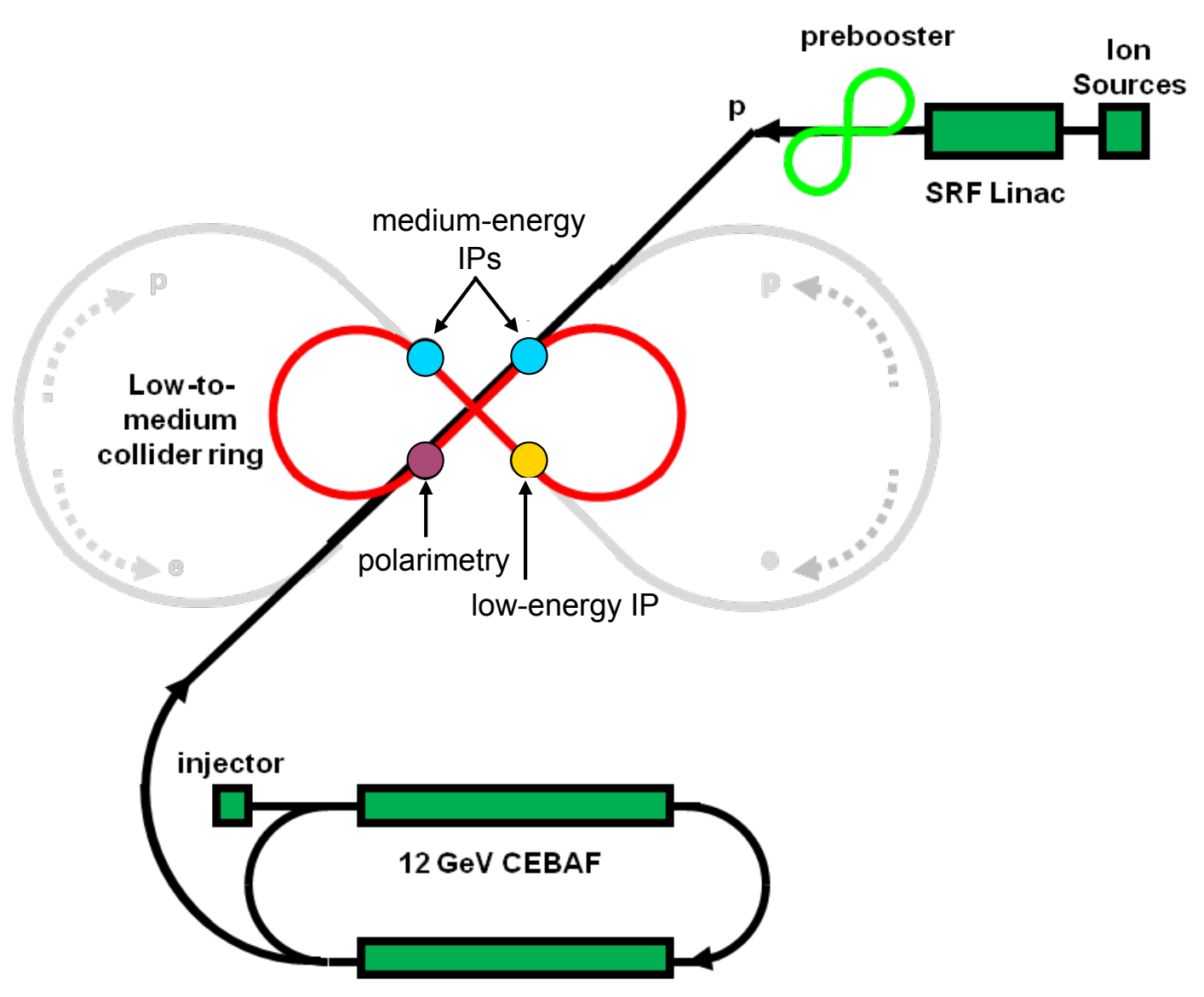

FIG. 1: Schematic view of the MEIC/ELIC layout at Jefferson Lab.

$\mathrm{He}^{3}$ and $\mathrm{Li}$, and unpolarized light ion species. To attain the required ion beams, a new ion facility must be constructed at Jefferson Laboratory, a major component of which is the $60 \mathrm{GeV}$ collider ring located in the same tunnel and above the electron storage ring. A critical component of the ion complex is an energy recovery linac (ERL)based continuous electron cooling facility, anticipated to provide low emittance and very short ion bunches simultaneously.

The MEIC design accommodates an upgrade to higher ion beam energies up to $250 \mathrm{GeV}$, with luminosity close to $10^{35} \mathrm{~cm}^{-2} \mathrm{sec}^{-1}$ [6]. Fig. (1) shows a representation of the upgrade project in gray color [1]. 


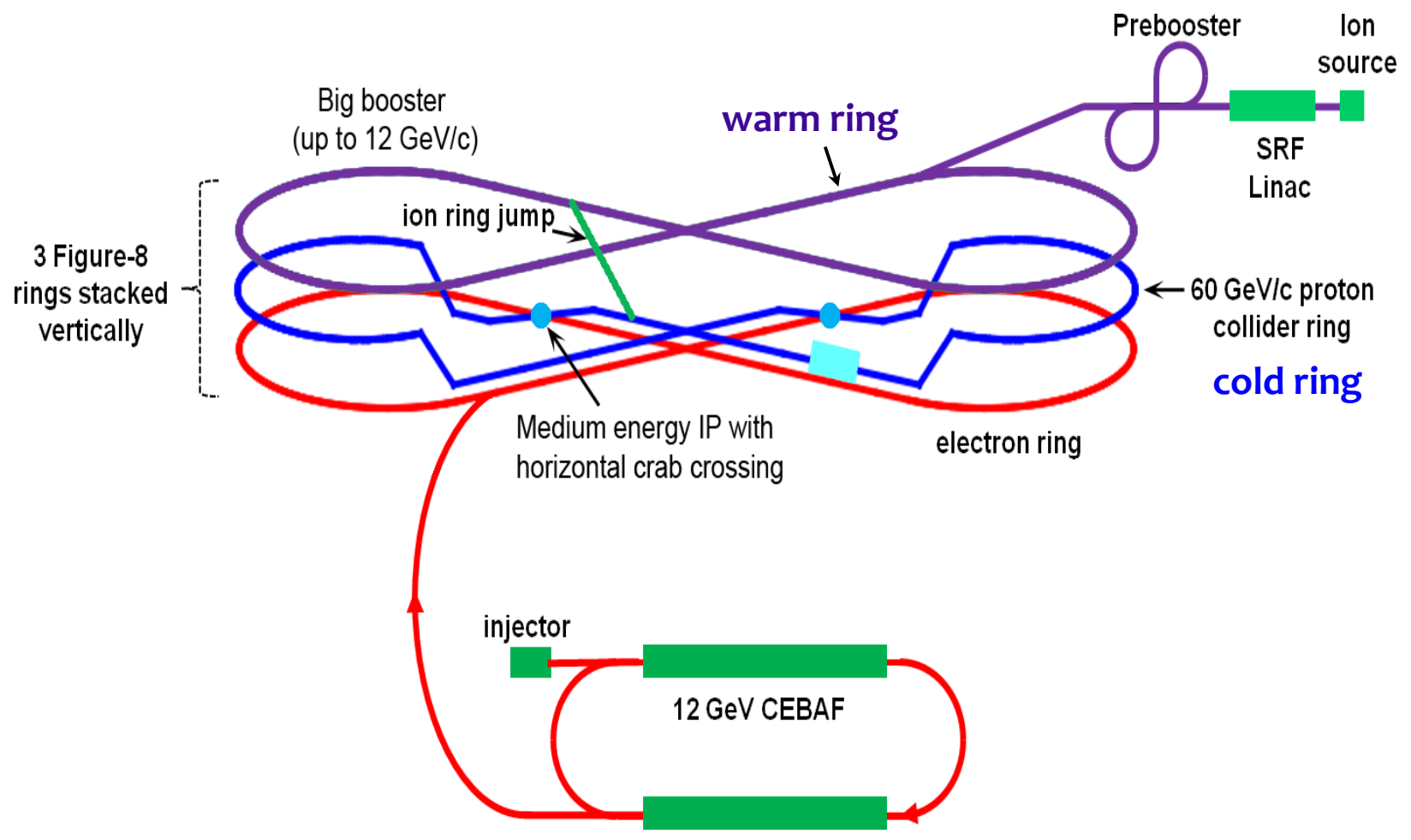

FIG. 2: Detailed layout of the MEIC with injection from current Jefferson Lab CEBAF.

\section{I.1.1 Luminosity in lepton hadron colliders}

The accelerator luminosity, representing the collision rate, is an important figure of merit for any collider. The collider luminosity is defined as the ratio of the event rate of a chosen interaction at a beam crossing to the effective beam cross-section area at that interaction point. The luminosity depends on both the beam parameters and the accelerator lattice.

In the MEIC case of a lepton hadron collider in a double ring machine, the collider may be assumed to operate with head-on collisions between both beam bunches. The general luminosity formula for such machines is given by: 


$$
L=\frac{N_{e} N_{p} N_{B} f_{r e v}}{2 \pi \sqrt{\sigma_{x p}^{2}+\sigma_{x e}^{2}} \sqrt{\sigma_{y p}^{2}+\sigma_{y e}^{2}}},
$$

where $N_{p}\left(N_{e}\right)$ is the number of protons (electrons) per bunch, $N_{B}$ is the number of bunches, $f_{r e v}$ revolution frequency, and $\sigma_{p}\left(\sigma_{e}\right)$ is proton (electron) rms beam size at the interaction point (IP).

The optimum conditions for such a machine are given as follows [7]

1. The hadron and lepton beam cross sections have to match to limit the nonlinearity of the beam-beam interaction. Therefore $\sigma_{x p} \simeq \sigma_{x e}$ and $\sigma_{y p} \simeq \sigma_{y e}$.

2. The total beam current of the lepton beam is limited by the synchrotron radiation maximum power mentioned previously.

3. $N_{p}$ is limited by space charge effects in the ion injector chain.

4. The minimum beam size at the interaction point is limited by the maximum proton beam size within the final focus quadrupoles of the interaction region due to a maximum achievable focusing strength possible at an appropriate magnet aperture size, and to the ability to compensate for the chromaticity generated. This limitation is quantified by a maximum value of beta function $\beta_{\text {max }}$, to be defined in subsequent text.

These conditions lead to a lepton hadron collider luminosity formula which has a major dependence on hadron beam parameters through the number of hadrons per bunch and bunch transverse size, and a dependence on the electron beam current as

$$
L=\frac{I_{e} N_{p} \gamma_{p}}{4 \pi e \sqrt{\beta_{x p}^{*} \beta_{y p}^{*}} \sqrt{\epsilon_{x N} \epsilon_{y N}}} .
$$

In the MEIC case where the hadron beam is assumed to be round after cooling $\left(\epsilon_{x N} \approx \epsilon_{y N}\right)$, the final luminosity equation is given by

$$
L=\frac{I_{e} N_{p} \gamma_{p}}{4 \pi e \sqrt{\beta_{x p}^{*} \beta_{y p}^{*}} \epsilon_{p N}} .
$$

From Eq. (3) we can see that in order to increase the collider luminosity two main parameters are left to manipulate.

1. Minimize $\beta_{p}^{*}$. This will introduce chromaticity in the collider ring, which in turn affects the stability and the lifetime of the beam. 
2. Minimize $\epsilon_{p N}$. This parameter will be limited by the ion beam cooling.

The significance of the first parameter in the ultimate MEIC luminosity is the main motivation to find an appropriate scheme for correcting chromaticity of both beams, and forms the principal justification of this thesis. For MEIC, obtaining small $\epsilon_{p N}$ is being addressed by developing novel cooling techniques, which are beyond the scope of this thesis.

\section{I.1.2 Equations of linear motion and terminology of linear optics}

Within the linear approximation, including effects of a pure quadrupole field and ignoring higher perturbations, the equations of motion for a particle orbit in a periodic system will take the following form

$$
u^{\prime \prime}+k_{u}(s) u=\frac{1}{\rho} \frac{\Delta p}{p},
$$

where $u$ refers to either of the transverse coordinates $(x, y)$, and $\rho$ is the radius of curvature. The focusing strength $k_{u}(s)$ is periodic with a period of length $L$ where $L$ is the is circumference of the circular accelerator lattice,

$$
k_{u}(s)=k_{u}(s+L)
$$

The derivation of these equations of motion from a Hamiltonian will be discussed in more detail in Chapter II.2. The solution to the homogenous part of Eq. (4) $(\Delta p / p=0)$ with a periodic focusing strength may be formulated in terms of Floquet's theorem. This theorem enables us to write the solution of this homogeneous equation of motion in terms of an action $I$ and an initial phase $\psi$ as

$$
u(s)=\sqrt{2 I \beta(s)} \cos (\mu(s)+\psi),
$$

where $\beta(s)$ is the periodic betatron function, and $\mu(s)$ is the characteristic phase coefficient defined by

$$
\mu(s)=\int_{s_{0}}^{s} \frac{1}{\beta(s)} d s .
$$

In these formulas and the rest of the formulas in this section, the implicit $u$ subscript has been dropped in order to simplify the formulas. 
Twiss parameters If $\alpha$ is defined in terms of the first derivative $u^{\prime}(s)$ by

$$
u^{\prime}(s)=\sqrt{\frac{2 I}{\beta(s)}}(\alpha(s) \cos (\mu(s)+\psi)+\sin (\mu(s)+\psi)),
$$

the derivative may be written as

$$
u^{\prime}(s)=\sqrt{2 I \gamma(s)} \cos (\mu(s)+\psi)
$$

with

$$
\alpha(s)=-\frac{\beta^{\prime}(s)}{2}
$$

and

$$
\gamma(s)=\frac{1+\alpha^{2}(s)}{\beta(s)} .
$$

The periodic linear motion around the accelerator can be described by these so called Twiss functions $(\alpha, \beta, \gamma)$. The action $I$ is an invariant of motion, and is usually referred to as Courant-Snyder invariant, where

$$
\gamma(s) z^{2}(s)+2 \alpha(s) z(s) z^{\prime}(s)+\beta(s) z^{\prime 2}(s)=2 I .
$$

The area of the phase space ellipse the orbit is on is called the emittance $(\epsilon)$. It is also an invariant of the motion, and $\epsilon=2 I$. For the electron beam in MEIC the particle distribution in equilibrium is a Gaussian distribution in phase space, with the distribution function

$$
\Psi\left(x, x^{\prime} ; s\right) d x d x^{\prime}=\frac{1}{2 \pi \epsilon_{x}} e^{-\left(\gamma_{x}(s) x^{2}+2 \alpha_{x}(s) x x^{\prime}+\beta_{x}(s) x^{\prime 2}\right) / 2 \epsilon_{x}} d x d x^{\prime},
$$

and likewise for the $y$-direction. By integrating the distribution function, one can calculate the average amplitude, average position squared, and average slope squared of the distribution and obtain:

$$
\begin{aligned}
& \epsilon_{r m s}=\sqrt{<z^{2}><z^{\prime 2}>-<z z^{\prime}>^{2}} \\
& <z^{2}>=\beta \epsilon_{r m s} \\
& <z z^{\prime}>=-\alpha \epsilon_{r m s} \\
& <z^{\prime 2}>=\gamma \epsilon_{r m s} .
\end{aligned}
$$

By Liouville's theorem the phase space ellipse area is conserved in linear motion. In the case of uncoupled motion the area of the phase space ellipse in each of the transverse dimensions is invariant as well. 


\section{Dispersion and Momentum Compaction Factor}

As a straight forward example of the solution of the inhomogeneous equation of motion for off-momentum particle, take a bending magnet with a homogenous magnetic field and with no gradient magnetic field. The equation of motion is

$$
x^{\prime \prime}+\frac{1}{\rho^{2}} x=\frac{1}{\rho} \frac{\Delta p}{p} .
$$

Defining a special trajectory $\eta(s)$ which has $\Delta p / p=1$, Eq. (15) becomes:

$$
\eta^{\prime \prime}(s)+\frac{1}{\rho^{2}} \eta(s)=\frac{1}{\rho} .
$$

The function $\eta(s)$ is called the dispersion function. Eq. (16), which is an inhomogeneous differential equation, has the following solution

$$
\begin{gathered}
\eta(s)=\eta_{0} \cos \frac{s}{\rho}+\eta_{0}^{\prime} \sin \frac{s}{\rho}+\rho\left(1-\cos \frac{s}{\rho}\right) \\
\eta^{\prime}(s)=-\frac{\eta_{0}}{\rho} \sin \frac{s}{\rho}+\eta_{0}^{\prime} \cos \frac{s}{\rho}+\sin \frac{s}{\rho},
\end{gathered}
$$

where $\eta(0)=\eta_{0}$ and $\eta^{\prime}(0)=\eta_{0}^{\prime}$. Now the trajectory of a particle with a momentum deviation $\Delta p / p$ is given by

$$
x_{d}=x(s)+\eta(s) \frac{\Delta p}{p}
$$

The transformation matrix of the dispersion function is a $3 \times 3$-matrix and is given by the following:

$$
\left(\begin{array}{c}
\eta(s) \\
\eta^{\prime}(s) \\
1
\end{array}\right)=\left(\begin{array}{ccc}
\cos \frac{s}{\rho} & \rho \sin \frac{s}{\rho} & \rho\left(1-\cos \frac{s}{\rho}\right) \\
-\frac{1}{\rho} \sin \frac{s}{\rho} & \cos \frac{s}{\rho} & \sin \frac{s}{\rho} \\
0 & 0 & 1
\end{array}\right) \cdot\left(\begin{array}{c}
\eta\left(s_{0}\right) \\
\eta^{\prime}\left(s_{0}\right) \\
1
\end{array}\right)
$$

Similar $3 \times 3$ dispersion transport matrices may be defined for other magnet elements (quadrupoles, drifts, etc.), and are generally calculated by standard computer codes and used to predict the optics properties of accelerator layouts.

\section{Momentum Compaction Factor}

The momentum compaction factor is defined as the ratio of the relative change in path length $\Delta L / L$ to the relative momentum deviation from the reference particle momentum, $\Delta p / p$, and is given by

$$
\alpha_{c}=\frac{\Delta L / L}{\Delta p / p} .
$$


Only bending magnets contribute to the momentum compaction. For a full storage ring of total length $L, \alpha_{c}$ is calculated by

$$
\alpha_{c}=\frac{1}{L} \oint \frac{\eta(s)}{\rho(s)} d s .
$$

Betatron phase advance The total betatron phase advance is defined as the number of betatron oscillations the particle accumulates as it travels around the ring. It is given by

$$
\mu(s)=\int_{s_{0}}^{s} \frac{1}{\beta(s)} d s,
$$

where $s_{0}$ is the beginning point, and $s$ is the end point for the phase advance calculation. In circular accelerators the machine tune is defined as the number of betatron oscillations the particle accumulates as it travels once around the ring. It is given by

$$
\xi_{x, y}=\frac{1}{4 \pi} \int_{s_{0}}^{s_{0}+L} \frac{1}{\beta_{x, y}(s)} d s
$$

where $L$ is the total path length around the accelerator. It does not depend on the starting point $s_{0}$ as the $\beta$ function is periodic. 


\section{I.2 CEBAF AS THE FULL ENERGY INJECTOR FOR MEIC}

The Continuous Electron Beam Accelerator Facility (CEBAF) at JLAB will serve as a full energy injector for MEIC. Currently running at $6 \mathrm{GeV}$ beam energy with a fixed target electron scattering experiments that take place at three user halls (Hall A, Hall $\mathrm{B}$, and Hall C), CEBAF uses superconducting technology (SRF) for electron beam acceleration. CEBAF is presently being upgraded to double the maximum beam energy to $12 \mathrm{GeV}[8]$. During the upgrade a new set of highly improved cryomodules will be added to double the beam energy. In addition, the upgrade includes a new user hall (Hall D). The electron beam polarization reaches $80 \%$ during present operations. Table 1 shows a summary of the CEBAF beam parameters plus the parameters of the $12 \mathrm{GeV}$ upgrade.

The CEBAF layout is shown in Fig. (3). The basic components of CEBAF are

Gun and Injector: Electron beam is continuously emitted from an electron gun with three diode lasers working at $499 \mathrm{MHz}$ repetition rate each with a phase difference of $120^{\circ}$ to deliver beam simultaneously to the three user halls. The bunch length is controlled by choppers and the laser pulse length, followed by a set of bunchers downstream of the gun, accelerating cavities, and an injector chicane.

North and South Linacs: Each linac accelerates the electron beam up to $600 \mathrm{MeV}$ (i.e., for first pass from $65 \mathrm{MeV}$ to $665 \mathrm{MeV}$ ).

Arcs: The first arc section bends the beam by $180^{\circ}$ degrees to be injected in the second linac for further acceleration. Then the beam is transferred by the second arc to the following linac and so on. For every path the beam goes through a different set of bending magnets in a vertically stacked structure of fours paths or five paths.

Accelerating cavities: Super-conducting radio frequency (SRF) 5-cell cavities running at frequency of $1497 \mathrm{MHz}$, with up to $10 \mathrm{MV} / \mathrm{m}$ energy gain, see Fig. (4). Cavities have an elliptical shape and are made of Niobium. In the $12 \mathrm{GeV}$ upgrade some of the existing cavities will be replaced with the new 7-cell (C100) type cavities. The 7-cell cavity has a low power loss with $1497 \mathrm{MHz} \mathrm{RF}$ and reaches $19 \mathrm{MV} / \mathrm{m}$ energy gain, see Fig. (5). 
User Halls (A, B and C) beamlines: After 5 passes of acceleration the total beam energy reaches $6 \mathrm{GeV}$. A set of normal conducting RF separators are used to deliver the beam to user halls, each working at frequency of $499 \mathrm{MHz}$.

The 12 GeV CEBAF will be able to support simultaneous operation of Jefferson Laboratory's fixed target experiments plus the operation of the MEIC figure- 8 collider ring. As the MEIC physics program requires a range of energies from 3-11 GeV, the beam extraction from CEBAF may be accomplished by ejecting the beam after the number of passes corresponding to MEIC's required energy. CEBAF's polarized electron source will provide the required $80 \%$ polarization for MEIC's electron beam.

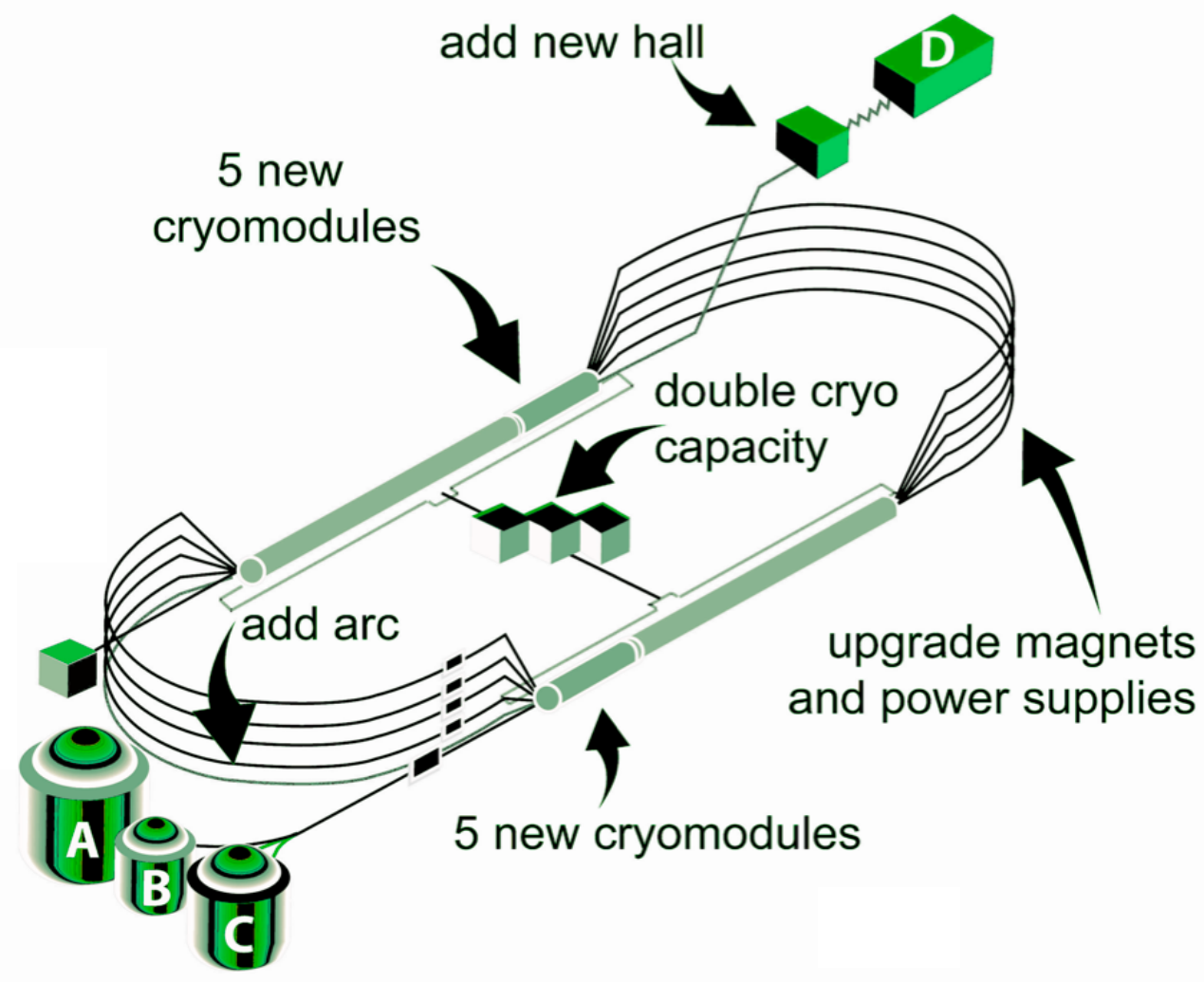

FIG. 3: Schematic of CEBAF showing different components of the current $6 \mathrm{GeV}$ machine plus the $12 \mathrm{GeV}$ upgrade accelerating section and new experimental Hall D. 
TABLE 1: CEBAF beam parameters currently and in the future $12 \mathrm{GeV}$ upgrade

\begin{tabular}{lccc}
\hline \hline Quantity & Unit & $\mathbf{6}$ GeV CEBAF & 12 GeV CEBAF \\
\hline Max. beam energy & $\mathrm{GeV}$ & 6 & 12 \\
Injected beam energy & $\mathrm{GeV}$ & 0.065 & 0.126 \\
Transverse $r m s$ emittance $\epsilon_{x}\left(\epsilon_{y}\right)$ & $\mathrm{nm}$ & $1.0(1.0)$ & $7.0(1.0)$ \\
Energy Spread & & $1 \times 10^{-4}$ & $2 \times 10^{-4}$ \\
Duty factor & $\%$ & $\mathrm{CW}$ & $\mathrm{CW}$ \\
Polarization & 85 & 85 \\
Max. summed current to Halls A\&C & $\mu \mathrm{A}$ & 180 & 85 \\
Max. summed current to Halls B (D) & $\mu \mathrm{A}$ & 0.2 & $5(5)$ \\
\hline \hline
\end{tabular}

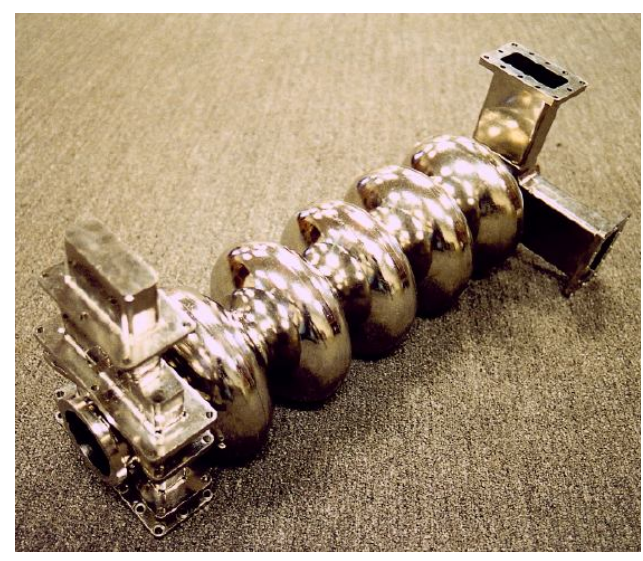

FIG. 4: The CEBAF $50 \mathrm{~cm}$ effective length 5-cell cavity. It is a $1497 \mathrm{MHz}$ RF cavity with a $5 \mathrm{MV} / \mathrm{m}$ energy gain.



FIG. 5: The $12 \mathrm{GeV}$ upgrade CEBAF single 7-cell cavity. It has $70 \mathrm{~cm}$ effective length with $1497 \mathrm{MHz}$ RF with $19 \mathrm{MV} / \mathrm{m}$ energy gain. 


\section{I.3 THE LEPTON HADRON COLLIDER HERA}

The first collisions between high energy electrons and protons were registered at HERA in 1991 [9]. HERA consisted of two $6.4 \mathrm{~km}$ long storage rings, designed to store $820 \mathrm{GeV}$ protons and $30 \mathrm{GeV}$ electrons and to collide the two counter rotating beams head-on in four interaction regions spaced equidistant around its $6.3-\mathrm{km}$ circumference. The proton ring was built from 5 Tesla superconducting magnets while the electron ring was built from normal conducting magnets. The beams collided at four collision points where detectors named H1, ZEUS, HERMES, and HERA-B were located. The interaction regions (IR) were upgraded for a high luminosity of $5 \times 10^{31} \mathrm{~cm}^{-2} \mathrm{sec}^{-1}$ in 2001 [10]. Fig. (6) illustrates the layout of the HERA accelerator complex.

The HERA tunnel consisted of four $90^{\circ}$ arcs joined by $360 \mathrm{~m}$ straight sections. The proton beam energy was limited to $820 \mathrm{GeV}$ by the 4.68 Tesla bending field of the dipole magnets. The electron energy was limited to $30 \mathrm{GeV}$ by the energy loss due to the synchrotron radiation. Electron energy loss was compensated by a RF system providing a total voltage of $200 \mathrm{MV}$, this RF system consisted of warm 500 $\mathrm{MHz}$ cavities augmented by 16 four-cell, $500 \mathrm{MHz}$ superconducting cavities.

Protons generated at the proton source were accelerated to $750 \mathrm{keV}$ by the radio frequency quadrupole (RFQ), to $50 \mathrm{MeV}$ by the Linac III, to $7.5 \mathrm{GeV}$ in DESY III, to $40 \mathrm{GeV}$ in PETRA, and finally to $920 \mathrm{GeV}$ at HERA, see Fig. (7).

\section{I.3.1 Arc lattice and interaction regions}

HERA's proton ring consisted of a FODO (focusing - non-focusing - defocusing non-focusing) cell lattice. Each FODO cell had a $90^{\circ}$ betatron phase advance, and was $47 \mathrm{~m}$ long. Each cell contained four bending magnets with length $8.83 \mathrm{~m}$. The electron ring consisted of a $60^{\circ}$ FODO lattice, each cell was $23.5 \mathrm{~m}$ long. At the end of each arc dispersion was suppressed. The $\beta$ functions in the electron straight section were kept small. The magnetic field errors in HERA caused a small dynamic aperture of only $3.8 \mathrm{rms}$ beam sizes.

\section{I.3.2 Polarized lepton beam}

The HERA lepton beam was spin polarized by the Sokolov-Ternov effect [11]. A set of spin rotators provided longitudinally polarized leptons for collisions with protons. 
The spin rotator was composed of a set of interleaved vertical and horizontal dipole magnets which were used to rotate the vertical (with respect to the direction of electron motion) spin of the electron to longitudinal at the interaction points (IPs), and back to vertical after the IP. The spin rotators had no focusing elements resulting in large $\beta$ functions within the spin rotator regions. Fig. (8) shows schematic of HERA rings with the spin rotator locations indicated.

TABLE 2: HERA design parameters

\begin{tabular}{lccc}
\hline \hline Quantity & Unit & Electron & Proton \\
\hline Beam energy & $\mathrm{GeV}$ & 27.5 & $920 \mathrm{GeV}$ \\
Revolution Frequency & $\mathrm{sec}^{-1}$ & 47317 & 47317 \\
Particles per bunch & $10^{10}$ & 3.65 & 10 \\
Beam average current & $\mathrm{mA}$ & 58 & 163 \\
Polarization time & $\mathrm{min}$ & 35 & - \\
Polarization & $\%$ & $30-45$ & - \\
Energy Spread & & $10^{-3}$ & $10^{-4}$ \\
RMS bunch length & $\mathrm{cm}$ & 0.85 & 19 \\
Transverse beam emittance $\epsilon_{x} / \epsilon_{y}$ & $10^{-9} \mathrm{~m}$ & $41 / 5.1$ & $8 / 3.4$ \\
Beam size at IP $\sigma_{x} / \sigma_{y}$ & $\mathrm{~mm}$ & $0.286 / 0.06$ & $0.28 / 0.058$ \\
Distance from IP to first FF quadrupole & $\mathrm{m}$ & 5.5 & 5.5 \\
Luminosity per IP & $\mathrm{cm}^{-2} \mathrm{sec}$ & $3-5 \times 10^{31}$ & $3-5 \times 10^{31}$ \\
Bending magnet length & $\mathrm{m}$ & 9.185 & 8.824 \\
Bending radius in the arcs & $\mathrm{m}$ & 608.1 & 584 \\
Energy loss per turn & $\mathrm{MeV}$ & 125 & $6 \times 10^{-6}$ \\
Synchrotron radiation power & $\mathrm{MW}$ & 7.2 & $10^{-6}$ \\
Tune Shift $\Delta \xi_{x} / \Delta \xi_{y}$ & & $0.02 / 0.02$ & $10^{-3} / 5 \times 10^{-4}$ \\
Horizontal (vertical) betatron tune & & $47.2(48.35)$ & $31.3(32.3)$ \\
Synchrotron tune & & 0.07 & $1.6 \times 10^{-3}$ \\
RF frequency & $\mathrm{MHz}$ & 500 & $208 / 52$ \\
\hline \hline
\end{tabular}




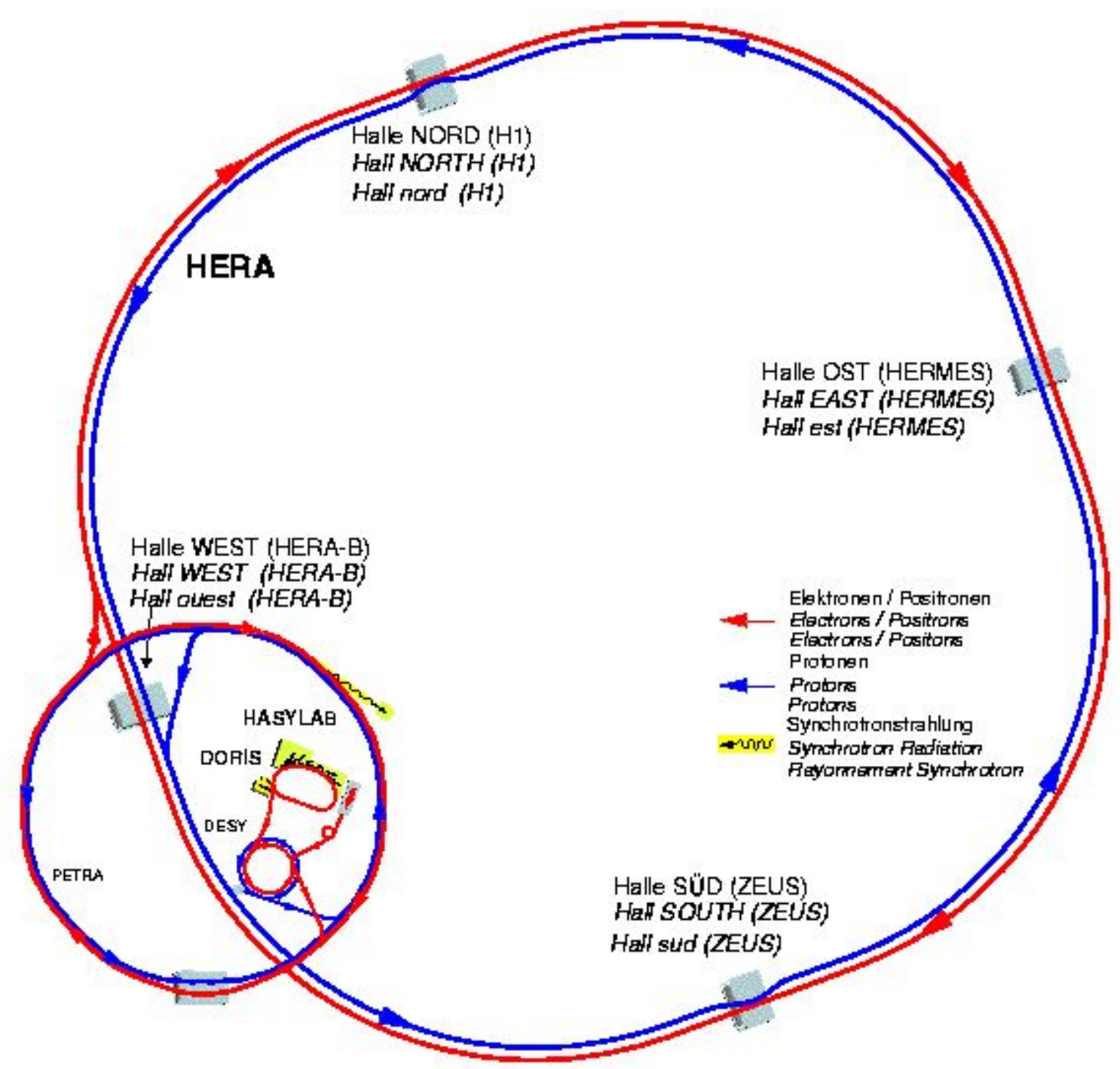

FIG. 6: Schematic view of the HERA accelerator complex, showing both electron (red) and proton (blue) rings, injection chain, and four experimental halls. 


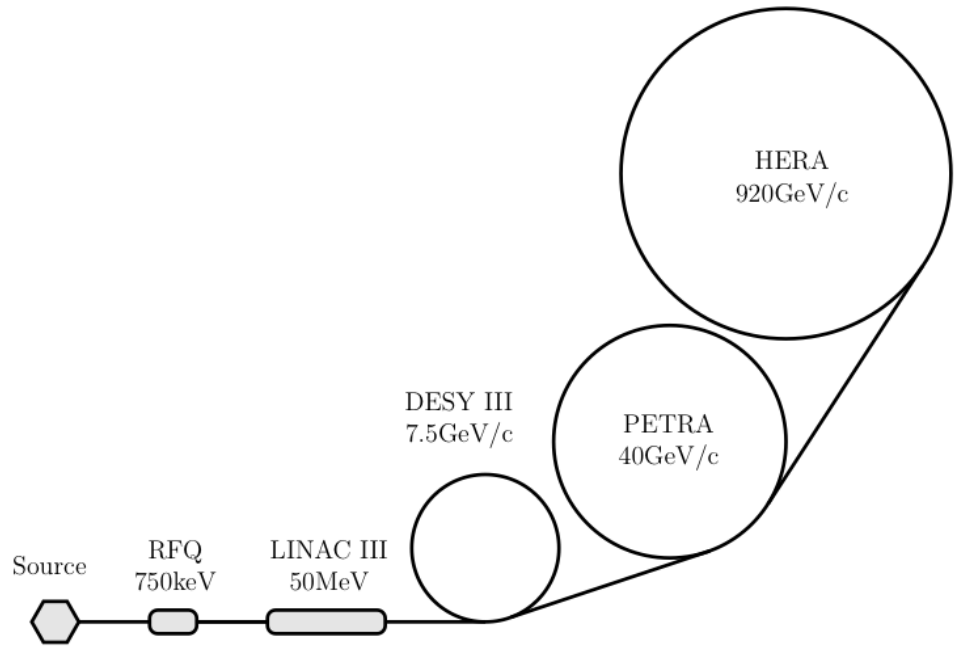

FIG. 7: Schematic view of HERA proton accelerator complex.

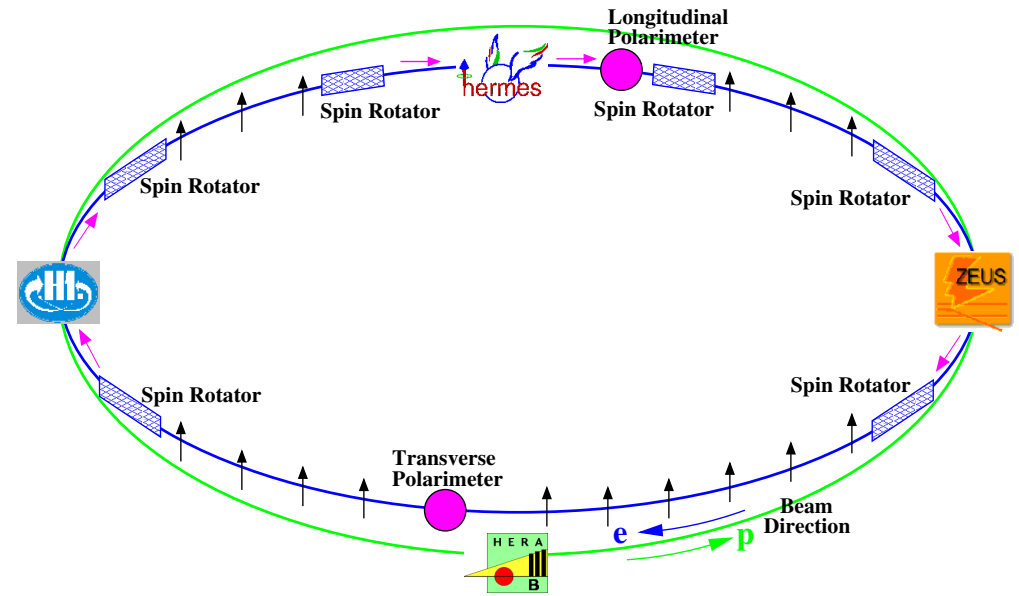

FIG. 8: Equilibrium spin polarization vector around the HERA electron ring. The required electron polarization is achieved in 35 minutes. Spin rotators are used to rotate vertical polarization in the arc to longitudinal polarization at interaction regions and vice versa. 


\section{I.4 FIGURE-8 RING LAYOUT AND LINEAR OPTICS DESIGN}

The rest of this thesis will be addressing the MEIC electron ring only. In this section the requirements for the MEIC electron ring will be presented. Then a linear lattice design which fulfills these requirements will be laid out.

The magnet lattice of MEIC has to satisfy the following requirements:

1 The lattice must have a small enough equilibrium emittance in the electron ring.

The emittance depends on the choice of the arc cell design.

2 The lattice must accommodate a range of energies $(3-11 \mathrm{GeV})$.

3 The lattice must include a number of symmetric low- $\beta$ insertions points (IP) with very small $\beta^{*}$.

4 The dynamic aperture and beam lifetime must be sufficient.

5 The lattice must maintain the beam polarization above $80 \%$ with longitudinal polarization at the IP by adopting a series of spin rotators and spin tuning solenoids.

TABLE 3: Design parameters for MEIC (Medium energy electron ion collider at JLAB).

\begin{tabular}{lccc}
\hline \hline Quantity & Unit & Proton & Electron \\
\hline Beam energy & $\mathrm{GeV}$ & 60 & $3-11 \mathrm{GeV}$ \\
Collision Frequency & $\mathrm{GHz}$ & 0.75 & 0.75 \\
Particles per bunch & $10^{10}$ & 0.416 & 2.5 \\
Beam current & $\mathrm{A}$ & 0.5 & 3 \\
Polarization & $\%$ & 70 & 80 \\
RMS bunch length & $\mathrm{mm}$ & 10 & 7.5 \\
Normalized horizontal emittance & $\mu \mathrm{m} \mathrm{rad}$ & 0.35 & 54 \\
Normalized vertical emittance & $\mu \mathrm{m} \mathrm{rad}$ & 0.07 & 11 \\
Horizontal $\beta^{*}$ & $\mathrm{~cm}$ & 10 & 10 \\
Vertical $\beta^{*}$ & $\mathrm{~cm}$ & 2 & 2 \\
Distance from IP to first FF quadrupole & $\mathrm{m}$ & 7 & 3.5 \\
Luminosity per IP & $\mathrm{cm}^{-2} \mathrm{sec}^{-1}$ & $10^{32}-10^{34}$ & $10^{32}-10^{34}$ \\
\hline \hline
\end{tabular}


The full parameter list for MEIC is given in Table (3) [1]. The general layout is based on a figure- 8 shaped vertically stacked rings as in Fig. (2), two of them for the ion beam, while the third is for the electron (or positron) beam. Polarized ion beam is produced by the ion source and initially accelerated by a superconducting radio frequency (SRF) linac, followed by a figure-8 prebooster while maintaining the required polarization of the beam. The ion beam is next injected from the prebooster into the first figure- 8 ring (warm ring) for acceleration, and then injected into the 60 GeV collider ring (cold ring). Figure- 8 shaped ion rings have a number of positive effects on beam's polarization [12] which can be summarized in the following points:

$\diamond$ This choice is a simple solution to preserve full ion polarization during acceleration by avoiding spin resonances during acceleration.

$\diamond$ Figure-8 rings have energy independence of spin tune.

$\diamond$ Because $(g-2)$ is small for deuterons; a figure-8 ring seems the only practical way to accelerate deuterons and to arrange for longitudinal spin polarization at the interaction point.

These attractive features are compelling enough to adopt an ion ring figure- 8 footprint in MEIC, and the electron ring must be shaped in the same way. Aside from providing the overall ring dimensions for MEIC and the figure- 8 configuration of the electron ring, the ion complex will not be discussed further in this thesis.

For the electrons, polarized electrons from the CEBAF machine are injected at full energy directly into the figure- 8 electron ring. The electron and ion beams are brought into collision by vertically bending the ion beam. This choice was made to limit the effect of synchrotron radiation had the electron beam been bent vertically. The $12 \mathrm{GeV}$ CEBAF, having polarized beam, meets the electron injector requirements for the MEIC. In Chapter IV we will give a detailed discussion of the electron beam polarization and spin rotation.

The main components of MEIC's electron ring magnetic lattice can be summarized in the following items:

1 Two identical arcs with opposite bending directions.

2 The first long straight section contains. 
- A dispersion matching section.

- Two spin rotators.

- An interaction region matching section.

- Two symmetric interaction regions (or one interaction region).

3 The second long straight section contains.

- Two spin rotators.

- Dispersion adjusting section.

\section{I.4.1 Arcs}

The design of the arc cells has to meet two main requirements. The first requirement is that low equilibrium emittance must be achieved, while the second is to make a specific choice of phase advance per cell, related to the natural chromaticity generated by cell's quadrupoles. In addition to the mentioned constraints we have to take into account that sextupole magnets will be added to the arc cells for chromaticity corrections. This can be done by tailoring the dispersion function close to quadrupole magnets where it is preferred to place sextupole magnets.

During the design and optimization process we have considered two different cell designs; the first one with $135^{\circ}$ betatron phase advance and the second with $90^{\circ}$ phase advance. In this section the basic characteristics of each of the designs will be presented. In Chapter III.2, a comparison study regarding their chromaticity and dynamic aperture will be discussed.

\section{Lattice With $135^{\circ}$ Phase Advance Cells}

One FODO cell of the magnetic lattice is shown in Fig. (9), along with their $\beta$ function and dispersion. Each cell of the arc is composed of two quadrupole magnets with alternating focusing and defocusing strength and two horizontally bending dipoles. The magnet parameters are given in Table (4). The basic structure of the two arc regions is the same except that the dipoles bend the beam in opposite directions to form a figure- 8 . The two straight sections are composed of the same FODO cells, plus a matching section, and the interaction region.

The total path length of the figure- 8 ring is $660 \mathrm{~m}$. The horizontal equilibrium emittance and other lattice parameters are collected in Table (5). The choice of a 
TABLE 4: Magnet parameters of a MEIC $135^{\circ}$ cell. $L, \theta$, and $K$ are magnet length, bending angle, and quadrupole focusing strength respectively.

\begin{tabular}{lccc}
\hline \hline Magnet & $L[\mathrm{~m}]$ & $\theta[\mathrm{rad}]$ & $K\left[\mathrm{~m}^{-2}\right]$ \\
\hline QUADRUPOLE & 0.5 & 0.0 & 1.583 \\
QUADRUPOLE & 0.5 & 0.0 & -1.581 \\
DIPOLE ARC I & 1.5 & -0.06159 & 0 \\
DIPOLE ARC II & 1.5 & 0.06159 & 0 \\
\hline \hline
\end{tabular}

FODO lattice with $135^{\circ}$ phase advance per cell was considered to obtain the required low horizontal equilibrium emittance and at the same time be able to construct a $-I$ transformation required for correcting chromatic aberrations. The dispersion function is well tailored to add chromatic correction sextupoles at places with well separated horizontal and vertical $\beta$ functions [13]. Twiss functions $(\beta, \eta)$ for the whole figure- 8 ring are shown in Fig. (10). 
TABLE 5: Parameters of a MEIC $135^{\circ}$ lattice

\begin{tabular}{lc}
\hline \hline Quantity & Value \\
\hline Beam energy $E$ & $9 \mathrm{GeV}$ \\
Particles/bunch $N$ & $3.1 \times 10^{10}$ \\
Bunch length $\sigma_{s}$ & $5 \times 10^{-3} \mathrm{~m}$ \\
Energy loss/turn $U_{0}$ & $33.45 \mathrm{MeV}$ \\
Horizontal $\beta_{x}^{*}$ & $0.02 \mathrm{~m}$ \\
Vertical $\beta_{y}^{*}$ & $0.1 \mathrm{~m}$ \\
Horizontal equilibrium emittance $\varepsilon_{x}$ & $6.82 \times 10^{-8} \mathrm{~m}$ \\
Vertical emittance $\varepsilon_{y}$ & $1.36 \times 10^{-8} \mathrm{~m}$ \\
Beam size at IP $\sigma_{x}^{*}$ & $3.69 \times 10^{-5} \mathrm{~m}$ \\
Beam size at IP $\sigma_{y}^{*}$ & $3.69 \times 10^{-5} \mathrm{~m}$ \\
Momentum spread $\delta_{p}$ & $1.58 \times 10^{-3}$ \\
Momentum compaction $\alpha_{c}$ & $2.07 \times 10^{-3}$ \\
Horizontal damping time $\tau_{x}$ & $1.18 \times 10^{-3} \mathrm{sec}$ \\
Longitudinal damping time $\tau_{s}$ & $5.92 \times 10^{-4} \mathrm{sec}$ \\
\hline \hline
\end{tabular}

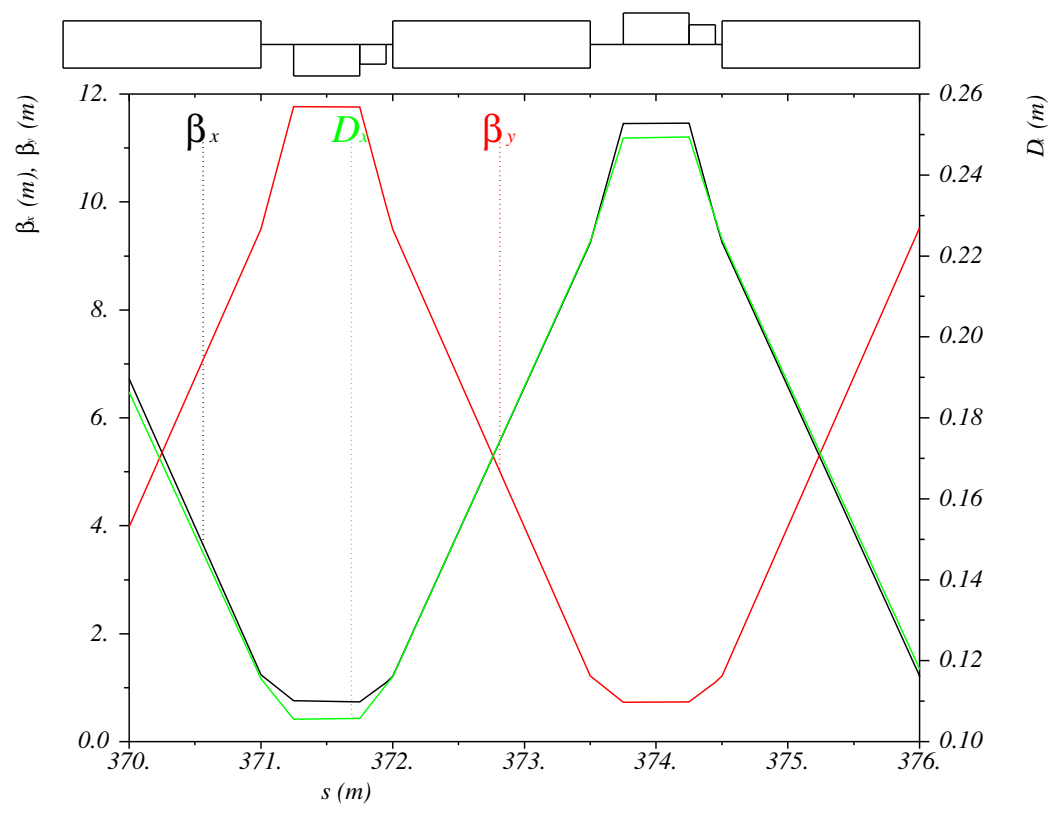

FIG. 9: Twiss function of MEIC $135^{\circ}$ arc cell 


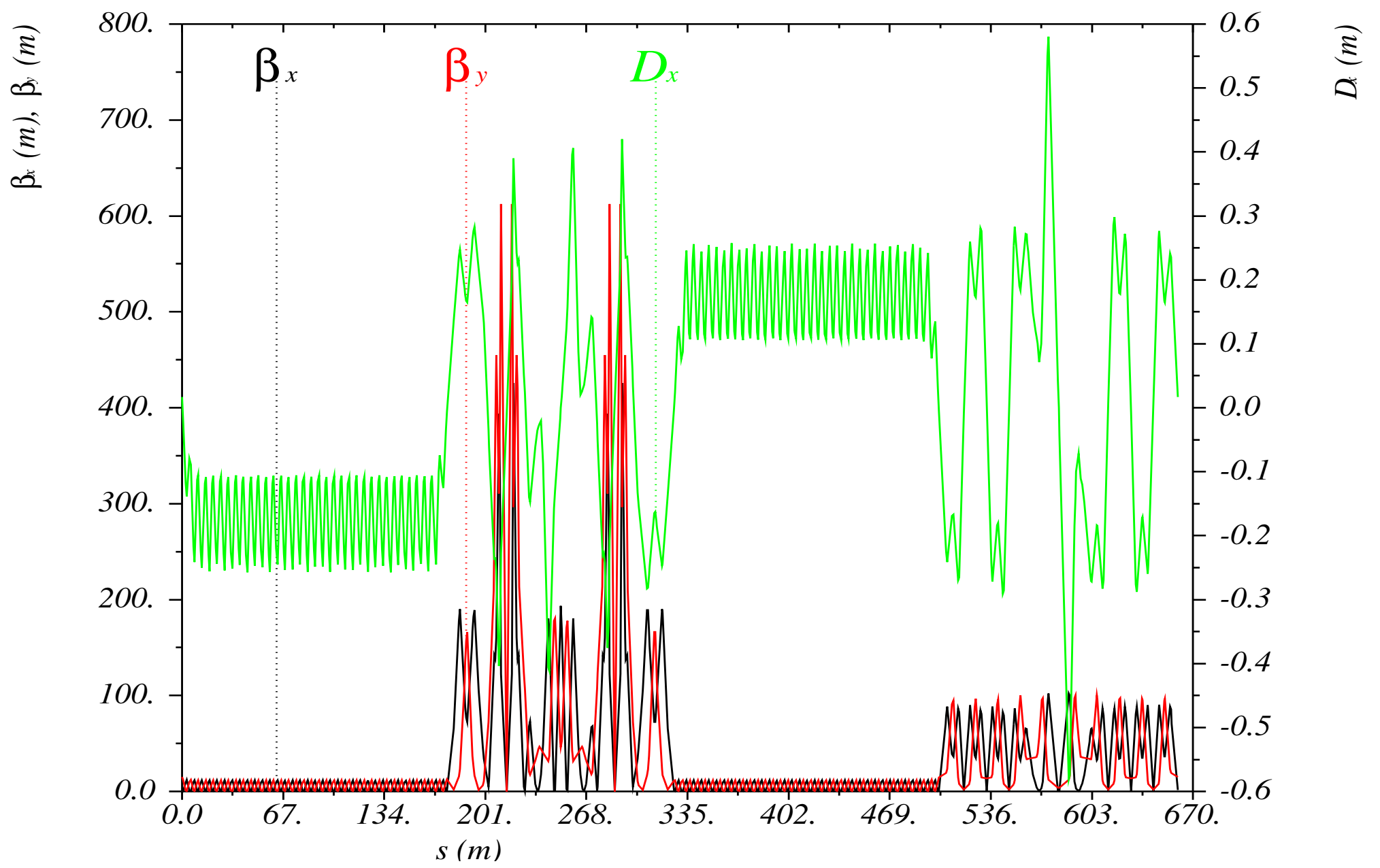

FIG. 10: Twiss functions of MEIC $135^{\circ}$ figure-8 ring. 


\section{Lattice With $90^{\circ}$ Phase Advance Cells}

In this section we explore a different arc lattice, which is inspired by the merits of SuperB design [14]. Four cells of the magnetic lattice are shown in Fig. (11) with their $\beta$-function and dispersion. Each arc cell is composed of three quadrupole magnets for alternating focusing and a horizontal bending dipole. The magnet parameters are given in Table (6). The basic structure in the two arcs is the same except that the dipoles bend beam in the reverse direction to form the figure- 8 . The two straight sections are composed of the same FODO cells, plus a matching section, and an interaction region.

TABLE 6: Magnet parameters of a MEIC $90^{\circ}$ cell.

\begin{tabular}{lccc}
\hline \hline Magnet & $L[\mathrm{~m}]$ & $\theta[\mathrm{rad}]$ & $K\left[\mathrm{~m}^{-2}\right]$ \\
\hline QUADRUPOLE & 0.560 & 0.0 & -0.138 \\
QUADRUPOLE & 0.365 & 0.0 & -0.062 \\
QUADRUPOLE & 0.365 & 0.0 & 0.260 \\
DIPOLE ARC I & 5.0996 & 0.0436 & 0 \\
DIPOLE ARC II & 5.0996 & -0.0436 & 0 \\
\hline \hline
\end{tabular}

The total path length of the figure- 8 ring is $3037.5 \mathrm{~m}$. The horizontal equilibrium emittance and other lattice parameters are given in Table (7). The choice of $90^{\circ}$ horizontal phase advance per cell was made to obtain the required low horizontal equilibrium emittance and at the same time to be able to construct a $-I$ transformation required for correcting chromatic aberrations generated by sextupole magnets. The $90^{\circ}$ lattice has an advantage in terms of chromaticity correction over the $135^{\circ}$ lattice. The former requires weaker quadrupole magnets, which in turn generates smaller natural chromaticity and ultimately it has better dynamic aperture. This last statement will be examined quantitatively in Section III.2. The dispersion function is well tailored to add chromatic correction sextupoles at places with appropriate horizontal versus vertical $\beta$ aspect ratio. Twiss functions $(\beta, \eta)$ for the figure- 8 arc are shown in Fig. (10), and the footprint is shown in Fig. (13). 
TABLE 7: Parameters of a MEIC $90^{\circ}$ lattice.

\begin{tabular}{lc}
\hline \hline Quantity & Value \\
\hline Beam energy $E$ & $9 \mathrm{GeV}$ \\
Particles/bunch $N$ & $3.1 \times 10^{10}$ \\
Bunch length $\sigma_{s}$ & $5 \times 10^{-3} \mathrm{~m}$ \\
Energy loss/turn $U_{0}$ & $8 \mathrm{MeV}$ \\
Horizontal $\beta_{x}^{*}$ & $0.02 \mathrm{~m}$ \\
Vertical $\beta_{y}^{*}$ & $0.1 \mathrm{~m}$ \\
Horizontal emittance $\varepsilon_{x}$ & $4.075 \times 10^{-8} \mathrm{~m}$ \\
Vertical emittance $\varepsilon_{y}$ & $8.15 \times 10^{-9} \mathrm{~m}$ \\
Beam size $\sigma_{x}^{*}$ & $2.85 \times 10^{-5} \mathrm{~m}$ \\
Beam size $\sigma_{y}^{*}$ & $2.85 \times 10^{-5} \mathrm{~m}$ \\
Momentum spread $\delta_{p}$ & $2.85 \times 10^{-3}$ \\
Momentum compaction $\alpha_{c}$ & $2.86 \times 10^{-3}$ \\
Horizontal damping time $\tau_{x}$ & $1.78 \times 10^{-2} \mathrm{sec}$ \\
Longitudinal damping time $\tau_{s}$ & $8.9 \times 10^{-3} \mathrm{sec}$ \\
\hline \hline
\end{tabular}

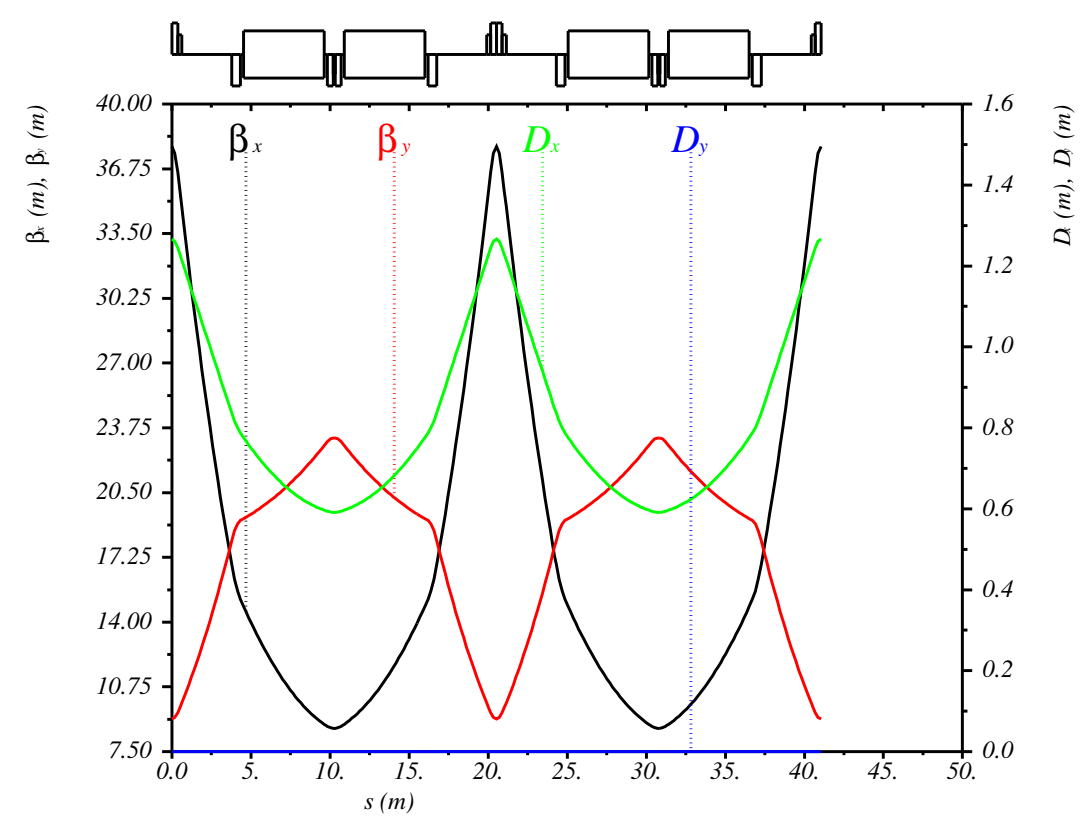

FIG. 11: Twiss functions of MEIC $90^{\circ}$ arc cell 


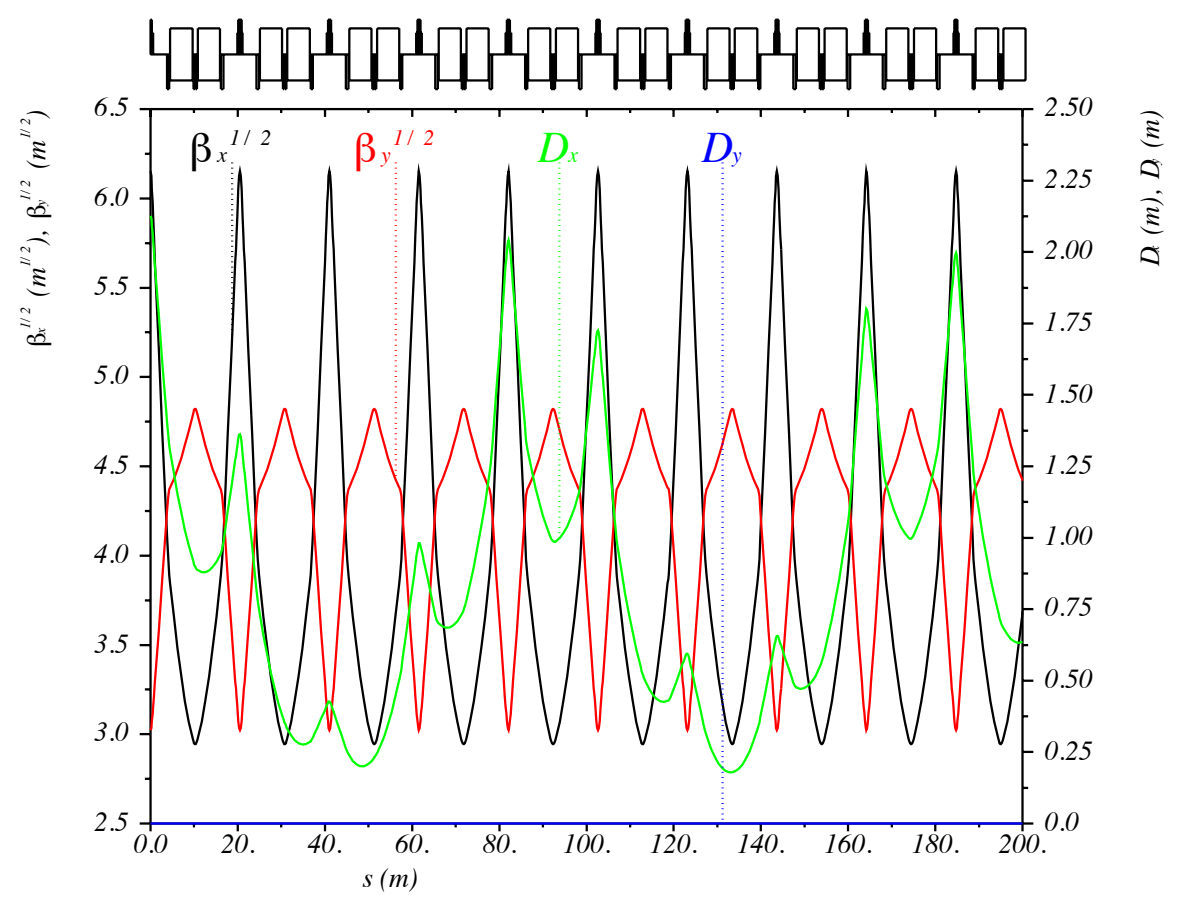

FIG. 12: MEIC $90^{\circ}$ Twiss functions

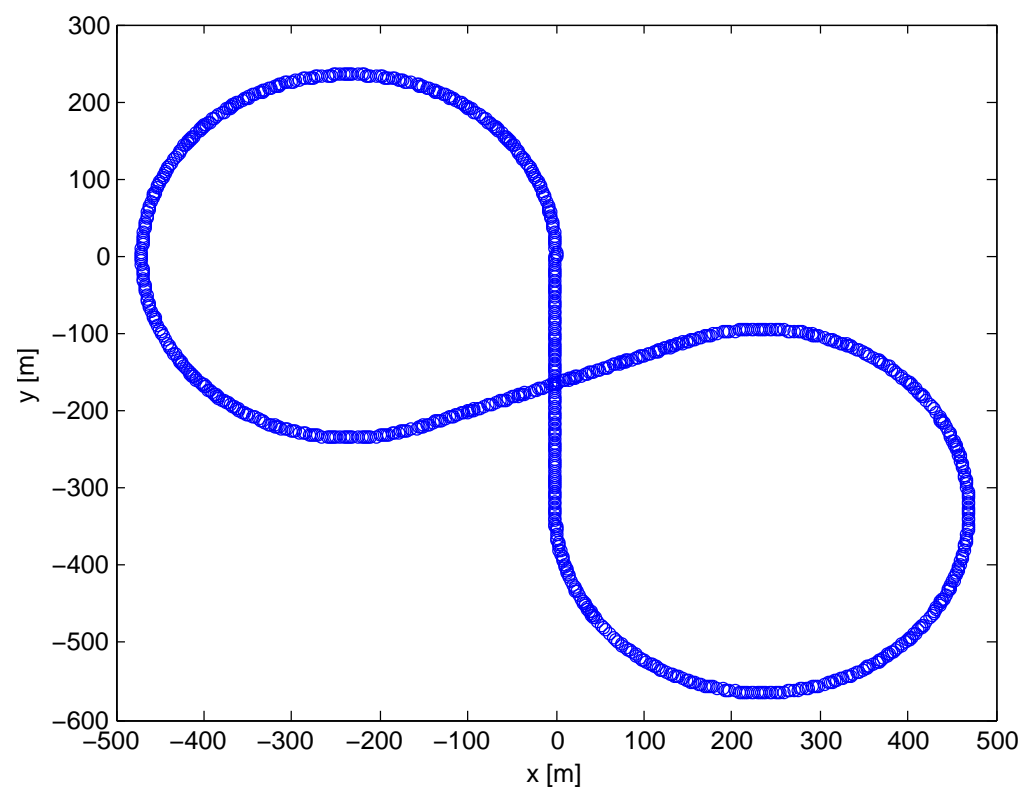

FIG. 13: Footprint of MEIC $90^{\circ}$ lattice 


\section{I.4.2 Dispersion in the straight sections}

In the two versions of the ring lattices, a dispersion free section is required to accommodate the superconducting radio frequency accelerating cavities. One of the two straight sections has been already reserved for the interaction region (Straight Section I), so the SRF cavities have to be placed along the second straight section (Straight Section II). In the following section we will discuss the treatment of dispersion in the two straight sections from the point of view of linear lattice design.

\section{Dispersion in straight section I}

The dispersion in this section is required to fulfill a number of conditions:

1 The dispersion function has to be suitable to work with local chromaticity correcting sextupoles around the IP.

2 The dispersion function has to vanish at the interaction point, but the derivative of the dispersion can be non vanishing at IP.

3 The beam size including dispersion in the beam extension section inside the final focus quadrupoles has to clear the magnet aperture limit.

These requirements are met in the linear optics design of straight section I. In this thesis two different design concepts, having different values of the of dispersion derivative at interaction point, will be evaluated and discussed in terms of chromatic correction. In the first case we use the dispersion wave generated by the arc and correct it to produce zero dispersion at interaction point with non-vanishing dispersion derivative. This design has a number of features which enable local chromaticity correction in the IR. This issue will be discussed and quantified in section III.4. In terms of linear lattice design, it is advantageous to apply this scheme in order to make the interaction region more compact. Leaving the dispersion generated by the arc section without suppressing it saves the space occupied by a dispersion suppressor. The case of non vanishing dispersion derivative at IP was introduced in a lattice with two symmetric interaction regions placed in the same straight section. The symmetric interaction regions are separated by a matching section. The matching section includes a set of symmetric dipoles which generate anti-symmetric dispersion at the two interaction regions. Fig. (14) shows dispersion through straight section I. 


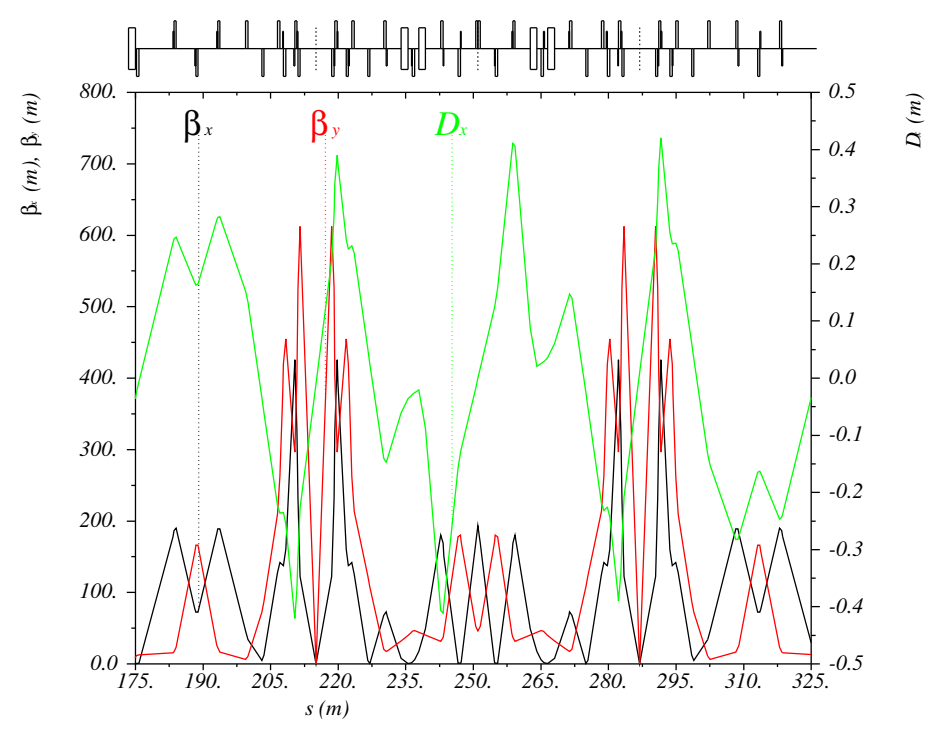

FIG. 14: Dispersion in straight section I (non - vanishing dispersion derivative at IR)

In the second case we use a matching section to connect the dispersion function along with other Twiss functions to those at the beginning of the local chromatic correction section. In this case both dispersion and its derivative are made to vanish at the interaction point. A set of dipoles is used to tailor the dispersion to be symmetric around the interaction point, while maintaining a suitably high value to work with local IR sextupoles in correcting chromaticity. Fig. (15) shows the dispersion through straight section I. This scheme features zero dispersion function inside the final focus quadrupoles; the beam size will not be a concern in the beam extension section.

\section{Dispersion in straight section II}

This straight section is reserved for SRF cavities, in addition to a single spin rotator. Dispersion has to be suppressed in straight section II. The dispersion suppressor consists of the arc's last two FODO cells following the focusing quadrupole. The bending magnet strengths are changed to suppress the dispersion, where each couple of magnets will have bending angle $\theta_{1,2}$ given by [15]

$$
\theta_{1}+\theta_{2}=\theta,
$$




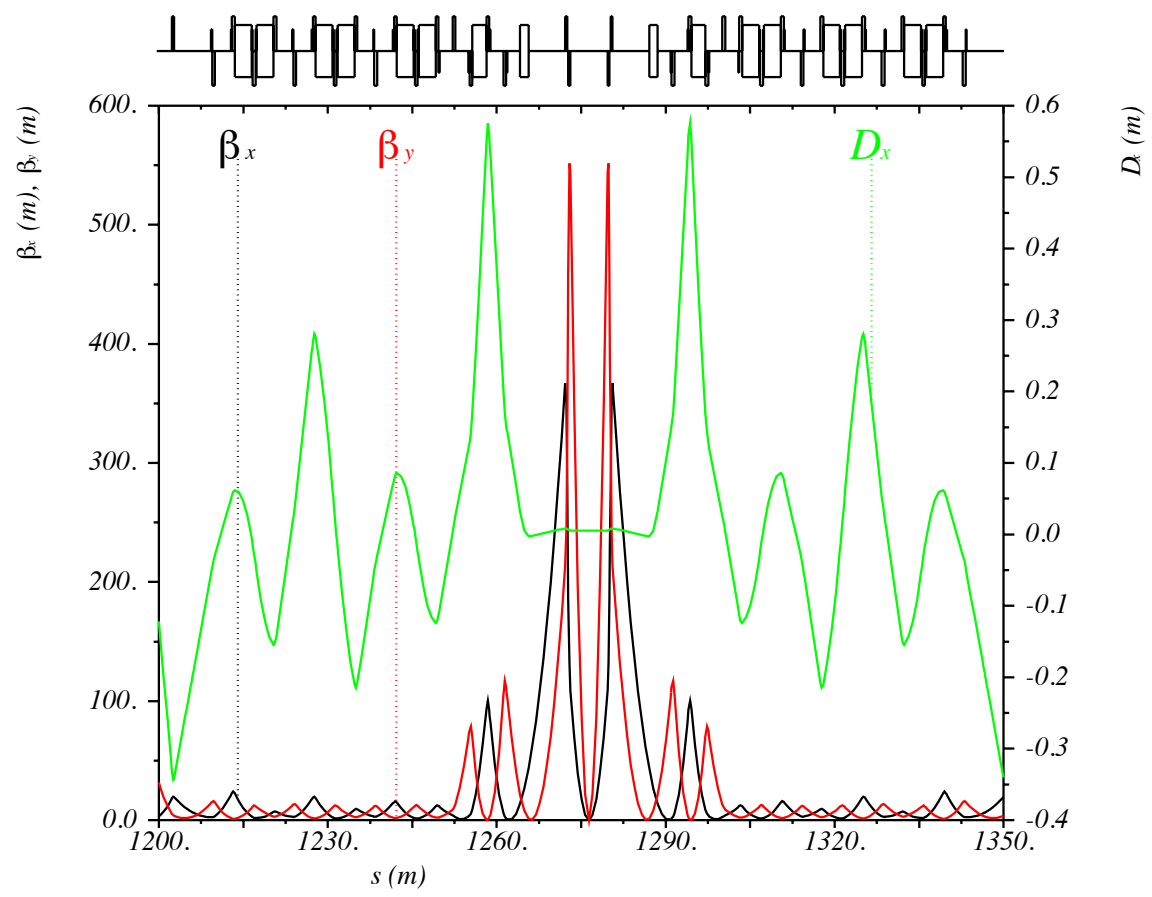

FIG. 15: Dispersion in straight section I (dispersion and its derivative vanish at IR)

where $\theta$ is the original arc dipole bending angle,

$$
\theta_{1}=\theta\left(1-1 /\left(4 \sin ^{2} \mu\right)\right)
$$

and

$$
\theta_{2}=\theta\left(1 /\left(4 \sin ^{2} \mu\right)\right)
$$

and where $\mu$ is the phase advance per regular FODO half cell. For $\mu=90$ we get $\theta_{1}=\frac{1}{2} \theta$ and $\theta_{2}=\frac{1}{2} \theta$. Fig. (17) shows suppressed dispersion in $90^{\circ}$ lattice. 


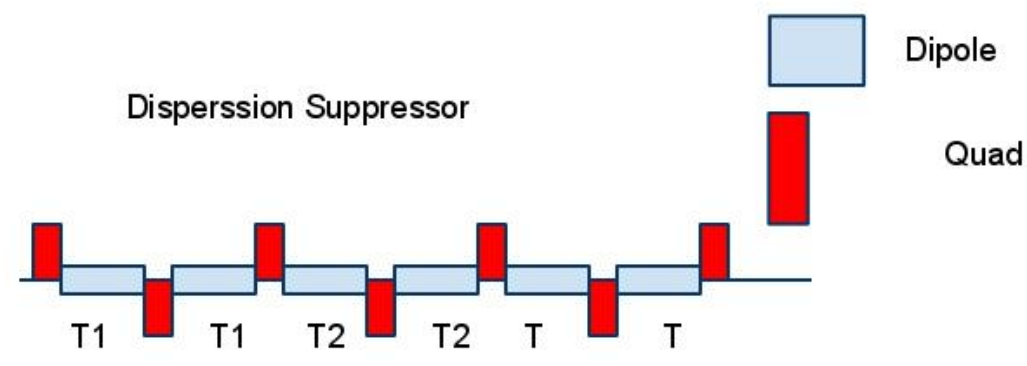

FIG. 16: Schematic of dispersion suppressor; $\left(T, T_{1}, T_{2}\right)$ are dipoles with bending angles $\left(\theta, \theta_{1}, \theta_{2}\right)$ respectively

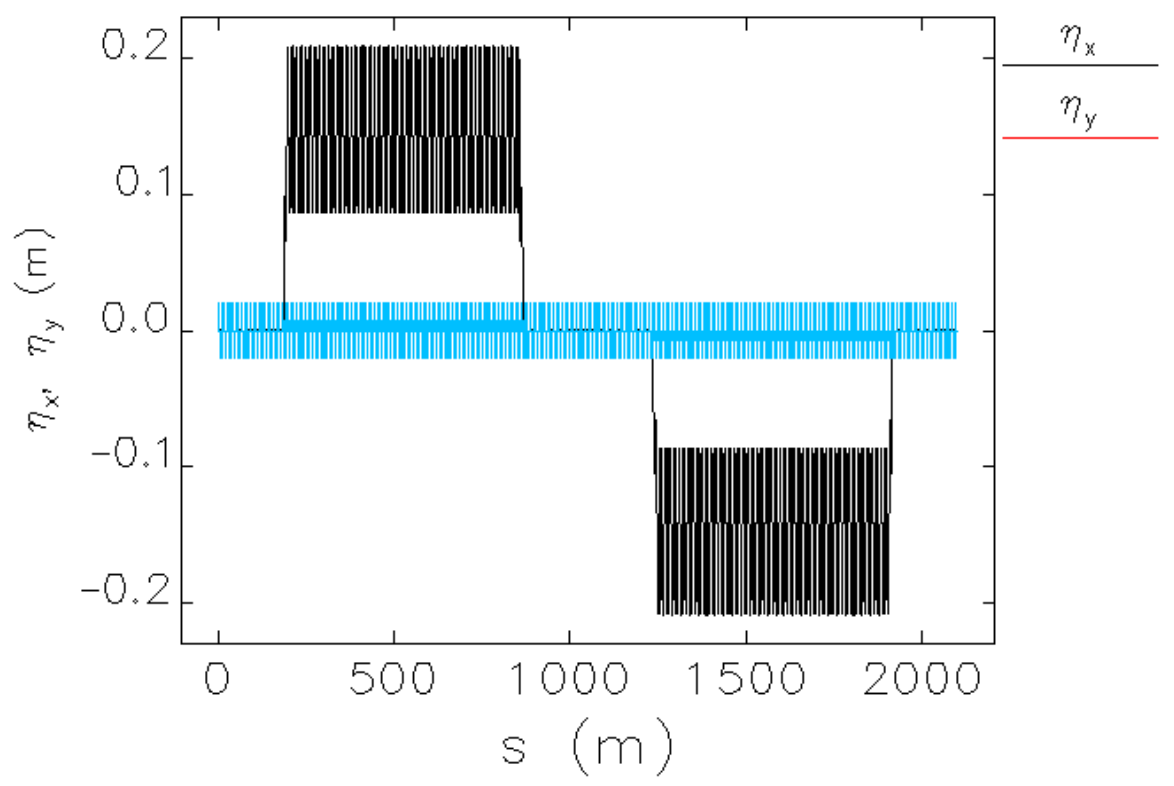

FIG. 17: Dispersion suppressed in straight section II 


\section{I.4.3 Interaction region}

The MEIC interaction region (IR) design is primarily driven by the detector needs for very demanding forward detection architecture. The corresponding detector layout is illustrated in Fig. (19).

The central detector [13] features a solenoid with endcaps on each side; it is constructed out of three main components. Two conical sections exist on each side to track particles that end up in the endcaps, a radial part includes a complete set of detectors for tracking, identification, and calorimetry, as in Fig. (18).

The IR design assumes a 7 meter distance between the interaction point (IP) and first final focus quadrupole for the ion's beamline and 3.5 meter stand off for the electron's beamline. A small crossing angle of $3^{\circ}$ requires interleaved quadrupole arrangement for both sets of final focus quadrupoles; a pair of doublets for the electrons and a triplet for the ions. Both IR designs assume $\beta_{x}^{*}$ and $\beta_{y}^{*}$ are $10 \mathrm{~cm}$ and $2 \mathrm{~cm}$ respectively.

In this section the interaction region design for two different cases will be discussed; the first case has non-vanishing dispersion derivative at IP and the second case has a vanishing dispersion derivative at the IP.

\section{Non vanishing dispersion derivative at the IP}

The electron beam interaction region is composed of a set of final focus quadrupole doublets, beam extension area, chromaticity correction block and a set of matching quadrupoles.

The distance from the IP to the final focus quadrupoles is set to $3.5 \mathrm{~m}$, which plays a role in limiting the maximum $\beta$-function values to $650 \mathrm{~m}$. This version of the lattice exhibits two mirror symmetric interaction regions placed along the same straight. Each interaction region has a mixed symmetry across the IP; final focus magnets are symmetric while the quadrupole sets at the chromaticity correction block are not symmetric, but rather exhibit symmetry with the far half of the second interaction region.

A set of two dipole magnets is placed at the end of the first IR, used to bring the dispersion wave to vanish at the two IPs, and match with the arc's periodic 


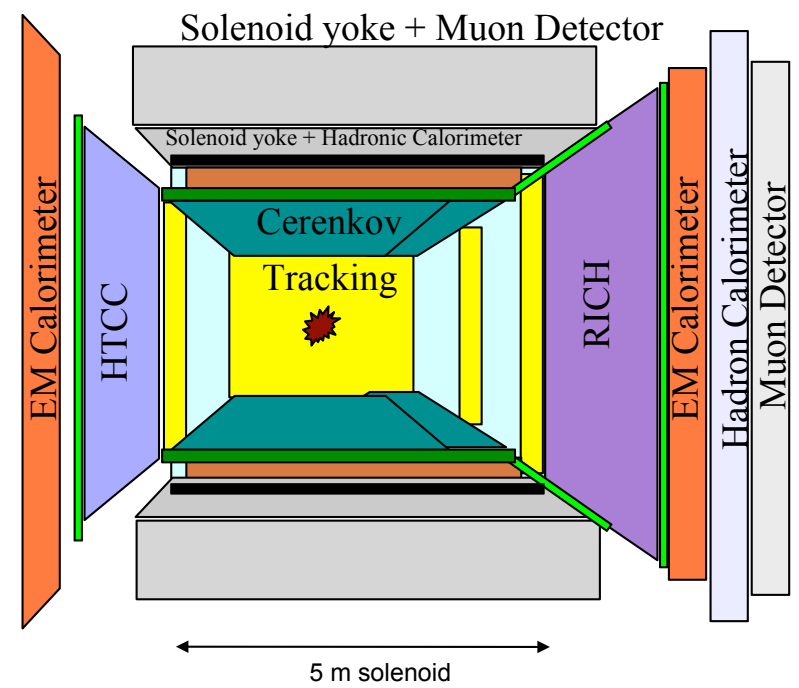

FIG. 18: Schematic of MEIC central detector showing two conical sections on each side to track particles that end up in the endcaps, a radial part includes a complete set of detectors for tracking, identification, and calorimetry.

dispersion wave. The dispersion derivative $\eta_{x}^{\prime}$ has a non vanishing value at both of the IPs leading to antisymmetric dispersion across each IP and through the final focus magnets. Fig. (20) shows the Twiss functions at the interaction region, and Table (9) lists the IR magnet lattice parameters.

Having non vanishing dispersion derivative through the IP has a number of advantages, which can be listed as follows

1. The dispersion has a suitable values close to final focus quadrupoles, which enables us to correct chromaticity much closer to its major source in the final focus quadrupoles.

2. It saves the space required for the dispersion suppressor at the end of the arc, as well as the space required for generating dispersion needed for local the chromatic correction. 


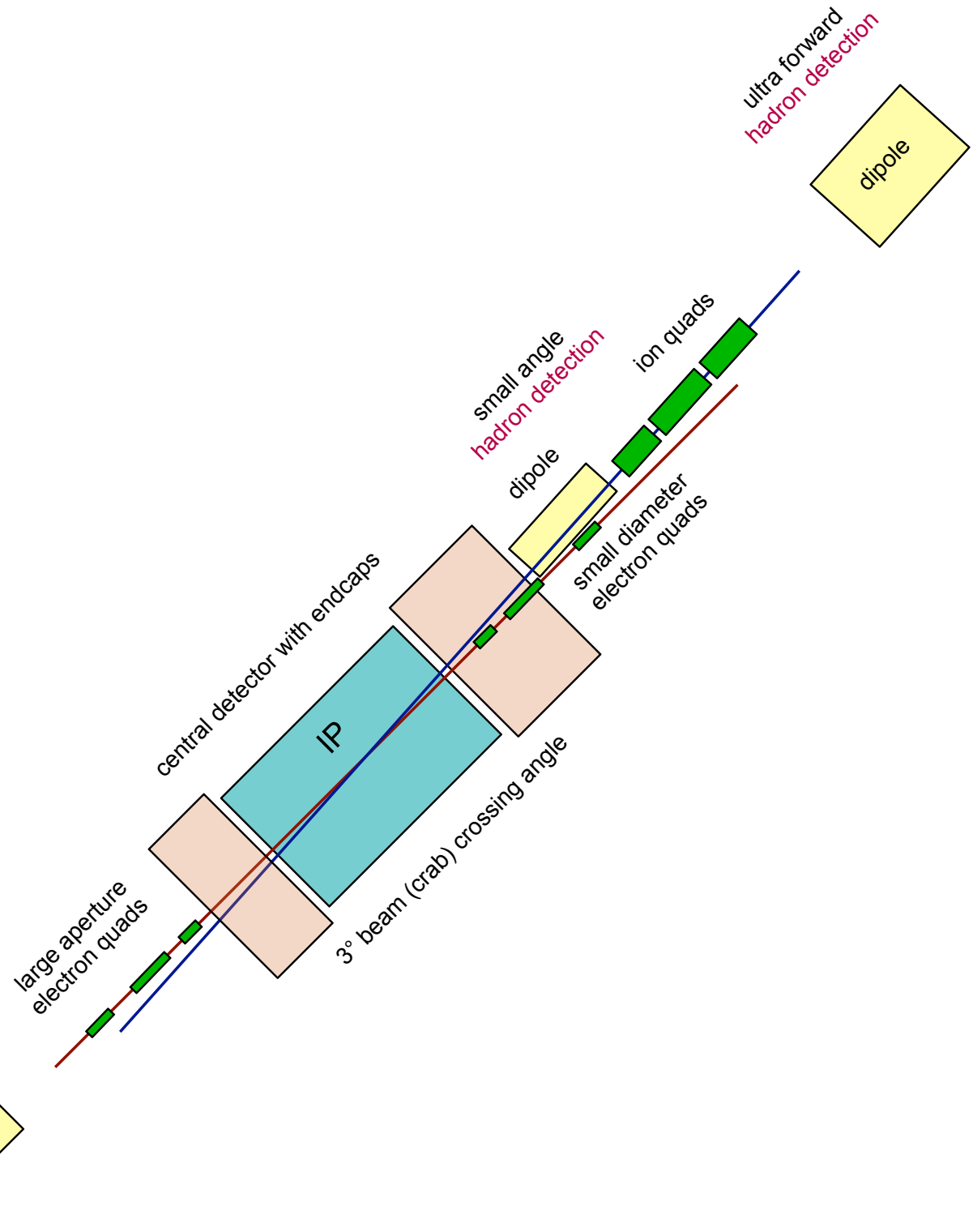

FIG. 19: Schematic of MEIC detector IR layout corresponding to $50 \mathrm{mrad}$ crossing angle 
TABLE 8: Magnet lattice parameters of a MEIC interaction region.

\begin{tabular}{lccc}
\hline \hline Lattice Element & $L[\mathrm{~m}]$ & $\theta[\mathrm{rad}]$ & $K\left[\mathrm{~m}^{-2}\right]$ \\
\hline Drift (IP to First Quad) & 3.5 & 0 & 0 \\
FF Quad I & 0.5 & 0.0 & -1.710610246 \\
FF Quad II & 0.5 & 0.0 & 1.793012185 \\
Drift (Distance between Quad I \& II) & 0.2 & 0.0 & 0.0 \\
Drift (Beam Extension) & 2.0 & 0.0 & 0.0 \\
DIPOLE I & 1.5 & -0.018 & 0 \\
DIPOLE II & 1.5 & -0.0995 & 0 \\
\hline \hline
\end{tabular}

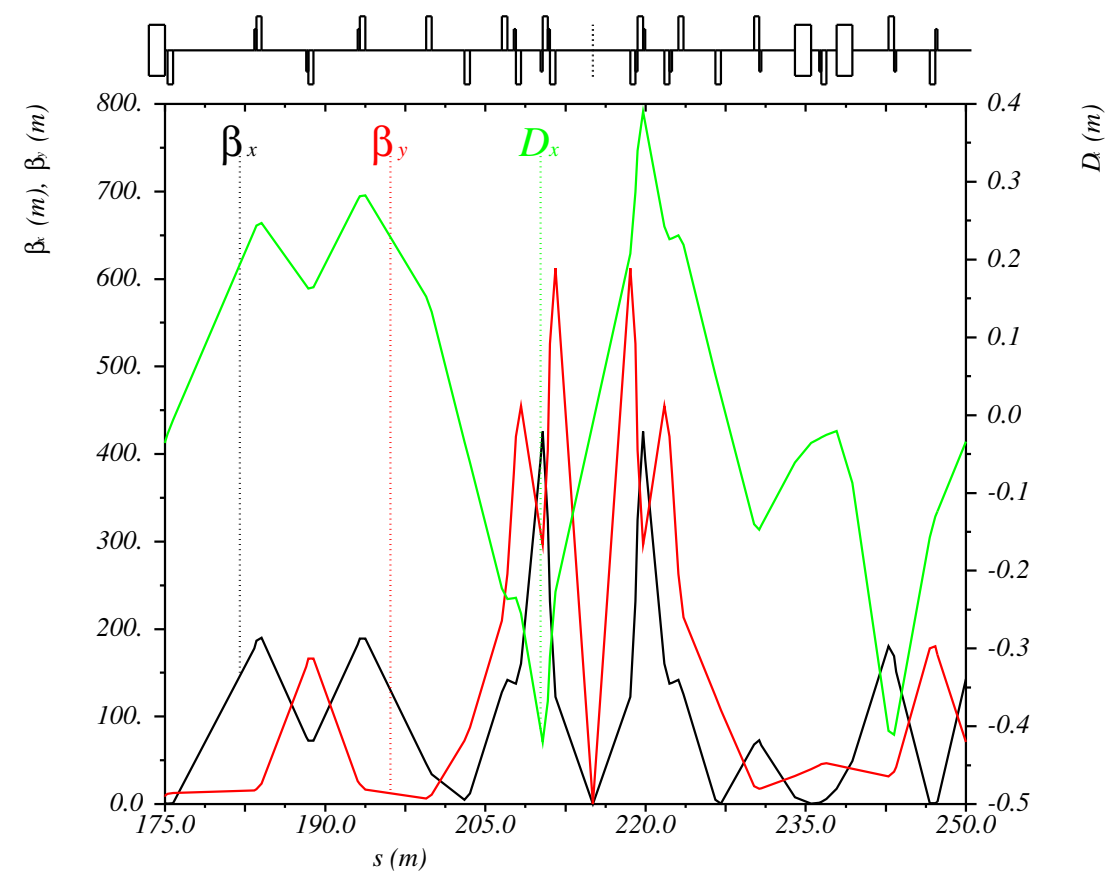

FIG. 20: Twiss parameters through the interaction region with non vanishing dispersion derivative. 


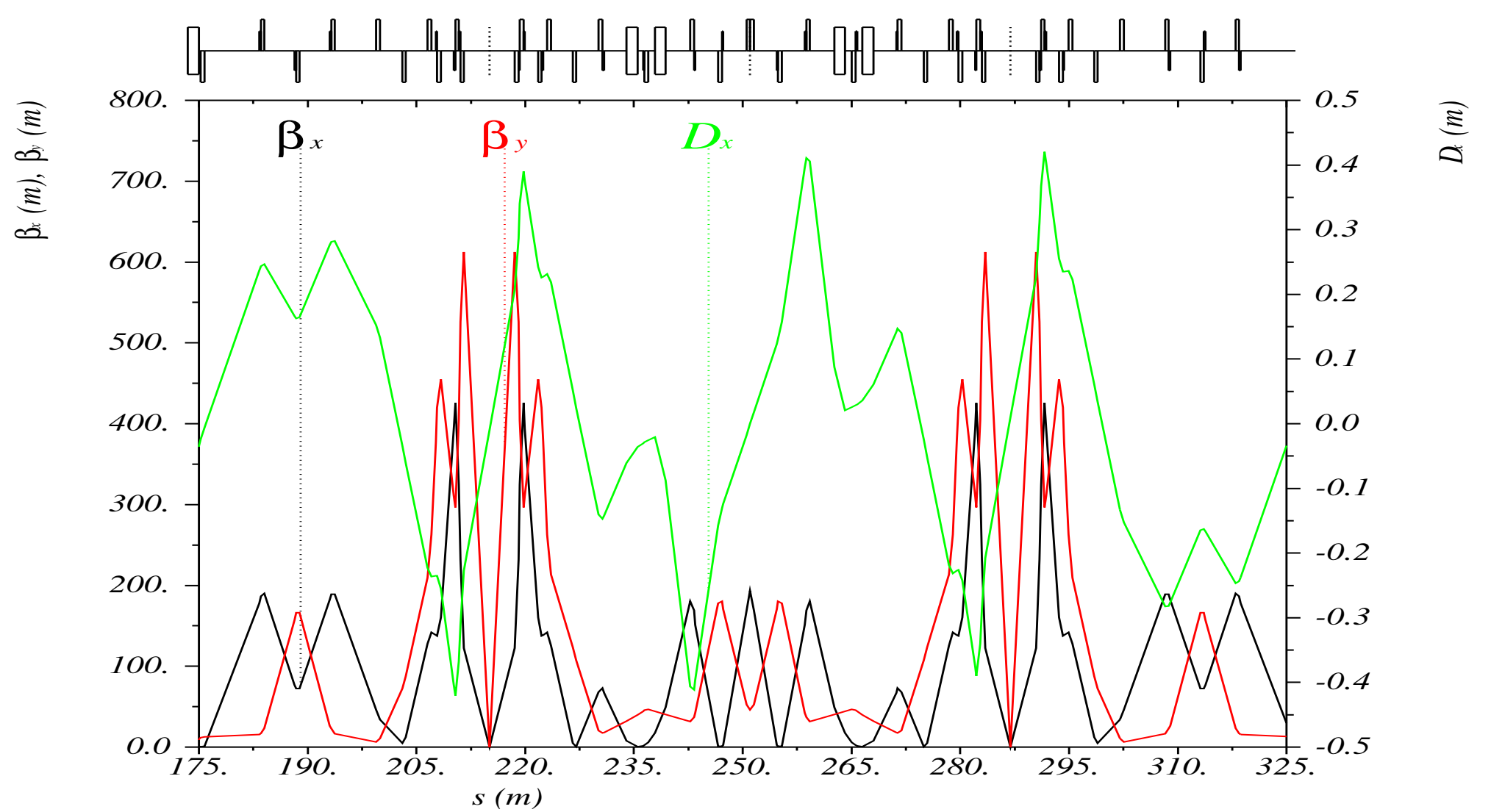

FIG. 21: Twiss parameters through two mirror symmetric IRs at the same straight with non vanishing dispersion derivative. 

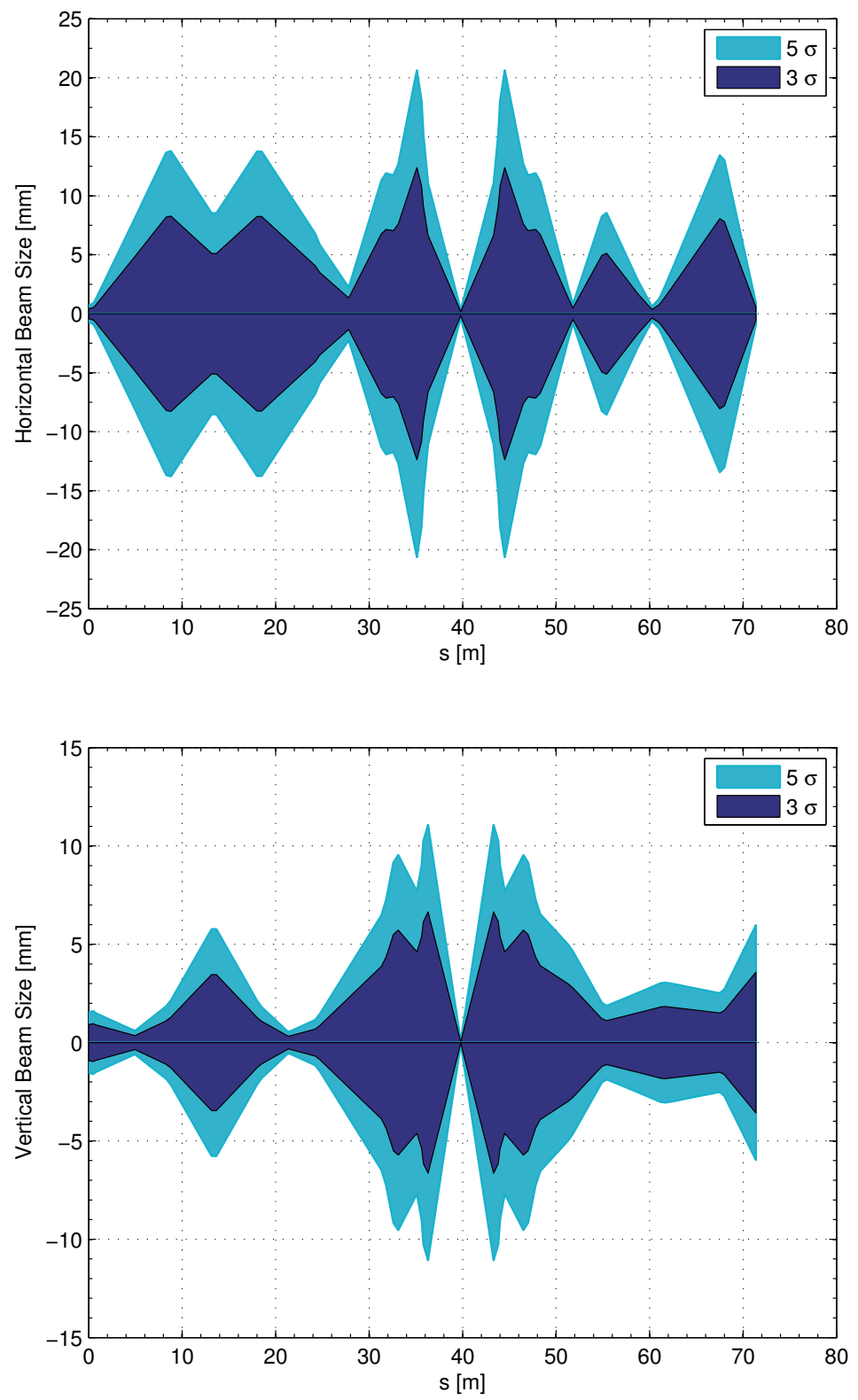

FIG. 22: Beam size at the interaction region, the horizontal beam size at IP is $8.3 \times 10^{-5} \mathrm{~m}$ and $1.7 \times 10^{-5} \mathrm{~m}$ in the vertical. The maximum amplitude is $5.38 \mathrm{~mm}$ in horizontal dimension and $2.89 \mathrm{~mm}$ in vertical dimension. 


\section{Vanishing dispersion derivative at IP}

The distance from the IP to the first final focus quadrupole is set to $3.3 \mathrm{~m}$, which plays a role in limiting the maximum $\beta$ function values to $544 \mathrm{~m}$. This version of the lattice exhibits one symmetric interaction region setting along the straight section, with a line of symmetry passing through the IP. A set of two dipole magnets placed symmetrically around the IR generates dispersion through the chromaticity compensation block and maintains vanishing dispersion and dispersion derivative at the IP. Fig. (23) shows the Twiss functions at the interaction region. Fig. (24) shows the beam size at the IR, and Table (9) lists the IR magnet lattice parameters.

TABLE 9: Magnet lattice parameters of a MEIC IR.

\begin{tabular}{lccc}
\hline \hline Lattice Element & $L[\mathrm{~m}]$ & $\theta[\mathrm{rad}]$ & $K\left[\mathrm{~m}^{-2}\right]$ \\
\hline Drift (IP to First Quad) & 3.3 & 0 & 0 \\
FF Quad I & 0.5 & 0.0 & -3.024116403 \\
FF Quad II & 0.5 & 0.0 & 2.701291167 \\
Drift (Distance between Quad I \& II) & 2.0 & 0.0 & 0.0 \\
Drift (Beam Extension) & 6.5 & 0.0 & 0.0 \\
DIPOLE I & 1.5 & 0.05 & 0 \\
\hline \hline
\end{tabular}

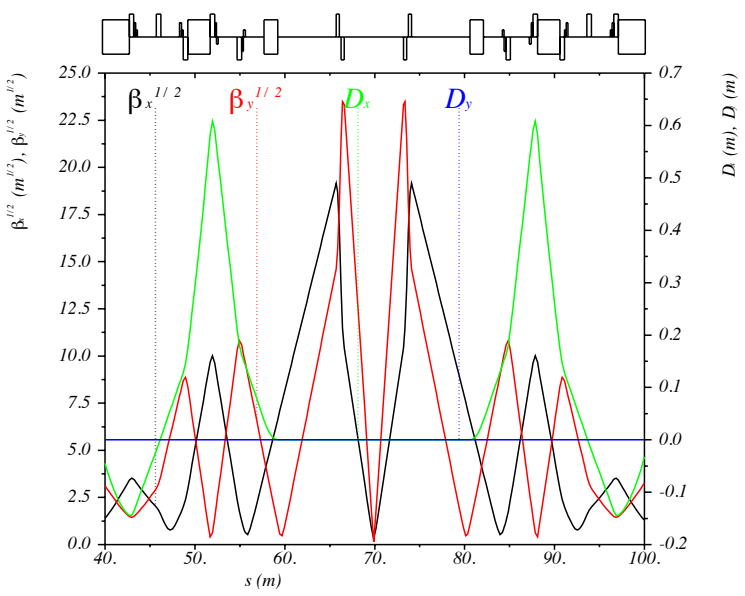

FIG. 23: Twiss parameters through interaction region with vanishing dispersion derivative at the interaction point. 

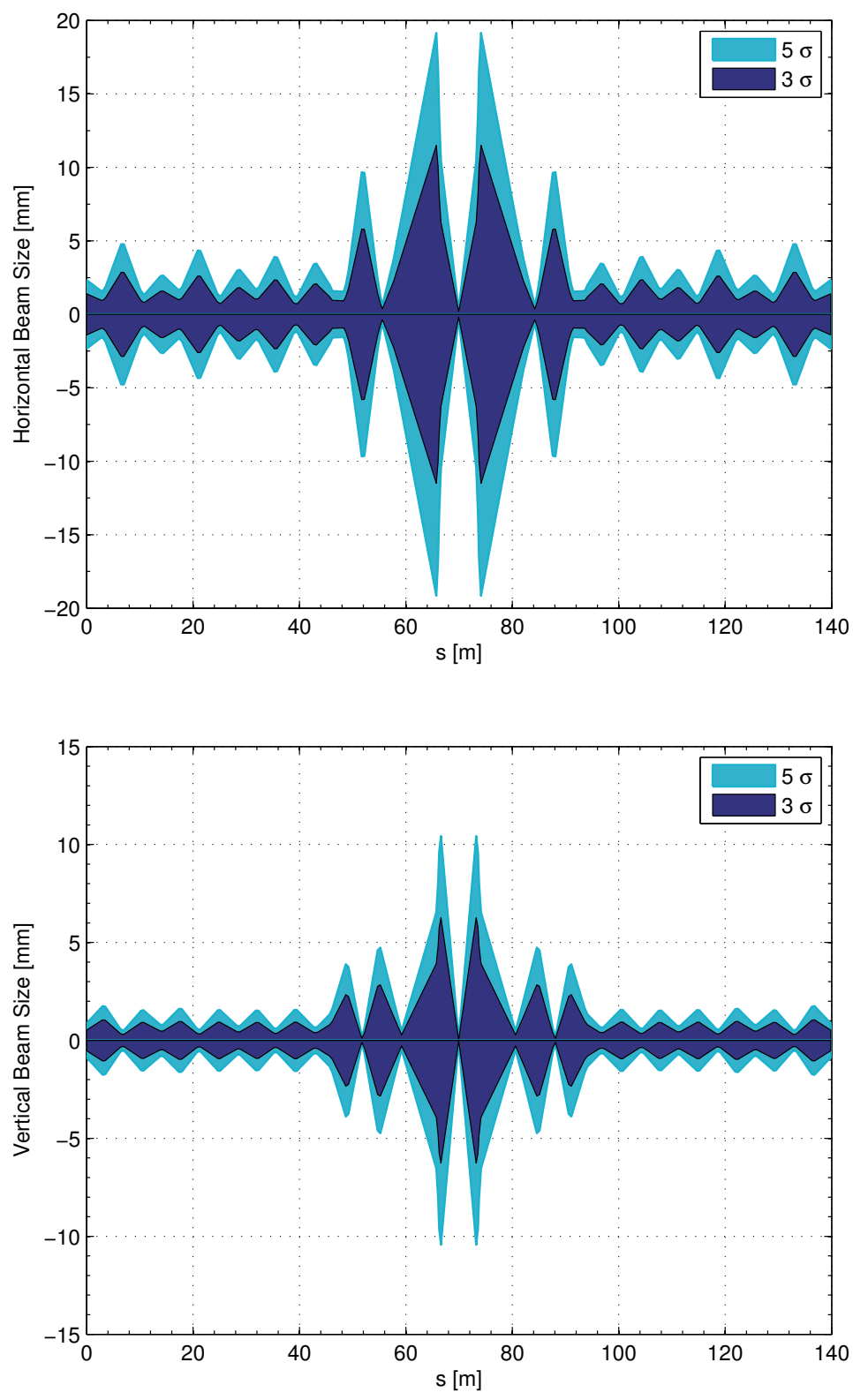

FIG. 24: Beam size at the interaction region, the horizontal beam size at interaction point is $2.8 \times 10^{-5} \mathrm{~m}$ and $1.26 \times 10^{-5} \mathrm{~m}$ in the vertical. The maximum amplitude is $3.8 \mathrm{~mm}$ in horizontal dimension and $2.08 \mathrm{~mm}$ in vertical dimension. 


\section{I.4.4 Spin rotators}

A set of spin rotators is needed to meet MEIC requirements of preserving the polarization and producing longitudinal polarization vector at the interaction point (IP). The rotators have been designed conceptually and involve solenoid magnets to perform the spin rotation. In this thesis, a novel design of a decoupling compensator is presented which balances out the effects of the solenoids in the spin rotators. A discussion with more details about MEIC polarization and spin rotator design will be given in Chapter IV.

\section{I.5 SUMMARY}

In this chapter an introduction to MEIC and its requirements were presented. The MEIC design parameters were shown in Table (3). A set of lattice design goals was introduced here, as well as some linear electron ring designs which satisfy the requirements. Further details regarding compensation for chromaticity, polarization, and coupling will be given in the next chapters.

A brief basic summary of the HERA electron-proton collider design was presented. The HERA electron ring lattice was constructed out of $60^{\circ}$ phase advance cells. The HERA rings were $6.4 \mathrm{~km}$ long, such a large ring made it possible to produce the required small equilibrium emittance. In the MEIC/ELIC design the ring size is limited to $0.6-3 \mathrm{~km}$, which mandates the use of larger phase advance per cell in order to produce the required small equilibrium emittance. The chromaticity generated from HERA's arc quadrupoles and final focus quadrupoles are much smaller than the chromaticity expected from MEIC, due to the stronger arc and final focus quadrupoles used in MEIC's design. In the HERA lepton beams were polarized by making use of Sokolov-Ternov effect to raise the polarization of the injected unpolarized lepton beams up to $30-45 \%$. In contrast at MEIC, highly polarized electron beam is produced at CEBAF and injected into MEIC with up to $80 \%$ polarization. 


\section{CHAPTER II}

\section{CHROMATICITY AND CHROMATICITY CORRECTION}

\section{II.1 INTRODUCTION}

A collection of particles within a circulating accelerator bunch has a distribution of energies above and below the design nominal energy $E_{o}$. In this thesis the case of circular accelerators with separated function lattices is considered. In this case the magnitude of the momentum deviation is defined by the equilibrium energy spread which depends only on the particle energy, bending radius, and the ring optics. Such energy deviations lead to focusing errors in the quadrupole magnets which cause particles with different energies to be focused at different focal points and potentially causing a smearing effect at the focused beam spot. The smearing effect at the IP is of great importance for collider accelerators such as MEIC and it can be one of the major reduction factors of the luminosity of the collider. The quadrupole focusing errors in the lattice will introduce deviations from the nominal machine tunes. These deviations are functions of the relative momentum deviation.

In the following two sections the effects of momentum deviation on the beam spot size at the interaction point of the collider will be discussed. The effect on the tune of circular accelerators will also be discussed in more detail.

\section{II.2 HILL'S EQUATIONS}

To introduce the chromaticity generated by a quadrupole magnet due to energy deviations in the passing beam, we will start with the Hamiltonian which describes motion of a charged particle in an electromagnetic field, and then we will drive the equation of motion in quadrupole magnet. Once the equations of motion are known we will be able to calculate small perturbations due to the energy deviations.

By using the pathlength as an independent variable in preference to time, the Hamiltonian for a charged particle in an external electromagnetic field is given by [16] 


$$
\begin{aligned}
& H\left(x, p_{x}, y, p_{y},-p_{t}, c t ; s\right)=-[1+K(s) x] \\
& \quad \times\left[\frac{q}{p_{o}} A_{s}(s)+\sqrt{\left.1-\left[p_{x}-\frac{q}{p_{o}} A_{x}(s)\right]^{2}-\left[p_{y}-\frac{q}{p_{o}} A_{y}(s)\right]^{2}-\frac{2 p_{t}}{\beta}+p_{t}^{2}\right]},\right.
\end{aligned}
$$

where the momenta $p_{x, y}$ are normalized by the nominal momentum $p_{0}, s$ is the pathlength, $\beta$ is the relativistic factor, and $p_{t}$ and $K$ are defined by

$$
p_{t}=-\frac{\delta E}{p_{o} c}
$$

and

$$
K(s)=\frac{1}{\rho(s)},
$$

where $\rho(s)$ is the horizontal radius of curvature.

For a particle with a given momentum deviation, $\delta \equiv\left(p-p_{0}\right) / p_{0}$, the canonical transformation generated by

$$
\begin{gathered}
F_{2}=\frac{c t}{\beta}\left[1-\sqrt{1+\beta^{2}\left(2 \delta+\delta^{2}\right)}\right], \\
H_{2}=H_{1}+\frac{\partial F_{2}}{\partial s}, \\
-c T=\frac{\partial F_{2}}{\partial \delta}=-\frac{\beta(1+\delta) c t}{\sqrt{1+\beta^{2}\left(2 \delta+\delta^{2}\right)}}, \\
p_{t}=\frac{\partial F_{2}}{\partial(c t)}=\frac{1}{\beta}\left[1-\sqrt{1+\beta^{2}\left(2 \delta+\delta^{2}\right)}\right]
\end{gathered}
$$

where

$$
t=T \frac{\sqrt{1+\beta^{2}\left(2 \delta+\delta^{2}\right)}}{\beta(1+\delta)},
$$

and

$$
1-\frac{2}{\beta} p_{t}+p_{t}^{2}=(1+\delta)^{2}
$$

simplifies the equations of motion. The new Hamiltonian takes the following form:

$$
\begin{gathered}
H\left(x, p_{x}, y, p_{y}, \delta, c t ; s\right)=-[1+K(s) x] \times\left[\frac{q}{p_{o}} A_{s}(s)+\right. \\
\left.\sqrt{(1+\delta)^{2}-\left[p_{x}-\frac{q}{p_{o}} A_{x}(s)\right]^{2}-\left[p_{y}-\frac{q}{p_{o}} A_{y}(s)\right]^{2}}\right]
\end{gathered}
$$


For times that are short compared to the synchrotron frequency the adiabatic approximation, where the momentum deviation can be viewed as a slowly varying parameter, may be applied. Using the multipole expansion of the vector potential in the source-free region with piece-wise constant fields, one expands the Hamiltonian to third order in the phase space coordinates [17].

$$
H\left(x, p_{x}, y, p_{y} ; s\right)=-[1+K(s) x]\left[1+\delta-\frac{p_{x}^{2}+p_{y}^{2}}{2(1+\delta)}+\frac{q}{p_{o}} A_{s}(s)\right]+O(4) .
$$

The multipole expansion of the vector potential $\left(A_{x}, A_{y}, A_{s}\right)$ in a source-free region is used in Eq. (36) to yield [18]:

$$
\begin{aligned}
& A_{x}(s)=0, \\
& A_{y}(s)=0, \\
& \frac{q}{p_{o}} A_{s}(s)=-\operatorname{Re}\left[\sum_{n=1}^{\infty} \frac{1}{n}\left[b_{n}(s)+i a_{n}(s)\right](x+i y)^{n}\right],
\end{aligned}
$$

where $b_{n}(s)\left(a_{n}(s)\right)$ are the normal (skew) multipole coefficients. The $n$-index represents the $2 n$ pole (e.g. $b_{3}$ is the normal sextupole coefficient with a dimension of $(\text { length })^{-2}$ ).

The magnetic field can be calculated by

$$
\begin{aligned}
B_{x}(s) & =\frac{1}{1+K(s) x} \frac{\partial A_{y}}{\partial s}-\frac{\partial A_{s}}{\partial y}, \\
B_{y}(s) & =\frac{K(s)}{1+K(s) x} A_{s}-\frac{\partial A_{s}}{\partial x}-\frac{1}{1+K(s) x} \frac{\partial A_{x}}{\partial s}, \\
B_{s}(s) & =\frac{\partial A_{x}}{\partial y}-\frac{\partial A_{y}}{\partial x} .
\end{aligned}
$$

When $K(s)=0$, the corresponding magnetic fields are given by

$$
B_{y}(s)+i B_{x}(s)=-\frac{p_{o}}{q} \sum_{n=1}^{\infty}\left[b_{n}(s)+i a_{n}(s)\right](x+i y)^{n-1} .
$$

Assuming a piece-wise constant field magnetic lattice consisting of dipoles with cylindrical geometry and quadrupoles and sextupoles with Cartesian geometry, and considering the case where the local radius of curvature is small, the Hamiltonian takes the following form [17]:

$$
\begin{array}{r}
H\left(x, p_{x}, y, p_{y} ; s\right)=\frac{p_{x}^{2}+p_{y}^{2}}{2(1+\delta)}-b_{1}(s) x \delta+\frac{b_{1}^{2}(s)}{2} x^{2}+ \\
\frac{b_{2}(s)}{2}\left(x^{2}-y^{2}\right)+\frac{b_{3}(s)}{3}\left(x^{3}-3 x y^{2}\right)+O(4)
\end{array}
$$


Finally, the equations of motion derived from Hamilton's equations are:

$$
\begin{aligned}
x^{\prime} & =\frac{\partial H}{\partial p_{x}}=\frac{p_{x}}{1+\delta}+O(3) \\
p_{x}^{\prime} & =-\frac{\partial H}{\partial x}=b_{1}(s) \delta-\left(b_{1}^{2}(s)+b_{2}(s)\right) x-b_{3}(s)\left(x^{2}-y^{2}\right)+O(3), \\
y^{\prime} & =\frac{\partial H}{\partial p_{y}}=\frac{p_{y}}{1+\delta} \\
p_{y}^{\prime} & =-\frac{\partial H}{\partial y}=b_{2}(s) y+2 b_{3}(s) x y+O(3)
\end{aligned}
$$

Combining Hamilton's equations of motion into two second order ordinary differential equations yields Hill's equations.

$$
\begin{aligned}
& x^{\prime \prime}+\frac{b_{2}(s)+b_{1}^{2}(s)}{1+\delta} x=b_{1}(s) \delta-\frac{b_{3}(s)}{1+\delta}\left(x^{2}-y^{2}\right)+O(3), \\
& y^{\prime \prime}-\frac{b_{2}(s)}{1+\delta} y=\frac{2 b_{3}(s)}{1+\delta} x y+O(3),
\end{aligned}
$$

where $b_{1}=1 / \rho . b_{2}$ and $b_{3}$ are the quadrupole and sextupole normal fields respectively.

\section{II.3 TUNE CHROMATICITY IN COLLIDER RINGS}

As seen in Section I.1.2, the betatron tune of a circular accelerator is defined as the number of betatron oscillations the particles accumulate as they travel once around the ring, and is given by the following formula

$$
\xi=\frac{1}{4 \pi} \int_{s_{0}}^{s_{0}+L} \frac{1}{\beta} d s,
$$

where $\beta$ is the betatron Twiss function. The average value of the betatron function around the ring can be approximatley given by

$$
\bar{\beta}=\frac{R}{\xi},
$$

where $R$ is the radius of the ring $R=L /(2 \pi)$

A deviation in a particle energy from the design energy leads to a change in the tune of the particle. This dependence is known as the chromaticity. In the following section, we will derive the first order chromaticity and later the second order expansion with regard to energy deviation $\delta$. 


\section{II.3.1 First order chromaticity}

From Eq. (42) considering the case of only quadrupole and sextupole magnets and setting $b_{1}(s)=0$, and $1 /(1+\delta) \approx(1-\delta+\ldots)$, Hill's equations will take the following form:

$$
\begin{aligned}
& x^{\prime \prime}+b_{2}(s) x(1-\delta)=-b_{3}(1-\delta)\left(x^{2}-y^{2}\right), \\
& y^{\prime \prime}-b_{2}(s) y(1-\delta)=2 b_{3}(1-\delta) x y
\end{aligned}
$$

We need to retain only the betatron oscillation terms involving $x_{\beta}$ or $y_{\beta}$ to derive chromatic tune shifts. Setting $x=x_{\beta}+\eta_{x} \delta$, and $y=y_{\beta}$, where $\eta_{y}=0$, to account for dispersion,

$$
\begin{aligned}
& x^{\prime \prime}+b_{2}(s) x(1-\delta)=-\frac{b_{3}(s)}{2}(1-\delta)\left(x^{2}-y^{2}\right), \\
& y^{\prime \prime}-b_{2}(s) y(1-\delta)=b_{3}(s)(1-\delta) x y,
\end{aligned}
$$

and therefore

$$
\begin{aligned}
& x_{\beta}^{\prime \prime}+b_{2}(s) x_{\beta}=b_{2}(s) x_{\beta} \delta-b_{3}(s) \eta_{x} x_{\beta} \delta-\frac{b_{3}(s)}{2}\left(x_{\beta}^{2}-y_{\beta}^{2}\right)+O(3), \\
& y_{\beta}^{\prime \prime}-b_{2}(s) y_{\beta}=-b_{2}(s) y_{\beta} \delta+b_{3}(s) \eta_{y} y_{\beta} \delta+b_{3}(s) x_{\beta} y_{\beta}+O(3) .
\end{aligned}
$$

Ignoring the nonchromatic terms of the second order (geometric aberrations),

$$
\begin{aligned}
& x_{\beta}^{\prime \prime}+b_{2}(s) x_{\beta}=\left(b_{2}(s)-b_{3}(s) \eta_{x}\right) x_{\beta} \delta \\
& y_{\beta}^{\prime \prime}-b_{2}(s) y_{\beta}=-\left(b_{2}(s)-b_{3}(s) \eta_{y}\right) y_{\beta} \delta
\end{aligned}
$$

we observe that the perturbation terms are linear in the betatron amplitude

$$
\begin{aligned}
\xi_{x} & =-\frac{1}{4 \pi} \oint \beta_{x}\left(b_{2}(s)-b_{3}(s) \eta_{x}\right) d s, \\
\xi_{y} & =\frac{1}{4 \pi} \oint \beta_{y}\left(b_{2}(s)-b_{3}(s) \eta_{x}\right) d s .
\end{aligned}
$$

A more accurate derivation of the first and higher order chromaticity based on a more exact formula for the nonlinear dispersion function was introduced in [19]. The expansion of the tune, $\xi$, with respect to the momentum deviation, $\delta$, is given by,

$$
\xi=\sum_{n=0} \delta^{n} \xi_{n}
$$

where

$$
\xi_{x 1}=\frac{1}{4 \pi} \int_{s_{0}}^{s_{0}+L}\left[-\beta_{x}\left(K_{x}^{2}+b_{2}-b_{3} \eta_{0}\right)-2 \alpha_{x} K_{x} \eta_{0}^{\prime}+\gamma_{x} K_{x} \eta_{0}\right] d s
$$




$$
\xi_{y 1}=\frac{1}{4 \pi} \int_{0}^{s} d s\left(\beta_{y}\left(b_{2}-b_{3} \eta_{0}\right)+\gamma_{y} K_{x} \eta_{0}\right),
$$

and $\gamma_{x}$ is defined as

$$
\gamma_{x}=\left(K_{x}^{2}+b_{2}\right) \beta_{x}+\frac{1}{2} \beta_{x}^{\prime \prime}
$$

\section{II.3.2 Second order tune chromaticity}

A perturbative formula for the nonlinear dispersion function of a ring, which gives explicit expressions for higher-order terms, was derived in [20]. The derivation starts from the Hamiltonian without linearizing the kinematic term and then gives recursion expressions for higher order terms up to the fourth order. The nonlinear dispersion was used to derive the higher order expressions for the nonlinear chromaticity.

The second order variation of the tune, $\xi_{2}$, with respect to the momentum deviations, $\delta$, is given by [19]

$$
\xi_{x 2}=\frac{1}{4 \pi} \int_{s_{0}}^{s_{0}+L}\left[G_{x, 2}(s)-\frac{1}{16} \mu_{0} a_{1}^{2}(0)-\sum_{n=1}^{\infty} \frac{\mu_{0}^{3}}{8\left(\mu_{0}^{2}-\pi^{2} n^{2}\right)}\left[c_{1}^{2}(n)+d_{1}^{2}(n)\right]\right] d s
$$

where

$$
\begin{array}{r}
G_{x, 2}=\beta_{x}\left[K_{x}^{2}\left(1-\frac{1}{4}\left(\eta_{0}^{\prime}\right)^{2}\right)-K_{x}^{3} \eta_{0}+\frac{1}{2} K_{x}^{4} \eta_{0}^{2}+b_{2}-b_{3}\left(\eta_{0}-\eta_{1}\right)+\frac{1}{2} b_{4} \eta_{0}^{2}\right] \\
-2 \alpha_{x}\left(K_{x} \eta_{1}^{\prime}-K_{x}^{2} \eta_{0} \eta_{0}^{\prime}\right)+\gamma_{x}\left(K_{x} \eta_{1}-\frac{1}{2} K_{x}^{2} \eta_{0}^{2}+\frac{3}{2} \eta_{0}^{2}\right) .
\end{array}
$$

The integral form of the Fourier components $c_{1}(n)$ and $d_{1}(n)$ are given by:

$$
\begin{array}{r}
c_{1}(n)=\frac{2}{\mu_{0}} \int_{s_{0}}^{s_{0}+L}\left[\left(G_{x, 1}-\frac{1}{2 \beta_{x}}\left(\frac{2 \pi n}{\mu_{x}}\right)^{2} K_{x} \eta_{0}\right) \cos \left[\frac{2 \pi n}{\mu_{x}} \phi_{x}\right]+\right. \\
\left.\frac{2 \pi n}{\mu_{x}}\left(\frac{\alpha_{x}}{\beta_{x}} K_{x} \eta_{0}-K_{x} \eta_{0}^{\prime}\right) \sin \left[\frac{2 \pi n}{\mu_{x}} \phi_{x}\right]\right] d s \\
d_{1}(n)=\frac{2}{\mu_{0}} \int_{s_{0}}^{s_{0}+L}[ \\
\quad\left(G_{x, 1}-\frac{1}{2 \beta_{x}}\left(\frac{2 \pi n}{\mu_{x}}\right)^{2} K_{x} \eta_{0}\right) \sin \left[\frac{2 \pi n}{\mu_{x}} \phi_{x}\right]- \\
\left.\frac{2 \pi n}{\mu_{x}}\left(\frac{\alpha_{x}}{\beta_{x}} K_{x} \eta_{0}-K_{x} \eta_{0}^{\prime}\right) \cos \left[\frac{2 \pi n}{\mu_{x}} \phi_{x}\right]\right] d s .
\end{array}
$$

$G_{x, 1}$ is given by

$$
G_{x, 1}=-\beta_{x}\left(K_{X}^{2}+b_{2}-b_{3} \eta_{0}\right)-2 \alpha_{x} K_{x} \eta_{0}^{\prime}+\gamma_{x} K_{x} \eta_{0}
$$




\section{II.4 CHROMATICITY IN CASE OF A STRAIGHT BEAMLINE}

Next, the first and second order chromaticity for a straight section will be discussed. This special case is interesting for the MEIC, where the interaction region is the major contributor to chromaticity and it is located in a straight section. Chromaticity for different configurations will be presented in the following order: drift space, pure quadrupole field, pure sextupole field, and finally pure octupole field.

Let us consider the first case of a drift with no magnetic fields by setting $b_{2}=$ $b_{3}=b_{4}=0$ in Eqs. (51) and (54). We have:

$$
\xi_{1}=0
$$

and

$$
\xi_{2}=\frac{1}{4 \pi} \int_{0}^{l}\left(\frac{3}{4} \eta_{0}^{2} \beta^{\prime \prime}\right) d s .
$$

In case of a pure quadrupole field by setting $b_{3}=b_{4}=0$ in Eqs. (51) and (54), we have:

$$
\xi_{1}=\frac{1}{4} \int_{0}^{l}\left[\beta_{x} \eta_{0} b_{3}\right] d s
$$

where the second order chromaticity will take the following form:

$$
\begin{array}{r}
\xi_{2}=\frac{1}{4 \pi} \int_{0}^{l}\left[\beta_{x} b_{2}+\frac{3}{2} \eta_{0}^{2}\left(b_{2} \beta_{x}+\frac{\beta_{x}^{\prime \prime}}{2}\right)\right] d s-\frac{1}{8}\left(\int_{0}^{l}\left(\beta_{x} b_{2}\right) d s\right)^{2}+ \\
\sum_{n=1}^{\infty} \frac{\mu_{x}}{2\left(\mu_{x}^{2}-\pi^{2} n^{2}\right)}\left[\left(\int_{0}^{l} \beta_{x} b_{2} \cos \left(\frac{2 \pi n}{\mu_{x}} \phi_{x}\right) d s\right)^{2}+\left(\int_{0}^{l} \beta_{x} b_{2} \sin \left(\frac{2 \pi n}{\mu_{x}} \phi_{x}\right) d s\right)^{2}\right] .
\end{array}
$$

For a pure sextupole field, by setting $b_{2}=b_{4}=0$ in Eqs. (51) and (54), we have

$$
\xi_{1}=\frac{1}{4 \pi} \int_{0}^{l} \beta_{x} \eta_{0} b_{3} d s,
$$

where the second order chromaticity will take the following form:

$$
\begin{array}{r}
\xi_{2}=\frac{1}{4 \pi} \int_{0}^{l}\left[-\beta_{x} b_{3}\left(\eta_{0}-\eta_{1}\right)+\frac{3}{4} \eta_{0}^{2} \beta_{x}^{\prime \prime}\right] d s-\frac{1}{8}\left(\int_{0}^{l}\left(\beta_{x} \eta_{0} b_{3}\right) d s\right)^{2}+ \\
\sum_{n=1}^{\infty} \frac{\mu_{x}}{2\left(\mu_{x}^{2}-\pi^{2} n^{2}\right)}\left[\left(\int_{0}^{l} \beta_{x} \eta_{0} b_{3} \cos \left(\frac{2 \pi n}{\mu_{x}} \phi_{x}\right) d s\right)^{2}+\left(\int_{0}^{l} \beta_{x} \eta_{0} b_{3} \sin \left(\frac{2 \pi n}{\mu_{x}} \phi_{x}\right) d s\right)^{2}\right] .
\end{array}
$$

The last case considered here is for a pure octupole field. Here the first order vanishes and the second order will take the following form:

$$
\xi_{2}=\frac{1}{4 \pi} \int_{0}^{l} \frac{\eta_{0}^{2}}{2}\left(\beta_{x} b_{4}+\frac{3}{2} \beta_{x}^{\prime \prime}\right) d s .
$$




\section{II.4.1 Compensating chromaticity in a straight interaction region}

In this section we will introduce the conditions for canceling the first and second order chromaticity, using a combined function magnet with quadrupole, sextupole, and octupole components. This conceptual case will help in understanding the general idea of compensating chromaticity in collider rings. The equations indicate that the first order chromaticity is excited by a combined function magnet having three multipole components (quadrupole, sextupole, and octupole):

$$
\xi_{x 1}=\frac{1}{4 \pi} \int_{0}^{l} \beta_{x}\left(-b_{2}+\eta_{0} b_{3}\right) d s .
$$

We can see that the choice of sextupole field, $b_{3}=b_{2} / \eta_{0}$ will compensate for the first order chromaticity.

Now considering the second order chromaticity in our conceptual case, the second order chromaticity will be reduced to the following form:

$$
\begin{array}{r}
\xi_{2}=\frac{1}{4 \pi} \int_{0}^{l}\left[\beta_{x}\left(b_{2}-b_{3}\left(\eta_{0}-\eta_{1}\right)\right)+\frac{1}{2} \beta_{x} b_{4} \eta_{0}^{2}+\frac{3}{2} \eta_{0}^{2}\left(b_{2} \beta_{x}+\frac{\beta_{x}^{2}}{2}\right)\right] d s \\
-\frac{1}{8}\left(\int_{0}^{l} \beta_{x}\left(b_{2}-b_{3} \eta_{0}\right) d s\right)^{2}+\sum_{n=1}^{\infty} \frac{\mu_{x}}{2\left(\mu_{x}^{2}-\pi^{2} n^{2}\right)} \\
{\left[\left(\int_{0}^{l} \beta_{x}\left(b_{2}-b_{3} \eta_{0}\right) \cos \left(\frac{2 \pi n}{\mu_{x}} \phi_{x}\right) d s\right)^{2}+\left(\int_{0}^{l} \beta_{x}\left(b_{2}-b_{3} \eta_{0}\right) \sin \left(\frac{2 \pi n}{\mu_{x}} \phi_{x}\right) d s\right)^{2}\right]}
\end{array}
$$

by setting $b_{3}=b_{2} / \eta_{0}$, we see cancelations in the oscillatory terms and $\xi_{2}$ will take the much simpler form:

$$
\xi_{2}=\frac{1}{4 \pi} \int_{0}^{l}\left[\beta_{x} b_{2} \frac{\eta_{1}}{\eta_{0}}+\frac{3}{2} \beta_{x} b_{2} \eta_{0}^{2}+\frac{3}{4} \eta_{0}^{2} \beta_{x}^{\prime \prime}+\frac{1}{2} \beta_{x} b_{4} \eta_{0}^{2}\right] d s,
$$

where the integrand reduces to zero when setting the octupole field to the value given by

$$
b_{4}=-b_{2}\left(3+2 \frac{\eta_{1}}{\eta_{0}^{3}}\right)-\frac{3}{2} \frac{\beta_{x}^{\prime \prime}}{\beta_{x}} .
$$

From the above we can conclude that in order to compensate the first order chromaticity that originates from quadrupoles, one would need to use sextupoles at locations with non vanishing dispersion; larger dispersion would allow us to use weaker sextupoles. From Eq. (69), we see one could use octupole magnets to compensate for second order chromaticity generated by correcting sextupoles. 


\section{Amplitude dependent aberrations}

A scheme to compensate the amplitude dependent aberrations introduced by a chromaticity correcting sextupole has been proposed in [21]. The scheme involves using sextupoles in pairs, where every two sextupoles in a pair are separated by a negative identity transformation $(-I)$ in both planes;

$$
-I=\left(\begin{array}{cccc}
-1 & 0 & 0 & 0 \\
0 & -1 & 0 & 0 \\
0 & 0 & -1 & 0 \\
0 & 0 & 0 & -1
\end{array}\right)
$$

The sextupoles can be represented as thin magnets introducing kicks in the particle trajectories given by

$$
\begin{aligned}
\Delta x^{\prime} & =-\frac{1}{2} m_{0} l_{s}\left(x^{2}-y^{2}\right), \\
\Delta y^{\prime} & =-m_{0} l_{s} x y
\end{aligned}
$$

where $m$, and $l_{s}$ are the sextupole strength and length. A thin sextupole transformation matrix $M_{s}$, is given by:

$$
M_{s}\left(x_{0}, y_{0}\right)=\left(\begin{array}{cccc}
1 & 0 & 0 & 0 \\
-\frac{1}{2} m_{0} l_{s} x_{0} & 1 & \frac{1}{2} m_{0} l_{s} x_{0} & 0 \\
0 & 0 & 1 & 0 \\
0 & 0 & m_{0} l_{s} x_{0} & 1
\end{array}\right)
$$

Evaluating the complete transformation through a sextupole pair separated by $-I$ transformation yields

$$
M_{t}=M_{s}(-I) M_{s}=-I
$$

showing a complete cancellation of geometric aberrations in both horizontal and vertical planes. This correction scheme has been applied successfully to the final focus system of the Stanford Linear Collider [22]. Compensation of geometric aberrations is not perfect due to the fact that, in reality, sextupoles have finite length. One could represent finite length sextupole as a series of thin sextupole slices, where interleaved slices between every pair of slices will spoil the perfect cancelation. 


\section{II.5 BETA CHROMATICITY IN THE FINAL FOCUS}

In this section the Montague chromatic functions are introduced, which represent a useful figure of merit to quantify chromaticity in the vicinity of the interaction region (IR). It also works as a useful tool in optimizing the $\beta$-chromaticity at the final focus.

First; we start with notation. Following [23], one defines the Twiss parameters $\beta, \alpha$, and the phase advance $\phi$ for particles with momentum deviation $\delta$, and for particles with zero momentum deviation as

$$
\begin{aligned}
& \beta_{1}=\beta(0), \beta_{2}=\beta(\delta), \\
& \alpha_{1}=\alpha(0), \alpha_{2}=\alpha(\delta), \\
& \phi_{1}=\phi(0), \phi_{2}=\phi(\delta) .
\end{aligned}
$$

One defines $\Delta \beta, \Delta \alpha$, and $\Delta k$ as follows:

$$
\begin{aligned}
& \Delta \beta=\beta_{2}-\beta_{1}, \beta=\sqrt{\beta_{1} \beta_{2}}, \\
& \Delta \phi=\phi_{2}-\phi_{1}, \phi=\frac{1}{2}\left(\phi_{1}+\phi_{2}\right), \\
& \Delta k=k_{2}(\delta)-k_{1}(0),
\end{aligned}
$$

where $k$ is the focusing gradient error.

Let us define the chromatic variables as:

$$
\begin{aligned}
& A=\frac{\alpha_{2} \beta_{1}-\alpha_{1} \beta_{2}}{\sqrt{\beta_{1} \beta_{2}}}, \\
& B=\frac{\beta_{2}-\beta_{1}}{\sqrt{\beta_{2} \beta_{1}}}=\frac{\Delta \beta}{\beta} .
\end{aligned}
$$

Introduce the derivatives:

$$
\begin{aligned}
& \frac{d A}{d s}=\beta \Delta k+2 B \frac{d \phi}{d s}, \\
& \frac{d B}{d s}=2 A \frac{d \phi}{d s} .
\end{aligned}
$$

In a region with $\Delta k=0$ from Eq. (77) we find that

$$
\frac{d}{d s}\left(A^{2}+B^{2}\right)=0
$$

Therefore $\left(A^{2}+B^{2}\right)$ is invariant. The Montague chromatic function is defined as:

$$
W=\frac{1}{2}\left(A^{2}+B^{2}\right)^{\frac{1}{2}}
$$


The Montague chromatic function is a dimensionless quantity that can be used as an absolute measure of the linear chromatic perturbation in a transport line. The factor of $1 / 2$ represents the fact that the chromatic function tends to oscillate at twice the betatron frequency. It is worth mentioning that this function proves useful in optimizing required sextupole strengths for chromatic corrections, it has been implemented in the optics design codes MAD-8 and MAD-X. It is especially useful in studying local chromatic correction of low- $\beta$ insertions in the final focus of colliders.

Consider the thin lens approximation in the low- $\beta$ insertion case [23]. The chromatic function, $A$, in a single thin focusing lens at distance $s$ from the interaction point (IP) is given by:

$$
A=-\frac{2 s}{\beta^{*}}
$$

where $\beta^{*}$ is the Twiss function at the interaction point. The contribution of the final focus quadruple to Montague chromatic function, $W$, takes the form [23],

$$
W=\frac{s}{\beta^{*}} \text {. }
$$

One could calculate a rough estimate of the contribution of final focus quadrupoles to $W$ functions in both transverse planes, given that the initial $\mathrm{W}$ function is zero. In case of the MEIC, the distance between the interaction point and the first final focus quadrupole is $L=3.3 \mathrm{~m}$. In the horizontal plane, $\beta_{x}^{*}=0.10 \mathrm{~m}$ and the Montague function $W_{x}=33$, while in vertical plane $W_{y}=165$.

The Montague function represents a measure of the blur of the focused particles at the interaction point ( $\beta$-chromaticity). In the following work the $W$ functions were used as an optimization parameter to reduce $\beta$-chromaticity at the IP using local sextupoles placed close to IPs in places with a non vanishing dispersion.

\section{Chromatic increase in spot size at final focus}

Uncorrected chromaticity leads to an energy dependent increase in spot size as given by [22]. See Fig. (25),

$$
\begin{aligned}
& \frac{\Delta \sigma_{x, y}}{\sigma_{x, y}^{*}} \approx \frac{F}{\beta_{x, y}^{*}} \sigma_{E}, \\
& \frac{\Delta \sigma_{x, y}}{\sigma_{x, y}^{*}} \approx \xi_{x, y}^{*} \sigma_{E} .
\end{aligned}
$$


This increase is to be added in quadrature to the unperturbed spot size. Here $F$ is the focal length, $\sigma_{E}$ is the rms energy spread in the beam, and $\sigma_{x, y}(s)$ is the $r m s$ beam size at location $s$.

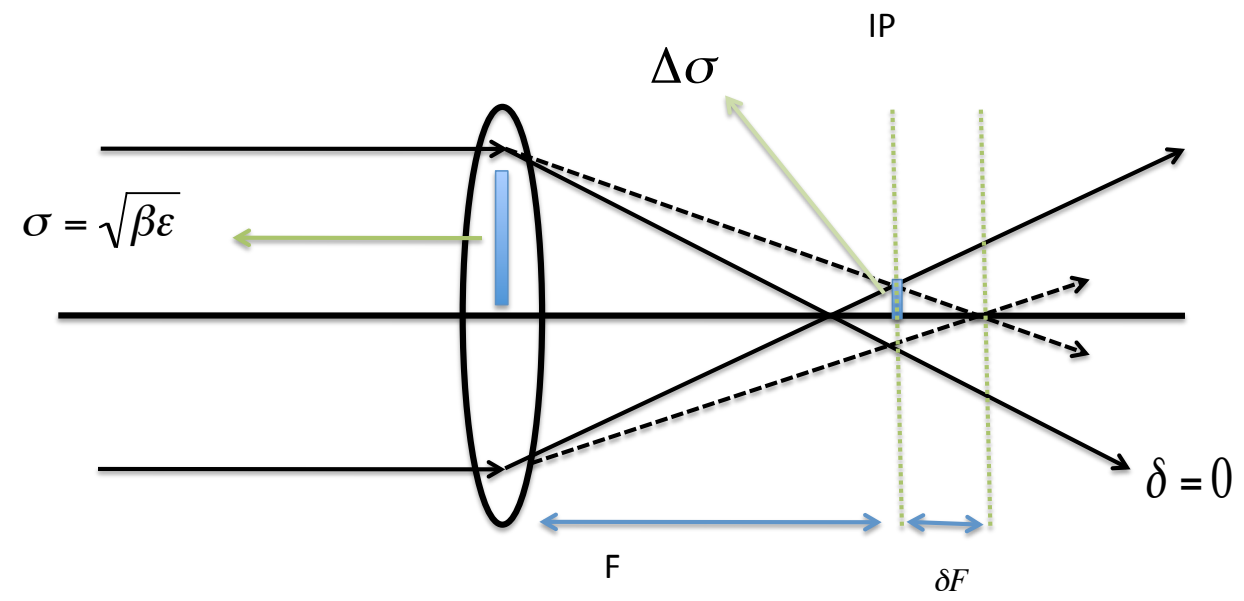

FIG. 25: Energy-dependent increase in spot size

\section{II.6 CHROMATICITY CORRECTION SCHEMES}

In this section, a summary of common chromaticity correction schemes used in linear and circular particle colliders will be presented. We will start with the global correction, which refers to correcting chromaticity via sextupole families distributed through the arcs. Then the local correction will be discussed. The local correction is carried out by a single or multiple families of sextupoles located in the vicinity of the interaction region.

\section{II.6.1 Global chromatic correction}

Most of the current storage and collider rings use a global distributed scheme in correcting chromaticity. In this scheme, a single or multiple families of sextupoles are placed within arc cells in positions with dispersion and a good aspect ratio between both transverse $\beta$-functions. As stated before, in order to compensate for geometric aberrations the phase advance between sextupoles in each family has to be set to $180^{\circ}$ which gives the required $-I$ transformation. 
The simplest scheme for correcting chromaticity in the arcs is to place a sextupole next to each quadrupole. All sextupoles close to focusing quadrupoles compose a family, SF, and those next to defocusing quadrupoles compose another family, SD. Since $\beta_{x}$ will be large for a SF family and $\beta_{y}$ will be large for a SD family, this will reduce the required sextupole strength for correcting chromaticity. Those sextupole families are sufficient to make the arc achromatic. It is worth mentioning that the closer the sextupoles are to quadrupoles, the better the chromaticity compensation. This is due to the fact that nearer to the quadrupoles the difference between horizontal and vertical betatron amplitudes increases, which helps in decoupling them when compensating tune spread. In the case of a collider ring, the major sources of chromaticity are the final focus quadrupoles. In some designs the final focus section is placed in a dispersion free region, making the sextupoles in the arcs the only resource to correct chromaticity.

\section{II.6.2 Local chromatic correction}

Modern designs for low $\beta$-insertions for particle colliders require a very small $\beta$ function to achieve high luminosity. In these cases, as the final focusing quadrupoles are the major source of chromaticity, it is more advantageous to compensate for chromaticity locally around the interaction region so that the chromatic waves do not to propagate to the arcs.

To be able to compensate chromaticity, we first need to create dispersion along the beamline close to the interaction region, keeping in mind that beam size has to be very small at the interaction point and the dispersion has to vanish there. The first derivative of the dispersion can be non-vanishing at the interaction point. A local correction scheme has been proposed for linear colliders [24, 25, 22]; later it was adopted by new circular collider designs, like the Super B-factory [14].

Local chromaticity correction has several advantages over global correction, and can be summarized in the following points:

- The local correction helps in maintaining a very small beam size at the IP, $\beta$ chromaticity has to be corrected at the IP.

- The local correction helps in maintaining a good dynamic aperture, since the $\beta$ function has relatively larger values around the IP, which allows for weaker 
correcting sextupoles. Having smaller sextupole strengths is crucial for not destroying the dynamic aperture.

- The local correction helps in confining the chromatic wave to within the interaction region.

This approach comes with the complication of controlling the dispersion and its derivative at the IP. This is especially true for electron beams due to the fact that using dipoles close to IR will generate bad synchrotron radiation, which must be shielded from the detector area.

For MEIC we have adopted this modern approach of correcting chromaticity both locally in the IR and using sextupoles at the arcs as well.

\section{II.6.3 Phase advance adjustment between the two $\beta$ - insertions}

A scheme to compensate the second order chromaticity was introduced in [26] for the SSC. Later it was explored as an upgrade option for LHC [27]. A brief account of the scheme and theoretical background will be given here. In the following chapter a detailed discussion about how this scheme can be adopted for the MEIC ring will be presented.

Considering a collider ring with a set of $N$ IRs, each has a set of final focusing quadrupoles $(F F)$. Let us define the integration done over the final focus quadrupoles left $(L)$ and right $(R)$ of the IP:

$$
\begin{aligned}
I_{x}^{R_{i}} & =\int_{F F_{i}} d s k(s) \beta_{x}(s) \\
I_{x}^{L_{i}} & =\int_{F F_{i}} d s k(s) \beta_{x}(s) \\
I_{y}^{R_{i}} & =-\int_{F F_{i}} d s k(s) \beta_{y}(s) \\
I_{x}^{L} R_{i} & =-\int_{F F_{i}} d s k(s) \beta_{y}(s),
\end{aligned}
$$

where $k(s)$ is the quadrupole strength.

The off-momentum $\beta$-beating, $\beta^{\prime}(s)$, is given by [27]:

$$
\frac{\beta_{x, y}^{\prime}(s)}{\beta_{x, y}(s)}=-\frac{1}{2 \sin \left(2 \pi \nu_{x, y}\right)} \sum_{i=1}^{N}\left[I_{x, y}^{R_{i}}+I_{x, y}^{L_{i}}\right] \cos \left(2\left|\mu_{x, y}^{i}-\mu_{x, y}(s)\right|-2 \pi \nu_{x, y}\right),
$$


where $\mu_{x, y}^{i}$ is the phase advance at $I P_{i}$, and $Q_{x, y}$ is the horizontal (vertical) tune of the machine. At the collision point it becomes

$$
\frac{\beta_{i}^{\prime *}}{\beta_{i}^{*}}=-\frac{1}{2 \sin (2 \pi \nu)} \sum_{j=1}^{N}\left[I^{R_{j}}+I^{L_{j}}\right] \cos \left(2\left|\mu^{i j}\right|-2 \pi \nu\right),
$$

and $\mu^{i j}=\mu^{i}-\mu^{j}$.

The second order chromaticity is given by [27]:

$$
\xi_{2}=-\frac{1}{8 \pi \sin (2 \pi \nu)} \sum_{i=1}^{N} \sum_{j=1}^{N}\left[I^{R_{i}} I^{R_{j}}+I^{L_{i}} I^{L_{j}}+2 I^{L_{i}} I^{R_{j}}\right] \cos \left(2\left|\mu^{i j}\right|-2 \pi \nu\right) .
$$

In the case of only two symmetric IPs at the collider ring, the off momentum $\beta$-beat and second order chromaticity are given by:

$$
\begin{gathered}
\frac{\beta^{\prime *}}{\beta^{*}}=-\frac{I^{R}+I^{L}}{\sin (2 \pi \nu)} \cos \left(\mu^{i j}\right) \cos \left(\mu^{i j}-2 \pi \nu\right), \\
\xi_{2}=-\frac{\left(I^{R}+I^{L}\right)^{2}}{2 \pi \sin (2 \pi \nu)} \cos \left(\mu^{i j}\right) \cos \left(\mu^{i j}-2 \pi \nu\right) .
\end{gathered}
$$

We can conclude from above that by separating the two IPs by $(2 n+1) \frac{\pi}{2}(n$ integer) in betatron phase, one can cancel out the off momentum $\beta$-beat and the second order chromaticity.

\section{II.7 TOUSCHEK LIFETIME}

The beam lifetime in an electron storage ring is limited by a number of factors: the quantum lifetime $\tau_{q}$, scattering (elastic or inelastic) of electrons by the residual gas atoms $\tau_{g}$, Touschek scattering $\tau_{\text {Touschek }}$, and the effect of trapped ions in the electron beam potential $\tau_{\text {ion }}$. The total lifetime is given by

$$
\frac{1}{\tau_{t}}=\frac{1}{\tau_{q}}+\frac{1}{\tau_{g}}+\frac{1}{\tau_{\text {Touschek }}}+\frac{1}{\tau_{\text {ion }}}
$$

The quantum lifetime is defined as the reduction in the beam lifetime due to quantum excitations. In quantum excitation, the particle's emission of a high energy synchrotron photon causes the particle to occasionally reach large oscillation amplitudes, where the particle may cross the transverse aperture or the energy acceptance.

In this section we focus on the Touschek effect and study the case for the MEIC electron figure-8 ring. The Touschek effect [28] describes particle loss within a bunch 
due to a single particle-particle collision, where the momentum transfer from the transverse into longitudinal phase space causes the scattered particle to cross the momentum aperture of the lattice. The Touschek effect is of fundamental importance for the operation of collider rings, both in terms of beam lifetime, and beam stability.

\section{II.7.1 Computing Touschek lifetime}

The Touschek lifetime is defined as the time needed for the beam intensity to decay to half its initial value. For an initial number of particles, $N_{0}$, the reduced number after time, $t$, is given by

$$
N(t)=\frac{N_{0}}{1+t / \tau}
$$

In case of a flat electron beam, $\left(\varepsilon_{x} / \beta_{x} \gg \varepsilon_{y} / \beta_{y}\right)$, the lifetime may be calculated by $[29]$

$$
\frac{1}{\tau}=\frac{N r_{0}^{2} c}{8 \pi \gamma^{3} \sigma_{s} L} \int_{L} \frac{D(\xi) d s}{\sigma_{x}(s) \sigma_{y}(s) \sigma_{x^{\prime}}(s) \delta_{a c c}^{2}(s)},
$$

where $r_{0}$ is the classical electron radius, $c$ is the speed of light, $N$ is the number of particles per bunch, $L$ is the machine path length, $\sigma_{s}$ is the rms bunch length, and $\sigma_{x}(s), \sigma_{y}(s)$ are the rms horizontal and vertical beam sizes. For horizontal bending lattices, $\sigma_{x}(s), \sigma_{y}(s)$ are given by:

$$
\begin{aligned}
\sigma_{x}(s) & =\sqrt{\varepsilon_{x} \beta_{x}(s)+\left(\sigma_{\delta_{p}} \eta_{x}(s)\right)^{2}}, \\
\sigma_{y}(s) & =\sqrt{\varepsilon_{y} \beta_{y}(s)}
\end{aligned}
$$

where $\varepsilon_{x}$ and $\varepsilon_{y}$ are the horizontal and vertical emittance respectively, $\eta_{x}, \eta_{x}^{\prime}$ are the horizontal dispersion and its derivative, $\sigma_{\delta_{p}}$ is the rms relative momentum spread, and $\sigma_{x^{\prime}}(s)$ is the rms horizontal beam divergence. The rms horizontal beam divergence for $x \approx 0$ is given by:

$$
\sigma_{x^{\prime}}(s)=\frac{\varepsilon_{x}}{\sigma_{x}(s)} \sqrt{1+\frac{H(s) \sigma_{\delta_{\mathrm{p}}}^{2}}{\varepsilon_{x}}} .
$$

The chromatic invariant and the Twiss function in $x$ are defined, respectively, as [29]:

$$
\begin{aligned}
H(s) & =\gamma_{x}(s) \eta_{x}^{2}(s)+2 \alpha_{x}(s) \eta_{x}(s) \eta_{x}^{\prime}(s)+\beta_{x}(s)\left[\eta_{x}^{\prime}(s)\right]^{2} \\
\gamma_{x}(s) & =\frac{1+\alpha_{x}^{2}(s)}{\beta_{x}(s)}
\end{aligned}
$$


with

$$
\xi(s)=\left(\frac{\delta_{a c c}(s)}{\gamma \sigma_{x^{\prime}}(s)}\right)^{2}
$$

and

$$
D(\xi)=\int_{0}^{1}\left(\frac{1}{u}-\frac{1}{2} \ln \frac{1}{u}-1\right) e^{-\xi / u} d u .
$$

As the particles scattered by Touschek scattering leave the stable RF bucket, they start coasting outside the stable bucket. A number of interest is the percentage of particles outside the RF bucket, which is given by [30]

$$
N_{\text {coast }}=\frac{t / \tau}{1+t / \tau} N_{0}
$$

A rough, order of magnitude estimate of the beam lifetime can be obtained by using effective estimation of the $\beta$ function to compute the rms transverse beam divergence, $\sigma_{x^{\prime}}=\sqrt{\varepsilon_{x} / \beta_{\text {eff }}}$, and assuming that the function $D(\xi)$ is well-estimated by a constant. A more accurate estimate of the Touschek effect is obtained by estimating the integral in Eq. (92) as a sum over all $N$ elements in the lattice:

$$
\frac{1}{\tau_{\text {Touschek }}} \approx \frac{N r_{0}^{2} c}{8 \pi \gamma^{3} \sigma_{s} L} \sum_{i=1}^{N} \frac{D(\xi) \Delta s_{i}}{\sigma_{x}\left(s_{i}\right) \sigma_{y}\left(s_{i}\right) \sigma_{x^{\prime}}\left(s_{i}\right) \delta_{a c c}^{2}\left(s_{i}\right)}
$$

where the beam parameters, such as $\alpha, \beta$ functions as well as the dispersion, $\eta$, are all assumed constant (therefore yielding constant beam sizes $\sigma_{x}, \sigma_{y}$, and divergence $\left.\sigma_{x^{\prime}}\right)$, in each of the $N$ lattice elements.

\section{II.7.2 Touschek lifetime in case of constant momentum acceptance}

We first carry out calculations for the Touschek lifetime with the constant momentum acceptance, $\delta_{a c c}$, in Eq. (99). The dependence of the Touschek lifetime on the constant momentum acceptance for the MEIC $135^{\circ}$-phase advance lattice is given in Fig. (26), and for the $90^{\circ}$ phase advance lattice in Fig. (27).

\section{II.7.3 Touschek lifetime in case of variable dynamic transverse momen- tum acceptance}

The momentum acceptance of an electron ring is limited by two factors: the height of the RF bucket, and the transverse acceptance of the lattice where particles with momentum deviations have a closed orbit which hits the vacuum chamber [29]. This 


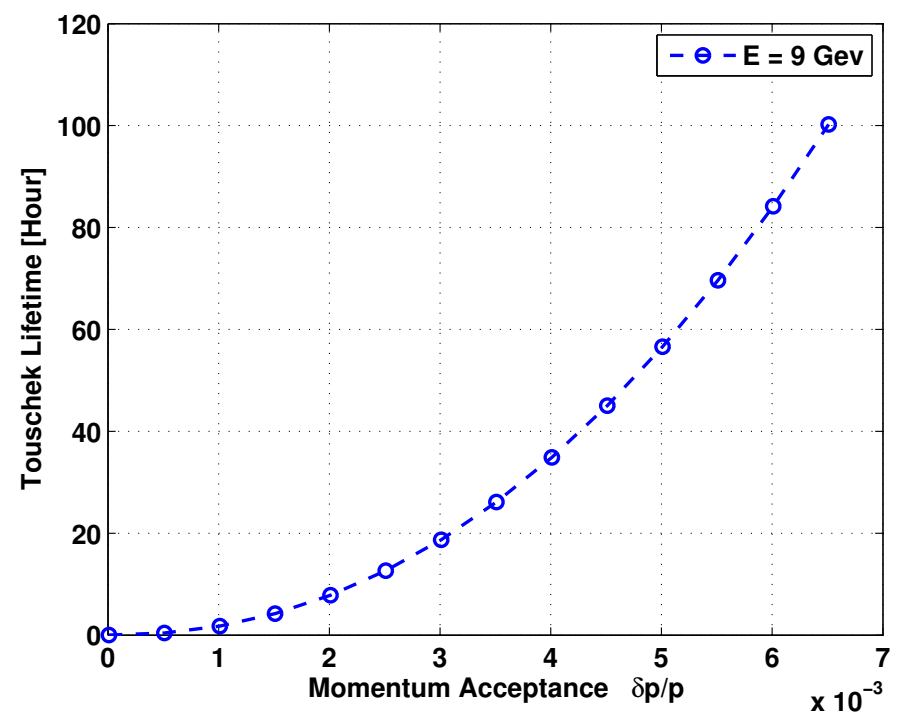

FIG. 26: Touschek lifetime for the $9 \mathrm{GeV}$ MEIC $135^{\circ}$ lattice.

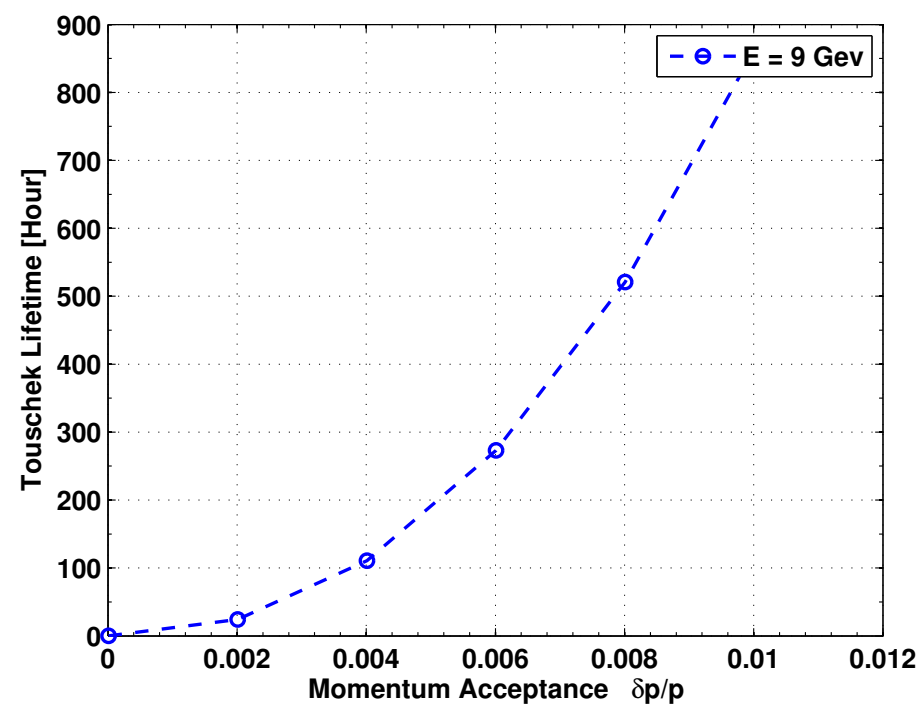

FIG. 27: Touschek lifetime for the $9 \mathrm{GeV}$ MEIC $90^{\circ}$ lattice. 
momentum acceptance is provided by the lattice and varies throughout the ring. In the case of a linear lattice, it is given by [29]

$$
\delta_{a c c}\left(s_{0}\right)= \pm \min \left(\frac{a_{x}(s)}{\sqrt{H\left(s_{0}\right) \beta_{x}(s)}+|\eta(s)|}\right) \text {, }
$$

with the scattering event taking place at $s=s_{0} . a_{x}(s)$ is the physical aperture (beam pipe half width) at location $s$. In the case of a lattice with high nonlinearities, tracking is needed to determine the transverse acceptance of the lattice.

We estimate the lower limit of the Touschek lifetime by using the minimum beam pipe half width size along the beamline in Eq. (100), i.e., $a_{x}(s)=a_{x, \min }=$ const. The results are shown in Fig. (28) and Fig. (29). For the minimum Touschek lifetime of 2 hours, the minimum beam pipe half width of $a_{x, \text { min }} \gtrsim 10 \mathrm{~mm}$ is required for the $135^{\circ}$ lattice, while much less than this is required for the $90^{\circ}$ lattice.



FIG. 28: Touschek lifetime for the $9 \mathrm{GeV}$ MEIC $135^{\circ}$ lattice as a function of the minimum size of the beam pipe half width. 


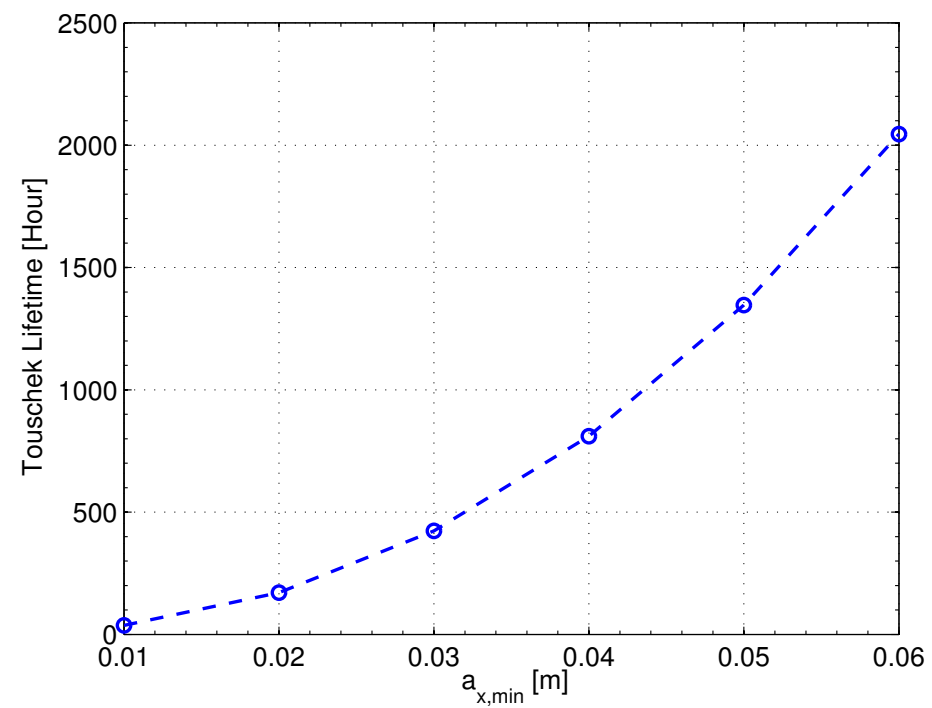

FIG. 29: Touschek lifetime for the $9 \mathrm{GeV}$ MEIC $90^{\circ}$ lattice as a function of the minimum size of the beam pipe half width. 


\section{II.8 BEAM-BEAM TUNE SHIFT}

Beam-beam interactions cause an emittance growth of colliding beams, which leads to reduction of collider luminosity. MEIC design parameters with very small $\beta^{*}$ at the IP, and moderate crab crossing angle (50 to $100 \mathrm{mrad}$ ) require an investigation of the beam-beam effect. A recent simulation study of the beam-beam effect [31] was carried out for the MEIC with a $5 \mathrm{GeV}$ electron beam colliding with a $60 \mathrm{GeV}$ proton beam. The betatron tune working point, which performs at the designed luminosity was found. The study demonstrated that the proposed parameter set is safely separated from beam-beam instabilities and preserves the design luminosity.

Fig. (30) shows the tune footprint of 400 random orbits of both electron and proton beams. The tune spread of the proton beam is smaller than the spread of the electron beam. The tune footprint of each beam is safely away from lower order resonances, which allows beams to maintain high luminosity for a very long time.

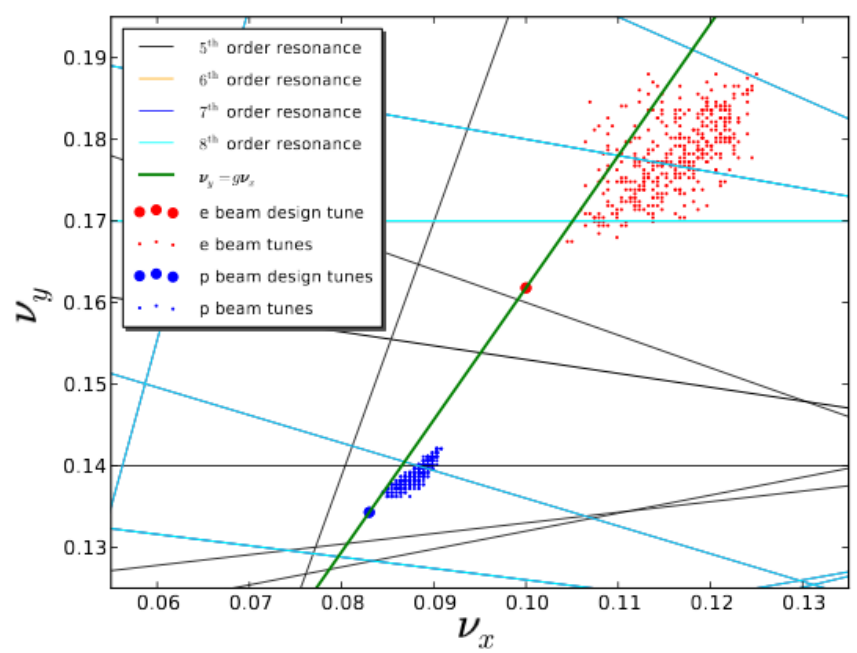

FIG. 30: Tune space for the electron beam. High order resonant lines are shown in various colors. The line for which the ratio is equal to the golden mean is shown in green. Small red and blue dots represent the tunes of the 400 random orbits from the electron and proton beams, respectively. Large red and blue dots represent design tunes for electron and proton beams. 


\section{II.9 RADIATION POWER}

The synchrotron radiation power for high energy particle beams (like the MEIC electron figure-8 ring) limits the maximum energy achievable in circular accelerator. The radiation power of protons is much smaller than those of electrons by a factor of $1.1367 \times 10^{13}$. In case of isomagnetic lattice (all bending magnets have the same bending radius) the total synchrotron radiation power is given by Eq. (101).

$$
<P_{s}[M W]>_{i s o}=0.088463 \frac{E^{4}[G e V]}{\rho[m]} I[A]
$$

Fig. (31) shows synchrotron radiation power per unit beam current as a function of bending radius for isomagnetic lattice. For an electron ring at $9 \mathrm{GeV}$ and an isomagnetic lattice with radius of curvature of $116 \mathrm{~m}$, the total radiation power is $5 \mathrm{MW} / \mathrm{A}$, while for a lattice with radius of curvature of $25 \mathrm{~m}$, the total radiation power is $23 \mathrm{MW} / \mathrm{A}$.

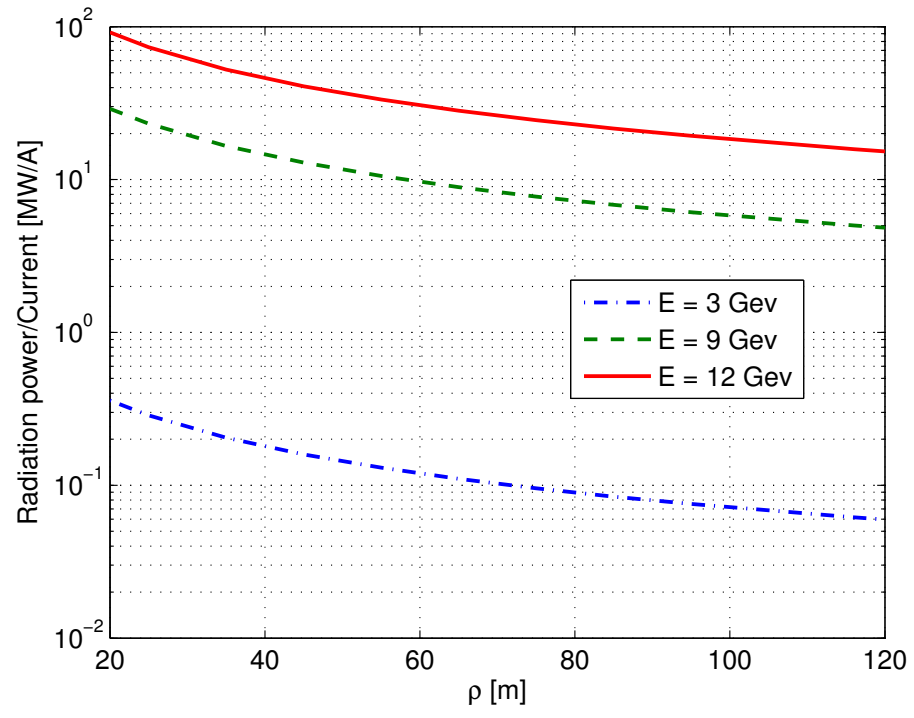

FIG. 31: Synchrotron radiation power per unit beam current as a function of bending radius at three different electron energies. The strong dependence of radiation power on the electron energy causes substantial limitations on the maximum achievable energy in circular accelerators. 


\section{II.10 SUMMARY}

The linear and non-linear equations of motion were derived from the Hamiltonian of a charged particle in electromagnetic field. Accurate expansions for the first and second order tune chromaticity were derived from the equations of non-linear motion. An analytic calculation model was developed for the tune chromaticity generated by final focus quadrupoles in a straight section. A correction mechanism using sextupole and octupole magnets was discussed. A review of chromaticity correction schemes was presented as well. Three chromaticity correction schemes of interest were evaluated and used in this thesis. The three schemes are the global chromaticity correction, local chromaticity correction, and adjusting phase advance between low $\beta$ insertions to cancel second order chromaticity. The effect of limited momentum acceptance due to chromaticity on the Touschek lifetime was studied and quantified for the considered lattice designs. An efficient code for computing Touschek lifetime for an electron flat beam was developed and used for the computations. 


\section{CHAPTER III}

\section{CHROMATICITY CORRECTION FOR MEIC}

\section{III.1 INTRODUCTION}

This chapter will discuss a preferred scheme to correct for MEIC chromaticity, beginning by discussing the natural chromaticity generated by the arc's quadrupoles and then present a comparison between two different arc lattices in correcting such chromaticty. The rest of the chapter will be dedicated to the interaction region designs and local chromatic correction. Two different IR designs will be presented. The first has non-vanishing dispersion derivative across the IP, the second IR design has a symmetric dispersion with the IP as the symmetry point; in this design both dispersion and its derivative will vanish at the IP.

\section{III.2 NATURAL CHROMATICITY FROM THE ARC SECTIONS}

This section is dedicated to a discussion of the natural chromaticity generated from the arc's quarupoles. It will explore two different linear lattice designs for the arcs, and consider the contribution of each arc lattice to the over all chromaticity of the figure- 8 ring. The motivation in this section is to evaluate different lattices choices for the figure- 8 collider ring in terms of chromaticity.

\section{III.2.1 Arc lattice with $135^{\circ}$ phase advance cells}

In this lattice $135^{\circ}$ phase advance FODO cell has been chosen so that it's close to the theoretical minimum emittance for all FODO cells. It is quite common to use such high betatron phase advance lattice for light sources, or a damping storage ring. The case of a collider ring is more challenging, as the low $\beta$-insertion is the major contributor to chromaticity. The final focus quadrupole would produce tune chromaticity comparable to the contribution of all other quadrupoles in the ring or more, and has an impact on the $\beta$ function at IP $\left(\beta^{*}\right)$ and the magnet free distance from IP to first final focus quadrupole.

The following treatment will address the natural chromaticity of a figure- 8 ring with no final focus quadrupoles, as an aid to assist in choosing the best figure- 8 cell design for the MEIC case. 
The scheme for correcting chromaticity would be solely global, where a set of interleaved sextupole families are placed in the arc cells and are used for chromatic correction. Each sextupole was placed adjacent to a quadrupole they correct. Sextupoles were added in families, every family member was placed $3 \pi$ in betatron phase from each other to cancel second order aberrations. Fig. (32) shows the sextupole magnets in arc cells.

The basic structure of the sextupole families can be put as follows:

Two sextupoles per cell: One for vertical correction next to defocusing quadrupole, and the second for horizontal correction next to focusing quadrupole.

Four families of sextupoles repeated every 4 cells: Each cell has $135^{\circ}$ phase advance, so the total phase advance between each family member is set to $3 \pi$.

Four different knobs: Enables optimizing for four different chromatic quantities (first order chromaticity, second order chromaticity, $W$ chromatic function at the IP, $W$ chromatic function at the end of the IR).

Sextupoles at the second arc: The second arc was designed to be almost identical (opposite bending) to the first arc, but the dispersion function has the opposite sign. So the same correcting sextupoles were used but with opposite polarity.

The basic structure of the first arc cells is given as following,

$$
\begin{aligned}
& \text { QD - SXTFA1 - BENDIN - QF - SXTDA1 - BENDIN } \\
& \text { QD - SXTFB1 - BENDIN - QF - SXTDB1 - BENDIN } \\
& \text { QD - SXTFC1 - BENDIN - QF - SXTDC1 - BENDIN } \\
& \text { QD - SXTFD1 - BENDIN - QF - SXTDD1 - BENDIN }
\end{aligned}
$$

where $\mathrm{QF}(\mathrm{QD})$ is the focusing (defocusing) quadrupole, BENDIN is the bending magnet in the first arc, and $\operatorname{SXT}(\mathrm{A}, \mathrm{B}, \mathrm{C}, \mathrm{D}) 1$ are sextupole magnets. The basic structure of the second arc cells is given by:

$$
\begin{aligned}
& \text { QD - SXTFA2 - BENDOUT - QF - SXTDA2 - BENDOUT } \\
& \text { QD - SXTFB2 - BENDOUT - QF - SXTDB2 - BENDOUT } \\
& \text { QD - SXTFC2 - BENDOUT - QF - SXTDC2 - BENDOUT } \\
& \text { QD - SXTFD2 - BENDOUT - QF - SXTDD2 - BENDOUT }
\end{aligned}
$$


BENDOUT is the bending magnet in the second arc with opposite polarity, and SXT(A,B,C,D)2 are sextupole magnets with exact strength and opposite polarity of those in the first arc. This structure is repeated for 7 times in each arc, with an $-I$ transformation between every sextupole and its symmetric partner in the family.

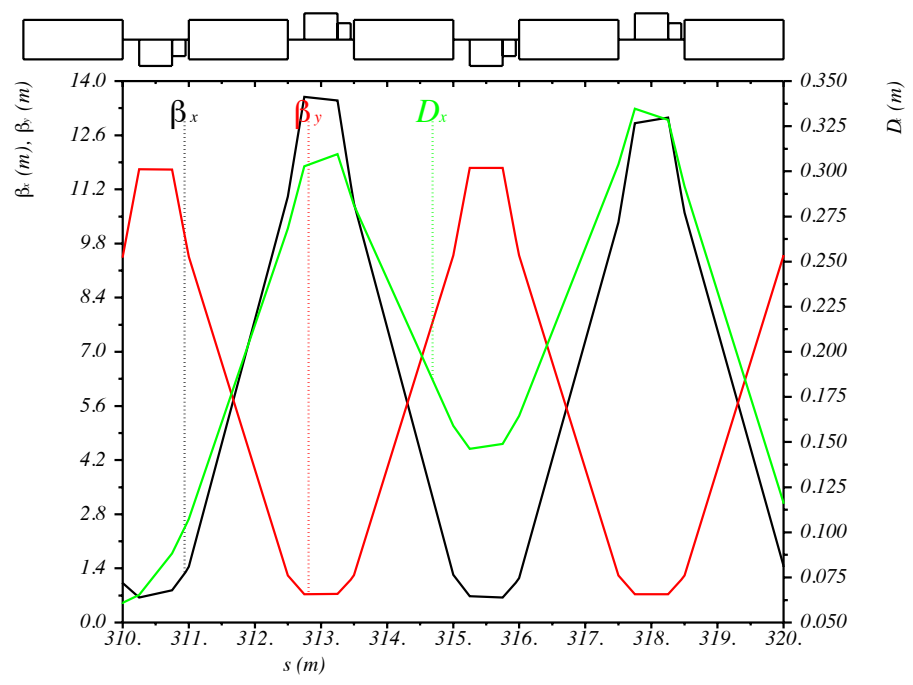

FIG. 32: MEIC arc cells with sextupole magnets (smallest boxes). Sextupoles are placed at locations with good aspect ratios between $\beta_{x}$ and $\beta_{y}$.

The chromaticity correction was carried out using the computer code (MAD-X). During the optimization process, the arc sextupoles were optimized to correct the first and the second order tune chromaticity. Fig. (33) shows the tune variation with momentum deviation after correction in both horizontal and vertical planes. The momentum acceptance corresponds to more than 115 hours of Touschek lifetime.

A track was launched using the MAD-X PTC tracking module for a figure- 8 ring with all sextupoles on. The dynamic aperture was found to be more than sufficient; $85 \sigma$ in both transverse dimensions. The limiting factor for dynamic aperture is the arc's correcting sextupoles. 


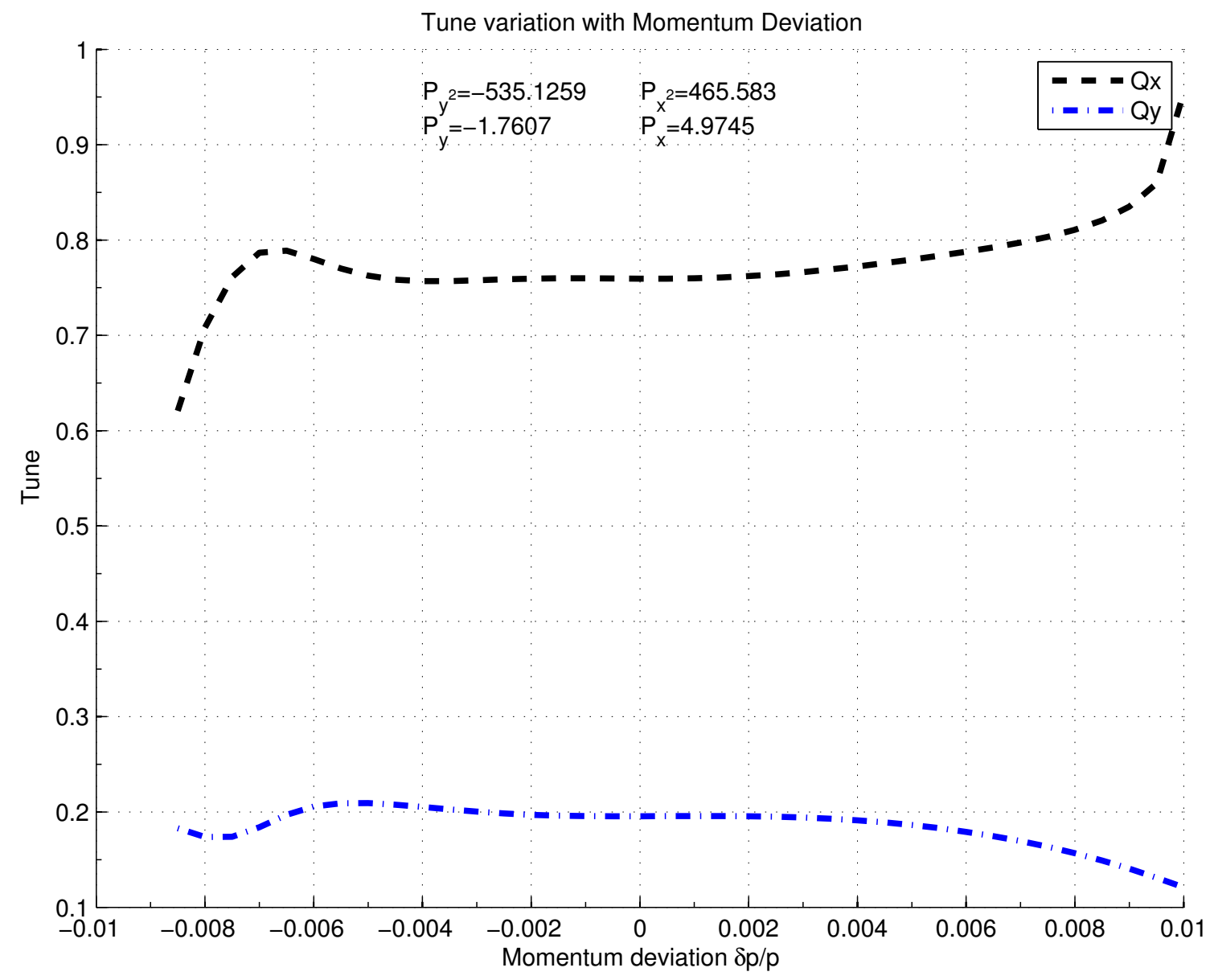

FIG. 33: Tune variation with momentum deviation for the MEIC figure-8 ring without the interaction region. Momentum acceptance is $\pm 0.8 \%$. 


\section{III.2.2 Arc lattice with $90^{\circ}$ phase advance cells}

The $90^{\circ}$ phase advance per cell is a result of weaker focusing quadrupoles. Lesser quadrupole strengths would lead to smaller natural chromaticity and therefore weaker correcting sextupoles. This would also make a larger dynamic aperture. This scheme for correcting chromaticity is solely global, just as before. Interleaved sextupole families at the arcs were used for chromatic correction. Each sextupole was placed in the arc cells adjacent to a quadrupole. Every sextupole family member was placed at $\pi$ phase from each other to cancel second order aberrations. Fig. (34) shows sextupole magnets in arc cells. The basic structure of sextupole families can be put as follows:

Number of sextupoles per cell: One sextupole every two cells for vertical correction next to the defocusing quadrupole (non interleaved sextupole families), and one sextupole for horizontal correction next to the focusing quadrupole (interleaved sextupole families).

Two families of sextupoles repeated every 2 cells: Each cell has $90^{\circ}$ phase advance so the total phase advance between each family member is $\pi$.

Two different knobs: Enables optimizing for two different chromatic quantities (first order chromaticity, second order chromaticity). In this case more knobs need to be created at the local correction block to correct Montague chromatic functions $W$.

Sextupoles at the second arc: The second arc was designed to be almost identical (opposite bending) to the first arc, but the dispersion function has the opposite sign. So the same correcting sextupoles were used but with opposite polarity.

The basic structure of the first arc cells is given as following,

$$
\begin{gathered}
\text { CELLRA }=\text { QF - SF1A - DRIFT - QD - BNDIN - QD2 } \\
\text { CELLRB = QF - SF1B - DRIFT - QD - BNDIN - QD2 } \\
\text { CELLR = CELLRA - SD1A - RCELLRB - CELLRA - SD1A - } \\
\text { RCELLRB }
\end{gathered}
$$

where $\mathrm{QD}(\mathrm{QF})$ is the focusing (defocusing) quadrupole, BNDIN is the bending magnet in the first arc, and $\mathrm{SD}(\mathrm{SF})(\mathrm{A}, \mathrm{B})$ are sextupole magnets, and the RCELLRB means is the reverse lattice of CELLRB. 
The lattice structure at the second arc is given by:

\section{CELLLA = QF - SF2A - DRIFT - QD - BNDOUT - QD2 \\ CELLLB = QF - SF2B - DRIFT - QD - BNDOUT - QD2 \\ CELLL = CELLLA - SD2A - RCELLLB - CELLLA - SD2A - RCELLLB}

This structure is repeated 29 times in each arc, with an $-I$ transformation between every sextupole and its symmetric partner in the family.



FIG. 34: MEIC arc cells with sextupole magnets (smallest boxes). Sextupoles are placed at locations with good aspect ratio between $\beta_{x}$ and $\beta_{y}$.

The correction was studied using the computer code (MAD-X). During the optimization process the sextupole knobs in the arcs were optimized to correct first and second order tune chromaticity. Fig. (35) show tune variation with momentum deviation after correction in both horizontal and vertical planes.

Tracking was carried out using the MAD-X PTC tracking module for a figure8 ring with all sextupoles on. The dynamic aperture was found to be more than sufficient at $120 \sigma$ in both transverse dimensions. Clearly, this lattice has a larger dynamic aperture than the $135^{\circ}$ case. 


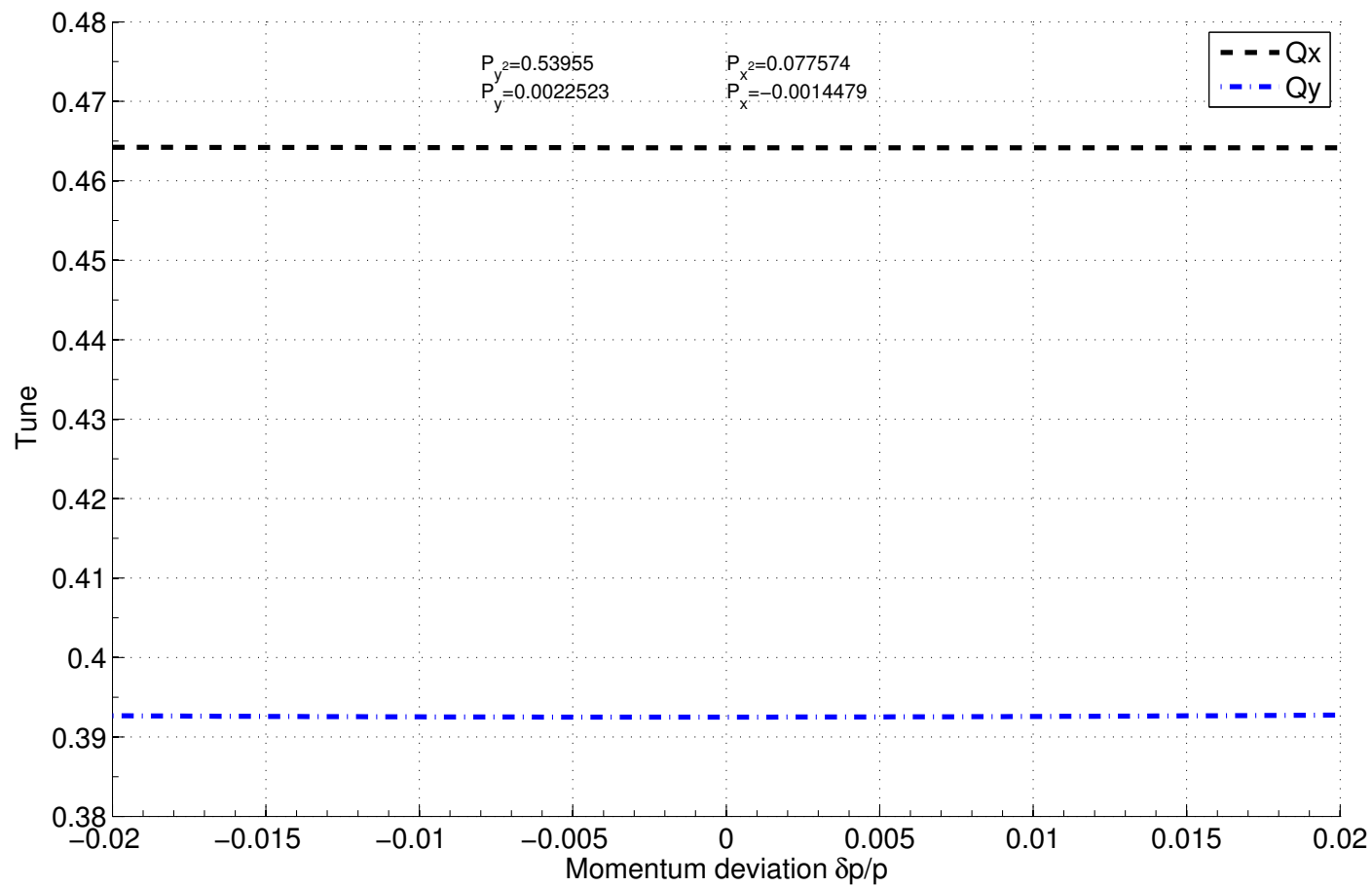

FIG. 35: Tune variation with momentum deviation for MEIC figure-8 ring without the interaction region. The momentum acceptance is $\pm 2.0 \%$. 


\section{III.3 ANALYSIS OF CHROMATICITY OF THE FINAL FOCUSING QUADRUPOLES}

The final focus quadrupoles provide the major contribution to chromaticity. Referring to Eq. (49), the first order chromaticity from quadrupole contribution is given by

$$
\begin{aligned}
\xi_{x} & =-\frac{1}{4 \pi} \oint \beta_{x} b_{2}(s) d s, \\
\xi_{y} & =\frac{1}{4 \pi} \oint \beta_{y} b_{2}(s) d s .
\end{aligned}
$$

The contribution to the first order chromaticity due to a quadrupole magnet increases with the $\beta$ function and the quadrupole strength. One realizes that in order to optimize for the lowest possible contribution to chromaticity from a final focus quadrupole, we must limit the maximum $\beta$ function at that location while maintaining the design requirements of $\beta^{*}$ and magnet free space from the IP to the first final focusing quadrupole.

The betatron function can be given in terms of its value at the IP by

$$
\beta(s)=\beta^{*}+\frac{s^{2}}{\beta^{*}},
$$

where $s$ is the distance from the interaction point. As in the MEIC design, the magnet free distance, $L$, must be large enough to enable the installation of large particle detectors. As a result the $\beta$ function will increase dramatically at the final focus quadrupoles, contributing to the chromaticity.

A rough estimate of the contribution of the final focus quadrupoles to chromaticity using Eq. (102) was calculated. The results are given in Table (10) for a non-vanishing dispersion derivative at the IP case and Table (11) for a vanishing dispersion derivative at the IP. The total chromaticity is the sum of the chromatic contributions of every quadrupole in the lattice.

\section{III.4 CORRECTING CHROMATICITY FOR THE MEIC}

In this section, the general correction scheme adopted for the MEIC will be discussed and the correction scheme will be described in more detail. The final results after correction will be presented in addition to dynamic aperture tracking.

There are two main goals for correcting chromaticity. The first is to correct the tune chromaticity to enable stability of the beam dynamics and a long Touschek 
TABLE 10: Estimates of the first order chromaticity from the MEIC final focus quadrupoles with non-vanishing dispersion derivative at the interaction point.

\begin{tabular}{lcccc}
\hline \hline Magnet & $K\left[\mathrm{~m}^{-2}\right]$ & $L[\mathrm{~m}]$ & $\beta_{x}\left(\beta_{y}\right)[\mathrm{m}]$ & $\xi_{x}\left(\xi_{y}\right)$ \\
\hline Horizontal Quad & 1.79 & 0.5 & $210(324)$ & $-188(289)$ \\
Vertical Quad & -1.71 & 0.5 & $122(612)$ & $104(-523)$ \\
\hline \hline
\end{tabular}

TABLE 11: Estimates of the first order chromaticity from the MEIC final focus quadrupoles with vanishing dispersion derivative at the interaction point.

\begin{tabular}{lcccc}
\hline \hline Magnet & $K\left[\mathrm{~m}^{-2}\right]$ & $L[\mathrm{~m}]$ & $\beta_{x}\left(\beta_{y}\right)[\mathrm{m}]$ & $\xi_{x}\left(\xi_{y}\right)$ \\
\hline Horizontal Quad & 2.70 & 0.5 & $281(320)$ & $-397(432)$ \\
Vertical Quad & -3.0 & 0.5 & $109(539)$ & $163(-808)$ \\
\hline \hline
\end{tabular}

lifetime. The second goal is to reduce the off-momentum betatron beats ( $\beta$ chromaticity) at the IP to achieve the required beam size at the IP, which produces the design luminosity of the collider.

\section{III.4.1 Anti-symmetric dispersion around the interaction point}

In the following sections. A scheme for correcting chromaticity for an interaction region with an anti-symmetric dispersion across the interaction point will be presented, in this case only the dispersion vanishes at the interaction point while its derivative has a finite value. The interaction region linear lattice was presented in detail in section (I.4.3). In this part the local and global correction scheme of the first and second order chromaticity will be presented.

Local correction This correction was done using a set of sextupoles placed symmetrically around the IP in places with suitably large values of dispersion and betatron function, in order to reduce the sextupole strength needed for the correction. The dispersion required for the chromatic correction was generated from the arc dipoles and left to propagate through the IR while maintaining dispersion-free IPs 




FIG. 36: Twiss function near the IR with a non-vanishing dispersion derivative at the IP.

with an antisymmetric dispersion wave around each of the two IPs. Montague chromatic functions, $W_{x, y}$, were used as a measure of $\beta$ chromaticity at the IP. Local correcting sextupoles around the IP were used to reduce those functions from $10^{3}$ to $10^{-4}$, while confining them to acceptable values for the rest of the ring. Six sextupole pairs were used in this process; the pair closest to the IP was used to eliminate the $W_{x, y}$ functions at the IP, and the remaining five pairs were invoked to confine chromatic functions within the IR. Fig. (37) shows Montague functions locally around the IP after correction. Second order chromaticity arising from the IR's final focus quadrupoles and correcting sextupoles was mitigated by adjusting the phase advance between the two symmetric interaction regions to be $\pi(1 / 2+n)$ (where $n$ is an integer number). 


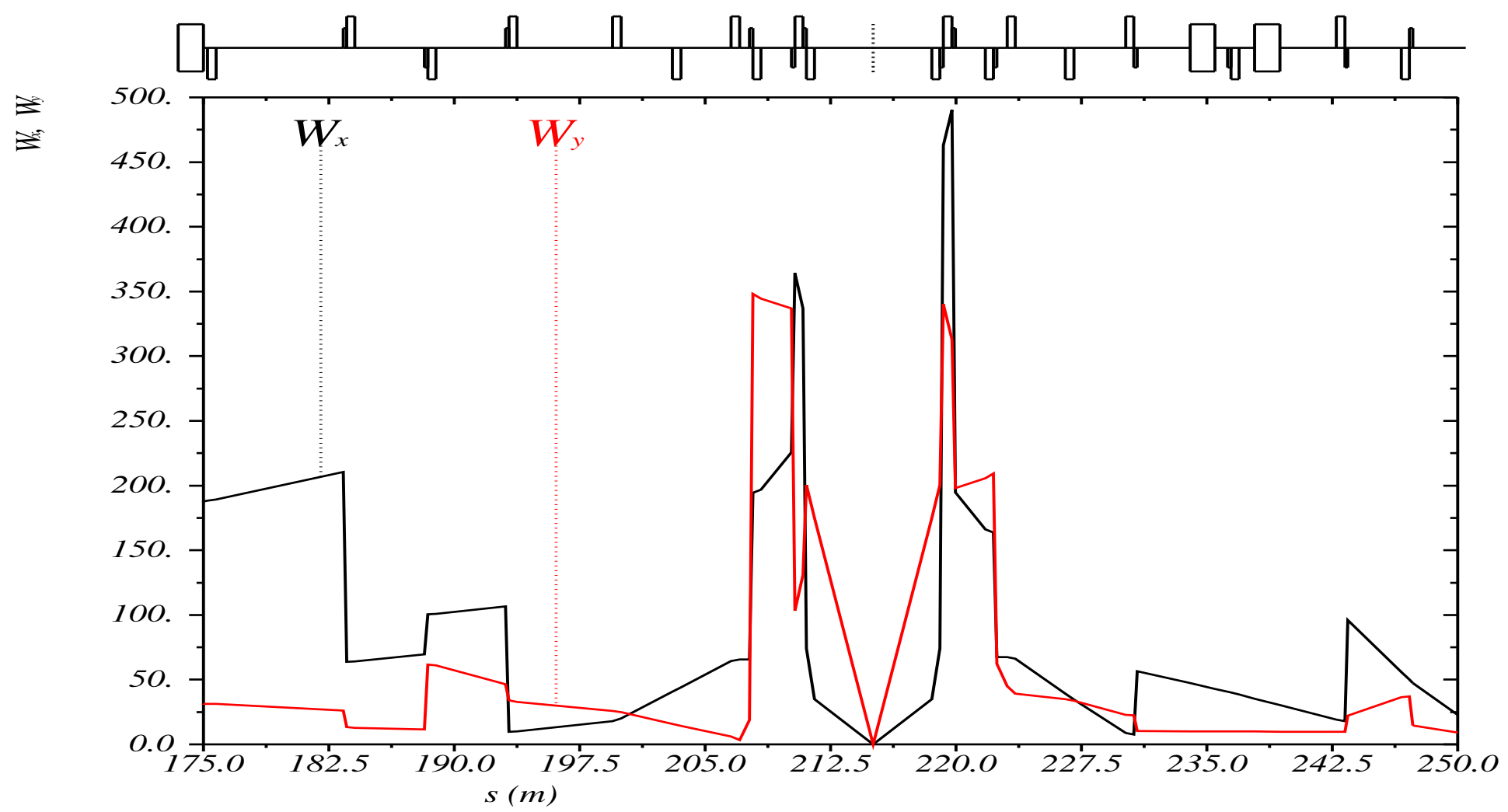

FIG. 37: MEIC Montague chromatic functions at the IP after correction with local sextupoles. Sextupoles are placed at locations with a good aspect ratio between $\beta_{x}$ and $\beta_{y}$. 


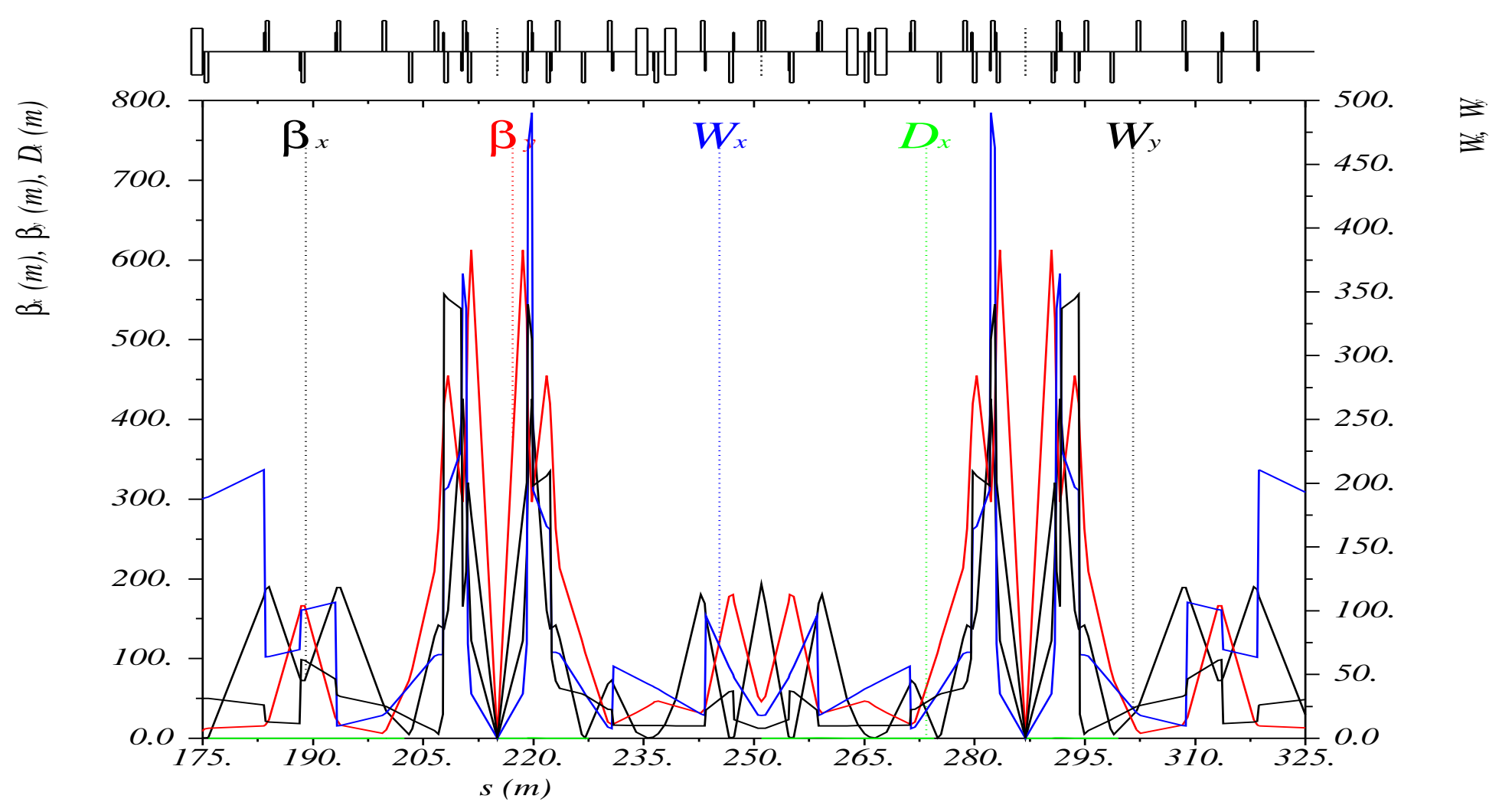

FIG. 38: MEIC Montague chromatic functions in the two interaction regions after correction with local sextupoles. 


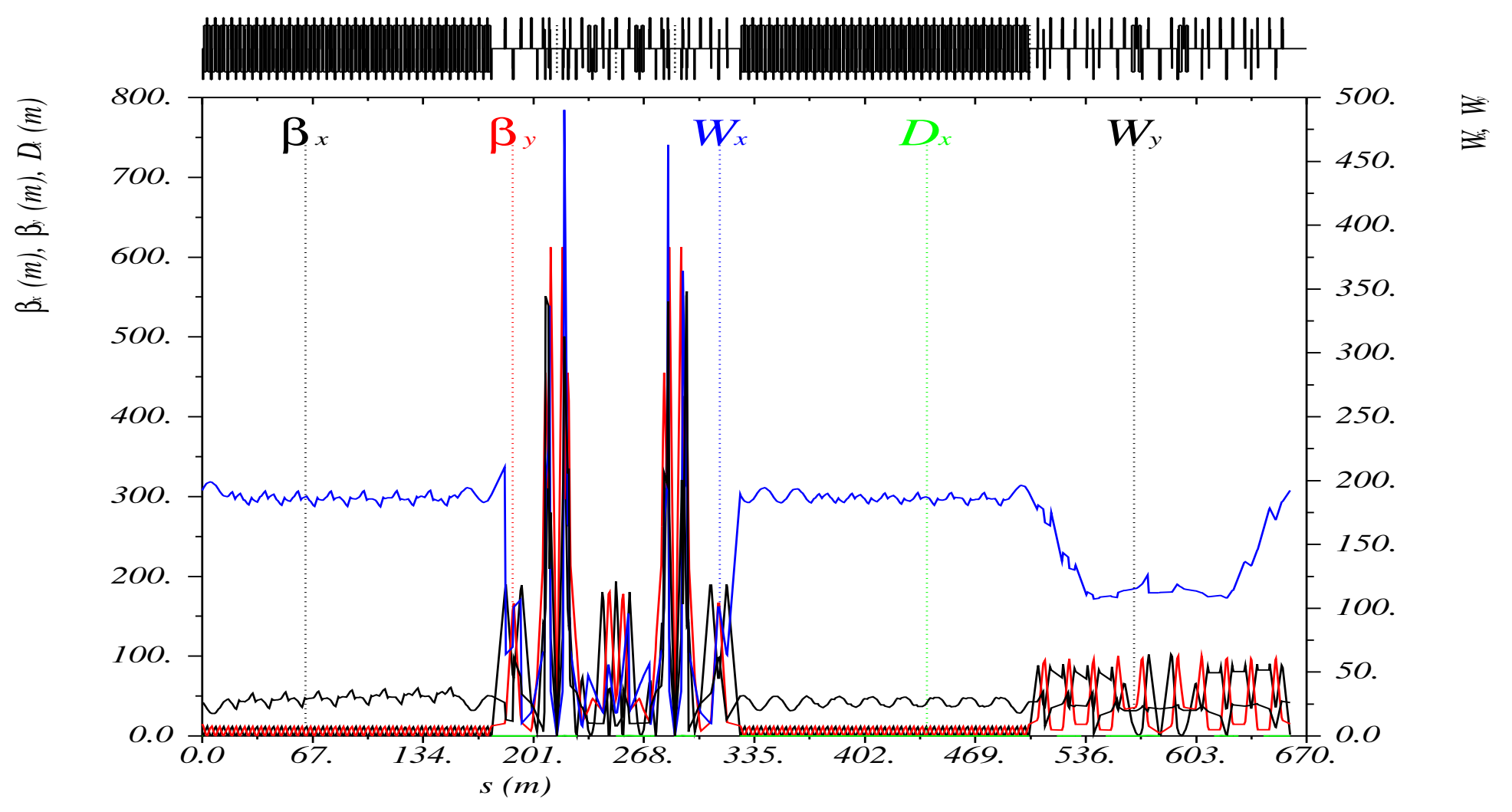

FIG. 39: MEIC Montague chromatic functions in the figure- 8 ring after sextupole correction. 
Global correction The residual chromaticity propagated from the IR, in addition to the natural chromaticity generated by the arc quadrupoles, was compensated with families of sextupoles placed in the arcs and in the other straight which has no IP. In the arc case, sets of interleaved sextupole families were used, every family member was placed at $3 \pi$ phase from each other to cancel second order effects. The main goal of the sextupole families in the arcs is to reduce the tune variation with momentum deviation (tune chromaticity). A global optimization was used to reduce it. Fig. (40) shows tune variation with momentum offset after the correction.
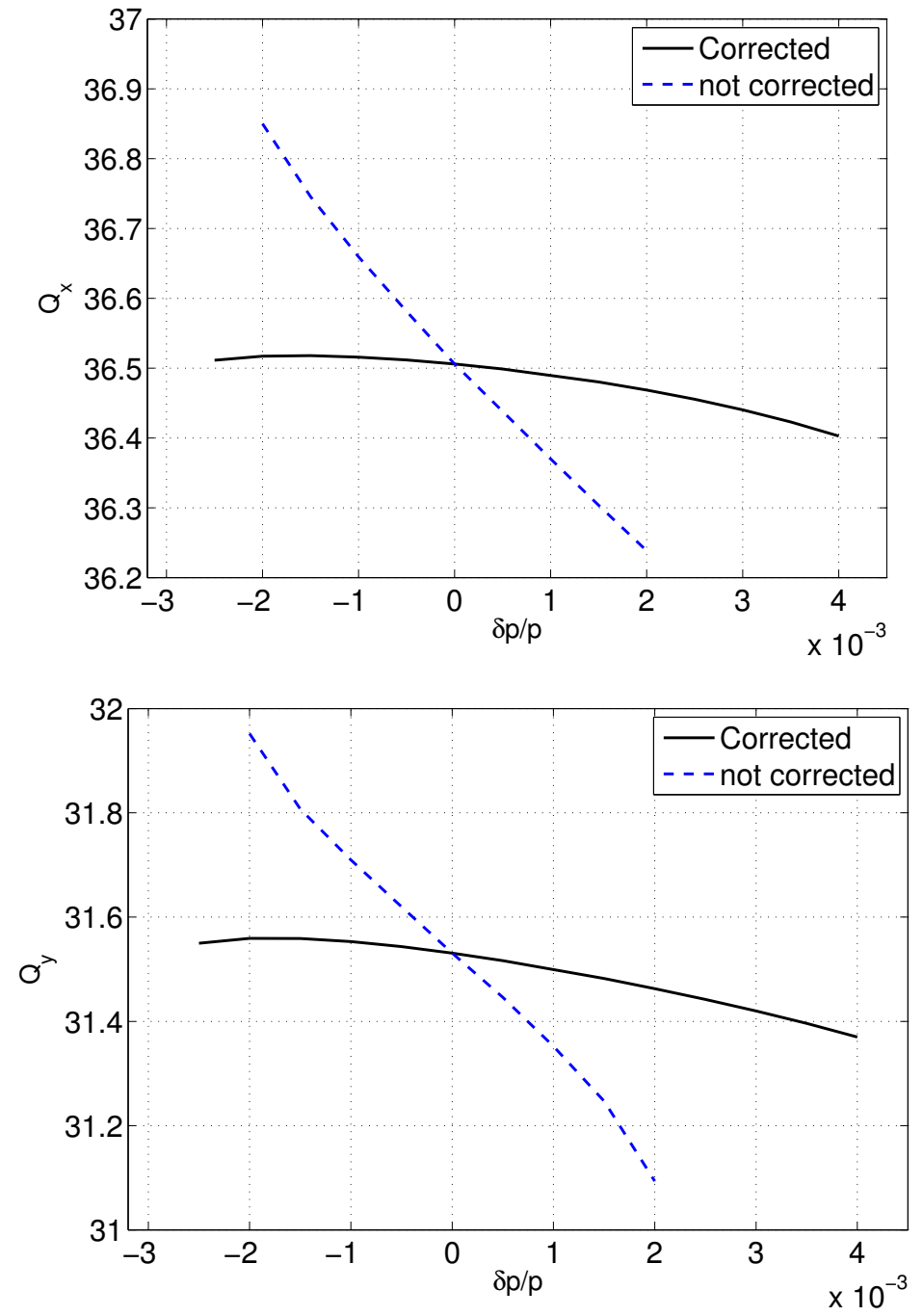

FIG. 40: MEIC tune variation with momentum deviation before and after correction. Momentum acceptance is $\pm 0.3 \%$. 
A Touschek lifetime calculation showed that for the lattice under study one needs at least 0.003 momentum acceptance for the lifetime to reach 3 hours. Fig. (26) shows the Touschek lifetime dependance on momentum acceptance. The MEIC is expected to have shorter depolarization time scale, hence the electron ring will be refilled before Touschek effect becomes an issue. A dynamic aperture study showed that the unbalanced local correcting sextupoles reduced the dynamic aperture. The anitsymmteric dispersion across the IP made it mandatory for sextupoles of the same family to have opposite signs and the second order aberrations were not canceled. 


\section{III.4.2 Symmetric dispersion around the interaction point}

In the following section, a scheme for correcting chromaticity for an interaction region with a symmetric dispersion across the interaction point is presented. In this case both dispersion and its derivative vanish at the interaction point. The interaction region linear lattice was presented in detail in Section I.4.3. In this part, we will present the linear lattice design of the interaction region matching section and adjacent chromaticity correction block. The last part of this section will be dedicated to local and global correction schemes of the first and second order chromaticity.

\section{Interaction region matching section}

A matching section was designed to match the large betatron functions and dispersion to the periodic structure of the chromatic correction block. The lattice structure of the matching section is given as follows:

[DRIFT, OIRD2, SIRD1, QFFMTCH04, DRIFT, OIRF2, SIRF1, QFFMTCH03, DFF, QFFMTCH02,OIRD1, SIRD2, DRIFT, QFFMTCH01, DRIFT,OIRF1, SIRF2]

The following table defines each lattice element and its parameters:

TABLE 12: Magnets of the interaction region matching section.

\begin{tabular}{llcccc}
\hline \hline Element & Type & $L[\mathrm{~m}]$ & $K\left[\mathrm{~m}^{-2}\right]$ & $M\left[\mathrm{~m}^{-3}\right]$ & $O\left[\mathrm{~m}^{-4}\right]$ \\
\hline QFFMTCH01 & Quad. & 0.5 & 0.79 & 0 & 0 \\
QFFMTCH02 & Quad. & 0.5 & -1.20 & 0 & 0 \\
QFFMTCH03 & Quad. & 0.5 & 1.130 & 0 & 0 \\
QFFMTCH04 & Quad. & 0.5 & -1.11 & 0 & 0 \\
SIRF1 & Sext. & 0.2 & 0 & 14.2 & 0 \\
SIRF2 & Sext. & 0.2 & 0 & -960 & 0 \\
SIRD1 & Sext. & 0.2 & 0 & -244 & 0 \\
SIRD2 & Sext. & 0.2 & 0 & 147 & 0 \\
OIRD1 & Oct. & 0.2 & 0 & 0 & 37366 \\
OIRD2 & Oct. & 0.2 & 0 & 0 & 53865 \\
OIRF1 & Oct. & 0.2 & 0 & 0 & 1232930 \\
OIRF2 & Oct. & 0.2 & 0 & 0 & -4960 \\
\hline \hline
\end{tabular}




\section{Description of the chromaticity correction block}

The chromaticity correction block was designed to serve two main purposes. The first is to generate the Twiss function suitable for chromaticity correction ( $\beta$ functions with a reasonable aspect ratio between the horizontal and vertical peaks, $180^{\circ}$ degrees in phase advance in both planes at sextupole locations, and a dispersion function which has the same sign at all members of each sextupole family). The second goal of the chromatic correction block is to mitigate $\beta$ function to adequate values that are matched to the arc using a matching section. Fig. (41) shows the Twiss parameters through the IR and the chromaticity correction block.

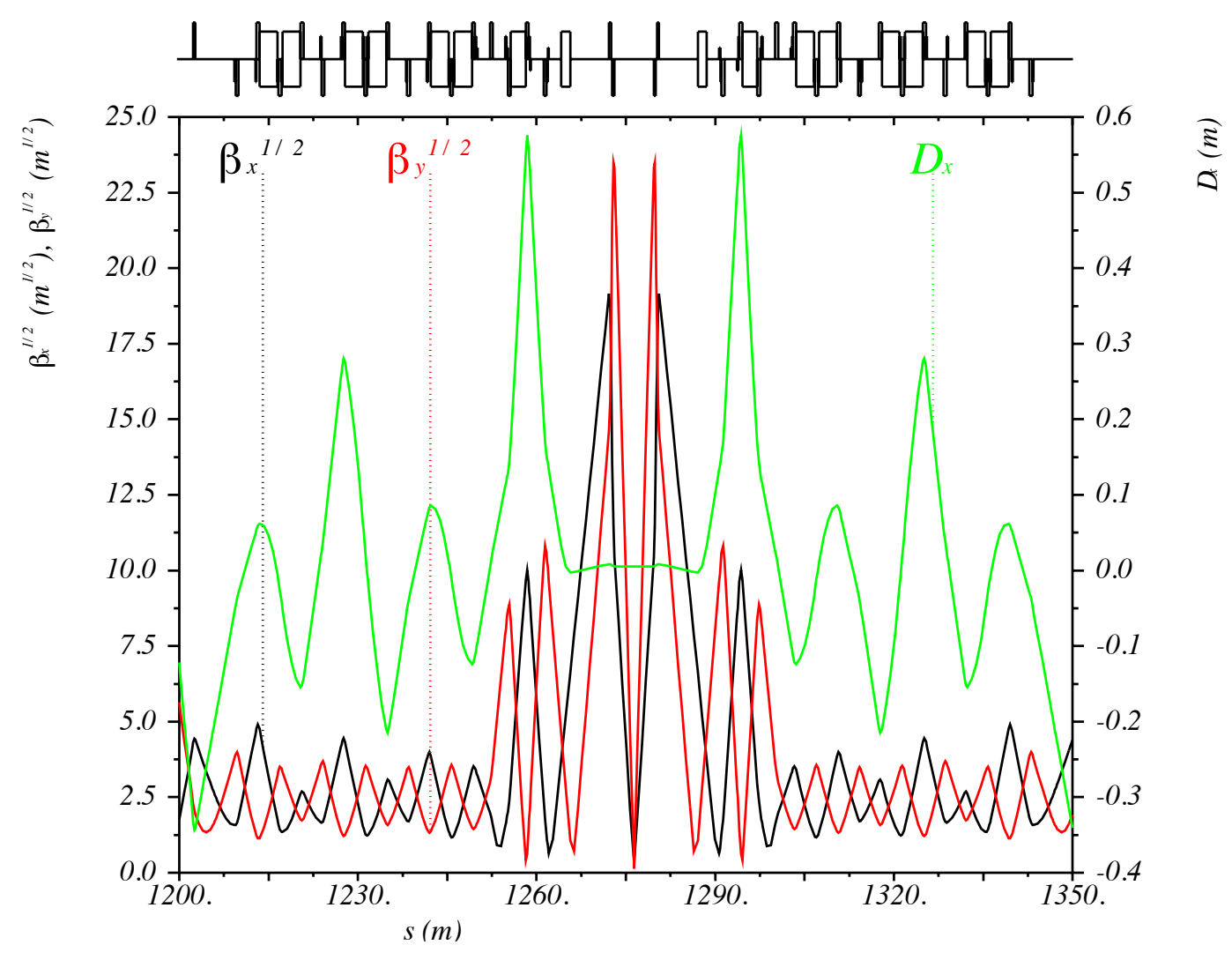

FIG. 41: Twiss functions at the chromaticity correction block.

The lattice structure of the block can be listed as follows:

[DRIFT, SIRD4, QCCB02, DRIFT, SIRF3, QCCB01, DFF2, SIRD3, QCCB02, SIRD3, DFF1, QCCB01] 
[DRIFT, SIRD6, QCCB02, DRIFT, SIRF4, QCCB01, DFF2, SIRD5, QCCB02, SIRD5, DFF1, QCCB01]

[DRIFT, SIRD4, QCCB02, DRIFT, SIRF3, QCCB01, DFF2, SIRD3, QCCB02, SIRD3, DFF1, QCCB01]

where QCCB01(QCCB02) are the focusing (defocusing) quadrupole, DFF1 and DFF2 are bending magnets used to mitigate dispersion and match it to the dispersion in the arcs, and $\mathrm{SD}(\mathrm{SF})(\mathrm{A}, \mathrm{B})$ are sextupole magnets. Magnet parameters are given in Table (13).

TABLE 13: Magnets of the chromaticity correction block.

\begin{tabular}{lccccc}
\hline \hline Element & Type & $L[\mathrm{~m}]$ & $\theta[\mathrm{rad}]$ & $K\left[\mathrm{~m}^{-2}\right]$ & $M\left[\mathrm{~m}^{-3}\right]$ \\
\hline DFF1 & Dipole & 3.0 & 0.05 & 0 & 0 \\
DFF2 & Dipole & 3.0 & -0.05 & 0 & 0 \\
QCCB01 & Quad & 0.5 & 0 & 0.8497 & 0 \\
QCCB02 & Quad & 0.5 & 0 & -0.8497 & 0 \\
SIRF3 & Sext & 0.2 & 0 & 0 & -244.5 \\
SIRF4 & Sext & 0.2 & 0 & 0 & -244.5 \\
SIRD3 & Sext & 0.2 & 0 & 0 & -10.209 \\
SIRD4 & Sext & 0.2 & 0 & 0 & -13.00 \\
SIRD5 & Sext & 0.2 & 0 & 0 & -10.209 \\
SIRD6 & Sext & 0.2 & 0 & 0 & -13.00 \\
\hline \hline
\end{tabular}

\section{Local correction}

In order to correct chromaticity locally around the IR, the linear lattice must be able to accommodate the correcting sextupoles in addition to its goal of achieving the design Twiss parameters at the IP $\left(\beta_{x}^{*}\left(\beta_{y}^{*}\right)=0.1(0.02) \mathrm{m}, \alpha_{x, y}^{*}=0, \eta_{x, y}^{*}=\eta_{x, y}^{*}=0\right)$.

The basic guidelines for constructing the interaction region which meets the mentioned goals are stated as the following:

$1 \beta_{\max }$ was minimized to avoid unnecessary chromaticity.

2 Magnet free beam extension $\operatorname{drift}(L=6.5 \mathrm{~m})$ was added after the final focus quadrupoles with negative $\beta$ slope, so the $\beta$ functions was relaxed to a limited amplitude that could be matched easily to the rest of the ring without adding unnecessary chromaticity. 
3 A local chromaticity correction block was introduced. The block features a peak in the betatron function at every $180^{\circ}$ degrees of phase advance. Each peak has a reasonable betatron aspect ratio to ensure uncoupling of the chromatic Montague functions.

4 Dispersion was adjusted to maintain adequate values at the sextupole locations.

5 Sextupoles were added in families; four families for horizontal correction (SIRF1, SIRF2, SIRF3, SIRF4) and six families for vertical chromatic correction (SIRD1, SIRD2, SIRD3, SIRD4, SIRD5, SIRD6). The sextupoles have a symmetry line across the IP. Table (14) presents the sextupoles families and their values. Fig. (42) shows the Montague chromatic functions at the IP and at the beginning of the arc.

6 Sets of octupole magnets were placed adjacent to local correcting sextupoles. They were used for correcting second order tune chromaticity.

TABLE 14: Local correcting sextupole families.

\begin{tabular}{lccc}
\hline \hline Sextupole Family & Number of magnets & $L[\mathrm{~m}]$ & $M\left[\mathrm{~m}^{-3}\right]$ \\
\hline SIRF1 & 2 & 0.2 & 14.2 \\
SIRF2 & 2 & 0.2 & -960 \\
SIRF3 & 4 & 0.2 & 356 \\
SIRF4 & 2 & 0.2 & 356 \\
SIRD1 & 2 & 0.2 & -244 \\
SIRD2 & 2 & 0.2 & 147 \\
SIRD3 & 8 & 0.2 & 356 \\
SIRD4 & 4 & 0.2 & 356 \\
SIRD5 & 4 & 0.2 & 356 \\
SIRD6 & 2 & 0.2 & 356 \\
\hline \hline
\end{tabular}

\section{Global correction}

To correct for tune chromaticity. Sextupoles placed in the arcs were invoked in a global tune correction. Several iterations involving the local correcting sextupoles and octupoles were needed to achieve the required correction. Fig. (43) and (44) show periodic chromatic Montague functions in the entire ring, while Fig. (45) shows 


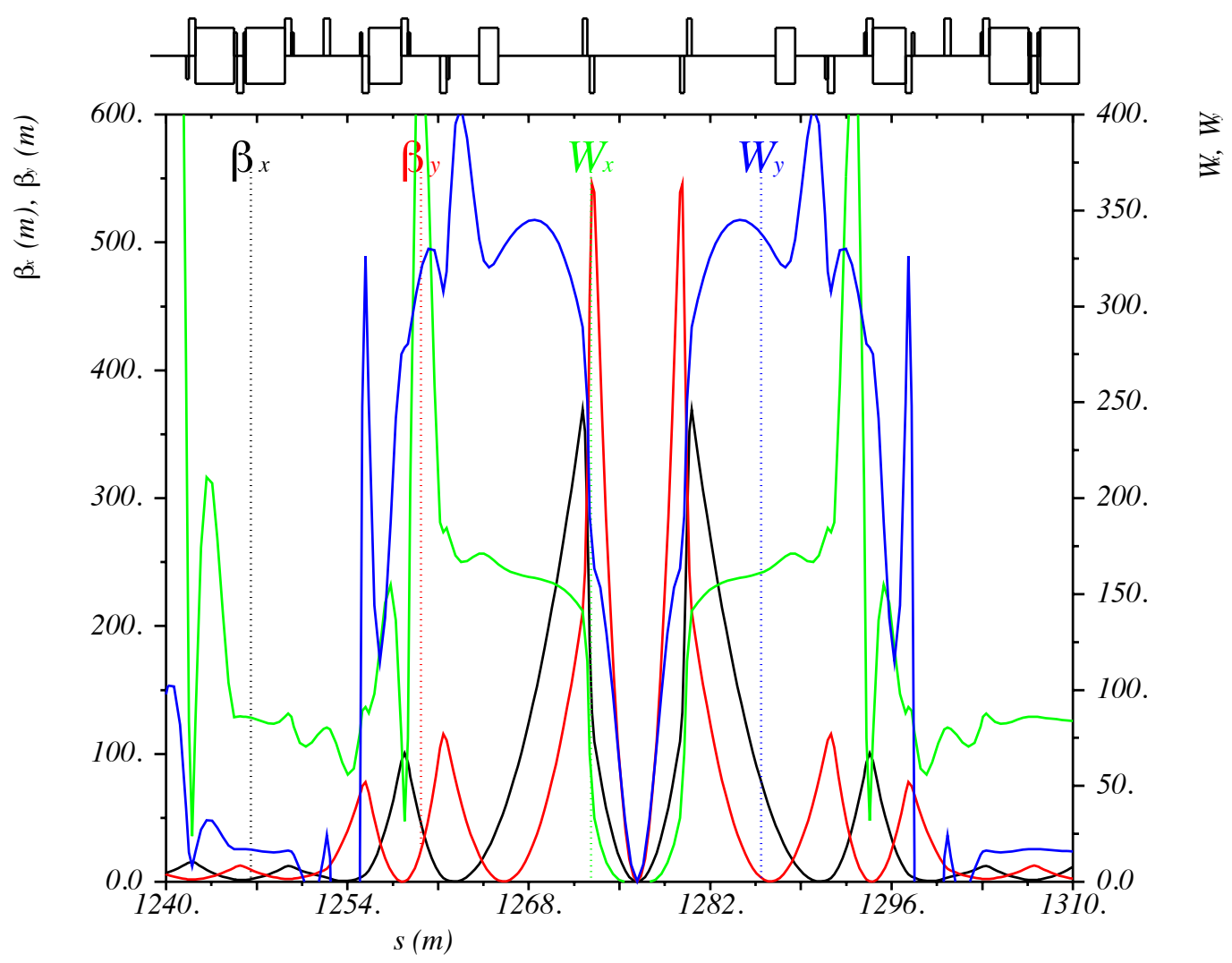

FIG. 42: MEIC Montague chromatic functions at the IP after correction with the local sextupoles.

tune chromaticity after correction. A dynamic aperture tracking study showed that this scheme has more promising dynamic aperture. Fig. (46(a)) shows the dynamic aperture after sextupole correction and Fig. (46(b)) shows the dynamic aperture after adding octupoles for the second order correction. 


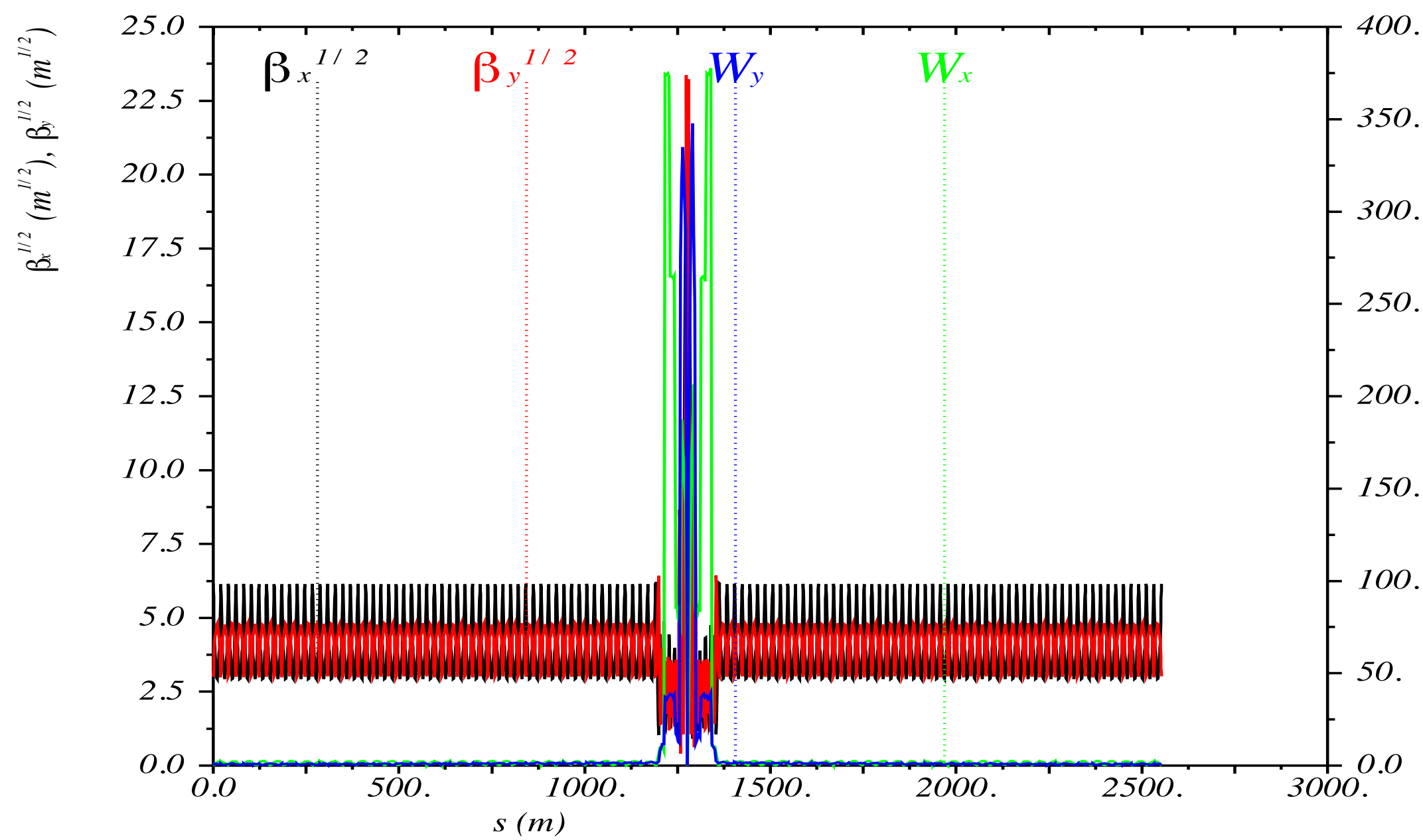

FIG. 43: MEIC Montague chromatic functions after correction with local and global sextupoles. Sextupoles were placed at locations with good aspect ratios between $\beta_{x}$ and $\beta_{y}$ and reasonable dispersion. 




FIG. 44: MEIC Montague chromatic functions in one arc cell after correction.

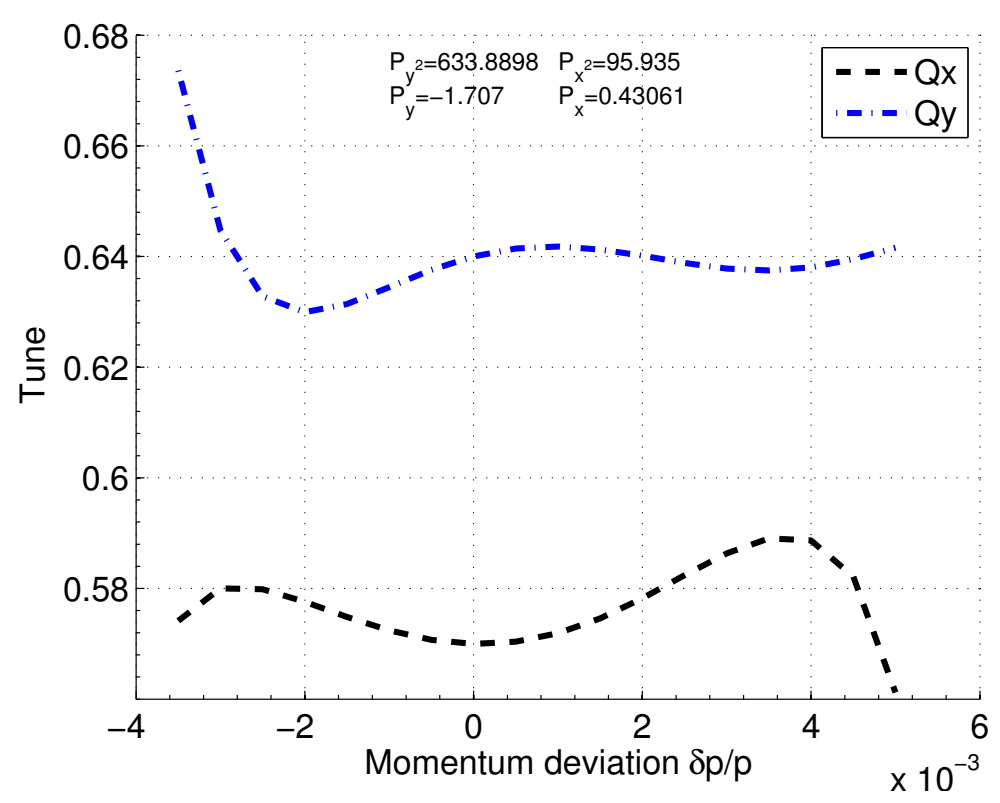

FIG. 45: MEIC tune variation with momentum deviation after correction. Momentum acceptance is $\pm 0.3 \%$ 


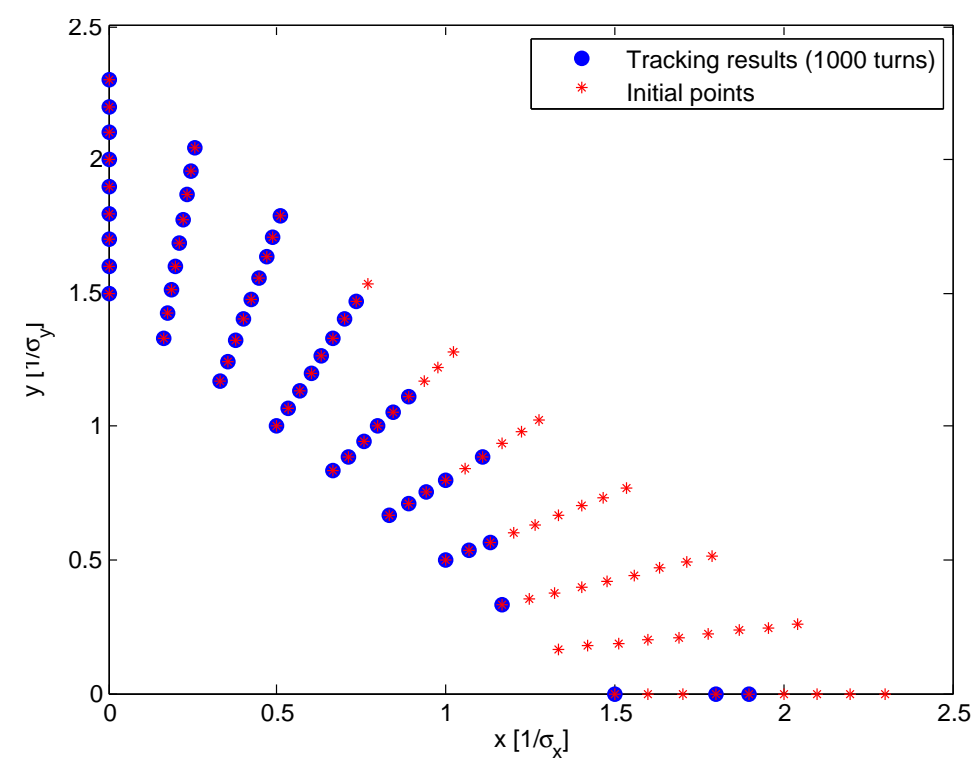

(a) Sextupoles only.

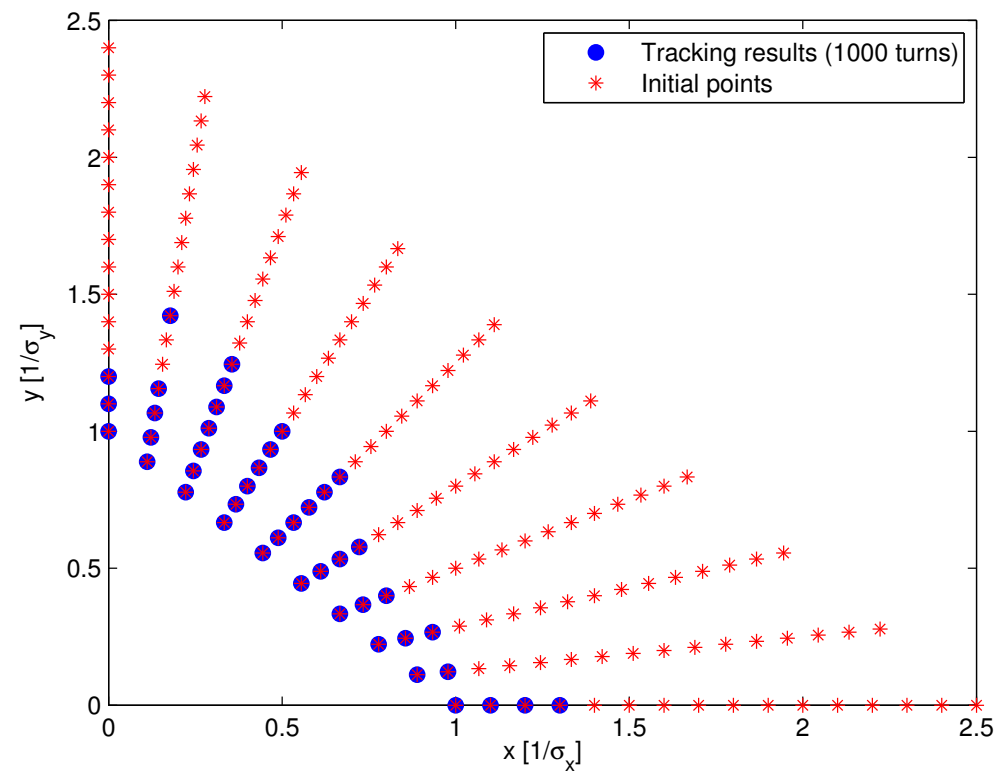

(b) Sextupoles and octupoles.

FIG. 46: Dynamic aperture study for MEIC. Red stars represent initial amplitudes while blue dots represent surviving particles. 


\section{III.5 RESULTS AND DISCUSSION}

This chapter presented the schemes adopted for chromaticity correction for the electron figure- 8 ring of the MEIC beginning by correcting the chromaticity of the rings without the interaction region. This study compared two arc lattice designs, one with a $135^{\circ}$ phase advance per arc cell and the one with a $90^{\circ}$ phase advance per arc cell. Both designs produce a horizontal equilibrium emittance in the range of $4-6 \times 10^{-8}$ m. The $90^{\circ}$ phase advance lattice showed better corrected tune chromaticity with a momentum acceptance of $\pm 2.0 \%$, and dynamic aperture of $120 \sigma_{x, y}$. The $135^{\circ}$ phase advance lattice proved less promising; it produced momentum acceptance of $\pm 0.8 \%$, and dynamic aperture of $85 \sigma_{x, y}$. We conclude that the $90^{\circ}$ phase advance lattice has more potential for electron collider rings in this application. The superior results of the $90^{\circ}$ phase advance lattice could be interpreted by the fact that cell quadrupoles are weaker and thus generate less chromaticity, and they require less sextupole strength to correct them.

Two different designs of the interaction region were explored. In the first case, a non-vanishing dispersion derivative through the interaction region using local and global chromatic correction schemes was studied. The tune chromaticity revealed a momentum acceptance sufficient for 3 hours of Touschek lifetime, but the dynamic aperture was limited. The second one explored a design with vanishing dispersion derivative at the IP, and showed higher correction of tune chromaticity, and a more promising dynamic aperture of $1.5 \sigma_{x, y}$. The symmetric IR showed superior results which can be explained by the fact that the sextupole families were symmetric across the IP since dispersion is symmetric across the IR, and the phase advance across the IR between symmetric sextupoles was $\pi$ which reduced second order effects from local correcting sextupoles.

From the discussion above we conclude that of the cases studied, the $90^{\circ}$ phase advance per arc cell ring including a symmetric IR is the most suitable design for the MEIC electron ring. 


\section{CHAPTER IV}

\section{POLARIZED ELECTRON BEAMS IN FIGURE-8 RINGS}

A unique design feature of a polarized Medium Energy Electron-Ion Collider (MEIC) is its figure-8 storage ring design for both electrons and ions, which significantly simplifies beam polarization maintenance and manipulation. While the electron (positron) polarization is maintained vertical in the arcs of the ring, a stable longitudinal spin at four collision points is achieved through solenoid based spin rotators and horizontal orbit bends. The proposed MEIC lattice was developed in order to preserve a very high polarization (more than $80 \%$ ) of the electron beam injected from the CEBAF machine. The coupled beam trajectory due to solenoids used in the spin rotators was decoupled by a special decoupling insert that works at all solenoid settings.

\section{IV.1 REQUIREMENTS FOR POLARIZED BEAMS FOR MEIC}

There is a growing consensus in the nuclear physics community that further investigations of the quark and gluon structure of matter will require a very high polarization $(70-80 \%)$ for both electron and ion beams.

In the case of the lepton beam, the choice is to inject with full energy from the CEBAF machine and maintain the injected polarization. The polarization of electron beam has to be longitudinal along the momentum vector direction at each of the interaction points. In the arcs in order to take advantage of the Sokolov-Ternov effect the polarization vector has to be vertical.

In the ion case, the polarization has to be longitudinal at the interaction region as well, but since ions do not radiate much synchrotron radiation, the Sokolov-Ternov polarization effect is weak.

\section{IV.2 INTRODUCTION TO POLARIZED BEAMS}

This section will give a brief account on polarized beams in circular accelerators. The Thomas-BMT equation, which describes the particles spin dynamics in particle accelerators, will be presented. 
The first polarized proton acceleration in high-energy $(12 \mathrm{GeV})$ circular accelerators took place at the Zero Gradient Synchrotron (ZGS) at Argonne in the 1970s [32]. This success was followed by a series of high energy particle accelerators with polarized beams. In the 1980s, the AGS at Brookhaven National Laboratory (BNL) started their $24 \mathrm{GeV}$ polarized heavy ion program [33]. The concept of a siberian snake was introduced in the 1970s by S. Derebenv and Kondratenko [34], opening the possibility of achieving higher energies. The principle of siberian snakes with a low-energy polarized proton beam was tested at the Indiana University Cyclotron Facility (IUCF) in 1989 [35]. The ability to use partial siberian snakes and radiofrequency dipoles in order to maintain polarization during acceleration in the AGS was demonstrated in 1999 [36]. Later, the acceleration of very polarized protons to higher energies $(100 \mathrm{GeV}-205 \mathrm{GeV})$ with $30 \%$ polarization was accomplished [37] [38].

\section{IV.2.1 Thomas-BMT equation}

Thomas-BMT equation describes the motion for a spin vector defined in the rest frame of the particle in a synchrotron and it is given by [39]

$$
\frac{d \vec{S}}{d t}=\frac{e}{\gamma m} \times\left[(1+G \gamma) \vec{B}_{\perp}+(1+G) \vec{B}_{\|}+\left(G \gamma+\frac{\gamma}{\gamma+1}\right) \frac{\vec{E} \times \vec{\beta}}{c}\right],
$$

where $\vec{S}$ is the spin vector of the particle in its rest frame, $\vec{B}_{\|}$and $\vec{B}_{\perp}$ are the longitudinal and transverse components of the magnetic fields in the laboratory frame with respect to particle momentum direction, $\vec{E}$ is the electric field, and $\vec{\beta}$ and $\gamma$ are relativistic factors. $G$ is the anomalous gyromagnetic $g$-factor which is given by

$$
G=\frac{g}{2}-1
$$

The spin equation of motion in the rest frame of the particle in presence of an external magnetic field $(\vec{B})$ is given by

$$
\frac{d \vec{S}_{R}}{d t}=g \frac{e}{2 m} \vec{S}_{R} \times \vec{B}
$$

where $\overrightarrow{S_{R}}$ is the particles spin vector in the rest $(\mathrm{R})$ frame.

In case of spin particle moving in a circular orbit with transverse magnetic fields in the laboratory frame, one can derive the spin 4-vector in the Lorentz boosted frame given by [39], 


$$
\left(S_{0 L}, \overrightarrow{S_{L}}\right)=\left(\gamma \vec{\beta} \cdot \vec{S}_{R}, \vec{S}_{R}+\frac{\gamma^{2}}{\gamma+1} \vec{\beta}\left(\vec{\beta} \cdot \vec{S}_{R}\right)\right)
$$

which shows the relation between the spin vector in laboratory frame and spin vector in the particle's rest frame. The Thomas-BMT equation is derived easily from Eq. (107) [39].

From the Thomas-BMT equation we can see that in a solenoid magnet, $\vec{B}_{\|}$produces a spin rotation around the longitudinal direction, and it can rotate horizontal polarization into vertical polarization or vice versa. The spin rotation in a longitudinal field is energy dependent therefore spin rotations should be done at low energies if possible. In a dipole field, $\vec{B}_{\perp}$, the equation of spin motion relative to the particle motion shows that spin rotation relative to the orbit motion is independent of energy.

\section{IV.2.2 Radiative Polarization (Sokolov-Ternov Effect)}

Circulation of electron and positron beams for a relatively long time in a storage ring causes a polarization build up due to the reaction of continuous emission of transversely polarized synchrotron radiation [11].

The polarization time is given by [11]

$$
\frac{1}{\tau_{p}}=\frac{5 \sqrt{3}}{8} \frac{e^{2} \hbar \gamma^{5}}{m^{2} c^{2} \rho^{3}},
$$

where $e$ and $m$ are the electron charge and mass respectively, and $\rho$ is the radius of curvature of the storage ring. The theoretically maximum achievable polarization is $92.38 \%$. The polarization time is a strong function of beam energy and is very

long for low energies. Several GeVs of energy is required for the polarization time to become short compared to the storage time of an electron beam in a storage ring.

\section{IV.3 COUPLED MOTION IN A SOLENOID FIELD}

The spin rotators in MEIC include solenoid magnets. The longitudinal magnetic field inside the solenoid magnets rotate the beam by an angle which depends in the solenoid field strength. In this section, we introduce the linear equation of motion inside a solenoid field.

The linear equation of motion in a solenoid magnetic field can be derived from 
the Hamiltonian of Eq. (27), and is given by

$$
\begin{aligned}
& x^{\prime \prime}=S(s) y^{\prime}+\frac{1}{2} S^{\prime}(s) y \\
& y^{\prime \prime}=-S(s) B_{s} x^{\prime}-\frac{1}{2} S^{\prime}(s) x,
\end{aligned}
$$

where $S(s)=e B_{s} / p$. Following [40], we can derive the transformation matrix of a solenoid magnet as follows. Perform a coordinate rotation in complex plane. Define $R$ to be

$$
R=(x+i y) e^{-i \phi(s)} .
$$

Eqs. (109) can be combined in to one equation

$$
(x+i y)^{\prime \prime}+i S(s)(x+i y)^{\prime}+i \frac{1}{2} S^{\prime}(s)(x+i y)=0 .
$$

Applying Eq. (110) to Eq. (111)

$$
\begin{gathered}
(x+i y)^{\prime}=R^{\prime} e^{i \phi}+i \phi^{\prime} R e^{i \phi} . \\
(x+i y)^{\prime \prime}=R^{\prime \prime} e^{i \phi}+i 2 \phi^{\prime} R^{\prime} e^{i \phi}+i \phi^{\prime \prime} R e^{i \phi}-\phi^{\prime 2} R e^{i \phi} .
\end{gathered}
$$

Using Eq. (110)

$$
R^{\prime \prime}-\left[S(s) \phi^{\prime}+\phi^{\prime 2}\right] R+i 2\left[\phi^{\prime}+\frac{1}{2} S(s)\right] R^{\prime}+i\left[\phi^{\prime \prime}+\frac{1}{2} S^{\prime}(s)\right] R=0
$$

is obtained.

Assuming continuous rotation along the solenoid axis with an angle defined by

$$
\phi(s)=-\frac{1}{2} \int_{s_{0}}^{s} S(\zeta) d \zeta
$$

the equation of motion in terms of $R$ becomes

$$
R^{\prime \prime}+\frac{1}{4} S^{2}(s) R=0
$$

where $R$ can be represented as $R=v+i w$. At the entrance of the solenoid magnet, $\phi=0, v_{0}=x_{0}$, and $w_{0}=y_{0}$. Applying Eq. (116) to the equation of motion, two uncoupled equations of motion are obtained:

$$
\begin{aligned}
& v^{\prime \prime}+\frac{1}{4} S^{2}(s) v=0, \\
& w^{\prime \prime}+\frac{1}{4} S^{2}(s) w=0 .
\end{aligned}
$$


The motion of the particle in a solenoid can be represented by a quadrupole of strength, $k_{s}$, followed by a coordinate system rotation by angle, $-\phi\left(L_{s}\right)$, where $k_{s}=$ $\frac{1}{4} S^{2}\left(L_{s}\right)$.

The solution of the equation of motion may be expressed in a matrix formulation given by the following form:

$$
\left(\begin{array}{c}
x(s) \\
x^{\prime}(s) \\
y(s) \\
y^{\prime}(s)
\end{array}\right)=\left(\begin{array}{llll}
M_{11} & M_{12} & M_{13} & M_{14} \\
M_{21} & M_{22} & M_{23} & M_{24} \\
M_{31} & M_{32} & M_{33} & M_{34} \\
M_{41} & M_{42} & M_{43} & M_{44}
\end{array}\right)\left(\begin{array}{c}
x_{0} \\
x_{0}^{\prime} \\
y_{0} \\
y_{0}^{\prime}
\end{array}\right)
$$

where the transformation matrix elements $M_{i j}$ are functions of the field strength of the magnet.

The transformation matrix of the solenoid focusing effect from the beginning of solenoid to point $s$ inside the solenoid is

$$
M_{s}^{f}\left(s_{0} \mid s\right)=\left(\begin{array}{cccc}
\cos \phi & \frac{2}{S} \sin \phi & 0 & 0 \\
\frac{-S}{2} \sin \phi & \cos \phi & 0 & 0 \\
0 & 0 & \cos \phi & \frac{2}{S} \sin \phi \\
0 & 0 & \frac{-S}{2} \sin \phi & \cos \phi
\end{array}\right)
$$

where $\phi=\frac{1}{2} S s$. The rotation matrix of the solenoid including the rotation effect is given by

$$
M_{s}^{r}\left(s_{0} \mid s\right)=\left(\begin{array}{cccc}
\cos \phi & 0 & \sin \phi & 0 \\
\frac{-S}{2} \sin \phi & \cos \phi & \frac{S}{2} \cos \phi & \sin \phi \\
-\sin \phi & 0 & \cos \phi & 0 \\
\frac{S}{2} \cos \phi & -\sin \phi & \frac{-S}{2} \sin \phi & \cos \phi
\end{array}\right)
$$

The total transformation matrix from beginning of the solenoid magnet to the point $s$ is given by multiplication of the last two matrices;

$$
M_{s}\left(s_{0} \mid s\right)=\left(\begin{array}{cccc}
\cos ^{2} \phi & \frac{1}{S} \sin 2 \phi & \frac{1}{2} \sin 2 \phi & \frac{2}{S} \sin ^{2} \phi \\
\frac{-S}{2} \sin 2 \phi & \cos 2 \phi & \frac{S}{2} \cos 2 \phi & \sin 2 \phi \\
\frac{-1}{2} \sin 2 \phi & \frac{-2}{S} \sin ^{2} \phi & \cos ^{2} \phi & \frac{1}{S} \sin 2 \phi \\
\frac{-S}{2} \cos 2 \phi & -\sin 2 \phi & \frac{-S}{2} \sin 2 \phi & \cos 2 \phi
\end{array}\right) .
$$

To add the effect of the solenoid fringe field assuming hard edge approximation, the 
transformation matrix at the end of the solenoid is given by

$$
M_{s}(0 \mid L)=\left(\begin{array}{cccc}
\cos ^{2} \Phi & \frac{1}{S} \sin 2 \Phi & \frac{1}{2} \sin 2 \Phi & \frac{2}{S} \sin ^{2} \Phi \\
-\frac{S}{4} \sin 2 \Phi & \cos ^{2} \Phi & -\frac{S}{2} \sin ^{2} \Phi & \frac{1}{2} \sin 2 \Phi \\
-\frac{1}{2} \sin 2 \Phi & -\frac{2}{S} \sin ^{2} \Phi & \cos ^{2} \Phi & \frac{1}{S} \sin 2 \Phi \\
\frac{S}{2} \sin ^{2} \Phi & -\frac{1}{2} \sin 2 \Phi & -\frac{S}{4} \sin 2 \Phi & \cos ^{2} \Phi
\end{array}\right)
$$

where $\Phi=S L / 2$. To focus a parallel beam at the entrance of the solenoid to focal point at the end of the solenoid, the total rotation angle has to be $\pi / 2$, i.e. $\Phi=S L / 2=\pi / 2$.

\section{IV.4 GENERALIZING THE COURANT-SNYDER PARAMETRIZA- TION TO TWO DIMENSIONAL COUPLED LINEAR MOTION}

The Courant-Snyder parameterization of one-dimensional linear betatron motion has been generalized to two-dimensional coupled linear motion [41]. Four betatron functions, four alpha-functions, and two betatron phase advances are needed to represent the $4 \times 4$ symplectic transfer matrix. Those ten parameters provide a generalization to Courant-Snyder formalism analogous to the uncoupled case.

A single-particle phase-space trajectory along the beam orbit is

$$
\hat{X}(s)=\operatorname{Re}\left(\sqrt{\varepsilon_{1}} \hat{V}_{1}(s) e^{-i\left(\psi_{1}+\mu_{1}(s)\right)}+\sqrt{\varepsilon_{2}} \hat{V}_{2}(s) e^{-i\left(\psi_{2}+\mu_{2}(s)\right)}\right),
$$

where $\hat{M}(0, L)$ is the transfer matrix of the entire ring, and $\hat{V}_{1,2}$ are the eigen-vectors of the transfer matrix, $\hat{M}(0, s) \hat{M} \hat{M}^{-1}(0, s)$, given by

$$
\hat{V}_{1,2}(s)=e^{i \mu_{1,2}(s)} \hat{M}(0, s) \hat{V}_{1,2} .
$$

$\psi_{1,2}$ are the initial phases of the betatron motion. The eigen vectors can be introduced in the following standard form:

$$
\begin{gathered}
\hat{V}_{1}(s)=\left(\begin{array}{c}
\sqrt{\beta_{1 x}(s)} \\
-\frac{1}{\sqrt{\beta_{1 x}}}\left(i(1-u)+\alpha_{1 x}\right) \\
\sqrt{\beta_{1 y}} e^{i v_{1}} \\
-\frac{1}{\sqrt{\beta_{1 y}}}\left(i u+\alpha_{1 y}\right) e^{i v_{1}}
\end{array}\right) \\
\hat{V}_{2}(s)=\left(\begin{array}{c}
\sqrt{\beta_{2 x}(s)} e^{i v_{2}} \\
-\frac{1}{\sqrt{\beta_{2 x}}}\left(i u+\alpha_{2 x}\right) e^{i v_{2}} \\
\sqrt{\beta_{2 y}} \\
-\frac{1}{\sqrt{\beta_{2 y}}}\left(i(1-u)+\alpha_{2 y}\right)
\end{array}\right),
\end{gathered}
$$


where $u$ is defined in terms of $k_{x}$ and $k_{y}$ :

$$
\begin{aligned}
& k_{x}=\sqrt{\frac{\beta_{2 x}}{\beta_{1 x}}}, \\
& k_{y}=\sqrt{\frac{\beta_{1 y}}{\beta_{2 y}}},
\end{aligned}
$$

and

$$
\begin{aligned}
& A_{x}=k_{x} \alpha_{1 x}-k_{x}^{-1} \alpha_{2 x}, \\
& A_{y}=k_{y} \alpha_{2 y}-k_{y}^{-1} \alpha_{1 y}
\end{aligned}
$$

and

$$
u=\frac{1}{1-\left(k_{x} k_{y}\right)^{2}}\left[-k_{X}^{2} k_{y}^{2} \pm \sqrt{k_{X}^{2} k_{y}^{2}\left(1+\frac{A_{X}^{2}-A_{y}^{2}}{k_{x}^{2}-k_{y}^{2}}\left(1-k_{X}^{2} k_{y}^{2}\right)\right)} .\right.
$$

$v_{1,2}$ are given by

$$
\begin{aligned}
& e^{i\left(v_{1}+v_{2}\right)}=\frac{A_{x}+i\left(k_{x}(1-u)+k_{x}^{-1} u\right)}{A_{y}-i\left(k_{y}(1-u)+k_{y}^{-1} u\right)}, \\
& e^{i\left(v_{1}+v_{2}\right)}=\frac{A_{x}+i\left(k_{x}(1-u)+k_{x}^{-1} u\right)}{A_{y}+i\left(k_{y}(1-u)-k_{y}^{-1} u\right)} .
\end{aligned}
$$

In the case of weak coupling, one should normally choose $\hat{V}_{1}$ as the eigen-vector which mainly relates to the horizontal motion, and $\hat{V}_{2}$ to the vertical motion. In the case of strong coupling the choice is arbitrary. Such a parameterization works equally well for weak and strong coupling and can be useful for analysis of coupled betatron motion in circular accelerators, as well as in transfer lines.

Here $\beta_{1 x}, \beta_{1 y}, \beta_{2 x}$, and $\beta_{2 y}$ are the beta-functions: The alphas $\alpha_{1 x}, \alpha_{1 y}, \alpha_{2 x}$, and $\alpha_{2 y}$, are negative half-derivatives of the betas at regions with zero longitudinal magnetic field and there are two phases $v_{1}$, and $v_{2}$. These ten independent parameters are called the generalized Twiss functions.

\section{IV.5 POLARIZATION AROUND THE FIGURE-8 RING}

In MEIC longitudinally polarized electrons (positrons) are generated by a polarized photoinjector and then accelerated to the desired energy in CEBAF. After that, they are injected into the electron storage ring with vertical polarization in the arcs and accumulated there until their average current reaches a desired value, taking advantage of the Sokolov-Ternov effect [11]. While electron polarization is maintained 
vertical in the arcs of the ring, a stable longitudinal spin at all four collision points is required by experiments and is achieved through solenoid based spin rotators and horizontal beam orbit bends integrated into some of the spin rotators. The ions are injected in one of the IR of the ion ring with longitudinal polarization. Three identical Siberian Snakes make the longitudinal polarization periodic in both interaction regions and provide a very efficient spin tune control.

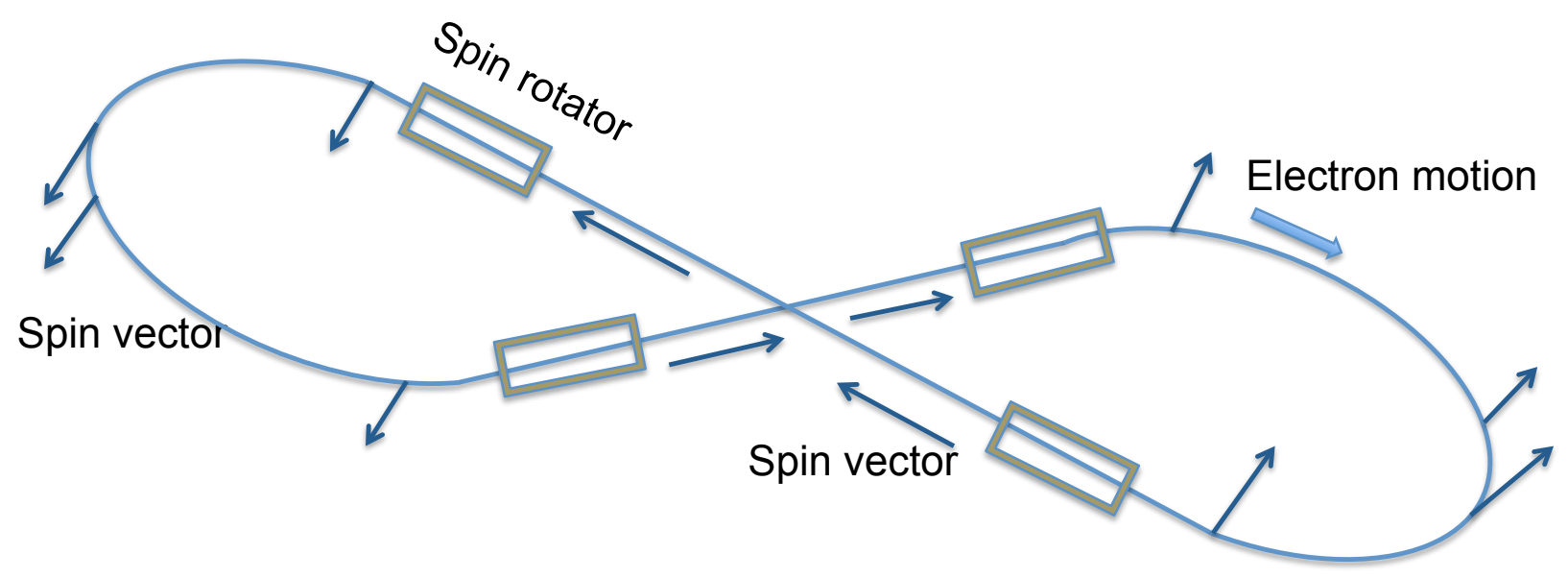

FIG. 47: Spin vector for the electron beam polarization around the figure- 8 ring

\section{IV.6 UNIVERSAL SPIN ROTATORS}

A generic spin rotation scheme has been designed to transform the electron spin in MEIC from vertical in the arcs to longitudinal at all interaction points. The scheme, which is called the Universal Spin Rotator (USR), does not change the beam orbit for all planned electron beam energies and consists of a combination of superconducting solenoids and horizontal arc dipoles. One possible implementation of the USR is shown in Fig. (48). The last two equal arc dipole sections, B1 and B2, interleave with two solenoids (Sol I and Sol II). The rotator works by adjusting spin rotation angles in solenoids depending on the beam energy. To provide the required spin rotation in the whole $(3-9 \mathrm{GeV})$ energy range, the bending angle of each dipole section is $6.6^{\circ}$ and the total integral field of each solenoid is 48 Tesla meter [42]. X-Y betatron coupling introduced by solenoids is compensated by the methods based on $[43,44]$. 


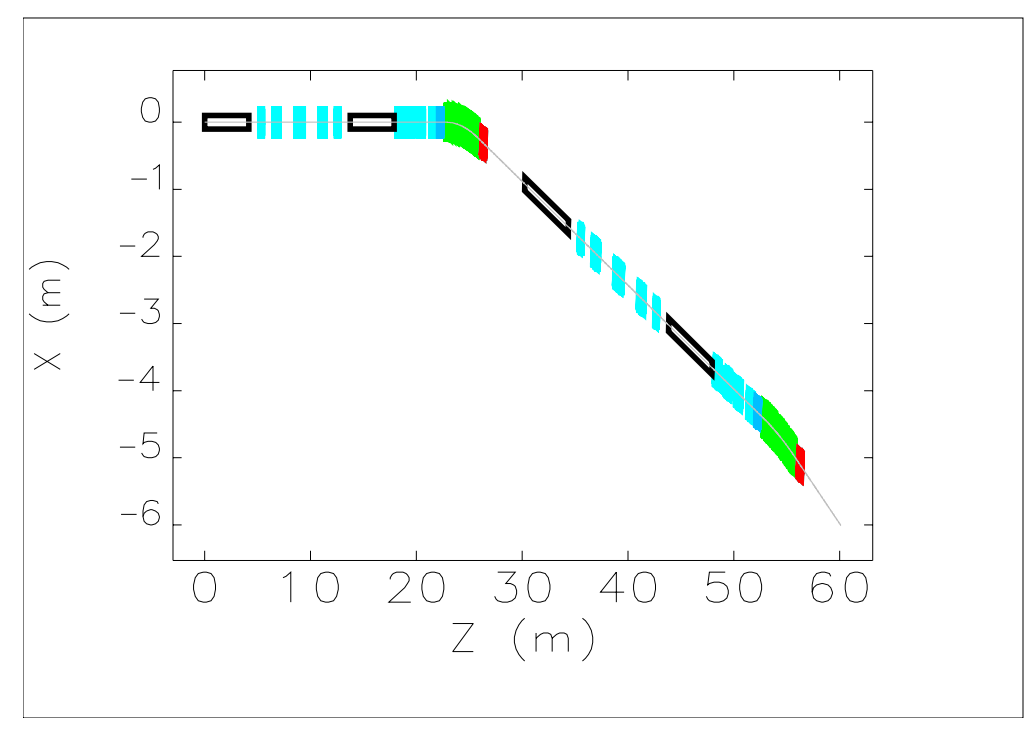

FIG. 48: Layout of the Universal Spin Rotator (USR) (solenoids are black, quadrupoles are blue and dipoles are green)

\section{IV.7 COUPLING COMPENSATION INSERT}

Transverse beam coupling introduced by the solenoids is compensated locally. Each solenoid is divided into two equal parts and a set of quadrupoles is inserted between them to cancel out the coupling. In order to automatically cancel the orbit coupling, the overall transfer matrix of the insert may have a structure of $\left(M_{C O M P}\right)$, as described by the following equation:

$$
M_{C O M P}=\left(\begin{array}{cc}
M & 0 \\
0 & -M
\end{array}\right),
$$

where $M$ is a general $2 \times 2$ matrix. The overall transfer matrix of two identical solenoids with the insert in between will result in an uncoupled cumulative transfer matrix given by the following form

$$
M_{s o l} \cdot M_{C O M P} \cdot M_{s o l}=\left(\begin{array}{cc}
A & 0 \\
0 & B
\end{array}\right),
$$

which is independent of the solenoid field strength ( $A$ and $B$ are $2 \times 2$ matrices).

We examine the special case where an insert which has the following transfer matrix, corresponding to a phase advance of $180^{\circ}$ in both planes 
TABLE 15: Magnet parameters for universal spin rotator (USR) at different beam energies. $\phi_{1,2}$ and DBL are the solenoid spin rotation angle and integral field strength respectively. $\theta_{1,2}$ are the dipole spin rotation angles.

\begin{tabular}{ccccccc}
\hline \hline $\mathbf{E}$ & \multicolumn{2}{c}{ Sol. I } & \multicolumn{2}{c}{ Sol. II } & \multicolumn{2}{c}{ Spin Rot. } \\
\hline $\mathrm{GeV}$ & $\phi_{1}[\mathrm{rad}]$ & DBL [Tesla $\mathrm{m}]$ & $\phi_{2}[\mathrm{rad}]$ & DBL $[\mathrm{T} \mathrm{m}]$ & $\theta_{1}[\mathrm{rad}]$ & $\theta_{2}[\mathrm{rad}]$ \\
\hline 3 & $\pi / 2$ & 15.7 & 0 & 0 & $\pi / 3$ & $\pi / 6$ \\
4.5 & $\pi / 4$ & 11.8 & $\pi / 2$ & 23.6 & $\pi / 2$ & $\pi / 4$ \\
6 & 0.63 & 12.3 & $\pi-1.23$ & 38.2 & $2 \pi / 3$ & $\pi / 3$ \\
9 & $\pi / 6$ & 15.7 & $2 \pi / 3$ & 62.8 & $\pi$ & $\pi / 2$ \\
12 & 0.62 & 24.6 & $\pi-1.23$ & 76.4 & $4 \pi / 3$ & $2 \pi / 3$ \\
\hline \hline
\end{tabular}

$$
M_{C O M P}=\left(\begin{array}{cccc}
1 & 0 & 0 & 0 \\
0 & 1 & 0 & 0 \\
0 & 0 & -1 & 0 \\
0 & 0 & 0 & -1
\end{array}\right)
$$

The total transfer matrix for the two solenoid halves and the coupling compensating insert will be given by

$M_{\text {sol }} \cdot M_{C O M P} \cdot M_{\text {sol }}=$

$$
\left(\begin{array}{cccc}
\cos 2 \Phi & \frac{4}{S} \sin \Phi \cos \Phi & 0 & 0 \\
-S \sin \Phi \cos \Phi & \cos 2 \Phi & 0 & 0 \\
0 & 0 & -\cos 2 \Phi & -\frac{4}{S} \sin \Phi \cos \Phi \\
0 & 0 & S \sin \Phi \cos \Phi & -\cos 2 \Phi
\end{array}\right)
$$

Eq. (133) shows total cancelation of the coupling terms because the matrix is now block diagonal.

Consider the more general matrix of the form defined by the arbitrary constants $a$ and $b$, 


$$
\left(\begin{array}{cccc}
a & 0 & 0 & 0 \\
0 & b & 0 & 0 \\
0 & 0 & -a & 0 \\
0 & 0 & 0 & -b
\end{array}\right)
$$

The total transfer matrix will be $M_{\text {sol }} \cdot M_{C O M P} \cdot M_{\text {sol }}=$

$$
\left(\begin{array}{cccc}
M_{11} & \frac{1}{S}(a+b) \sin 2 \Phi & 0 & 0 \\
-\frac{1}{4} S(a+b) \sin 2 \Phi & M_{22} & 0 & 0 \\
0 & 0 & -M_{11} & -\frac{1}{S}(a+b) \sin 2 \Phi \\
0 & 0 & \frac{1}{4} S(a+b) \sin 2 \Phi & -M_{22}
\end{array}\right)
$$

where $M_{11}=((a+b) \cos 2 \Phi+a-b) / 2$, and $M_{22}=((a+b) \cos 2 \Phi-a+b) / 2$.

The existence of four spin rotators with relatively long solenoids in MEIC makes preserving the modularity of the spin rotators and matching to the rest of the ring challenging tasks. Existing schemes involve at least seven normal quadrupoles, and skew quadrupoles are conceivable [44]. Unfortunately such schemes are not compact enough to fit the MEIC layout, which leads us to the following new design for the compensation system.

The new design involves the minimal required optimization parameters needed to fulfill four conditions given in Eq. (130). One could note that the symplecticity of the system will reduce those conditions to only three, as the symplecticity of the transformation introduces a condition on the matrix elements. A set of two symmetric doublets separated by one singlet quadrupole is enough to meet the three conditions. The compactness of such a system was incorporated in the optimization process yielding relatively short drifts between the quadrupoles as seen in Fig. (48).

The $\beta$-functions through one of the compensated solenoids are shown in Fig. (49). Similarly, $\beta$-functions through the whole spin rotator with the horizontal dipoles are shown in Fig. (50), where one can notice that vertical dispersion was generated by coupling introduced by first half of the solenoid and then compensated back to zero by the end of the second half of the solenoid.

The structure of the coupling compensation insert is given by

CSOL,OS1,QDS,OS2,QFS,OS3,QDS2,OS3,QFS,OS2,QDS,OS1,CSOL 
where the magnet parameters are given in Table (16), and CSOL is one half of the solenoid magnet.

TABLE 16: Magnets of the coupling compensation insert.

\begin{tabular}{cccc}
\hline \hline Element & Type & $L[\mathrm{~m}]$ & $K\left[\mathrm{~m}^{-2}\right]$ \\
\hline QDS & QUADRUPOLE & 0.25 & 2.4 \\
QFS & QUADRUPOLE & 0.51 & -1.25 \\
QDS2 & QUADRUPOLE & 0.70 & 1.24 \\
OS1 & DRIFT & 1.0 & 0 \\
OS2 & DRIFT & 1.0 & 0 \\
OS3 & DRIFT & 1.5 & 0 \\
\hline \hline
\end{tabular}

\section{IV.8 INTEGRATION OF SPIN ROTATORS TO MEIC}

The symmetric insert has the advantage of requiring only four parameters to match the insert to the end of the arc and to the FODO cells of the straight. This will reduce the number of matching quadrupoles to four. Fig. (52(a)) shows the Twiss functions are matched from the arc to the first USR then the matched to the straight section, and for the second USR in the same straight to the second arc section as seen in Fig. (52(b)). 


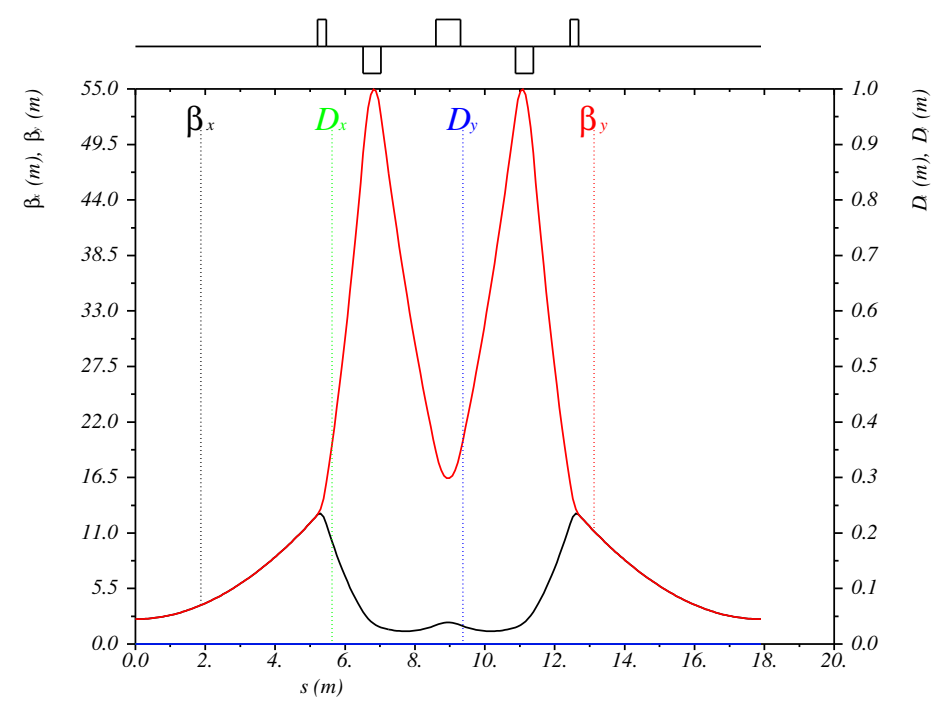

FIG. 49: $\beta$-functions across the symmetric insertion between two identical solenoids.

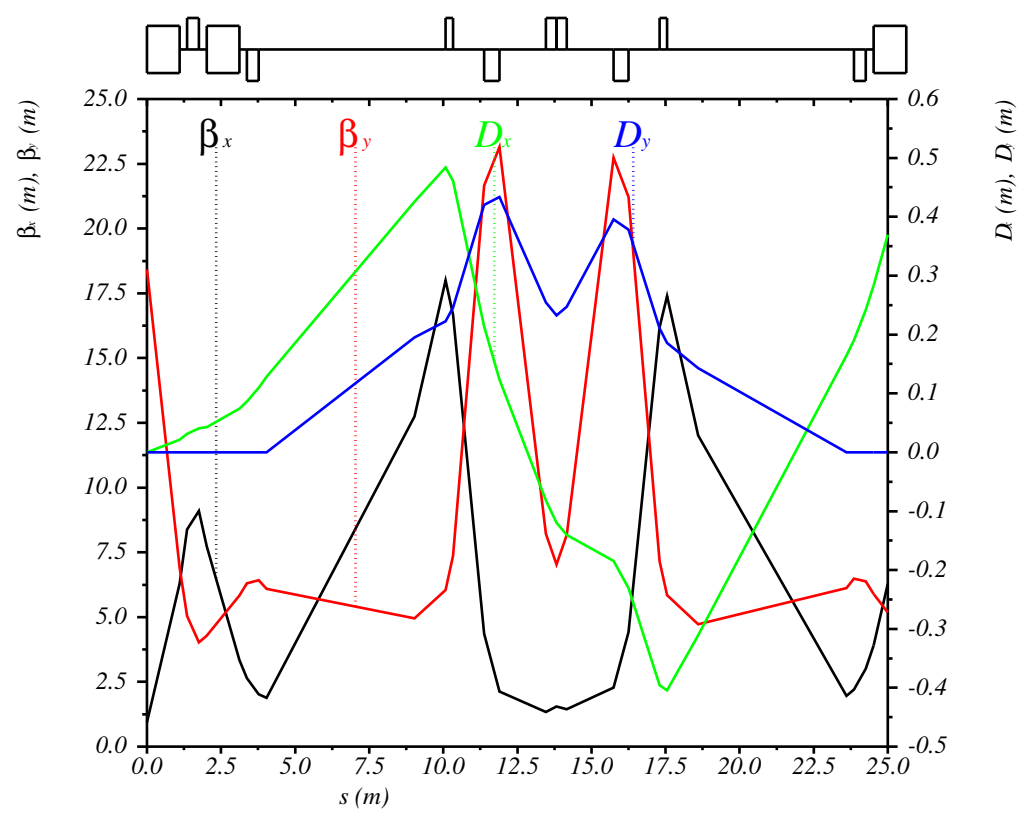

FIG. 50: Dispersion function across the USR. One can see that although the electron ring is a totally horizontal, the horizontal dispersion passing through the solenoid is coupled and vertical dispersion is generated. Vertical dispersion is suppressed by the end of the second half of the solenoid due to rotation introduced by the coupling compensation insert. 


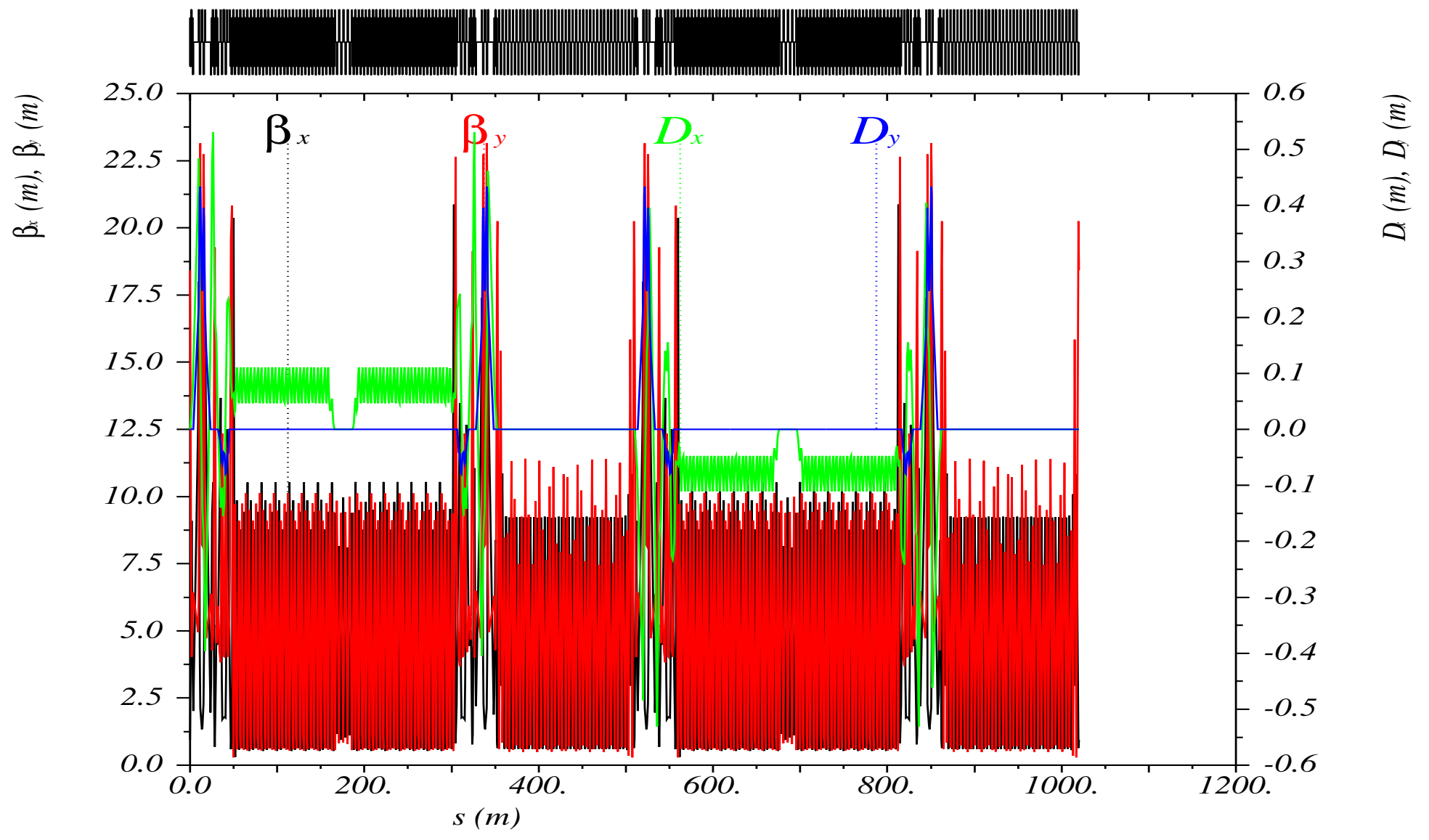

FIG. 51: Twiss functions through the MEIC figure-8 ring with the integrated and matched USR. 


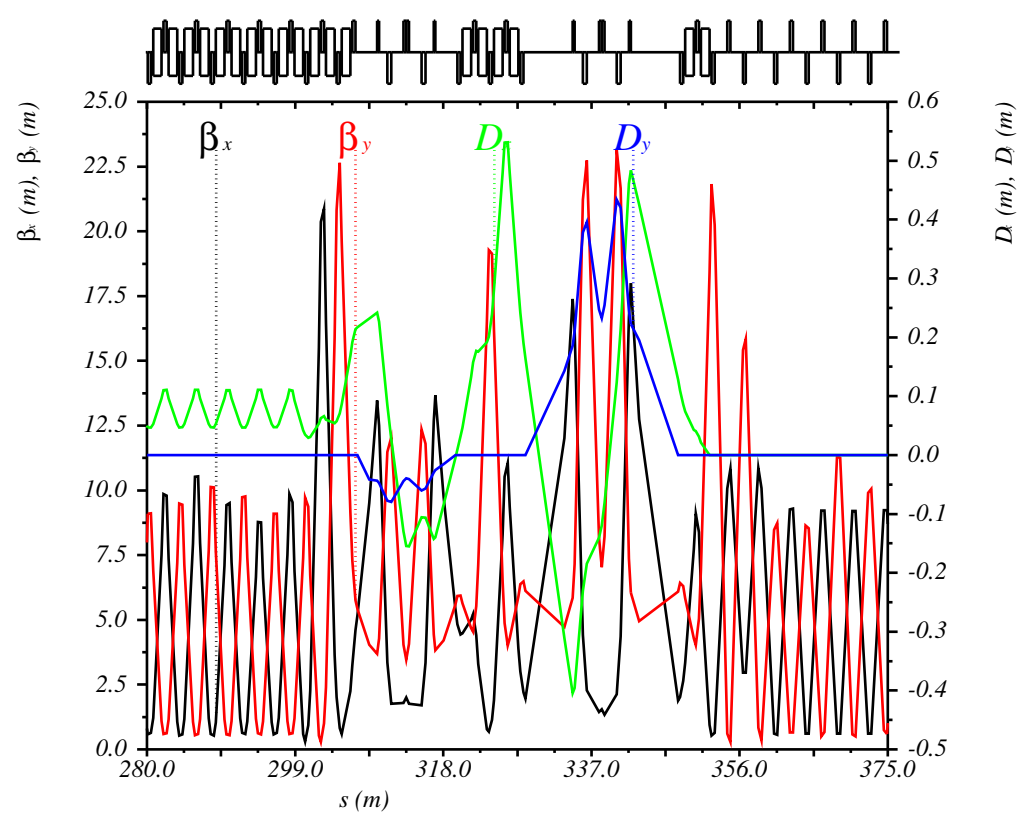

(a) USR was matched at the end of the first arc section to the start of the straight section.

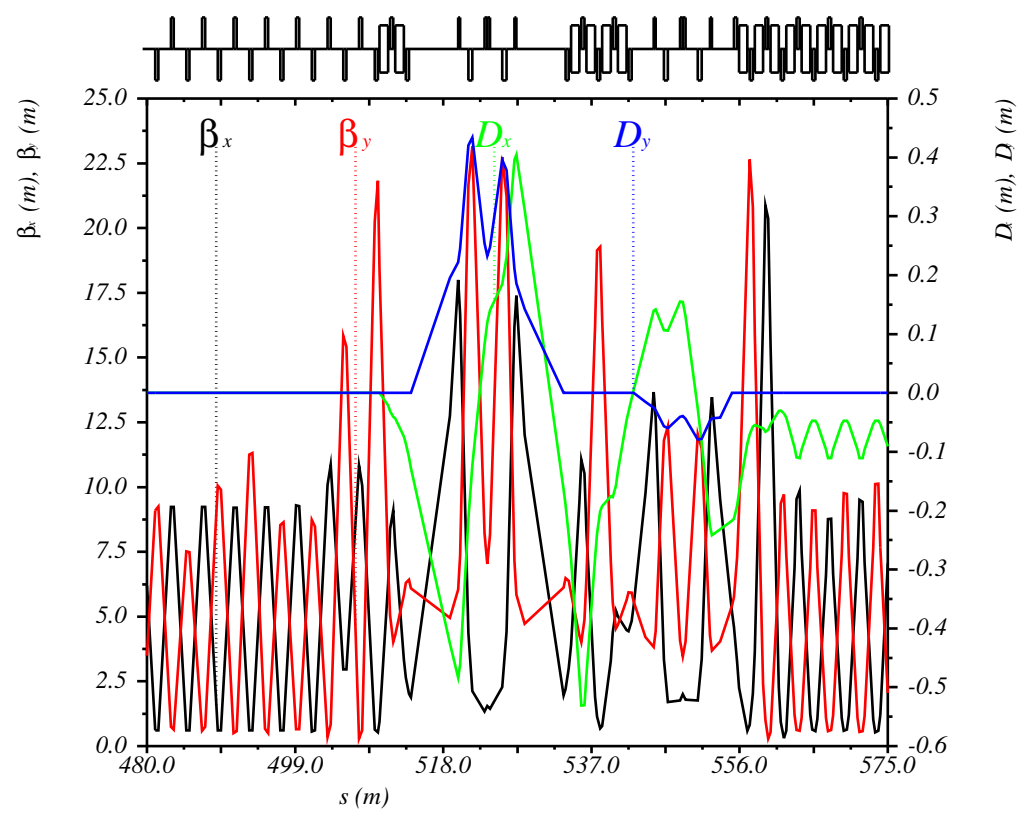

(b) USR was matched at the end of the straight section to the start of the second arc section.

FIG. 52: Twiss functions through the USR. The USR was matched at the end of the arc section and to the start of the straight section. 


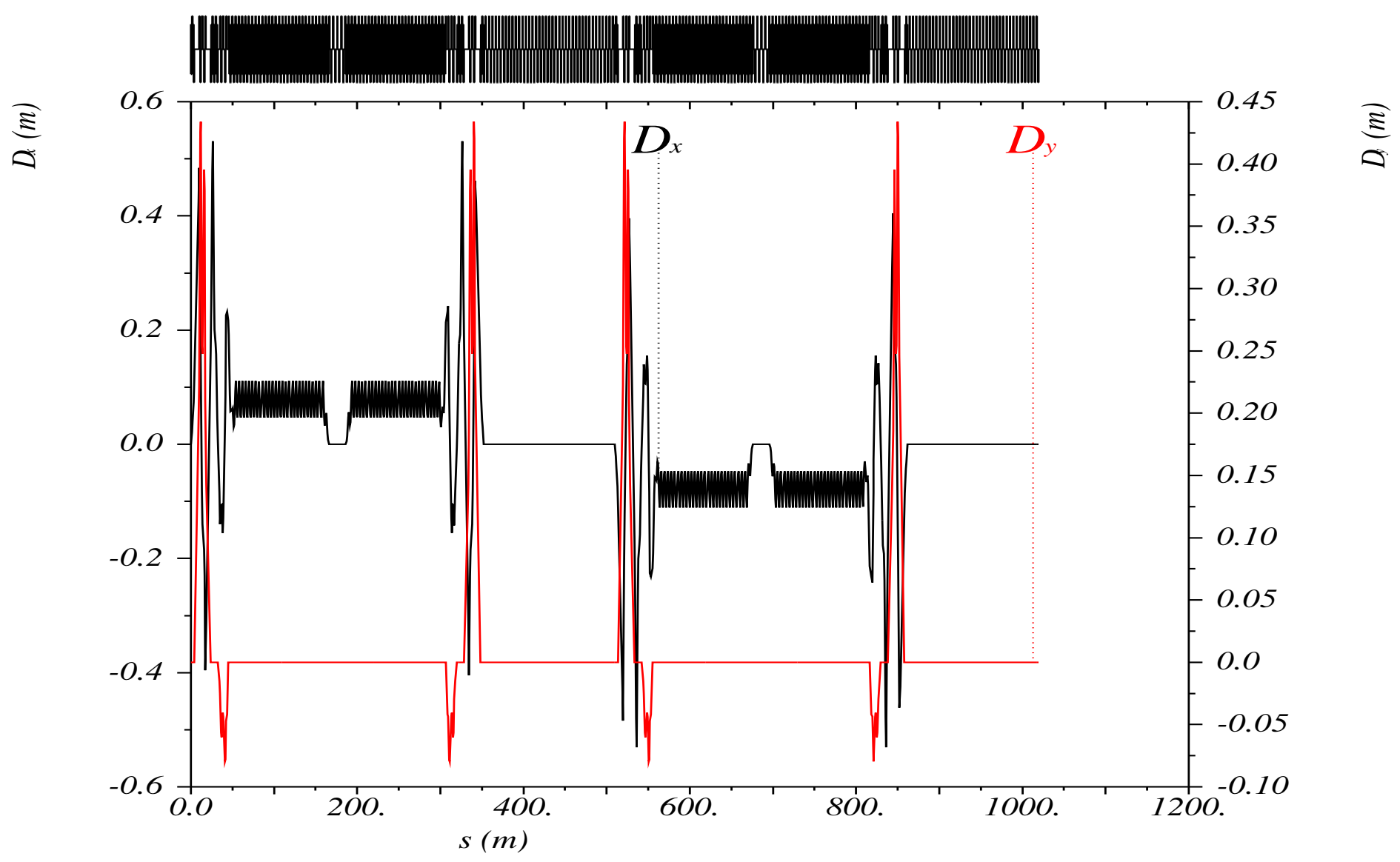

FIG. 53: Dispersion through the MEIC figure-8 ring with the integrated and matched USR. Note that the vertical dispersion vanishes at the end of the USR. 


\section{IV.8.1 Stability study}

A stability study of the coupling compensation insert was carried out. An error of the order of $10^{-4}$ was introduced to each of the five magnets. The auxiliary $\beta$ functions at the end of the second solenoid were evaluated and compared to uncoupled $\beta$ functions see Fig. (54). Because the resulting changes in the auxiliary beta functions were small the insert was proven stable within the introduced magnet errors.

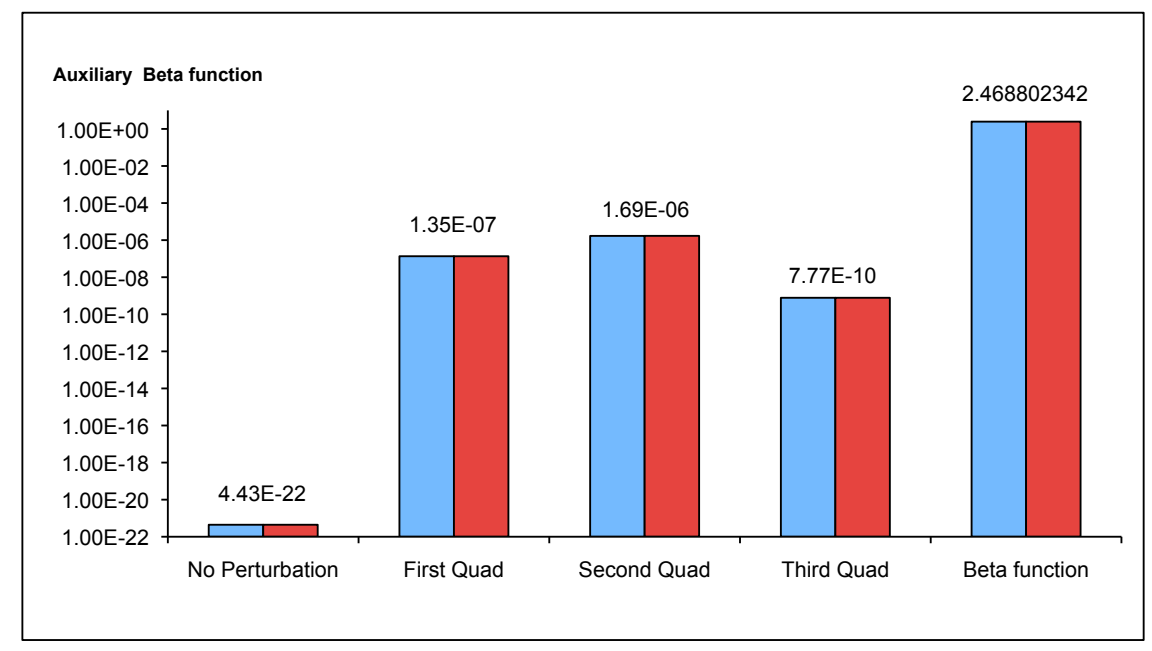

FIG. 54: Error analysis for MEIC coupling compensation insert.

\section{IV.9 RESULTS AND DISCUSSION}

In this chapter, an overall description for MEIC polarization manipulation was presented. An orbit decoupling insert has been designed to be implemented in a compact modular spin rotator. This insert works over the entire energy range by scaling the quadrupole field strength accordingly. It is short and compact enough to fit within the MEIC electron ring. The new orbit decoupling insert has a universal nature and can be implemented between any symmetric orbit coupling elements (solenoids, skew quadrupoles, ... etc). The orbit decoupling insert is independent of the coupling rotation angle. 


\section{CHAPTER V \\ ELECTRON ION COLLIDER ELIC}

The main differences between MEIC and ELIC are the design luminosity and energy. The design luminosity of MIEC is in the range of $10^{32}-10^{34} \mathrm{~cm}^{-2} \mathrm{sec}^{-1}$, while it is in the range of $10^{33}-10^{35} \mathrm{~cm}^{-2} \mathrm{sec}^{-1}$ for ELIC. Table (17) lists the main design parameters of ELIC and MEIC [6].

The lattice design of the electron ring of ELIC will presented in this chapter including the interaction region design. Later in the chapter the chromaticity correction scheme will be presented in detail.

TABLE 17: Design parameters for the MEIC and the ELIC.

\begin{tabular}{lccc}
\hline \hline Quantity & Unit & MEIC P/E & ELIC P/E \\
\hline Beam energy & $\mathrm{GeV}$ & $60 / 3-11$ & $30-225 / 3-9$ \\
Collision Frequency & $\mathrm{GHz}$ & 0.75 & 1.5 \\
Particles per bunch & $10^{10}$ & $0.416 / 2.5$ & $0.42 / 0.77$ \\
Beam current & $\mathrm{A}$ & $0.5 / 3$ & $1.0 / 1.85$ \\
Polarization & $\%$ & $70 / 80$ & $70 / 80$ \\
RMS bunch length & $\mathrm{mm}$ & $10 / 7.5$ & $5 / 5$ \\
Normalized horizontal emittance & $\mu \mathrm{m} \mathrm{rad}$ & $0.35 / 54$ & $1.25 / 90$ \\
Normalized vertical emittance & $\mu \mathrm{m} \mathrm{rad}$ & $0.07 / 11$ & $0.05 / 3.6$ \\
Horizontal $\beta^{*}$ & $\mathrm{~cm}$ & $10 / 10$ & $0.5 / 0.5$ \\
Vertical $\beta^{*}$ & $\mathrm{~cm}$ & $2 / 2$ & $0.5 / 0.5$ \\
Distance from IP to first FF quadrupole & $\mathrm{m}$ & $7 / 3.5$ & $3.8 / 3.8$ \\
Luminosity per IP & $\mathrm{cm}^{-2} \mathrm{sec}^{-1}$ & $10^{32}-10^{34}$ & $10^{33}-10^{35}$ \\
\hline \hline
\end{tabular}

\section{V.1 LATTICE DESIGN}

The lattice design of the electron ring of ELIC has to satisfy the same requirements as listed for the MEIC lattice in Chapter I.4. The basic layout of ELIC resembles that of MEIC. ELIC consists of three figure- 8 vertically stacked rings, two of them for the ion beam, while the third one is for the electron (or positron) beam. The figure-8 rings in ELIC have larger diameters than MEIC rings. The main components of ELIC's electron ring lattice are: 
1 Two identical arcs with opposite bending directions.

2 The first straight section contains.

- A dispersion matching section.

- Two spin rotators.

- An interaction region matching section.

- One interaction region (could be upgraded to four IRs, two in each straight section).

3 The second straight section contains.

- Two spin rotators.

- Dispersion adjusting section.

\section{V.1.1 Arc sections}

The ELIC figure-8 electron ring has two symmetric arc sections, arc I and arc II. The dipole magnets in arc II have the opposite polarity to those in arc I. Each arc section is composed of 84 FODO cells. Each arc cell is composed of two quadrupole magnets with alternating focusing and defocusing strength and two horizontally bending dipoles [13]. The magnet parameters are given in Table (18). One FODO cell magnetic lattice is shown in Fig. (55) along with their $\beta$-function and dispersion. The lattice parameters of the electron ring of ELIC are given in Table (19). Note especially the small $\beta^{*}$ at the IP of $5 \mathrm{~mm}$.

TABLE 18: Magnet parameters of an ELIC arc cell.

\begin{tabular}{lccc}
\hline \hline Magnet & $L[\mathrm{~m}]$ & $\theta[\mathrm{rad}]$ & $K\left[\mathrm{~m}^{-2}\right]$ \\
\hline QUADRUPOLE & 0.3 & 0.0 & 1.444 \\
QUADRUPOLE & 0.3 & 0.0 & -1.443 \\
DIPOLE ARC I & 3 & 0.027 & 0 \\
DIPOLE ARC II & 3 & -0.027 & 0 \\
\hline \hline
\end{tabular}

The total circumference of the figure- 8 ring is $2100 \mathrm{~m}$. The unit FODO cell has $120^{\circ}$ phase advance in both of the transverse planes, this phase advance per cell is 


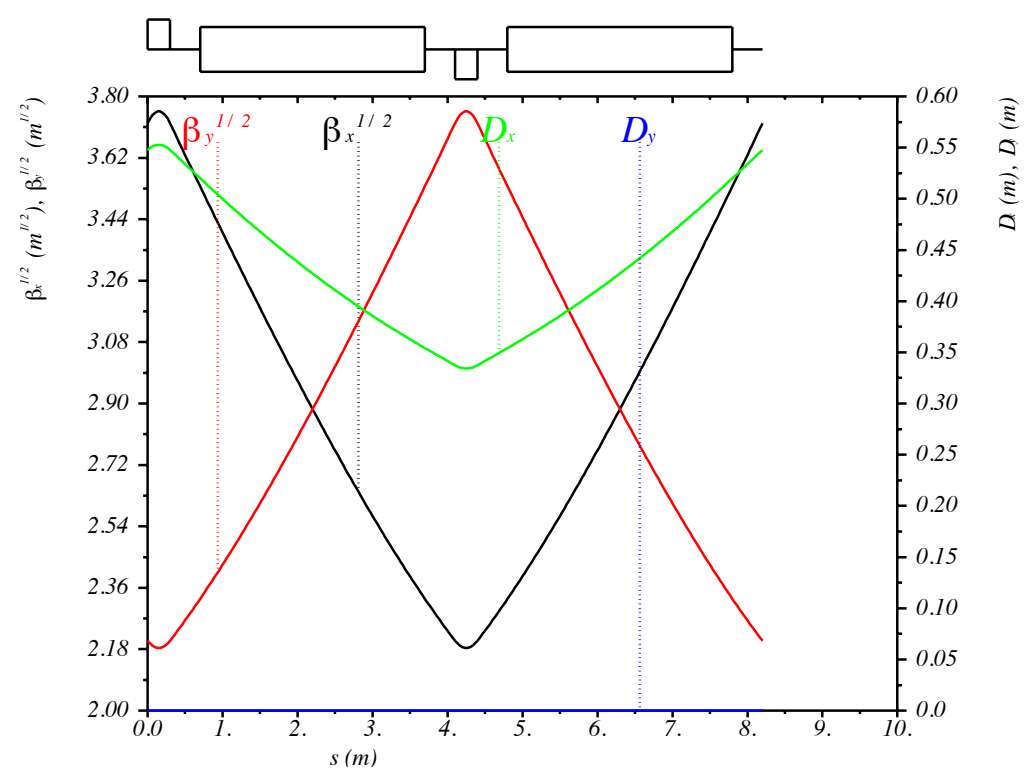

FIG. 55: Twiss function of ELIC arc cell

needed to obtain the required small equilibrium emittance. The dispersion function is well tailored to add chromatic correction sextupoles at places with large separation of the horizontal and vertical $\beta$ aspect ratio. Twiss functions $(\beta, \eta)$ for the entire figure-8 ring are illustrated in Fig. (60).

\section{V.1.2 Straight section}

The two straight sections are configured with the same phase advance per FODO cell as in the arc sections, plus a matching section, and the interaction region. The first straight section, straight section I, accommodates the interaction region and two spin rotators. The dispersion needed for the local chromaticity correction was generated by the arc dipoles and propagated by design into the interaction region while maintaining zero dispersion at the IP. The second straight section, straight section II, will accommodate the RF section and two spin rotators. The dispersion was suppressed in straight section II using the last 2 FODO cells of the arc section. Fig. (56) shows the last three arc cells suppressing dispersion in the beginning of straight section II. 
TABLE 19: Parameters of an ELIC lattice.

\begin{tabular}{lc}
\hline \hline Quantity & Value \\
\hline Beam energy $E$ & $9 \mathrm{GeV}$ \\
Particles/bunch $N$ & $0.77 \times 10^{10}$ \\
Bunch length $\sigma_{s}$ & $5 \times 10^{-3} \mathrm{~m}$ \\
Energy loss/turn $U_{0}$ & $7.42 \mathrm{MeV}$ \\
Horizontal $\beta_{x}^{*}$ & $0.005 \mathrm{~m}$ \\
Vertical $\beta_{y}^{*}$ & $0.005 \mathrm{~m}$ \\
Horizontal equilibrium emittance $\varepsilon_{x}$ & $5.6 \times 10^{-9} \mathrm{~m}$ \\
Momentum spread $\delta_{p}$ & $7.3 \times 10^{-4}$ \\
Momentum compaction $\alpha_{c}$ & $5.56 \times 10^{-4}$ \\
Horizontal damping time $\tau_{x}$ & $1.7 \times 10^{-2} \mathrm{sec}$ \\
Longitudinal damping time $\tau_{s}$ & $8.7 \times 10^{-3} \mathrm{sec}$ \\
\hline \hline
\end{tabular}

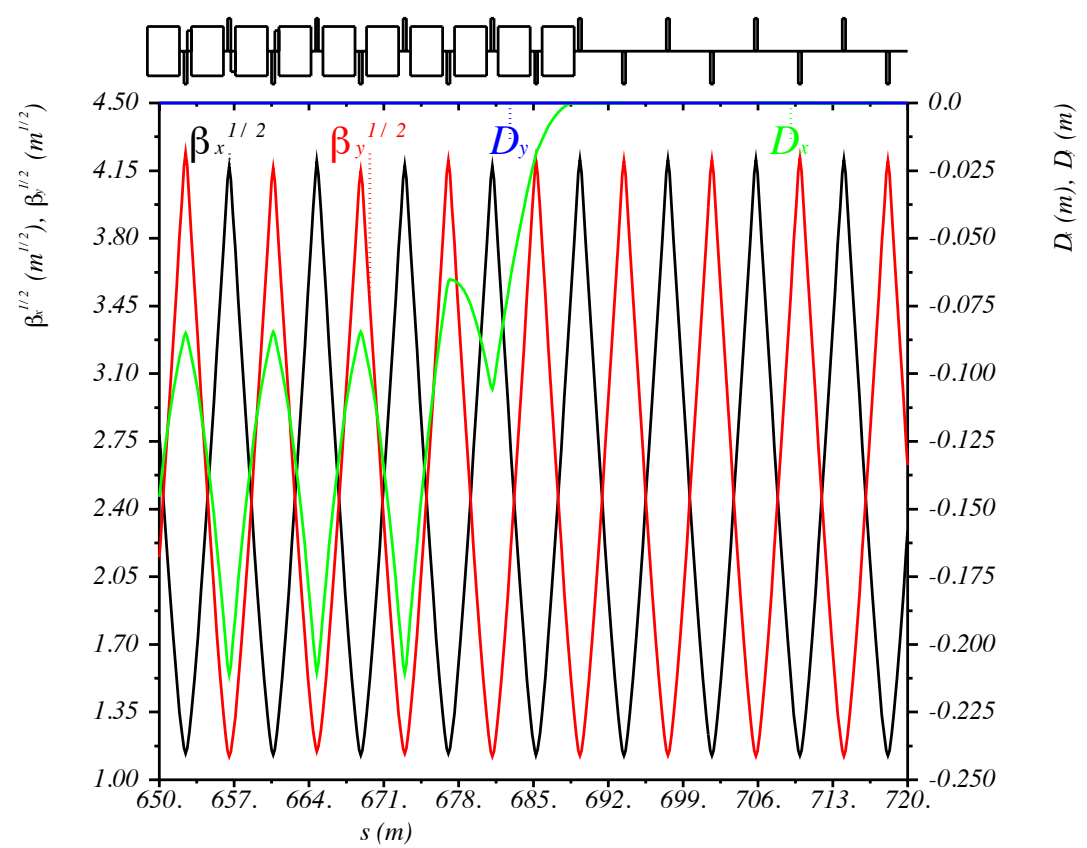

FIG. 56: Suppressing dispersion in the beginning of straight section II. 


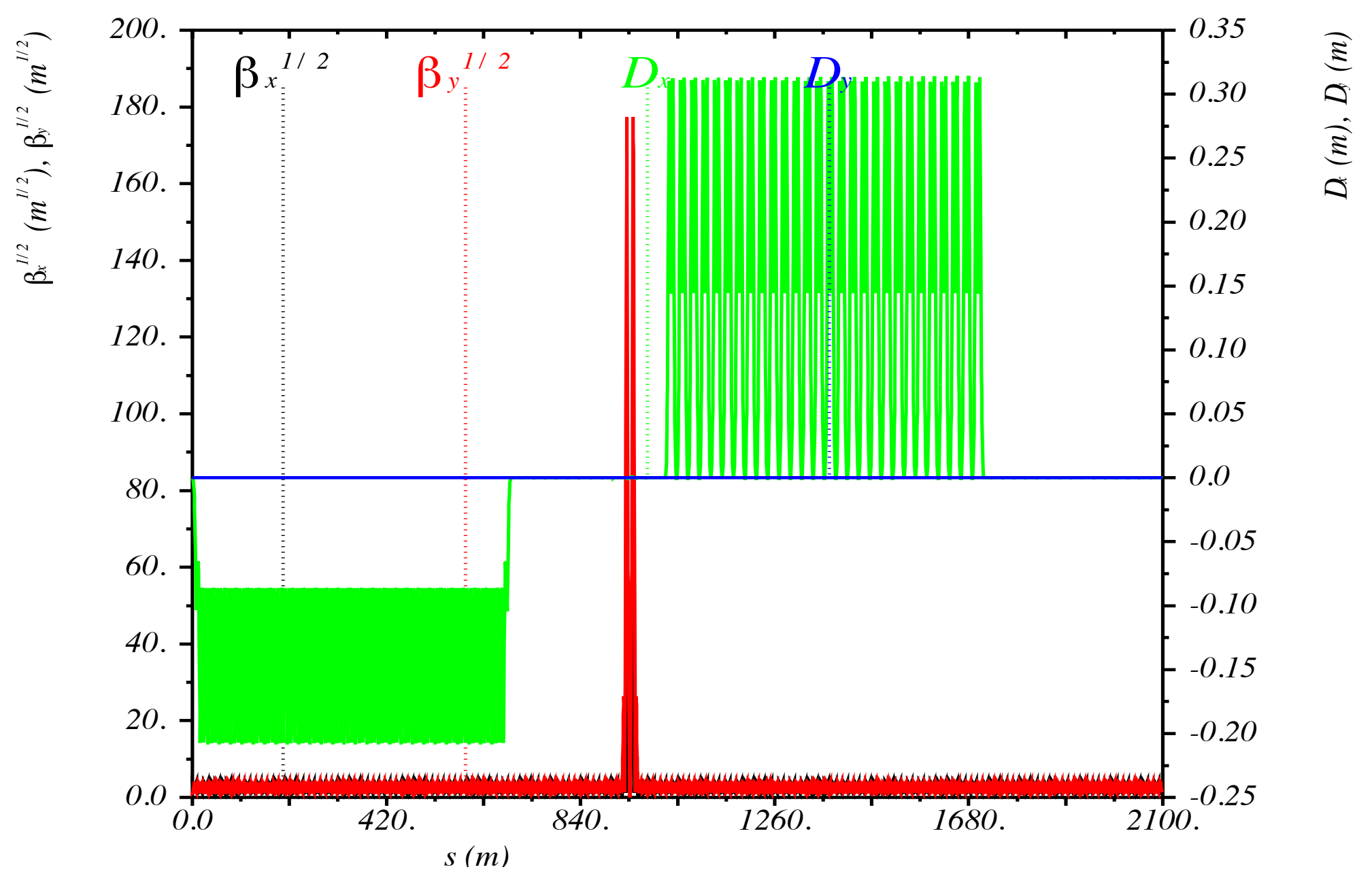

FIG. 57: Twiss functions of ELIC figure-8 ring. 


\section{V.1.3 The interaction region}

The electron beam interaction region is configured with two final focus quadrupole doublets, followed by the beam extension area, and a set of matching quadrupoles. The distance from the IP to final focus quadrupoles is set to $3.8 \mathrm{~m}$. The maximum $\beta$ function in the final focus quadrupoles is about $30 \mathrm{~km}$ in the vertical plane and 3 $\mathrm{km}$ in the horizontal plane. The interaction region is symmetric across the IP with antisymmetric dispersion. Fig. (58) shows the Twiss functions across the interaction region.

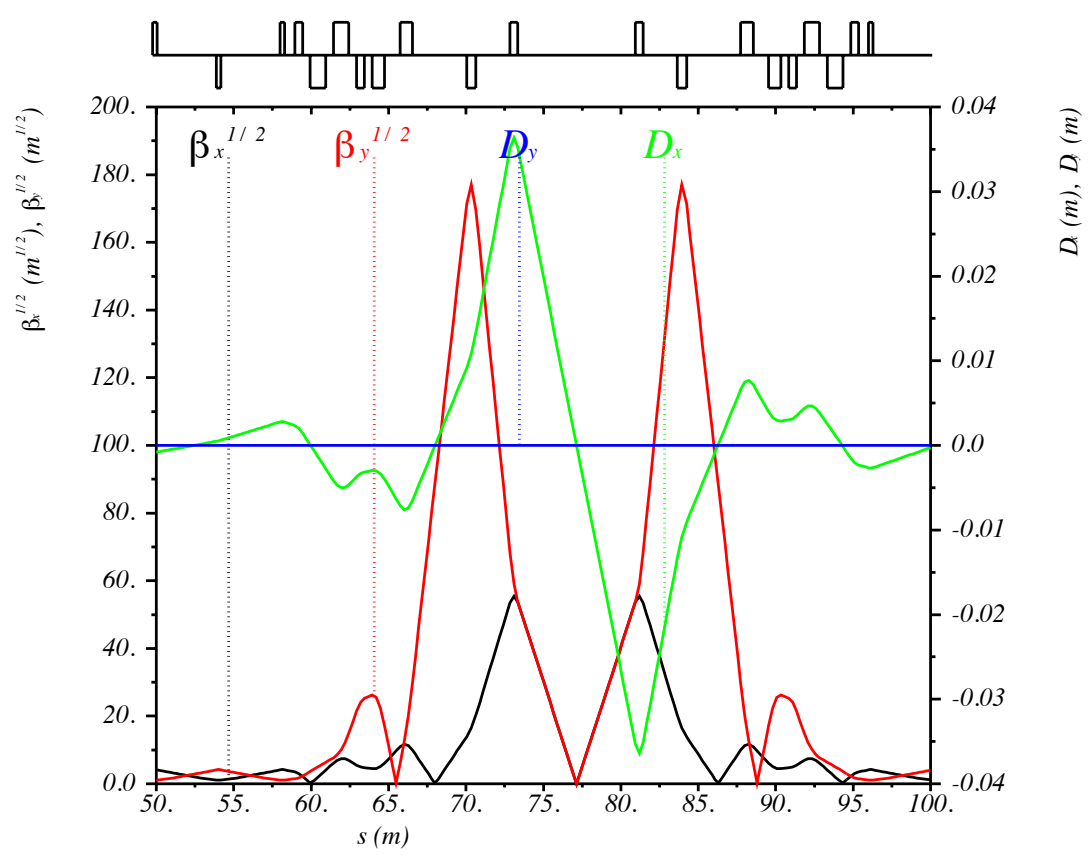

FIG. 58: Twiss functions in the interaction region. The dispersion function vanishes at the IP, while the derivative of dispersion does not vanish at IP.

The IR section consists of two final focus quadrupoles and two mitigating quadrupoles to focus the beam before it gets matched to the FODO cells by a matching section. The matching section is composed of four quadrupoles. Table (20) gives the magnet parameters of the IR. 
TABLE 20: Magnet parameters of the ELIC interaction region.

\begin{tabular}{lccc}
\hline \hline Lattice Element & $L[\mathrm{~m}]$ & $\theta[\mathrm{rad}]$ & $K\left[\mathrm{~m}^{-2}\right]$ \\
\hline Drift (IP to First Quad) & 3.8 & 0 & 0 \\
FF Quad I & 0.5 & 0.0 & 1.06586 \\
FF Quad II & 0.6 & 0.0 & -0.824 \\
Drift (Distance between Quad I \& II) & 2.2 & 0.0 & 0.0 \\
Drift (Beam Extension) & 3.5 & 0.0 & 0.0 \\
\hline \hline
\end{tabular}
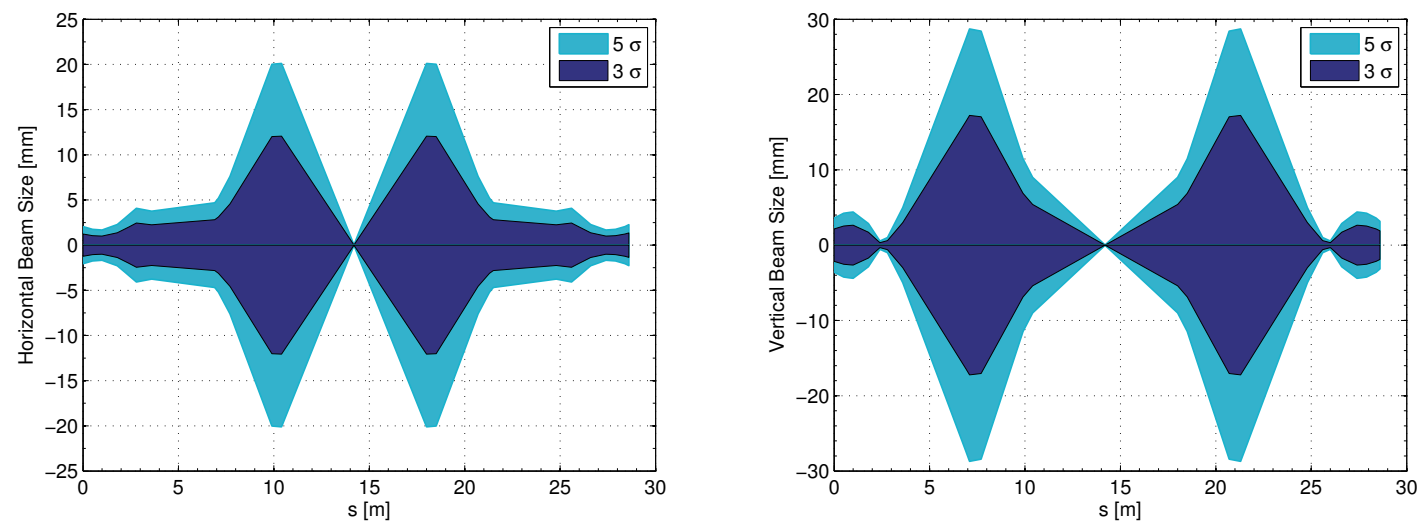

FIG. 59: Beam size at interaction region, the horizontal beam size at IP is $5.2 \times 10^{-5}$ $\mathrm{m}$ and $2.3 \times 10^{-6} \mathrm{~m}$ in the vertical, the maximum amplitude is $20 \mathrm{~mm}$ in horizontal and $29 \mathrm{~mm}$ in vertical. 


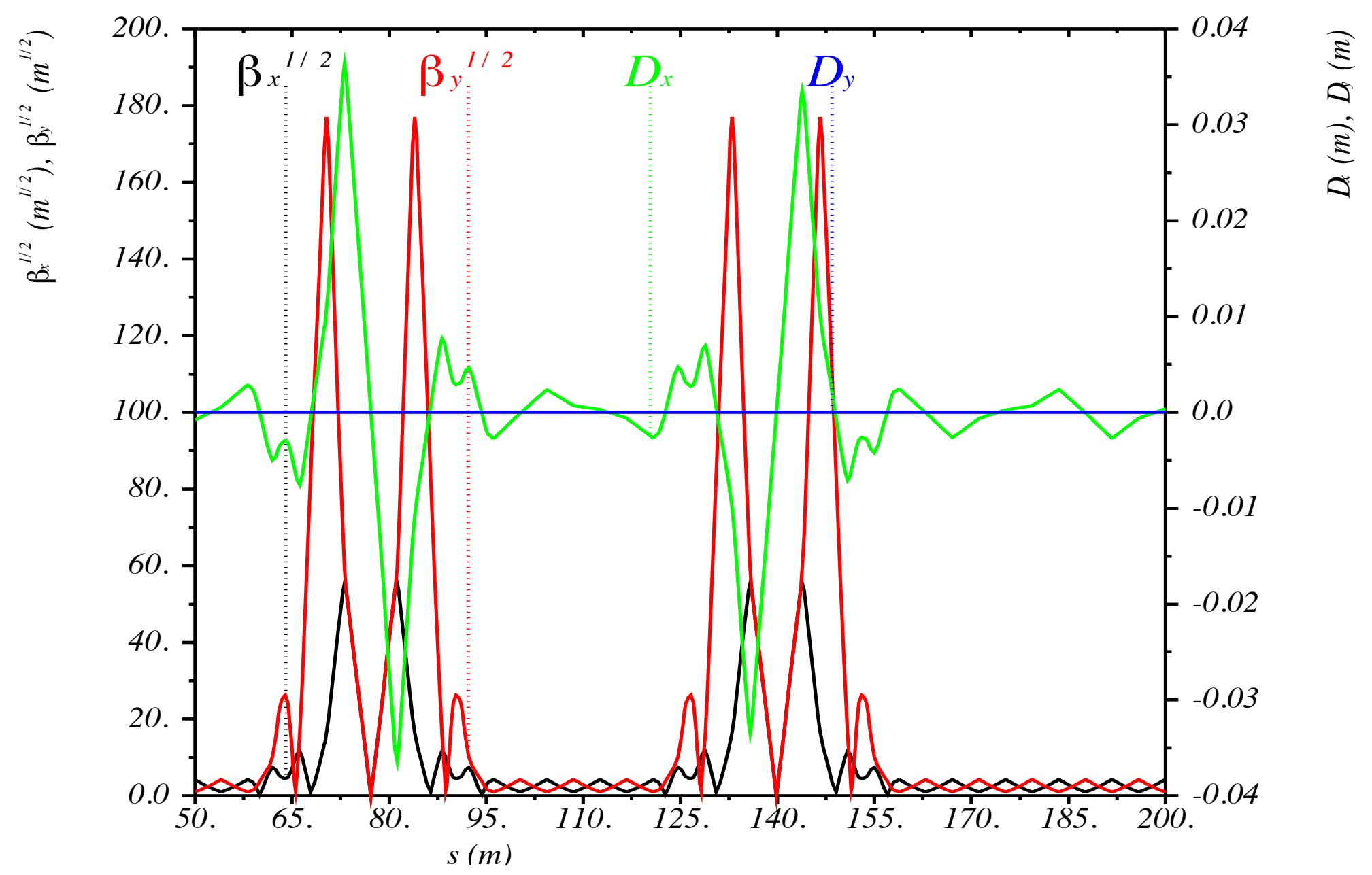

FIG. 60: Twiss functions of ELIC with two interaction regions on one straight section. 


\section{V.2 CHROMATICITY CORRECTION}

As in the MEIC case the final focus quadrupoles are the major sources of chromaticity. The extremely small $\beta$ function forces the maximum $\beta$ function in the final focus quadrupoles to extreme values (29481 $\mathrm{m}$ and $2889 \mathrm{~m}$ ). The huge $\beta$ function in final focus quadrupoles results in a large chromaticity.

\section{V.2.1 Local chromaticity correction}

Similarly to the MEIC antisymmetric IR design, the chromaticity correction in ELIC is implemented by two groups of sextupoles for local and global correcting. The local correction is carried out by a set of four sextupole families. Each family consists of two member sextupoles placed symmetrically across the IP. Each family member has a reversed polarity from its counterpart in the other side of the IP due to the reversed sign of the dispersion function across the IP. The dispersion needed for chromaticity correction is generated by the arc dipole magnets and shaped up by the IR matching quadrupoles to vanish at the IP. The Montague chromatic functions $W_{x, y}$ were employed to measure the $\beta$ chromaticity at the IP. Local correcting sextupoles around IP were used to reduce Montague functions from $10^{3}$ to around $10^{0}$ and they are confined to acceptable values throughout the rest of the ring. Fig. (61) shows Montague functions across the IR after the local chromatic correction. Table 21 lists the sextupole magnet parameters.

TABLE 21: Local correcting sextupole families.

\begin{tabular}{lccc}
\hline \hline Sextupole Family & Number of magnets & $L[\mathrm{~m}]$ & $M\left[\mathrm{~m}^{-3}\right]$ \\
\hline SIRF1 & 2 & 0.2 & 93463.4 \\
SIRD2 & 2 & 0.2 & -1.49574 \\
SIRD3 & 2 & 0.2 & -2154880 \\
SIRD4 & 2 & 0.2 & -0.0012 \\
\hline \hline
\end{tabular}

\section{V.2.2 Global chromaticity correction}

Two sextupole families were placed at each of the arc sections and they were used for chromatic correction; one family for the horizontal and one for the vertical. Sextupoles were placed in the arc cells adjacent to quadrupoles. To correct for tune 


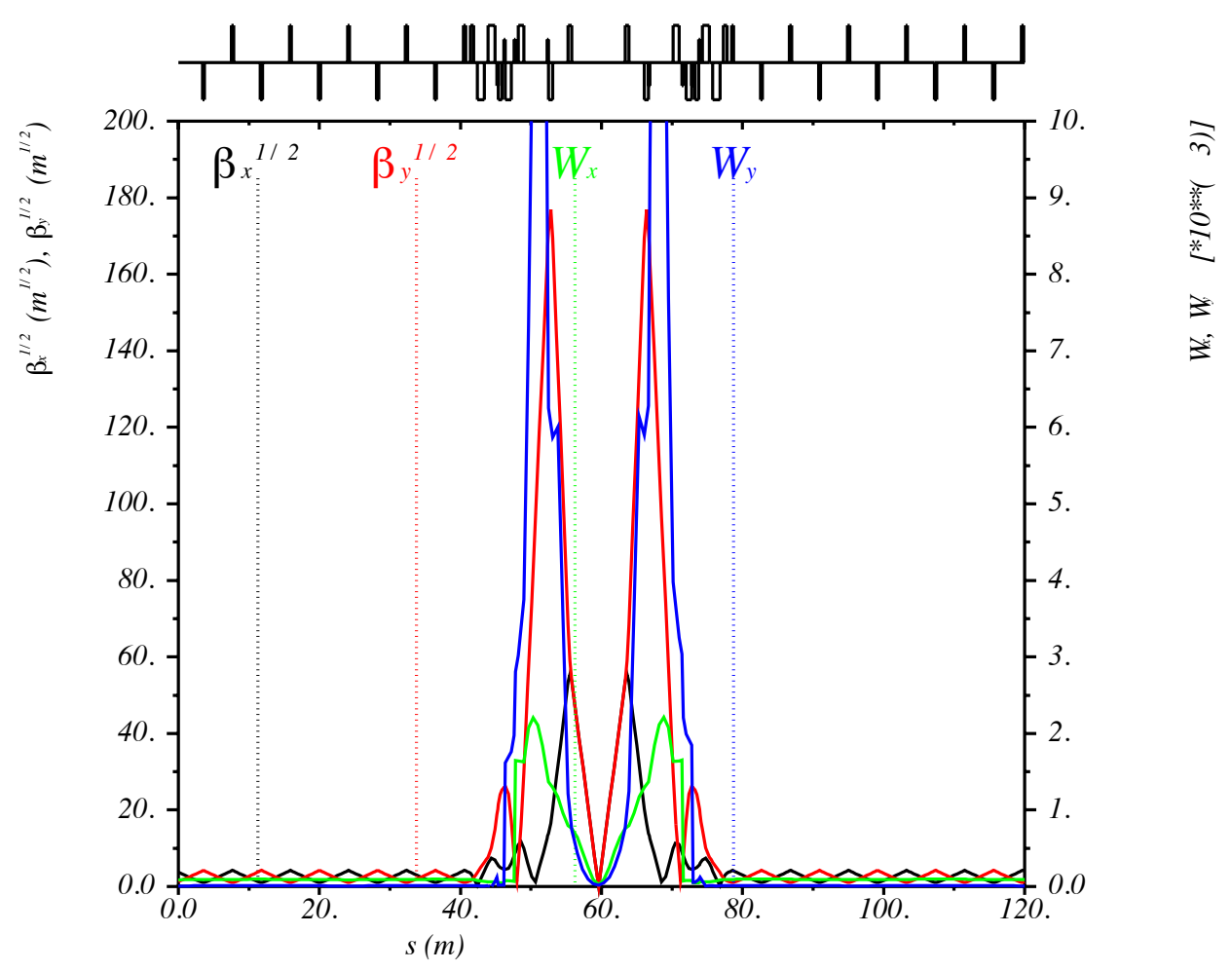

FIG. 61: Montague chromatic functions across ELIC IR after local chromatic correction using a set of 4 sextupole families.

chromaticity, sextupoles placed at the arcs were used for global tune correction. Several iterations involving the local correcting sextupole were performed. The correction was carried out using the computer code (MAD-X). During the optimization, sextupoles reserved for interaction region correction were fixed, while others were optimized to correct first and second order tune chromaticity. Fig. (62) shows tune variation with momentum deviation after correcting in both horizontal and vertical planes. The final momentum acceptance corresponds to 3 seconds of Touschek lifetime. 
TABLE 22: Global correcting sextupole families.

\begin{tabular}{lccc}
\hline \hline Sextupole Family & Number of magnets & $L[\mathrm{~m}]$ & $M\left[\mathrm{~m}^{-3}\right]$ \\
\hline SARCINF & 2 & 0.4 & -0.850888 \\
SARCOUTF & 2 & 0.4 & -80.2809 \\
SARCIND & 2 & 0.4 & 171.058 \\
SARCOUTD & 2 & 0.4 & 235.660 \\
\hline \hline
\end{tabular}

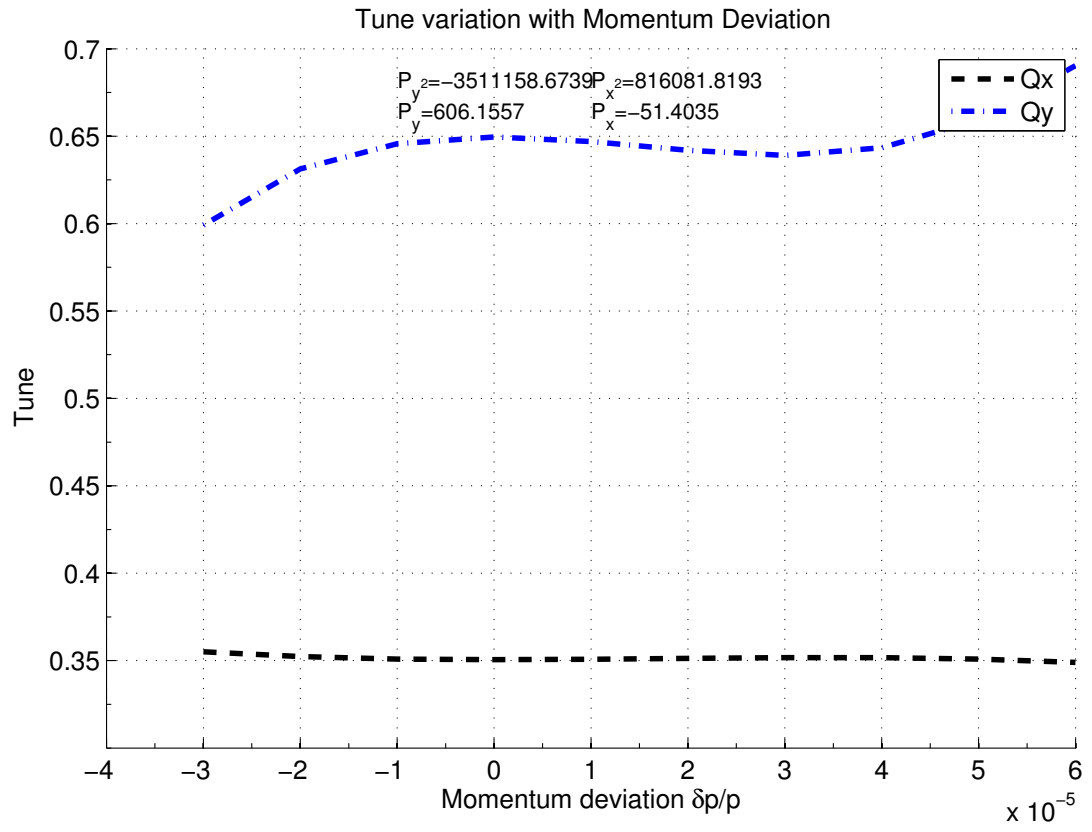

FIG. 62: Tune variation with momentum deviation for ELIC after correction. The momentum acceptance was limited to $3-6 \times 10^{-5}$ due to large initial chromaticity from the interaction region final focusing quadrupoles and very small $\beta^{*}$. 


\section{V.2.3 Touschek lifetime}

A calculation of the Touschek lifetime with the constant momentum acceptance $\delta_{\text {acc }}$ was performed for ELIC. The dependence of the Touschek lifetime on the constant momentum acceptance is showed in Fig. (63).
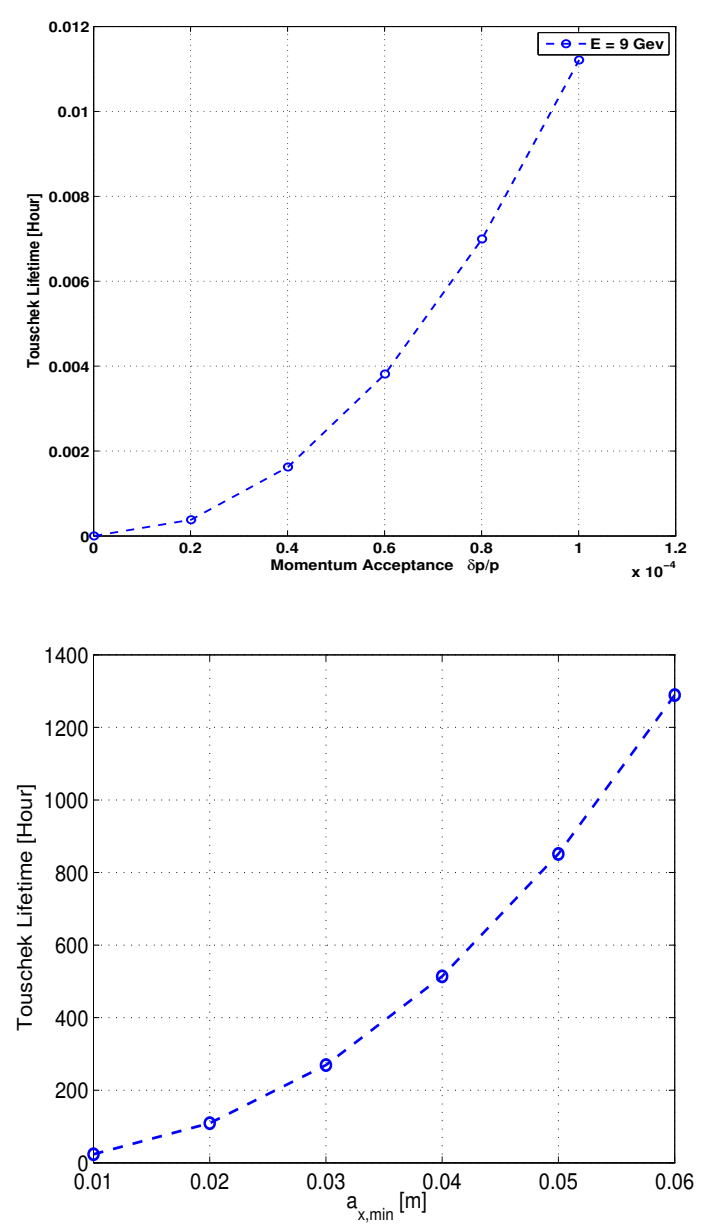

FIG. 63: Touschek lifetime for ELIC.

\section{V.3 SUMMARY}

ELIC is an upgrade of MEIC in terms of luminosity and the energy of the ion beam. The basic layout of ELIC was discussed in this chapter and the lattice structure was 
presented. The two arc sections were configured with FODO cells, each FODO cell has $120^{\circ}$ of betatron phase advance in both horizontal and vertical planes. The ELIC has two straight sections; the first straight section accommodates the interaction region, while the second straight section will be reserved for rf accelerating cavities.

The interaction region in the first straight is composed of two final focus quadrupoles and a matching section. The IR is symmetric around the IP with antisymmetric dispersion. The dispersion function in the IR was generated by the arc dipoles and suppressed by matching quadrupoles to vanish at the IP. The dispersion derivative in the IP has a finite value.

Chromaticity correction was done for ELIC lattice using local correcting sextupoles at the IR and global correcting sextupoles at the arcs. The very small $\beta$ * of $5 \mathrm{~mm}$ and the $3.5 \mathrm{~m}$ of magnet free space from IP to final focusing quadrupoles resulted in a substantial $\beta_{\max }$ inside the final focusing quadrupoles. The generated chromaticity from the IR turned out to be too large to correct using the local and global correction schemes. The momentum acceptance after chromatic correction was limited to $\pm 0.003 \%$ which yields a very short Touschek lifetime of 3.5 seconds. The dynamic aperture tracking showed a very restricted coordinate space area $10^{-5} \sigma_{x, y}$ much smaller than the initial particle's amplitude. This small dynamic aperture is attributed to the very strong sextupole magnets used for chromaticity correction. In order to obtain better compensation and better control of chromaticity, the requirement of a small $\beta$ function of $5 \mathrm{~mm}$ at the interaction point may have to be relaxed. 


\section{CHAPTER VI}

\section{CONCLUSIONS}

In this thesis numerical evaluations of the design of the electron ring for the MEIC have been performed. The requirements presented for the MEIC are summarized in the following: a proton energy of 30-60 GeV (15-30 GeV/A for ions) and an electron (or positron) energy of 3-11 GeV, a design luminosity between $10^{32}-10^{34} \mathrm{~cm}^{-2} \mathrm{sec}^{-1}$, support up to four collision points, support of simultaneous operation of the $12 \mathrm{GeV}$ CEBAF for fixed-target experiments, high polarization for both beams (up to 80\%), and finally the MEIC design has to support upgrade capability to higher energies in the ELIC (Electron Ion Collider).

The full design parameters of MEIC are given in Table (3). The main parameters presenting design difficulties solved in this thesis were the small $\beta^{*}$ functions of 2(10) $\mathrm{cm}$ in the vertical (horizontal) directions, a long magnet free distance from the IP to first final focusing quadrupole of $3.5 \mathrm{~m}$, a small normalized horizontal emittance of 54 $\mu \mathrm{m}$, and finally retaining a high polarization with a longitudinal polarization vector at the IP. The general layout of MEIC is composed of two vertically stacked figure8 collider rings intersecting at up to four collision points. The CEBAF accelerator serves as MEIC's electron (positron) full energy injector, while a whole injector chain is envisioned for the ion beam.

Only the electron ring design was considered in this thesis work. The basic components of the electron figure- 8 ring were: two identical arc sections with opposite bending directions and two straight sections connecting the two arc sections. Each of the straight sections accommodate an interaction region, the chromaticity compensation block, and two spin rotators. Each spin rotator includes a coupling compensation block.

The linear and non-linear equations of motion were derived from the Hamiltonian of a charged particle in electromagnetic field. Expressions for the first and second order tune chromaticity were derived from the equations of non-linear motion. An analytic calculation model was developed for the tune chromaticity generated by final focus quadrupoles in a straight section. Several chromaticity correction schemes were presented and evaluated. The methods explored are global chromaticity correction, local chromaticity correction, and adjusting the phase advance between low $\beta$ insertions to cancel second order chromaticity. The effect of limited momentum 
acceptance due to chromaticity on the Touschek lifetime was studied and quantified for the lattice designs considered. An efficient code for computing Touschek lifetime for a flat electron beam was developed and used in the computations.

Two different arc lattice designs were considered and the chromaticity generated by each was corrected and evaluated. The first arc lattice was constructed out of FODO cells, each cell having $135^{\circ}$ of phase advance. The second lattice has a $90^{\circ}$ phase advance per cell. Both designs produce a horizontal equilibrium emittance in the range of $4-6 \times 10^{-8} \mathrm{~m}$. A set of sextupole families were used in each case to correct natural chromaticity generated by the arc quadrupole magnets. The $90^{\circ}$ phase advance lattice showed better corrected tune chromaticity with a momentum acceptance of $\pm 2.0 \%$, and dynamic aperture of $120 \sigma_{x, y}$. The $135^{\circ}$ phase advance lattice proved less promising; it produced momentum acceptance of $\pm 0.8 \%$, and dynamic aperture of $85 \sigma_{x, y}$. We conclude that the $90^{\circ}$ phase advance lattice has more potential to use for electron collider rings in this application. The superior results of the $90^{\circ}$ phase advance lattice could be interpreted by the fact that cell quadrupoles are weaker and thus generate less chromaticity, and they require less sextupole strength to correct them.

The MEIC interaction region (IR) design is primarily driven by the detector needs for a very demanding forward detection architecture. The magnet free space between the IP and the first final focus quadrupole was set to $3.5 \mathrm{~m}$ for the electron beamline. Two IR designs were considered in this thesis, both have $2(10) \mathrm{cm}$ of $\beta_{y}^{*}\left(\beta_{x}^{*}\right)$, and the flat electron beam is focused by a set of doublets in both designs. The distinction between the two designs was the value of the dispersion function derivative at the IP. In the first case a non-vanishing dispersion derivative at the IP(antisymmetric case) is considered, and in the second a vanishing dispersion derivative case is considered (symmetric case). Tune chromaticity and local Montague chromatic function were corrected for both cases using local and global sextupoles. The antisymmetric IR design showed momentum acceptance sufficient for 3 hours of Touschek lifetime but the dynamic aperture was not large. On the other hand, the symmetric case showed higher correction of tune chromaticity, and a more promising dynamic aperture of 1.5-2.5 $\sigma_{x, y}$ with estimated Touschek lifetime of 55 hours. The symmetric IR design showed superior results which is explained by the fact that the sextupole families were symmetric across the IP (since dispersion is symmetric across IR), and the phase advance across the IR between symmetric sextupoles was $\pi$ which reduced 
second order effects from local correcting sextupoles.

The observed reduction of the dynamic aperture due to octupole magnets may require further study in future work. The study should include the effect of octupole magnets on dynamic aperture and optimizing the octupole magnet settings to produce larger dynamic aperture and small second order chromaticity. The MEIC rings studied in this thesis had either one or a maximum of two IRs. The MEIC ring with four IRs is expected to have much larger chromaticity. The chromaticity produced from four IRs may result in severe degradation of tune chromaticity, Touschek lifetime, and dynamic aperture of the lattice. A MEIC ring with four IRs may require increasing the $\beta$-functions at each of the IP in order to reduce the produced chromaticity. In summary, a study of similar MEIC lattices which include matching four IRs to the straight sections and evaluates the chromaticity may be required to fully understand the limit of correctable chromaticity for a given $\beta$ function.

An overall description of MEIC polarization manipulation was presented. A novel design for an orbit decoupling insert for a compact modular universal spin rotator (USR) was presented. The equations of coupled motion in the solenoid field were presented and used to compensate the coupling in the USR solenoid. The compensating insert works over a wide energy range by scaling the quadrupole field strength accordingly; it is short and compact enough to fit within the MEIC electron ring. Such compact decoupling inserts have a universal nature and can be implemented between any symmetric orbit coupling elements (solenoids, skew quadrupoles, ... etc), and have the additional feature that compensation is independent of the coupling rotation angle. The stability of the solution of such insert was evaluated and the solution was found to be stable within the recognized magnet errors. An example integrating the USR in the figure-8 lattice was presented.

The basic layout of ELIC was discussed and the lattice structure was presented. The two arc sections were configured with $120^{\circ}$ FODO cells. The IR is symmetric around the IP with antisymmetric dispersion. Chromaticity correction was done for ELIC lattice using local and global correcting sextupoles. The very small $\beta^{*}$ of $5 \mathrm{~mm}$ and the $3.5 \mathrm{~m}$ of magnet free space from IP to final focusing quadrupoles resulted in large chromaticity after local and global correction schemes. The momentum acceptance after chromatic correction was limited to $\pm 0.003 \%$ which yields a very short Touschek lifetime of 3.5 seconds. The dynamic aperture tracking showed a very restricted coordinate space area $10^{-5} \sigma_{x, y}$ much smaller than the initial particle's 
amplitude. This small dynamic aperture is attributed to the very strong sextupole magnets used for chromaticity correction. In order to obtain better compensation and better control of chromaticity, the requirement of a small $\beta$ function of $5 \mathrm{~mm}$ at the interaction point may have to be relaxed. 


\section{BIBLIOGRAPHY}

[1] G. Krafft etal.. MEIC Machine Design Status. JLAB-ACP-10-1225, 2010.

[2] A. W. Thomas. An electron-ion collider at Jefferson LAB. arXiv, hep-ex 0907.4785, 2009.

[3] A. Hutton. MEIC design parameters. CASA talk JLAB, 2010.

[4] A. Hutton. Low energy ring issues. SLAC-PUB-5807, 1992.

[5] A. Afanasev etal.. Zeroth Order Design Report for the Electron-Ion Collider at CEBAF. http ://casa.jlab.org/research/elic/elic_zdr.doc, 2007.

[6] A. Bogacz, P. Chevtsov, Y. Derbenev, P. Evtushenko, A. Hutton, G. Krafft, R. Li, L. Merminga, J. Musson, J. Qiang, H. Sayed, B. Yunn, and Y. Zhang. Advances on ELIC design studies. JLAB-ACC-08-826, 2008.

[7] G. Hoffstaetter, M. Vogt, and F. Willeke. Experiences with the HERA beams. ICFA Newsletter, pages 7-13, 2003.

[8] L. Cardman and L. Harwood. The JLAB 12 GeV energy upgrade of CEBAF for QCD and hadronic physics. Particle Accelerator Conference, 200\%. PAC. IEEE, pages 58-62, 2007.

[9] G. Voss and B. Wiik. The electron-proton collider HERA. Annual Review of Nuclear and Particle Science, 44:413-452, 1994.

[10] U. Schneekloth. Recent HERA results and future prospects. arXiv:hepex/9806010, 1998.

[11] A. Sokolov and I. Ternov. On polarization and spin effects in the theory of synchrotron radiation. Sov. Phys. Dokl., 8:1203, 1964.

[12] Y. Derbenev, P. Chevtsov, A. Bogacz, G. Krafft, and Y. Zhang. Electron Spin Rotation And Matching Scheme For ELIC, A High-Luminosity Ring-Ring Electron-Ion Collider. JLAB-ACP-09-1082, 2008.

[13] A. Bogacz, R. Ent, T. Horn, C. Hyde, and P. Nadel-Turonski. ELIC integrated detector and interaction region. JLAB Pub. 118119, 2011. 
[14] M. Bona et al.. Superb: A high-luminosity asymmetric $e^{+} e^{-}$super flavor factory. conceptual design report. SLAC-R-856, 2007.

[15] E. Keil. Linear machine lattices. First International School of Particle Accelerators "Ettore Majorana", 1976.

[16] E. Courant and H. Snyder. Theory of the alternating-gradient synchrotron. Annals of Physics, 3:1-48, 1958.

[17] J Bengtsson. The Sextupole Scheme for the Swiss Light Source (SLS): An Analytic Approach. SLS Note 9/97, 1997.

[18] John D. Jackson. Classical Electrodynamics. J.Wiley and sons, New York, third edition, 1998.

[19] M. Takao, H. Tanaka, and K. Soutome. Perturbative formulation for nonlinear chromaticity of circular accelerators. Physical Review E, 70:016501, 2004.

[20] H. Tanaka, M. Takao, K. Soutome, and H. Hama. A perturbative formulation of nonlinear dispersion for particle motion in storage rings. Nuclear Instruments and Methods in Physics Research, 431:396 - 408, 1999.

[21] K. Brown. Chromatic corrections for large storage rings. SLAC-PUB-2552 CONF-800740-15, 1980.

[22] T. Raubenheimer and F. Zimmermann. Final-focus systems in linear colliders. Rev. Mod. Phys., 72:95, 2000.

[23] B. Montague and S. Leger. Linear optics for improved chromaticity correction. CERN-LEP-Note-165. LEP-Note-165, 1979.

[24] P. Raimondi and A. Seryi. Novel final focus design for future linear colliders. Physical Review Letters, 86:3779-3782, 2001.

[25] K. Brown. Basic optics of the SLC final focus system. Proc. of the Workshop on Physics of Linear Collider, 296:481-494, 1988. 10.1007/BFb0031505.

[26] T. Sen, Y. Nosochkov, F. Pilat, R. Stiening, and D.M. Ritson. Chromaticity correction for the SSC collider rings. Proceedings of the Particle Accelerator Conference, 1:143-145, 1993. 
[27] S. Fartoukh. Large hadron collider project. LHC Project Report 308, 1999.

[28] J. Le Duff. Single and multiple Touschek effects. Advanced Accelerator Schools (CAS) Proceedings, 2:573-586, 1987.

[29] A. Streun. Momentum acceptance and Touschek lifetime. SLS Note 18/97, 1997.

[30] F. Zimmermann and M. Zorzano-Mier. Touschek scattering in HERA and LHC. LHC-PROJECT-NOTE-244, 2000.

[31] B. Terzic and Y. Zhang. Numerical simulation of beam-beam effects in the proposed electro-ion collider at Jefferson LAB. Proceedings of IPAC 2010, pages 1910-1912, 2010.

[32] T. Khoe, R. Kustom, R. Martin, E. Parker, C. Potts, L. Ratner, and R. Timm. High energy polarized beam at the ZGS. SLAC-REPRINT-1974-004, 1974.

[33] T. Roser, L. Ahrens, J. Alessi, and M. Bai. First polarized proton collisions at RHIC. AIP Conference Proceedings, 667:1-8, 2003.

[34] Y. Derbenev, A. Kondratenko, and A. Skrinskii. Dynamics of the Polarization of Particles Near Spin Resonances. Soviet Journal of Experimental and Theoretical Physics, 33:658, 1971.

[35] A. D. Krisch, S. R. Mane, R. S. Raymond, T. Roser, J. A. Stewart, K. M. Terwilliger, B. Vuaridel, J. E. Goodwin, H-O. Meyer, M. G. Minty, P. V. Pancella, R. E. Pollock, T. Rinckel, M. A. Ross, F. Sperisen, E. J. Stephenson, E. D. Courant, S. Y. Lee, and L. G. Ratner. First test of the siberian snake magnet arrangement to overcome depolarizing resonances in a circular accelerator. Phys. Rev. Lett., 63:1137-1140, 1989.

[36] M. Bai, L. Ahrens, J. Alessi, K. Brown, G. Bunce, P. Cameron, C. M. Chu, J. W. Glenn, H. Huang, A. E. Kponou, K. Krueger, W. Lamble, A. Luccio, Y. I. Makdisi, S. Y. Lee, M. Okamura, L. Ratner, K. Reece, T. Roser, H. Spinka, M. J. Syphers, N. Tsoupas, D. G. Underwood, W. Van Asselt, N. Williams, and A. Yokosawa. Overcoming intrinsic spin resonances with an RF dipole. Phys. Rev. Lett., 80:4673-4676, 1998. 
[37] T. Roser, L. Ahrens, J. Alessi, M. Bai, and J. Beebe-Wang. Accelerating and colliding polarized protons in RHIC with siberian snakes. Proc. of EPAC02, pages 209-211.

[38] T. Roser. RHIC operational status. Particle Accelerator Conference, pages 358 $-362,2006$.

[39] V. Bargmann and L. Michel. Precession of the polarization of particles moving in a homogeneous electromagnetic field. Phys. Rev. Lett., 2:435-436, 1959.

[40] H. Wiedemann. Particle accelerator physics. Springer, 2007.

[41] V. Lebedev and A. Bogacz. Betatron motion with coupling of horizontal and vertical degrees of freedom. Journal of Instrumentation, 5:10010, 2010.

[42] H. Sayed, A. Bogacz, and P. Chevtsov. Spin Rotator Optics for MEIC. Proceedings of 1st International Particle Accelerator Conference, pages 1626-1628, 2010.

[43] A. Zhelents and V. Litvinenko. On the compensation of solenoid field effects by quadrupole lenses. Preprint Novosibirsk Inst. Nucl. Phys. Acad. Sci., 1984.

[44] P. Emma. A spin rotator system for the NLC. NLC note \%, 1994.

[45] M. Borland. Elegant: A flexible SDDS-compliant code for accelerator simulation. ANL Pub. LS-287, 2000.

[46] F. Schmidt. MAD-X a worthy successor for MAD8. Nuclear Instruments and Methods in Physics Research, 558:47-49, 2006.

[47] F. Schmidt, Chao Yu Chiu, B. Goddard, D. Jacquet, V. Kain, M. Lamont, V. Mertens, J. Uythoven, and J. Wenninger. MAD-X PTC integration. Particle Accelerator Conference, page 4, 2005. 


\section{APPENDIX A}

\section{APPENDIX: BEAM DYNAMICS CODES}

\section{A.1 ELEGANT}

Elegant (Electron Generation And Tracking) [45] is an accelerator simulation code that includes 6-D tracking using matrices up to third order, canonical integration, and numerical integration. Supported beamline elements the were used in this thesis include but not limited to: solenoid, dipole, quadrupole, and sextupole.

Elegant has a range of capabilities including calculations of coherent synchrotron radiation, wakefields, rf elements, kickers, apertures, scattering, and tracking with and without errors. Elegant performs optimization of tracked properties, and computation and optimization of Twiss parameters, radiation integrals, matrices, and floor coordinates. Orbit/trajectory, tune, and chromaticity correction are supported. Elegant is fully compliant with the Self Describing Data Sets (SDDS) file protocol. Elegant lakes the calculations of Montague chromatic functions which proved to be crucial for chromaticity correction and optimization.

\section{A.2 MAD-X}

MAD-X [46] is a program for accelerator design and simulation which has an extension interface to PTC, the Polymorphic Tracking Code [47]. MAD-X is based on several languages: C, Fortran77, and Fortran90; with dynamic memory allocation (core program written in $\mathrm{C}$ ). MAD-X has a strictly modular organization. It supports symplectic and arbitrarily exact descriptions of all elements via PTC, Taylor Maps, and Normal Form techniques using PTC.

MAD-X proved to be especially useful for interaction region design and optimization, as well as chromaticity correction. It is able to calculate Montague chromatic functions. Several beamline elements are supported and were used in the work in this thesis: solenoid, dipole, quadrupole, sextupole, and octupole.

A simple comparison study was carried during this work between Elegant and MAD-X calculations. They came to excellent agreement in regard to Twiss functions and chromaticity calculations.

Chromaticity calculations are typically of the order of and agree with PTC and 
other codes. However, it was recently discovered that in presences of coupling, MAD$\mathrm{X}$ simply seems to ignore coupling when the chromaticity is calculated. 


\section{A.3 TOUSCHEK LIFETIME CODE}

Listing A.1: Touschek Lifetime Code

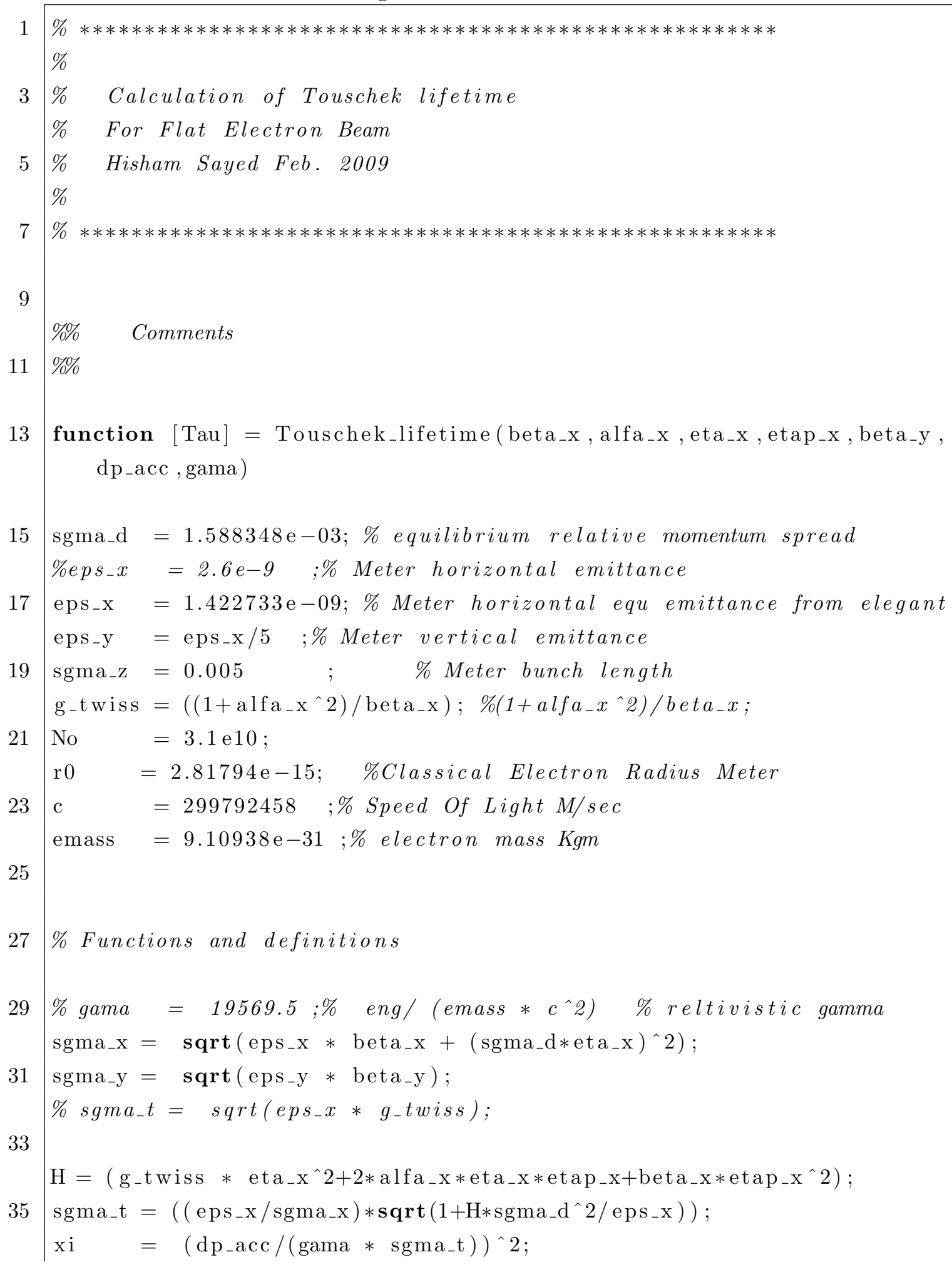




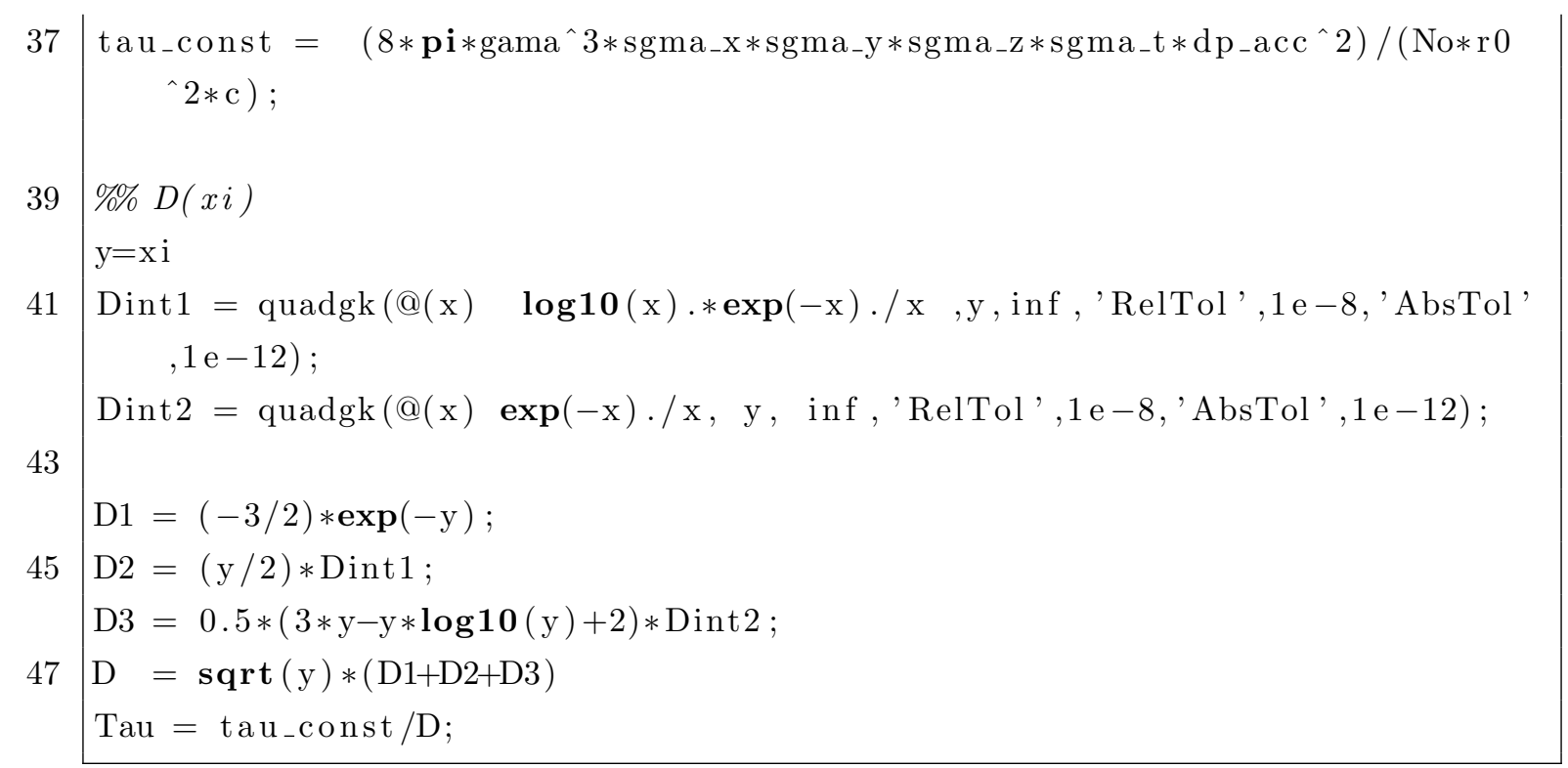

Listing A.2: Call Touschek Lifetime Code

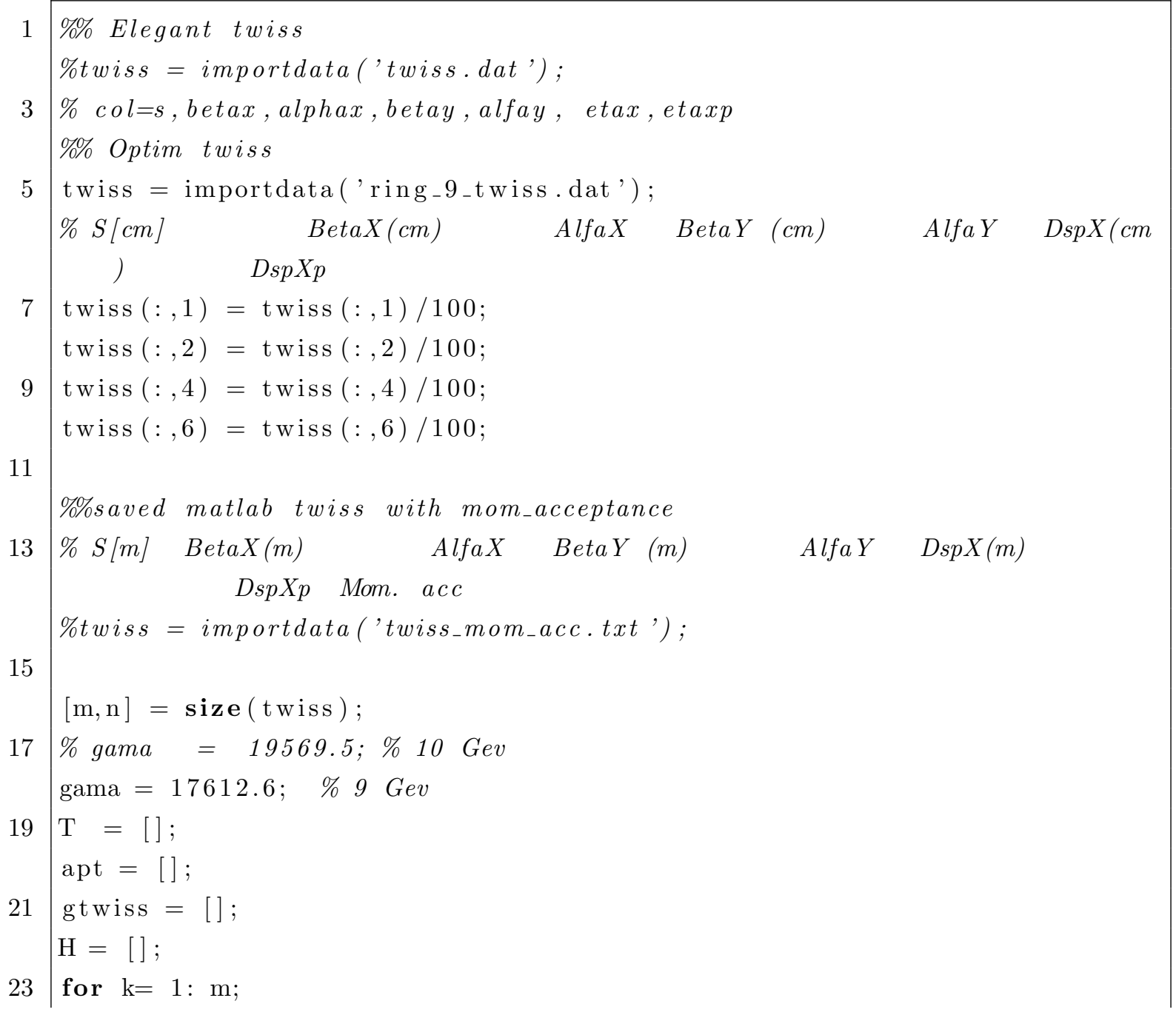




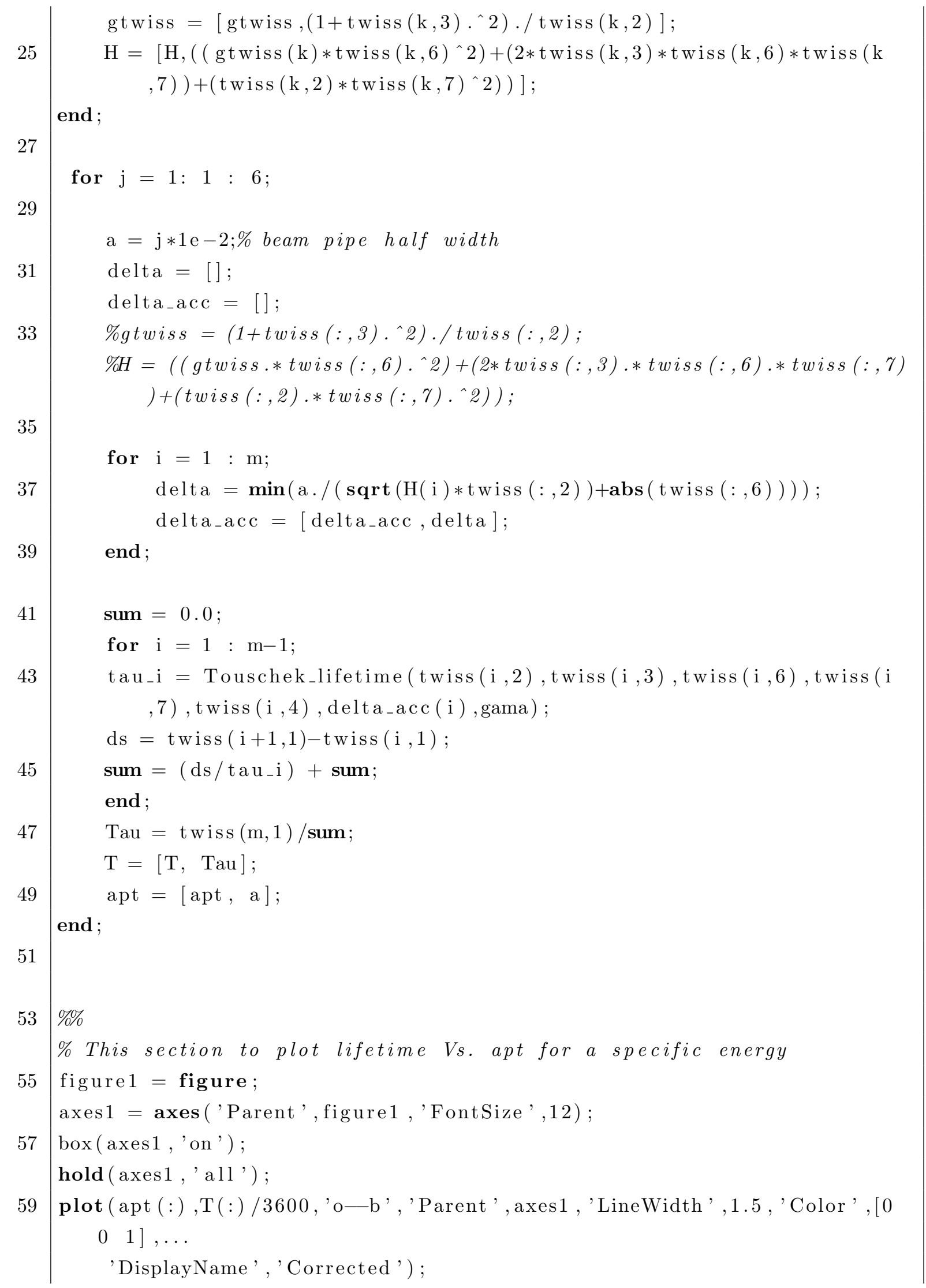


61 grid on;

xlabel (' $-a_{-}\{x, \min \}-[\mathrm{m}]$ ') ;

63 ylabel ('Touschek $\_$Lifetime $\leftarrow$ Hour ] ') ; 


\section{APPENDIX B}

\section{APPENDIX: MEIC LATTICES IN MAD-X FORMAT}

\section{B.1 MEIC $90^{\circ}$ LATTICES IN MAD-X FORMAT}

Listing B.1: MEIC 90 lattices in MAD-X format

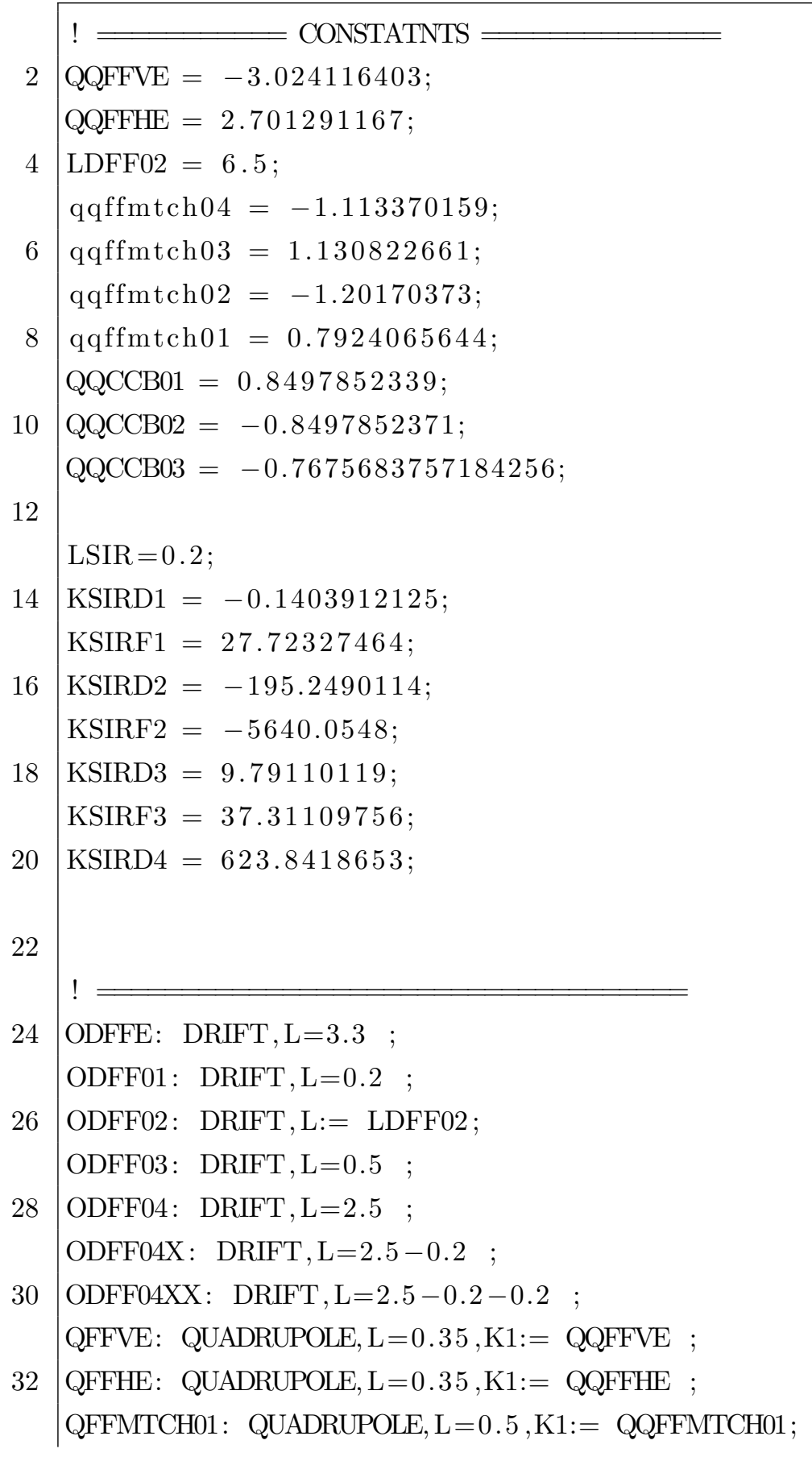


34 QFFMTCH02: QUADRUPOLE, $\mathrm{L}=0.5, \mathrm{~K} 1:=$ QQFFMTCH02;

QFFMTCH03: QUADRUPOLE, $\mathrm{L}=0.5, \mathrm{~K} 1:=$ QQFFMTCH03; QFFMTCH04: QUADRUPOLE, $\mathrm{L}=0.5, \mathrm{~K} 1:=$ QQFFMTCH04 ;

OCCB01: DRIFT, $\mathrm{L}=3$;

DCCBIN: SBEND, $\mathrm{L}=1.5$, ANGLE $:=\mathrm{DCCB} ; \quad !, \mathrm{E} 1:=\mathrm{DCCB} / 2, \mathrm{E} 2:=\mathrm{DCCB} / 2$, $\mathrm{HGAP}=0.1$

DFF1: SBEND, $\mathrm{L}=3, \mathrm{ANGLE}:=\mathrm{DCCB} 2 ; !, \mathrm{E} 1:=-\mathrm{DCCB} / 2, \mathrm{E} 2:=-\mathrm{DCCB} / 2$, $\mathrm{HGAP}=0.1 ;$ 


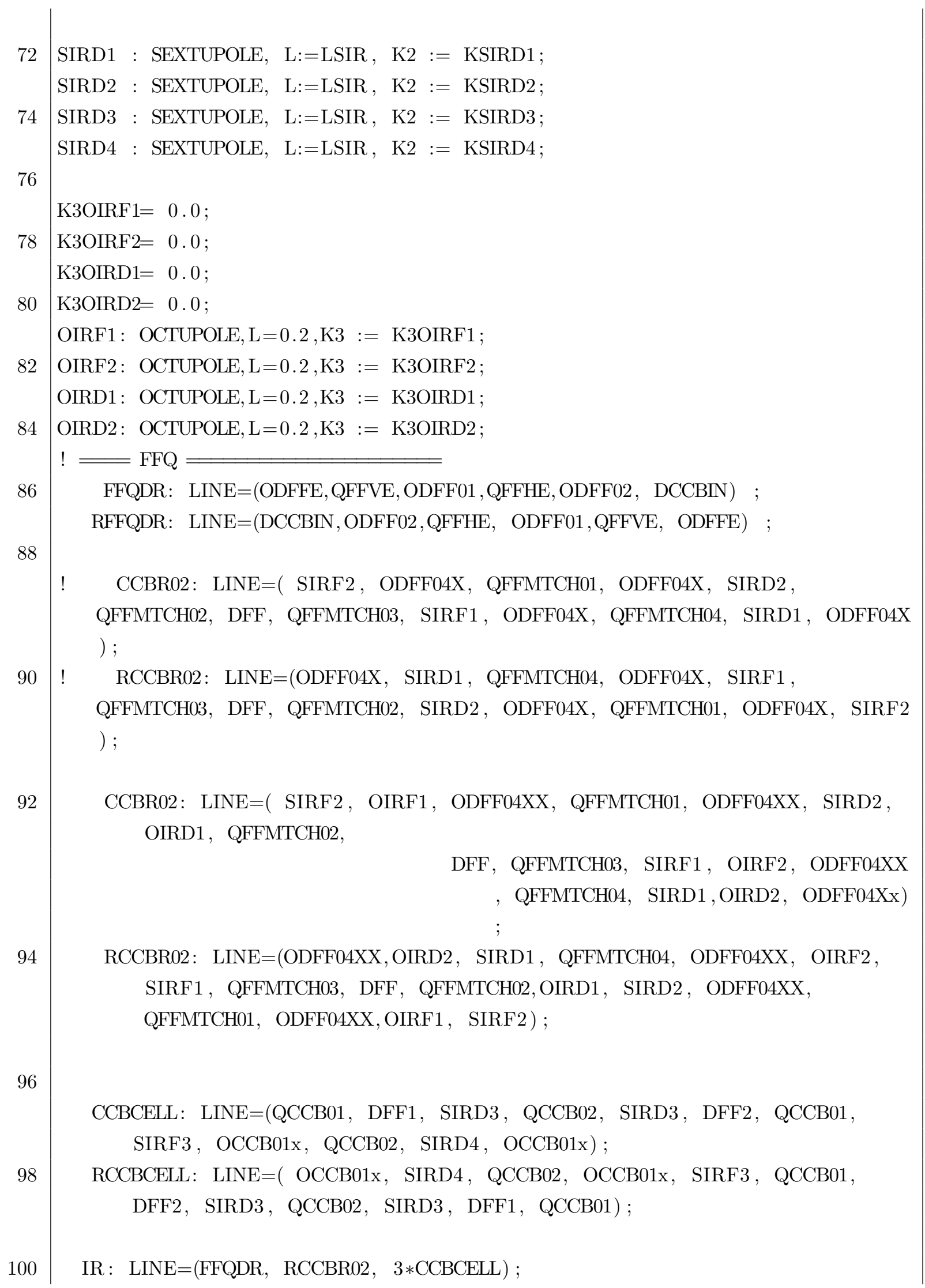




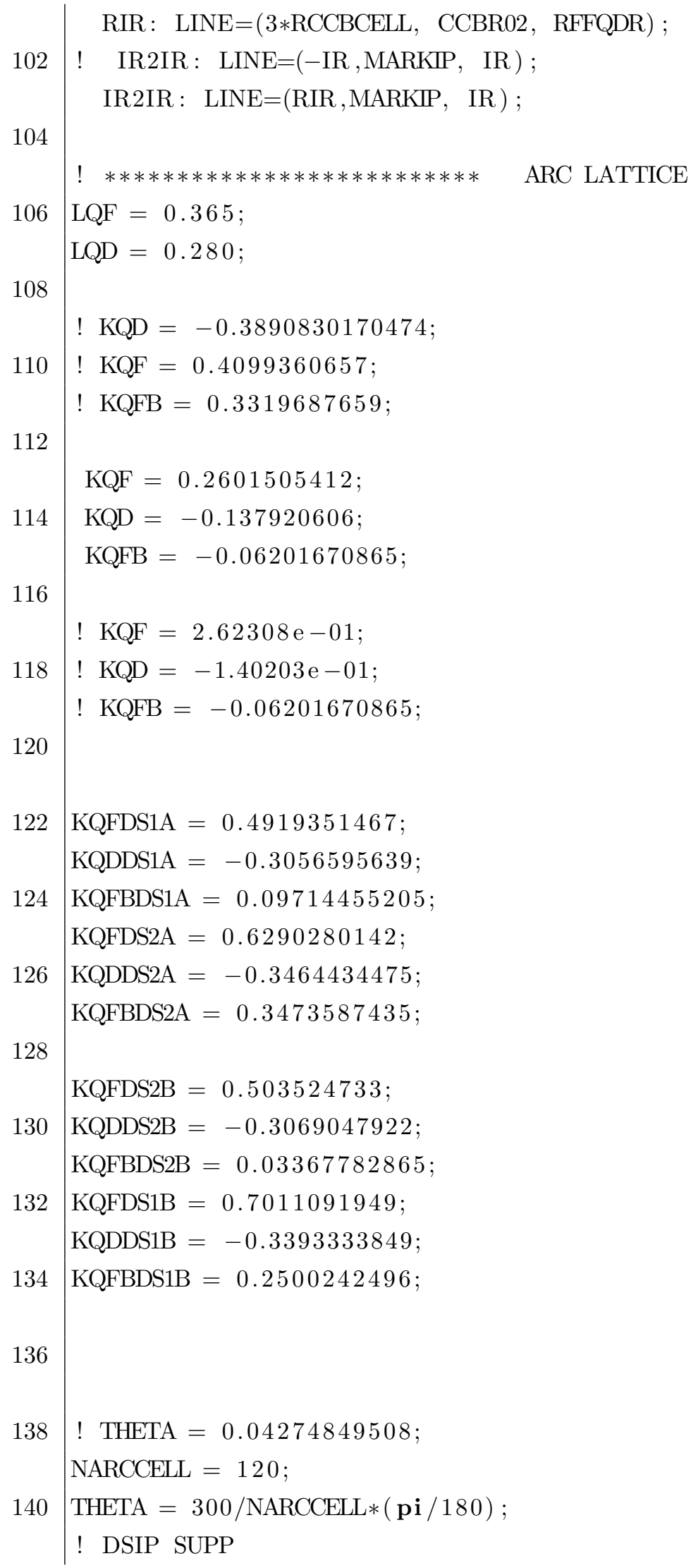




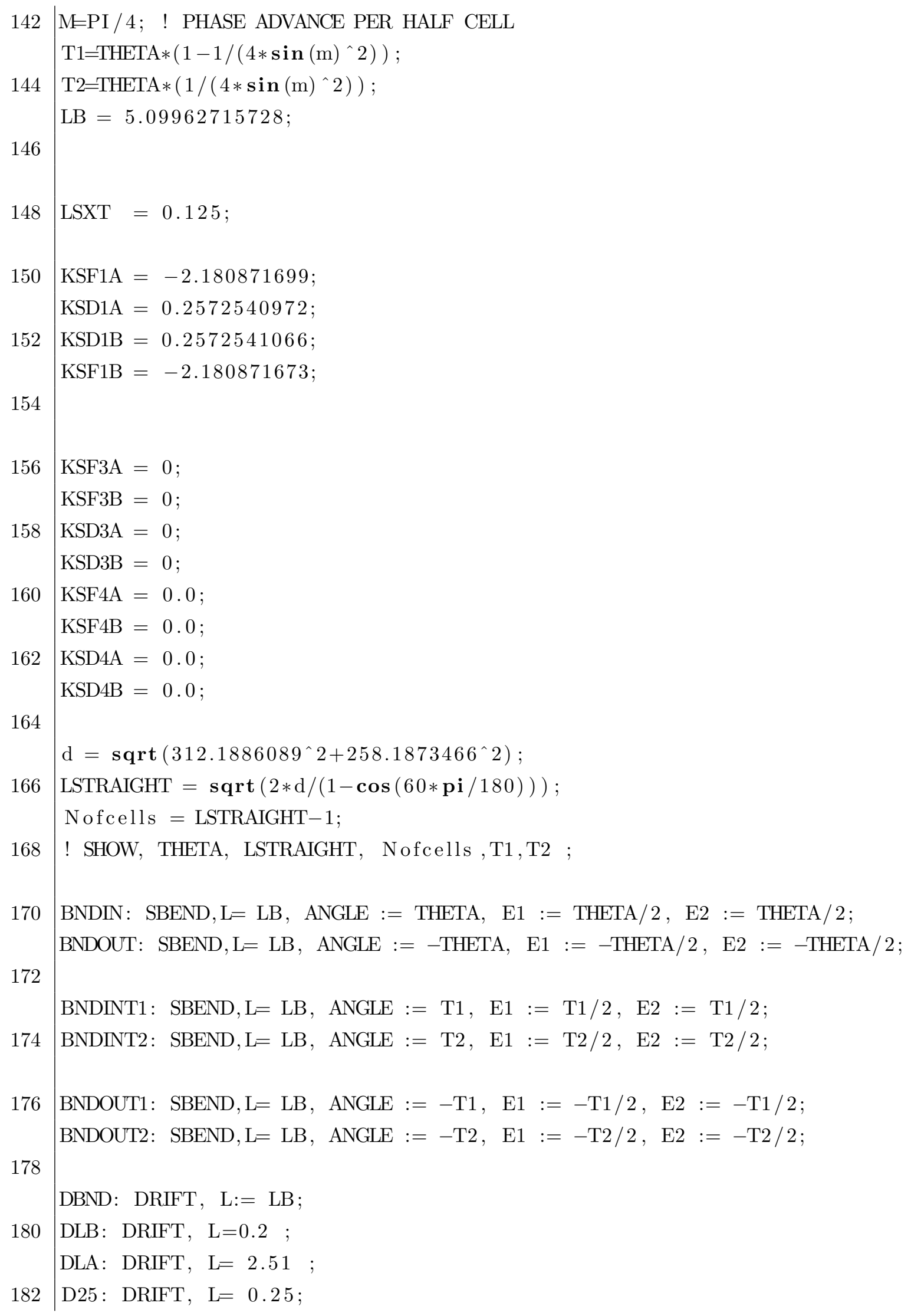







$224 \begin{aligned} & \text { SF4B : SEXTUPOLE, L:=LSXT, K2 }:=\text { KSF4B ; } \\ & \text { SD4B : SEXTUPOLE, L:=2*LSXT, K2 }:=\text { KSD4B ; } \\ & !=\text { SEXTS STRIAGHT } \\ & 228 \\ & \text { SF3A : SEXTUPOLE, L:=LSXT, K2 }:=- \text { KSF3A ; } \\ & \text { SD3A : SEXTUPOLE, L:=2*LSXT, K2 }:=\text { KSD3A ; } \\ & \text { SF3B : SEXTUPOLE, L:=LSXT, K2 }:=- \text { KSF3B ; } \\ & \text { SD3B : SEXTUPOLE, L:=2*LSXT, K2 }:=\text { KSD3B; }\end{aligned}$

CELLRA: LINE $=(\mathrm{QF}, \mathrm{SD} 1 \mathrm{~A}, \mathrm{DLB}, \mathrm{D} 25, \mathrm{DLA}, \mathrm{DLB}, \mathrm{QD}, \mathrm{DLB}, \mathrm{BNDIN}, \mathrm{DLB}, \mathrm{QFB})$

CELLRB: LINE $=(\mathrm{QF}, \mathrm{SD} 1 \mathrm{~B}, \mathrm{DLB}, \mathrm{D} 25, \mathrm{DLA}, \mathrm{DLB}, \mathrm{QD}, \mathrm{DLB}, \mathrm{BNDIN}, \mathrm{DLB}, \mathrm{QFB})$

RCELLRA： LINE=(QFB,DLB， BNDIN，DLB，QD，DLB，DLA，D25，DLB，SD1A，QF)

RCELLRB : LINE $=(\mathrm{QFB}, \mathrm{DLB}, \mathrm{BNDIN}, \mathrm{DLB}, \mathrm{QD}, \mathrm{DLB}, \mathrm{DLA}, \mathrm{D} 25, \mathrm{DLB}, \mathrm{SD} 1 \mathrm{~B}, \mathrm{QF})$ ;

! CELL_R : LINE=(CELL_RA，SF1A，RCELL_RB，CELL_RA，SF1B，RCELL_RB) ; CELL_R： LINE=(CELL_RA，SF1A，RCELL_RB，CELL_RA，SF1B，RCELL_RB) ; RCELL_R： LINE=(CELL_RB，SF1B，RCELL_RA，CELL_RB，SF1A， RCELL_RA） ;

CELL_LA: LINE $=(\mathrm{QF}, \mathrm{SD} 2 \mathrm{~A}, \mathrm{DLB}, \mathrm{D} 25, \mathrm{DLA}, \mathrm{DLB}, \mathrm{QD}, \mathrm{DLB}, \mathrm{BNDOUT}, \mathrm{DLB}, \mathrm{QFB})$

CELL_LB: LINE $=(\mathrm{QF}, \mathrm{SD} 2 \mathrm{~B}, \mathrm{DLB}, \mathrm{D} 25, \mathrm{DLA}, \mathrm{DLB}, \mathrm{QD}, \mathrm{DLB}, \mathrm{BNDOUT}, \mathrm{DLB}, \mathrm{QFB})$

RCELL_LA: LINE=(QFB,DLB，BNDOUT, DLB，QD，DLB，DLA，D25，DLB，SD2A，QF)

RCELLLB : LINE $=(\mathrm{QFB}, \mathrm{DLB}, \mathrm{BNDOUT}, \mathrm{DLB}, \mathrm{QD}, \mathrm{DLB}, \mathrm{DLA}, \mathrm{D} 25, \mathrm{DLB}, \mathrm{SD} 2 \mathrm{~B}, \mathrm{QF})$

! CELL_L: LINE=(CELlLA, SF2A, -CELLlB, CELLLA, SF2B, -CELL_LB) ; CELL_L: LINE=(CELLLA, SF2A, RCELLLB, CELLLA, SF2B， RCELLLB) ; RCELLL: LINE=(CELL_LB, SF2B， RCELLLA, CELL_LB, SF2A， RCELLLA ) ;

CELLMTCH: LINE=(DLA, QFMTCHA, DLB, DLA, QDMTCHA, DLB, DLA, QFBMTCHA, DLB, DLA, QFMTCHB, DLA);

RCELLMTCH: LINE=(DLA, QFMTCHB, DLA, DLB, QFBMTCHA, DLA, DLB, QDMTCHA , DLA, DLB, QFMTCHA, DLA); 


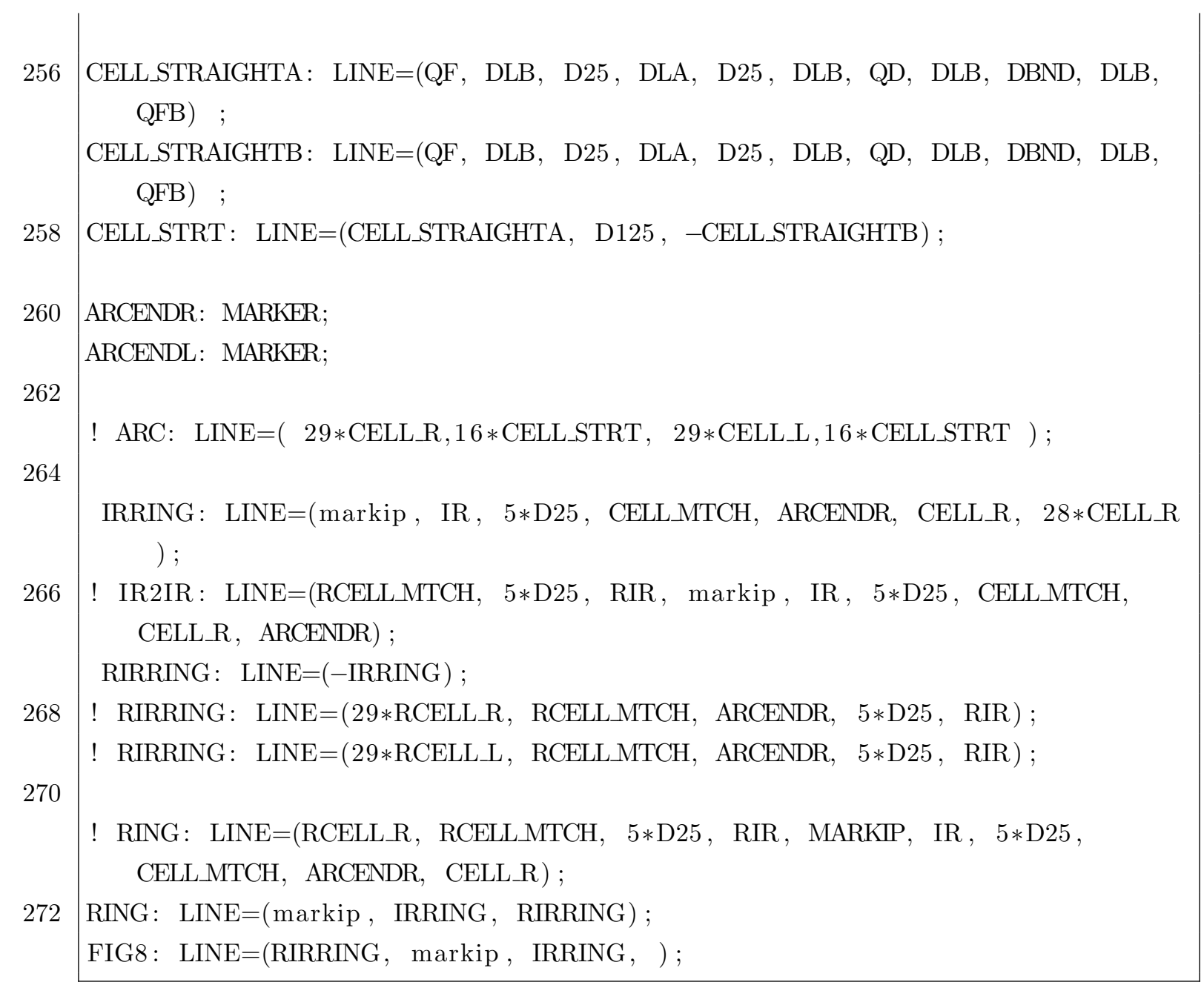

\section{B.2 MEIC $135^{\circ}$ LATTICES IN MAD-X FORMAT}

Listing B.2: MEIC 135 lattices in MAD-X format

1

qFF1: QUADRUPOLE, $\mathrm{L}=$ qFF2: QUADRUPOLE, $\quad \mathrm{L}=$

3

qQL1: QUADRUPOLE, L=

5 qQL2: QUADRUPOLE, $\mathrm{L}=$ qQL3: QUADRUPOLE, $\mathrm{L}=$

7 qFFR: QUADRUPOLE, $\mathrm{L}=$ qQR1: QUADRUPOLE, $\quad \mathrm{L}=$

9

11
qQR2: QUADRUPOLE, L= qQ6: QUADRUPOLE, L= qQ13: QUADRUPOLE, $\mathrm{L}=$ qQ14: QUADRUPOLE, L=
$0.5, \quad \mathrm{~K} 1=-1.710610246$;
$0.5, \quad \mathrm{~K} 1=1.793012185$;
$0.5, \quad \mathrm{~K} 1=-0.6980660777$;
$0.5, \quad \mathrm{~K} 1=0.4798035784$;
$0.5, \quad \mathrm{~K} 1=-0.001750974343$;
$0.5, \quad \mathrm{~K} 1=0.4330462711$;
$0.5, \quad \mathrm{~K} 1=-0.6980660777$;
$0.5, \quad \mathrm{~K} 1=0.5495883026$;
$0.5, \quad \mathrm{~K} 1=-0.1349845162$;
$0.5, \quad \mathrm{~K} 1=1.074875476$;
$0.5, \quad \mathrm{~K} 1=-0.1919464793$;
$0.5, \quad \mathrm{~K} 1=0.8166352573$; 


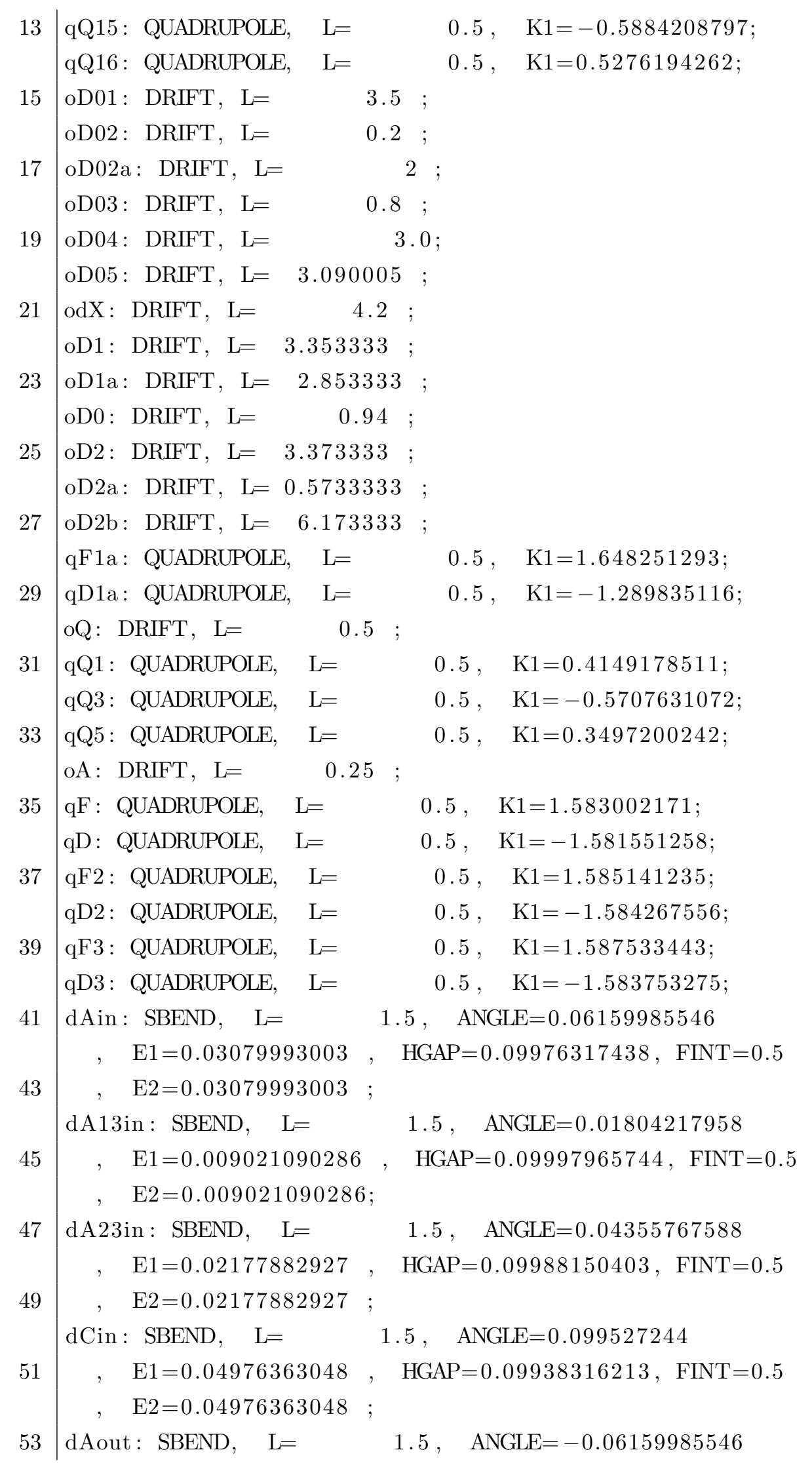




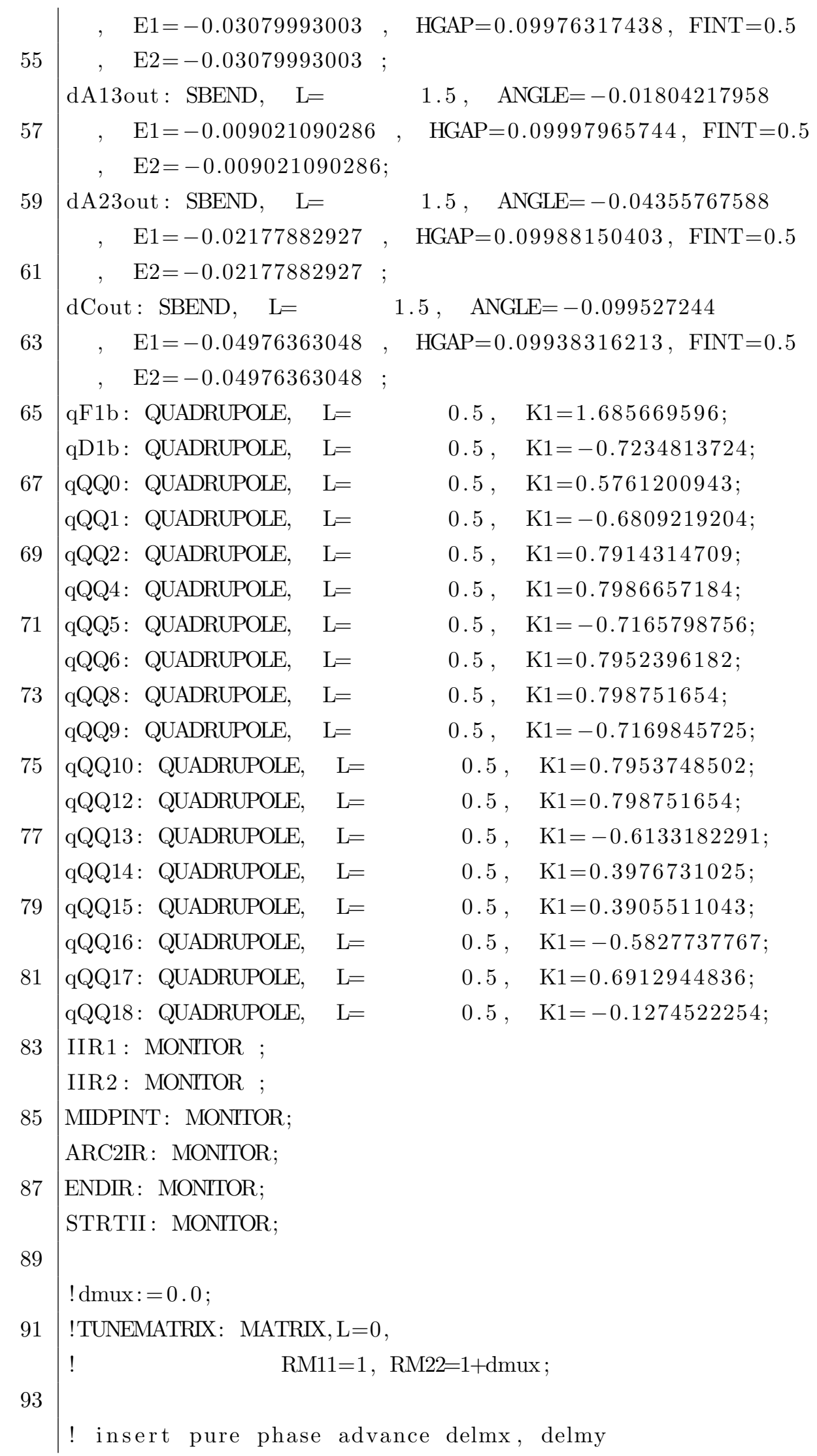




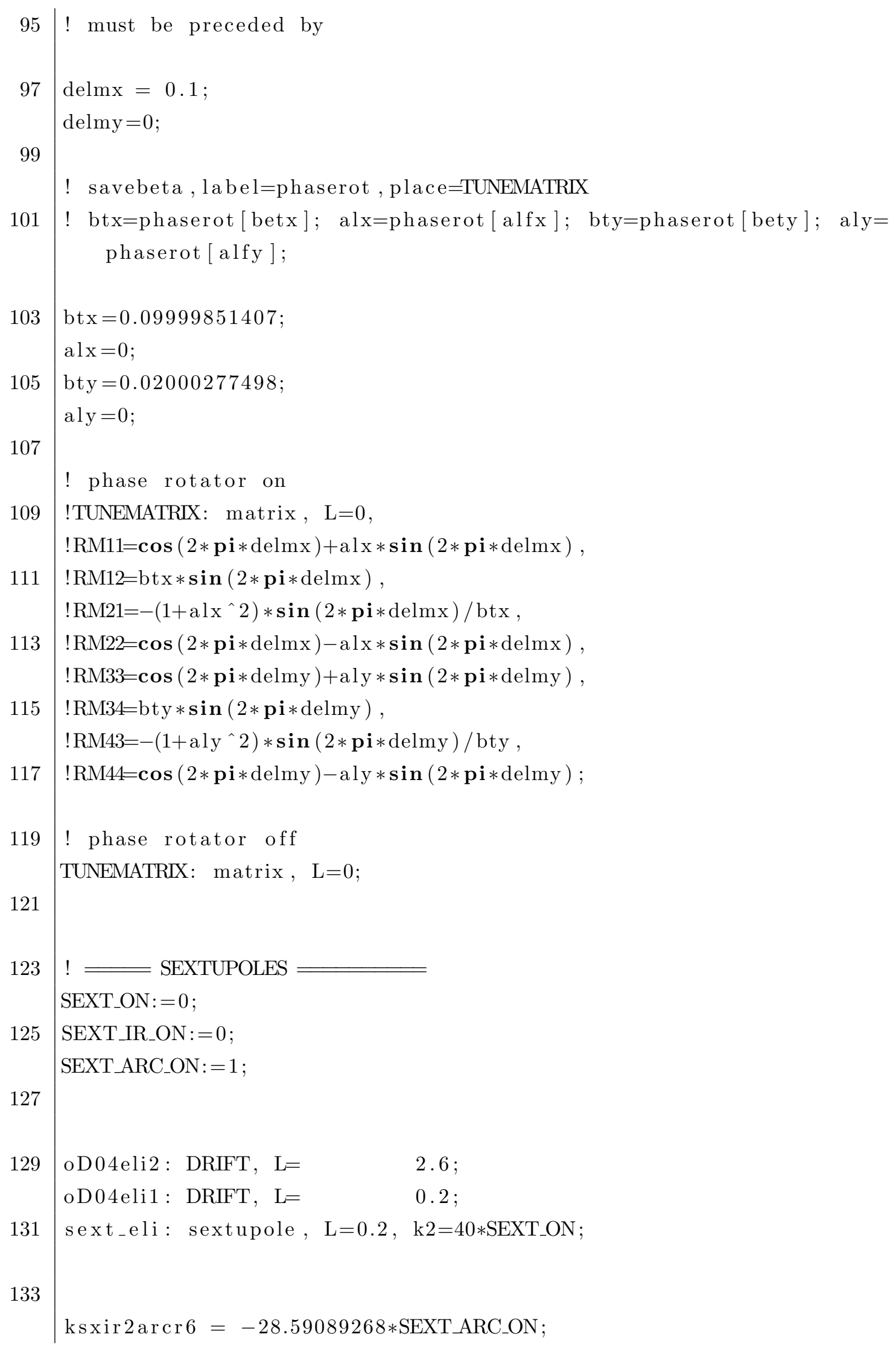




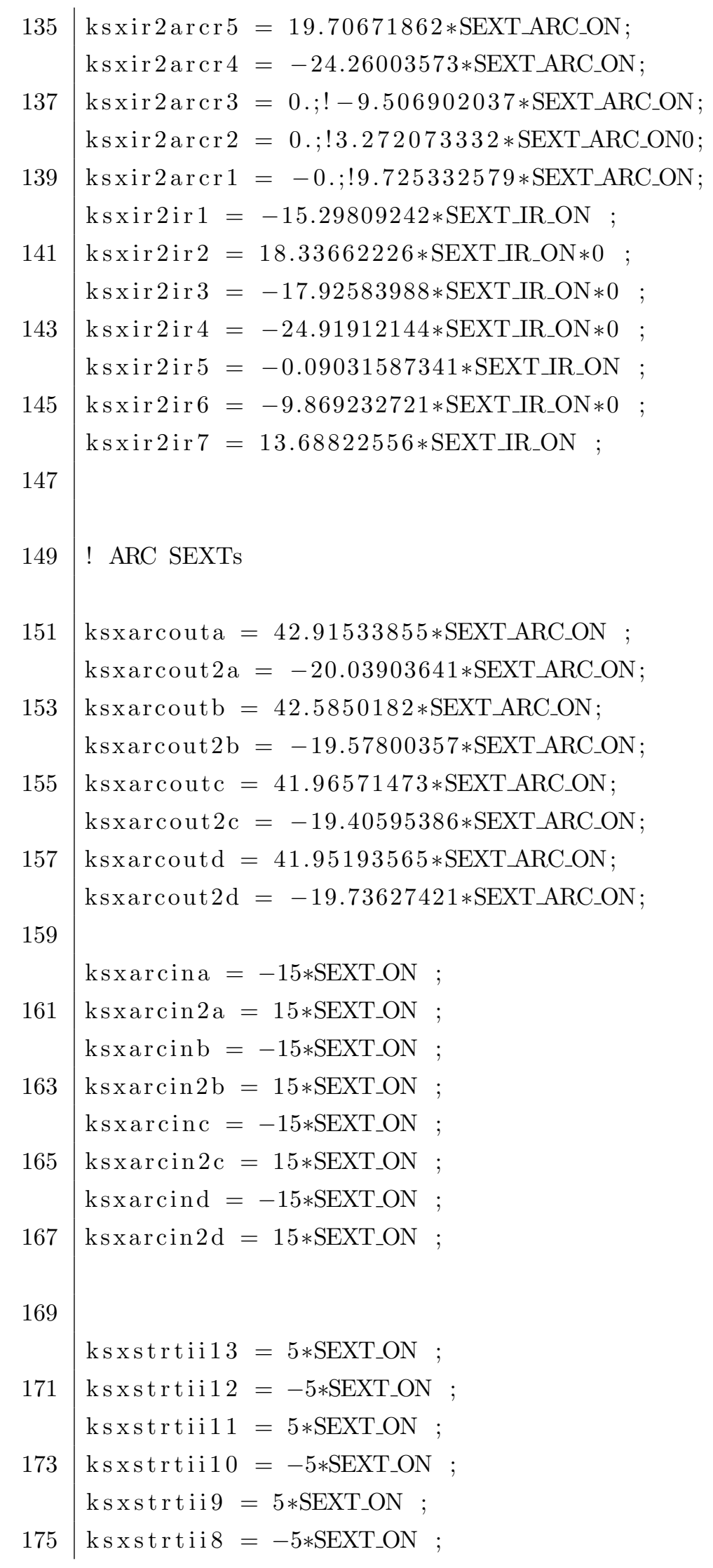




\begin{tabular}{|c|c|}
\hline \multirow{3}{*}{177} & ksxstrtii $7=5 *$ SEXT_ON \\
\hline & ksxstrtii $6=-5 *$ SEXT_ON \\
\hline & ksxstrtii $5=5 *$ SEXT_ON \\
\hline \multirow[t]{2}{*}{179} & ksxstrtii $4=-5 *$ SEXT_ON \\
\hline & ksxstrtii $3=5 *$ SEXT_ON \\
\hline \multirow[t]{2}{*}{181} & ksxstrtii $2=5 *$ SEXT_ON \\
\hline & ksxstrtii $1=-5 *$ SEXT_ON \\
\hline \multirow[t]{2}{*}{183} & \\
\hline & oD01SXT: DRIFT, $\mathrm{L}=$ \\
\hline \multirow[t]{2}{*}{185} & oD2aSXT: DRIFT, $\mathrm{L}=0.5733333-0.20 ;$ \\
\hline & oD02aSXT: DRIFT, $\mathrm{L}=2.0-0.2 ;$ \\
\hline \multirow[t]{2}{*}{187} & oD03SXT: DRIFT, $\mathrm{L}=0.8-0.2$ \\
\hline & oD1SXT: DRIFT, $\mathrm{L}=3.353333-0.20$ \\
\hline \multirow[t]{2}{*}{189} & oD2SXT: DRIFT, $\mathrm{L}=3.373333-0.20 ;$ \\
\hline & oD0SXT: DRIFT, $\mathrm{L}=0.94-0.20 ;$ \\
\hline \multirow[t]{2}{*}{191} & oD2bSXT: DRIFT, $\mathrm{L}=6.173333-0.20 ;$ \\
\hline & oQSXT: DRIFT, $\mathrm{L}=$ \\
\hline 193 & oASXT: DRIFT, $\mathrm{L}=$ \\
\hline \multirow[t]{2}{*}{195} & \\
\hline & ! LEFT OF SYMMETRRY \\
\hline \multirow[t]{2}{*}{197} & SXIR2IRL1: SEXTUPOLE , $\mathrm{L}=0.2, \mathrm{~K} 2:=\mathrm{KSXIR} 2 \mathrm{IR} 1 ;$ \\
\hline & SXIR2IRL2: SEXTUPOLE , L=0.2 , K2:= KSXIR2IR2; \\
\hline \multirow[t]{2}{*}{199} & SXIR2IRL3: SEXTUPOLE , L=0.2 , K2:= KSXIR2IR3; \\
\hline & SXIR2IRL4: SEXTUPOLE , L=0.2 , K2:= KSXIR2IR4; \\
\hline \multirow[t]{2}{*}{201} & SXIR2IRL5: SEXTUPOLE , L=0.2 , K2:= KSXIR2IR5; \\
\hline & SXIR2IRL6: SEXTUPOLE , L=0.2 , K2:= KSXIR2IR6; \\
\hline 203 & SXIR2IRL7: SEXTUPOLE , L=0.2 , K2:= KSXIR2IR7; \\
\hline \multirow[t]{2}{*}{205} & ! RIGHT OF SYMMETRRY \\
\hline & SXIR2IRR1: SEXTUPOLE , $\mathrm{L}=0.2 \quad, \mathrm{~K} 2:=-\mathrm{KSXIR} 2 \mathrm{IR} 1 ;$ \\
\hline \multirow[t]{2}{*}{207} & SXIR2IRR2: SEXTUPOLE , $\mathrm{L}=0.2 \quad, \mathrm{~K} 2:=-\mathrm{KSXIR} 2 \mathrm{IR} 2 ;$ \\
\hline & SXIR2IRR3: SEXTUPOLE , $\mathrm{L}=0.2 \quad, \mathrm{~K} 2:=-\mathrm{KSXIR} 2 \mathrm{IR} 3$; \\
\hline \multirow[t]{2}{*}{209} & SXIR2IRR4: SEXTUPOLE , $\mathrm{L}=0.2 \quad, \mathrm{~K} 2:=-\mathrm{KSXIR} 2 \mathrm{IR} 4$; \\
\hline & SXIR2IRR5: SEXTUPOLE , $\mathrm{L}=0.2 \quad, \mathrm{~K} 2:=-\mathrm{KSXIR} 2 \mathrm{IR} 5$; \\
\hline \multirow[t]{2}{*}{211} & SXIR2IRR6: SEXTUPOLE , $\mathrm{L}=0.2, \mathrm{~K} 2:=-\mathrm{KSXIR} 2 \mathrm{IR} 6$; \\
\hline & SXIR2IRR7: SEXTUPOLE , $\mathrm{L}=0.2 \quad, \mathrm{~K} 2:=-\mathrm{KSXIR} 2 \mathrm{IR} 7$; \\
\hline 213 & ! Right of 2 nd IIP \\
\hline \multirow[t]{2}{*}{215} & SXIR2ARCR1: SEXTUPOLE , L=0.2, K2:= KSXIR2ARCR1; \\
\hline & SXIR2ARCR2: SEXTUPOLE , L=0.2 , K2:= KSXIR2ARCR2; \\
\hline
\end{tabular}




\begin{tabular}{|c|c|c|}
\hline \multirow[t]{2}{*}{217} & SXIR2ARCR3: SEXTUPOLE , $L=0.2$ & $\mathrm{~K} 2:=\mathrm{KSXIR} 2 \mathrm{ARCR} 3 ;$ \\
\hline & SXIR2ARCR4: SEXTUPOLE , $\mathrm{L}=0.2$ & $\mathrm{~K} 2:=\mathrm{KSXIR} 2 \mathrm{ARCR} 4$ \\
\hline \multirow[t]{2}{*}{219} & SXIR2ARCR5: SEXTUPOLE , $\mathrm{L}=0.2$ & K2:= KSXIR2ARCR5; \\
\hline & SXIR2ARCR6: SEXTUPOLE , $\mathrm{L}=0.2$ & $\mathrm{~K} 2:=\mathrm{KSXIR} 2 \mathrm{ARCR} 6$ \\
\hline \\
\hline \multicolumn{3}{|l|}{23} \\
\hline & ! L of $1 \mathrm{st}$ IIP & \\
\hline \multirow[t]{2}{*}{225} & SXIR2ALCR1: SEXTUPOLE , $\mathrm{L}=0.2$ & $\mathrm{~K} 2:=-\mathrm{KSXIR} 2 \mathrm{ARCR} 1 ;$ \\
\hline & SXIR2ALCR2: SEXTUPOLE , $\mathrm{L}=0.2$ & $\mathrm{~K} 2:=-\mathrm{KSXIR} 2 \mathrm{ARCR} 2 ;$ \\
\hline \multirow[t]{2}{*}{227} & SXIR2ALCR3: SEXTUPOLE , $L=0.2$ & $\mathrm{~K} 2:=-\mathrm{KSXIR} 2 \mathrm{ARCR} 3 ;$ \\
\hline & SXIR2ALCR4: SEXTUPOLE , $\mathrm{L}=0.2$ & $\mathrm{~K} 2:=-\mathrm{KSXIR} 2 \mathrm{ARCR} 4$ \\
\hline \multirow[t]{2}{*}{29} & SXIR2ALCR5: SEXTUPOLE , $\mathrm{L}=0.2$ & $\mathrm{~K} 2:=-\mathrm{KSXIR} 2 \mathrm{ARCR} 5$ \\
\hline & SXIR2ALCR6: SEXTUPOLE , $\mathrm{L}=0.2$ & $\mathrm{~K} 2:=-\mathrm{KSXIR} 2 \mathrm{ARCR} 6 ;$ \\
\hline \multicolumn{3}{|l|}{31} \\
\hline \multirow[t]{2}{*}{233} & SXSTRTII1: SEXTUPOLE , L=0.2 & $\mathrm{K} 2:=\mathrm{KSXST}$ \\
\hline & SXSTRTII2: SEXTUPOLE , $\mathrm{L}=0.2$ & K2:= KSXSTRTII2; \\
\hline \multirow[t]{2}{*}{235} & SXSTRTII3: SEXTUPOLE & K2:= KSXSTRTII3; \\
\hline & SXSTRTII4: SEXTUPOLE , $\mathrm{L}=0.2$ & K2:= KSXSTRTII4; \\
\hline \multirow[t]{2}{*}{237} & SXSTRTII5: SEXTUPOLE , $\mathrm{L}=0.2$ & K2:= KSXSTRTII5; \\
\hline & SXSTRTII6: SEXTUPOLE , L=0.2 & K2:= KSXSTRTII6; \\
\hline \multirow[t]{2}{*}{239} & SXSTRTII7: SEXTUPOLE , L=0.2 & K2:= KSXSTRTII7; \\
\hline & SXSTRTII8: SEXTUPOLE , $\mathrm{L}=0.2$ & K2:= KSXSTRTII8; \\
\hline \multirow[t]{2}{*}{241} & SXSTRTII9: SEXTUPOLE & K2:= KSXSTRTII9; \\
\hline & SXSTRTII10: SEX] & , K2:= KSXSTRTII10; \\
\hline \multirow[t]{2}{*}{243} & SXSTRTII11: SEXTUPOLE , $\mathrm{L}=0.2$ & , K2:= KSXSTRTII11; \\
\hline & SXSTRTII12: SEXTUPOLE , $\mathrm{L}=0.2$ & , K2:= KSXSTRTII12; \\
\hline & SXSTRTII13: SEXTUPOLE , $\mathrm{L}=0.2$ & , K2:= KSXSTRTII13; \\
\hline \multirow[t]{2}{*}{247} & SXSTRTIIL1: SEXTUPOLE , $\mathrm{L}=0.2$ & K2:= -KSXSTRTII1; \\
\hline & SXSTRTIIL2: SEXTUPOLE , $\mathrm{L}=0.2$ & $\mathrm{~K} 2:=-\mathrm{KSXSTRTII} 2$ \\
\hline \multirow[t]{2}{*}{249} & SXSTRTIIL3: SEXTUPOLE , $\mathrm{L}=0.2$ & K2:= -KSXSTRTII3; \\
\hline & SXSTRTIIL4: SEXTUPOLE , $\mathrm{L}=0.2$ & K2:= -KSXSTRTII 4 \\
\hline \multirow[t]{2}{*}{251} & SXSTRTIIL5: SEXTUPOLE , $\mathrm{L}=0.2$ & K2:= -KSXSTRTII5; \\
\hline & SXSTRTIIL6: SEXTUPOLE , $\mathrm{L}=0.2$ & $\mathrm{~K} 2:=-\mathrm{KSXSTRTII} 6$ \\
\hline \multirow[t]{2}{*}{253} & SXSTRTIIL7: SEXTUPOLE , $\mathrm{L}=0.2$ & $\mathrm{~K} 2:=-\mathrm{KSXSTRTII} 7$ \\
\hline & SXSTRTIIL8: SEXTUPOLE , $\mathrm{L}=0.2$ & $\mathrm{~K} 2:=-\mathrm{KSXSTRTII} 8$ \\
\hline \multirow[t]{2}{*}{255} & SXSTRTIIL9: SEXTUPOLE , $\mathrm{L}=0.2$ & K2:= -KSXSTRTII9; \\
\hline & SXSTRTIIL10: SEXTUPOLE, $\mathrm{L}=0$. & $\mathrm{K} 2:=-\mathrm{KSXSTRTII}$ \\
\hline & SXSTRTIIL11: SEXTUPOLE , $\mathrm{L}=0.2$ & $\mathrm{~K} 2:=-\mathrm{KSXSTRTII} 1$ \\
\hline
\end{tabular}









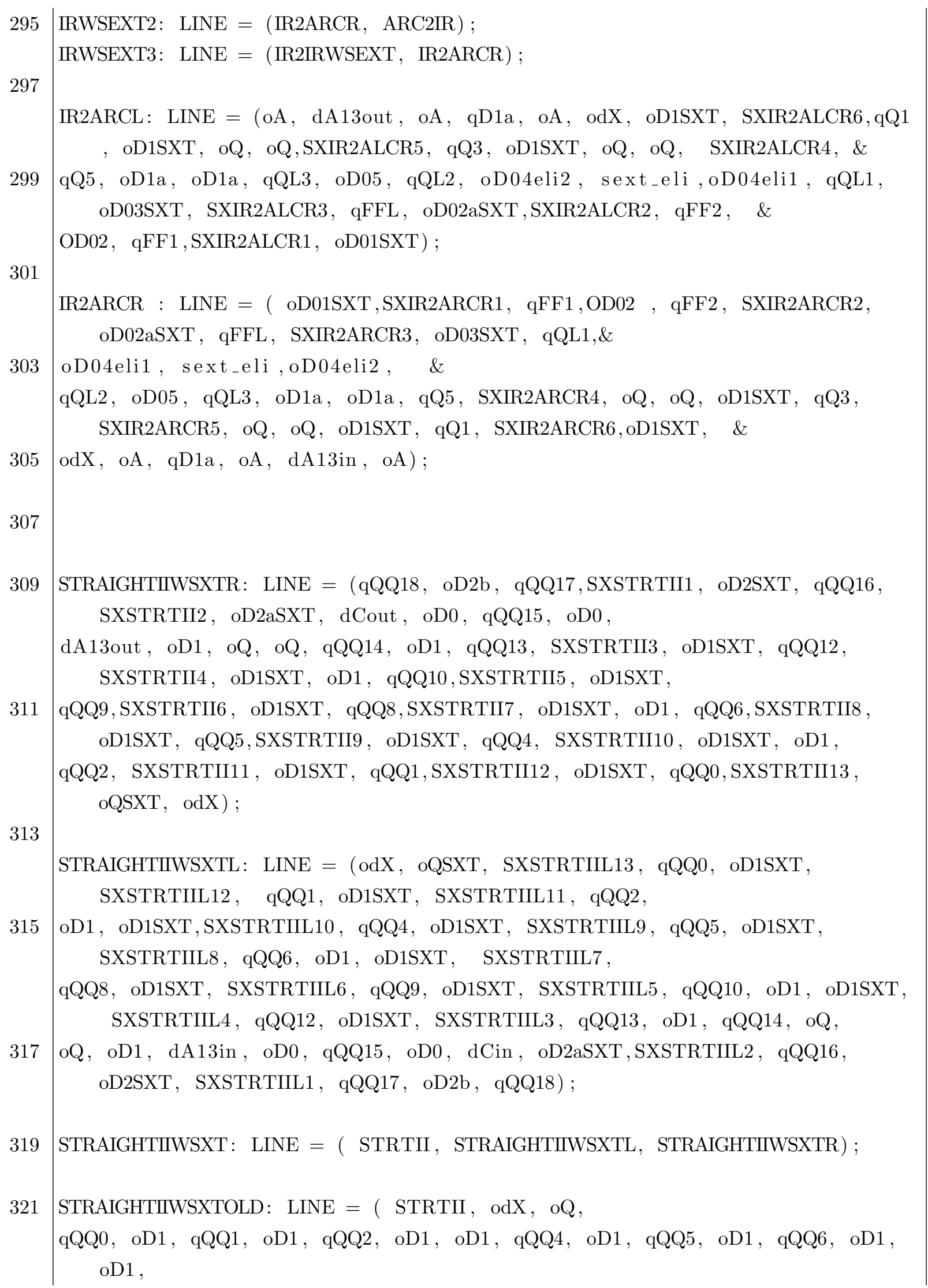


323 qQQ8, oD1, qQQ9, oD1, qQQ10, oD1, oD1, qQQ12, oD1, qQQ13, oD1, qQQ14, $\mathrm{oQ}$,

oQ, oD1, dA13in, oD0, qQQ15, oD0, dCin, oD2a, qQQ16, oD2, qQQ17, oD2b, qQQ18,

qQQ18, oD2b, qQQ17, SXSTRTII1, oD2SXT, qQQ16, SXSTRTII2, oD2aSXT, dCout, oD0, qQQ15, oD0,

dA13out, oD1，oQ，oQ，qQQ14，oD1，qQQ13，SXSTRTII3，oD1SXT, qQQ12, SXSTRTII4, oD1SXT, oD1, qQQ10,SXSTRTII5, oD1SXT, qQQ9, SXSTRTII6 , oD1SXT, qQQ8, SXSTRTII7，oD1SXT，oD1，qQQ6, SXSTRTII8 , oD1SXT, qQQ5, SXSTRTII9, oD1SXT, qQQ4, SXSTRTII10, oD1SXT, oD1, qQQ2， SXSTRTII11，oD1SXT， qQQ1,SXSTRTII12，oD1SXT， qQQ0, SXSTRTII13, oQSXT, odX);

ARCOUTSXTA: LINE $=(\mathrm{qD}$, SXARCOUTA1, oASXT, dAout, oA, $\mathrm{qF}$, SXARCOUTA2, oASXT, dAout, oA);

ARCOUTSXTB: LINE $=(\mathrm{qD}$, SXARCOUTB1, oASXT, dAout , oA , $\mathrm{qF}$, SXARCOUTB2, oASXT, dAout, oA);

ARCOUTSXTC: LINE $=(\mathrm{qD}$, SXARCOUTC1, oASXT, dAout , oA, $\mathrm{qF}$, SXARCOUTC2, oASXT, dAout, oA);

ARCOUTSXTD: LINE $=(\mathrm{qD}$, SXARCOUTD1, oASXT, dAout, oA , qF ,SXARCOUTD2, oASXT, dAout, oA);

ARCINSXTA: LINE $=(\mathrm{dAin}, \mathrm{oA}, \mathrm{qD}$, SXARCINA1, oASXT, dAin, oA, $\mathrm{qF}$, SXARCINA2, OASXT) ;

ARCINSXTB: LINE $=(\mathrm{dAin}, \mathrm{oA}, \mathrm{qD}$, SXARCINB1, oASXT, dAin, oA, $\mathrm{qF}$, SXARCINB2, oASXT) ;

ARCINSXTC: LINE $=(\mathrm{dAin}, \mathrm{oA}, \mathrm{qD}$, SXARCINC1, oASXT, dAin, oA, $\mathrm{qF}$, SXARCINC2, oASXT) ;

ARCINSXTD: LINE $=(\mathrm{dAin}, \mathrm{oA}, \mathrm{qD}$, SXARCIND1, oASXT, dAin, oA, $\mathrm{qF}$, SXARCIND2, oASXT) ;

ARCOUTSXT: LINE = (ARCOUTSXTA, ARCOUTSXTB, ARCOUTSXTC, ARCOUTSXTD) ;

ARCINSXT: LINE $=($ ARCINSXTA, ARCINSXTB, ARCINSXTC, ARCINSXTD $)$;

IR2IR： LINE=(IIR1，oD01， qFF1，oD02， qFF2，oD02a， qFFR，oD03， qQR1, 
349 oD04, qQR2, oD05, qQ6, oD1, dA13out, oD0, qQ13, oD0, dCout, oD2, qQ14, $\mathrm{oD} 2$, qQ15，oD2， qQ16，qQ16，oD2， qQ15，oD2，qQ14，oD2， dCin， oD0, qQ13，oD0,

dA13in, oD1, qQ6, oD05, qQR2, oD04, qQR1, oD03, qFFR, oD02a, qFF2, oD02

IR $:$ LINE $=($ ARC2IR, oA, dA13out, oA, qD1a, oA, odX, oD1, qQ1, oD1, oQ, oQ, qQ3, oD1, oQ, oQ,

355 qQ5, oD1a, oD1a, qQL3, oD05, qQL2, oD04, qQL1, oD03, qFFL, oD02a, qFF2, oD02, qFF1, oD01, IR2IR, oD01, qFF1, oD02, qFF2, oD02a, qFFL, oD03, qQL1, oD04, qQL2, oD05, qQL3, oD1a, oD1a，qQ5，oD1，oQ，oQ，qQ3，oD1，oQ，oQ， qQ1, $\mathrm{oD} 1$ odX, oA, qD1a, oA, dA13in, oA, ARC2IR);

STRAIGHTII : LINE $=($ STRTII, odX, oQ,

361 qQQ0, oD1, qQQ1, oD1, qQQ2, oD1, oD1, qQQ4, oD1, qQQ5, oD1, qQQ6, oD1, oD1

qQQ8，oD1， qQQ9，oD1，qQQ10，oD1，oD1，qQQ12，oD1， qQQ13，oD1， qQQ14， $\mathrm{oQ}$

oQ, oD1, dA13in, oD0, qQQ15, oD0, dCin, oD2a, qQQ16, oD2, qQQ17, oD2b, qQQ18,

qQQ18, oD2b, qQQ17, oD2, qQQ16, oD2a, dCout, oD0, qQQ15, oD0,

dA13out, oD1，oQ，oQ，qQQ14，oD1，qQQ13，oD1，qQQ12，oD1，oD1， qQQ10, oD1,

qQQ9, oD1，qQQ8，oD1，oD1，qQQ6，oD1， qQQ5，oD1，qQQ4，oD1，oD1， qQQ2, oD1

ARCOUT: $\mathrm{LINE}=(\mathrm{qD}, \mathrm{oA}$, dAout, oA, $\mathrm{qF}, \mathrm{oA}, \mathrm{dAout}, \mathrm{oA})$;

ARCIN $:$ LINE $=($ dAin, oA, $q$ D, oA, dAin, oA, $q F, o A)$;

373 MACHINE: LINE $=(\mathrm{oA}, \mathrm{qD} 1 \mathrm{~b}, \mathrm{oA}, \mathrm{dA} 13 \mathrm{out}, \mathrm{oA}, \mathrm{qF} 1 \mathrm{~b}, \mathrm{oA}, \mathrm{dA23out,}$ oA, qD2, oA, dA23out, oA, qF2, oA, dAout, oA, qD3, oA, dAout, oA, qF3, oA, dAout, oA 


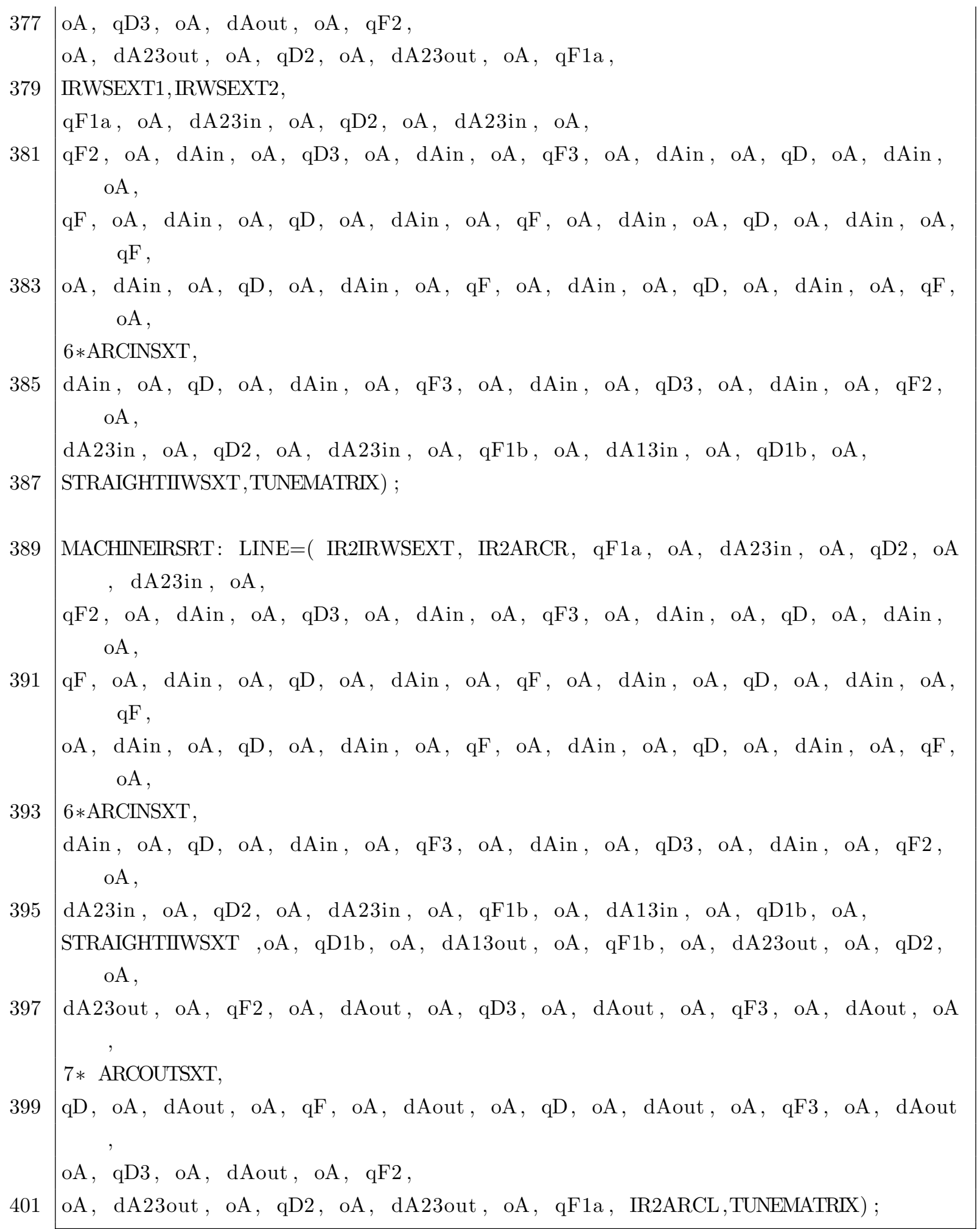




\section{VITA}

Hisham Kamal Sayed

Department of Physics

Old Dominion University

Norfolk, VA 23529

\section{EDUCATION}

B.Sc. Physics and Astronomy May 2001 Cairo University, Giza, Egypt.

M.Sc. Physics May 2007 Old Dominion University, Norfolk, VA USA.

\section{HONORS and AWARDS}

Teaching Accelerator Physics United States Particle Accelerator School, Old Domnion University January 2011.

S.I.A.M award for problem solving competition March 2009.

Research visiting fellow Cornell University, Ithaca NY 2008-2009.

Research assistantship Thomas Jefferson National Accelerator Facility 20072011.

\section{PUBLICATIONS}

Spin rotator optics for MEIC H. Sayed et al., International Particle Accelerator Conference, IPAC 2010.

Correction of the chromaticity up to second order for MEIC H. Sayed et al., IPAC 2010

Initial beam results from the Cornell high-current ERL Injector Prototype I. Bazarov et al., PAC, 23rd Particle Accelerator Conference, May 2009.

Thermal emittance and response time measurements of a GaN photocathode I. Bazarov et al., Journal of Applied Physics 105 (2009) 083715.

Typeset using $\mathrm{AT}_{\mathrm{E} X}$. 